\title{
Symmetric Gapped Interfaces of SPT and SET States: Systematic Constructions
}

\author{
Juven Wang, ${ }^{1}$ Xiao-Gang Wen, ${ }^{2}$ and Edward Witten ${ }^{1}$ \\ ${ }^{1}$ School of Natural Sciences, Institute for Advanced Study, Princeton, New Jersey 08540, USA \\ ${ }^{2}$ Department of Physics, Massachusetts Institute of Technology, Cambridge, Massachusetts 02139, USA
}

(Received 29 August 2017; revised manuscript received 1 April 2018; published 22 August 2018)

Symmetry-protected topological (SPT) states have boundary 't Hooft anomalies that obstruct the effective boundary theory realized in its own dimension with UV completion and with an on-site $G$-symmetry. In this work, yet we show that a certain anomalous non-on-site $G$-symmetry along the boundary becomes on-site when viewed as an extended $H$-symmetry, via a suitable group extension $1 \rightarrow K \rightarrow H \rightarrow G \rightarrow 1$. Namely, a nonperturbative global (gauge or gravitational) anomaly in $G$ becomes anomaly free in $H$. This guides us to construct an exactly soluble lattice path integral and Hamiltonian of symmetric gapped boundaries applicable to any SPT state of any finite symmetry group, including on-site unitary and antiunitary time-reversal symmetries. The resulting symmetric gapped boundary can be described either by an $H$-symmetry extended boundary in any spacetime dimension or, more naturally, by a topological emergent $K$-gauge theory with a global symmetry $G$ on a $3+1 \mathrm{D}$ bulk or above. The excitations on such a symmetric topologically ordered boundary can carry fractional quantum numbers of the symmetry $G$, described by representations of $H$. (Applying our approach to a $1+1 \mathrm{D}$ boundary of $2+1 \mathrm{D}$ bulk, we find that a deconfined gauge boundary indeed has spontaneous symmetry breaking with long-range order. The deconfined symmetry-breaking phase crosses over smoothly to a confined phase without a phase transition.) In contrast to known gapped boundaries or interfaces obtained via symmetry breaking (either global symmetry breaking or the Anderson-Higgs mechanism for gauge theory), our approach is based on symmetry extension. More generally, applying our approach to SPT states, topologically ordered gauge theories, and symmetry enriched topologically ordered (SET) states leads to generic boundaries or interfaces constructed with a mixture of symmetry breaking, symmetry extension, and dynamical gauging.

DOI: 10.1103/PhysRevX.8.031048

Subject Areas: Condensed Matter Physics,

Particles and Fields,

Strongly Correlated Materials

\section{INTRODUCTION}

After the realization that a spin-1/2 antiferromagnetic Heisenberg chain in $1+1$ dimensions $(1+1 D)$ admits a gapless state $[1,2]$ that "nearly" breaks the spin rotation symmetry (i.e., it has "symmetry-breaking" spin correlation functions that decay algebraically), many physicists expected that spin chains with higher spin, having fewer quantum fluctuations, might also be gapless with algebraic long-range spin order. However, Haldane [3] first realized that antiferromagnetic Heisenberg spin chains in 1+1D with integer spins have a gapped disordered phase with short-range spin correlations. At first, it was thought that those states are trivial disordered states, like a product state

Published by the American Physical Society under the terms of the Creative Commons Attribution 4.0 International license. Further distribution of this work must maintain attribution to the author(s) and the published article's title, journal citation, and DOI. of spin-0 objects. Later, it was discovered that they can have degenerate zero-energy modes at the ends of the chain [4], similar to the gapless edge states of quantum Hall systems. This discovery led to a suspicion that these gapped phases of antiferromagnetic integer spin chains might be topological phases.

Are Haldane phases topological or not topological? What kind of "topological" is it? That was the question. It turns out that only odd-integer-spin Haldane phases (each site with an odd-integer spin) are topological, while the even-integer-spin Haldane phases (each site with an eveninteger spin) are really trivial (a trivial vacuum ground state like the product state formed by spin-0's). The essence of nontrivial odd-integer-spin Haldane phases was obtained in Ref. [5], based on a tensor network renormalization calculation [6], where simple fixed-point tensors characterizing quantum phases can be formulated. It was discovered that the spin-1 Haldane phase is characterized by a nontrivial fixed-point tensor-a corner-double-line tensor. The corner-double-line structure implies that the spin-1 Haldane 
phase is actually equivalent to a product state, once we remove its global symmetry. However, Ref. [5] showed that the corner-double-line tensor is robust against any local perturbations that preserve certain symmetries [namely, $S O(3)$ symmetry in the case of the integer spin chain], but it flows to the trivial fixed point tensor if we break the symmetry. This suggests that, in the presence of symmetry, even a simple product state can be nontrivial (i.e., distinct from the product state of spin-0's that has no cornerdouble-line structure), and such nontrivial symmetric product states were named symmetry-protected topological states (SPTs). (Despite its name, a SPT state has no intrinsic topological order in the sense defined in Refs. [7,8]. By this definition, a SPT state with no topological order cannot be deformed into a trivial disordered gapped phase in a symmetry-preserving fashion.)

Since SPT states are equivalent to simple product states if we remove their global symmetry, one quickly obtains their classification in 1+1D [9-11], in terms of projective representations [12] of the symmetry group $G$. As remarked above, one found that only the odd-integer-spin Haldane phases are nontrivial SPT states. The even-integer-spin Haldane phases are trivial gapped states, just like the disordered product state of spin-0's [13]. Soon after their classification in $1+1 \mathrm{D}$, bosonic SPT states in higher dimensions were also classified based on group cohomology $\mathcal{H}^{d+1}[G, U(1)]$ and $\mathcal{H}^{d+1}[G \times S O(\infty), U(1)]$ [14-18] or based on cobordism theory [19-21]. In fact, SPT states and Dijkgraaf-Witten gauge theories [22] are closely related: Dynamically gauging the global symmetry $[23,24]$ in a SPT state leads to a corresponding Dijkgraaf-Witten gauge theory.

To summarize, SPT states are the simplest of symmetric phases and, accordingly, have another name: symmetryprotected trivial states. They are quantum-disordered product states that do not break the symmetry of the Hamiltonian. Naively, one would expect that such disordered product states all have nonfractionalized bulk excitations. What is nontrivial about a SPT state is more apparent if one considers its possible boundaries. For any bulk gapped theory with $G$ symmetry, a $G$-preserving boundary is described by some effective boundary theory with symmetry $G$. However, the boundary theories of different SPT states have different anomalies in the global symmetry $G$ [25-28]. A simple explanation follows: While the bulk of a SPT state of a symmetry group $G$ has an on-site symmetry, the boundary theory of SPT state has an effective non-on-site $G$-symmetry. Non-on-site $G$-symmetry means that the $G$-symmetry does not act in terms of a tensor product structure on each site, namely, the $G$-symmetry acts nonlocally on several effective boundary sites. Non-on-site symmetry cannot be dynamically gauged - because conventionally the gauging process requires inserting gauge variables on the links between the local site variables of $G$-symmetry. Thus, the boundary of a SPT state of a symmetry $G$ has an obstruction to gauging, as 't Hooft anomaly obstruction to gauging a global symmetry [29]. Such an anomalous boundary is the essence of a SPT state: Different boundary anomalies characterize different bulk SPT states. In fact, different SPT states classify gauge and mixed gauge-gravity anomalies in one lower dimension [26-28,30,34].

From the above discussion, we realize that to understand the physical properties of SPT states is to understand the physical consequence of anomalies in the global symmetry $G$ on the boundary of SPT states. For a $1+1 \mathrm{D}$ boundary, it was shown that the anomalous global symmetry makes the boundary gapless and/or symmetry breaking [14]. However, in higher dimensions, there is a third possibility: The boundary can be gapped, symmetry preserving, and topologically ordered. (This third option is absent for a $1+1 \mathrm{D}$ boundary roughly because there is no bosonic topological order in that dimension [37].) Concrete examples of topologically ordered symmetric boundaries have been constructed in particular cases [39-47]. In this paper, we give a systematic construction that applies to any SPT state with any finite [48] symmetry group $G$, for any boundary of dimension $2+1$ or more. Namely, we show that symmetrypreserving gapped boundary states always exist for any $d+1 \mathrm{D}$ bosonic SPT state with a finite symmetry group $G$ when $d \geq 3$. We also study a few examples, but less systematically, when SPT states have continuous compact Lie groups $G$, and we study their symmetry-preserving gapped boundaries, which may or may not exist.

Symmetry breaking gives a straightforward way to construct gapped boundary states or interfaces, since SPT phases are completely trivial if one ignores the symmetry. For topological phases described by group cocycles of a group $G$, the symmetry-breaking mechanism can be described as follows. It is based on breaking the $G$ to a subgroup $G^{\prime} \subseteq G$, corresponding to an injective homomorphism $t$ as

$$
G^{\prime} \stackrel{\iota}{\rightarrow} G
$$

Here, $G^{\prime}$ must be such that the cohomology class in $\mathcal{H}^{d+1}[G, U(1)]$ that characterizes the $d+1 \mathrm{D}$ SPT or symmetry enriched topologically ordered states (SETs) becomes trivial when pulled back (or equivalently restricted) to $G^{\prime}$. The statement that the class is "trivial" does not mean that the relevant $G$ cocycle is 1 if we restrict its argument from $G$ to $G^{\prime}$, but that this cocycle becomes a coboundary when restricted to $G^{\prime}$.

Our approach to constructing exactly soluble gapped boundaries does not involve symmetry breaking, but what one might call "symmetry extension":

$$
1 \rightarrow K \rightarrow H \stackrel{r}{\rightarrow} G \rightarrow 1 .
$$

Here, we extend $G$ to a larger group $H$, such that $G$ is its quotient group, $K$ is its normal subgroup, and $r$ is a surjective group homomorphism, more or less opposite to 
the injective homomorphism $\imath$ related to symmetry breaking [Eq. (1.1)]. $H$ and $r$ must be such that the cohomology class in $\mathcal{H}^{d+1}[G, U(1)]$ that characterizes the SPT or SET state becomes trivial when pulled back to $H$. For any finite $G$ and any class in $\mathcal{H}^{d+1}[G, U(1)]$, we show that suitable choices of $H$ and $r$ always exist, when the bulk space dimension $d \geq 1$. Physically, the gapped phases that we construct in this way have the property that boundary degrees of freedom transform under an $H$-symmetry. However, in condensed matter applications, one should usually [49] assume that the subgroup $K$ of $H$ is gauged, and then (in the SPT case) the global symmetry acting on the boundary is $G$, just as in the bulk. So, in that sense, when all is said and done, the boundary states that we construct simply have the same global symmetry as the bulk, and the boundaries become topological since $K$ is gauged. For $2+1 \mathrm{D}$ (or higher dimensional) boundaries, such symmetry-preserving topological boundaries may have excitations with fractional $G$-symmetry quantum numbers. The fact that the boundary degrees of freedom are in representations of $H$ rather than $G$ actually describes such a charge fractionalization.

The idea behind this work was described in a somewhat abstract way in Sec. 3.3 of Ref. [51], and a similar idea was used in Ref. [52] in examples. In the present paper, we develop this idea in detail and in a down-to-earth way, with both spatial lattice Hamiltonians and spacetime lattice path integrals that are ultraviolet (UV) complete at the lattice high-energy scale. We also construct a mixture combining the symmetry-breaking and symmetry-extension mechanisms.

We further expand our approach to construct anomalous gapped symmetry-preserving interfaces (i.e., domain walls) between bulk SPT states, topological orders (TO), and SETs [53]. We will recap the terminology for the benefit of some readers. SPTs are short-range entangled (SRE) states, which can be deformed to a trivial product state under local unitary transformations at the cost of breaking some protected global symmetry. Examples of SPTs include topological insulators [54-56]. Topological orders are long-range entangled (LRE) states, which cannot be deformed to a trivial product state under local unitarity transformations even if breaking all global symmetries. SETs are topological orders-thus, LRE states-but additionally have some global symmetry. Being long-range entangled, TOs and SETs have richer physics and mathematical structures than the short-range entangled SPTs. Examples of TOs and SETs include fractional quantum Hall states and quantum spin liquids [57]. In this work, for TOs and SETs, we mainly focus on those that can be described by Dijkgraaf-Witten twisted gauge theories, possibly extended with global symmetries. We comment on possible applications and generalizations to gapped interfaces of bosonic or fermionic topological states obtained from beyond-group cohomology and cobordism theories in Secs. VI and VII.

\section{A MODEL THAT REALIZES THE $2+1 \mathrm{D} Z_{2}$ SPT STATE: CZX MODEL}

The first lattice model that realizes a $2+1 \mathrm{D}$ SPT state (the $Z_{2}$-SPT state) was introduced by Chen et al. [14] and was named the CZX model. The CZX model is a model on a square lattice (Fig. 1), where each lattice site contains four qubits, or objects of spin $1 / 2$. For each spin, we use a basis $|\uparrow\rangle$ and $|\downarrow\rangle$ of $\sigma^{z}$ eigenstates. Thus, a single site has a Hilbert space of dimension $2^{4}$.

Now, let us introduce a $Z_{2}$-symmetry transformation. An obvious choice is the operator that acts on each site $s$ as

$$
U_{X, s}=\prod_{j=1}^{4} \sigma_{j}^{x}, \quad U_{X, s}^{2}=1,
$$

which simply flips the four spins in site $s$. However, to construct the CZX model, a more subtle choice is made. In this model, in the basis $|\uparrow\rangle,|\downarrow\rangle$, the flip operator $U_{X, s}$ is modified with \pm signs. For a pair of spins $i, j$, we define an operator [58] $U_{C Z, i j}$ that acts as -1 if spins $i, j$ are both in state $|\downarrow\rangle$ and otherwise acts as +1 . There are various ways to describe $U_{C Z, i j}$ by a formula:

$$
\begin{aligned}
U_{C Z, i j} & =\frac{1+\sigma_{i}^{z}+\sigma_{j}^{z}-\sigma_{i}^{z} \sigma_{j}^{z}}{2} \\
& =\mathrm{i}^{\left(\sigma_{i}^{z}+\sigma_{j}^{z}-\sigma_{i}^{z} \sigma_{j}^{z}-1\right) / 2} .
\end{aligned}
$$

Now, for a site $s$ that contains four spins $j=1, \ldots, 4$ in cyclic order, we define

$$
U_{C Z, s}=\prod_{j=1}^{4} U_{C Z, j j+1} .
$$

The $Z_{2}^{\prime}$-symmetry of the spins at site $s$ is defined as

$$
U_{\mathrm{CZX}, s}=U_{X, s} U_{C Z, s} .
$$

By a short exercise, one can verify that $U_{X, s}$ and $U_{C Z, s}$ commute and, accordingly, that $U_{\mathrm{CZX}, s}^{2}=1$. The $Z_{2}$-symmetry

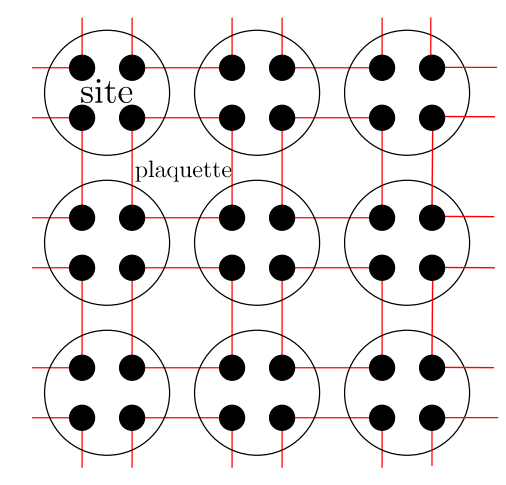

FIG. 1. The CZX model. Each site (a large disc) contains four qubits or objects of spin $1 / 2$ (shown as small black dots). The squares, formed by red links, are plaquettes, introduced later. 
generator of the CZX model is defined as a product over all sites of $U_{\mathrm{CZX}, s}$ :

$$
U_{\mathrm{CZX}}=\prod_{s} U_{\mathrm{CZX}, s} .
$$

Clearly, this is an on-site symmetry, that is, it acts separately on the Hilbert space associated to each site. Being on-site, the symmetry is gaugeable and anomaly free. We have not yet picked a Hamiltonian for the CZX model, but whatever $U_{\mathrm{CZX}}$-invariant Hamiltonian we pick, the $Z_{2^{-}}$ symmetry can be gauged by coupling to a $Z_{2}$ lattice gauge field that will live on links that connect neighboring sites.

What we have done so far is trivial in the sense that, by a change of basis on each site, we could have put $U_{\mathrm{CZX}, s}$ in a more standard form. However, this would complicate the description of the Hamiltonian and ground-state wave function of the CZX model, which we come to next.

It is easier to first describe the desired ground-state wave function of the model and then describe a Hamiltonian that has that ground state. In Fig. 1, we have drawn squares that contain four spins, one from each of four neighboring sites. We call these squares "plaquettes." For each plaquette $p$, we define the wave function $\left|\Psi_{p}\right\rangle=(1 / \sqrt{2})(|\uparrow \uparrow \uparrow \uparrow\rangle+|\downarrow \downarrow \downarrow \downarrow\rangle)$. The ground state of the CZX model in the bulk is given by a product over all plaquettes of this wave function for each plaquette:

$$
\left|\Psi_{\mathrm{gs}}\right\rangle=\prod_{p}\left|\Psi_{p}\right\rangle=\prod_{p} \frac{1}{\sqrt{2}}(|\uparrow \uparrow \uparrow \uparrow\rangle+|\downarrow \downarrow \downarrow \downarrow\rangle) .
$$

This state is $U_{\mathrm{CZX}}$ invariant,

$$
U_{\mathrm{CZX}}\left|\Psi_{\mathrm{gs}}\right\rangle=\left|\Psi_{\mathrm{gs}}\right\rangle,
$$

if we define the whole system on a torus without boundary (i.e., with periodic boundary conditions). But that fact is not completely trivial: It depends on cancellations among $C Z_{i j}$ factors for adjacent pairs of spins; see Fig. 2.

Clearly, the entanglement in this wave function is short range, and this wave function describes a gapped state. Moreover, if we would regard the plaquettes (rather than the large discs in Fig. 1) as "sites," then this wave function would be a trivial product state. But in that case, the $Z_{2}$ symmetry of the model would not be on-site. The subtlety of the model comes from the fact that we cannot simultaneously view it as a model with on-site symmetry and a model with a trivial product ground state.

The most obvious Hamiltonian with $\left|\Psi_{\mathrm{gs}}\right\rangle$ as its ground state would be a sum over all plaquettes $p$ of an operator $H_{p}^{0}$ that flips all spins in plaquette $p$ :

$$
\begin{aligned}
& H^{0}=\sum_{p} H_{p}^{0}, \\
& H_{p}^{0}=-(|\uparrow \uparrow \uparrow \uparrow\rangle\langle\downarrow \downarrow \downarrow \downarrow|+| \downarrow \downarrow \downarrow \downarrow\rangle\langle\uparrow \uparrow \uparrow \uparrow \uparrow|) .
\end{aligned}
$$

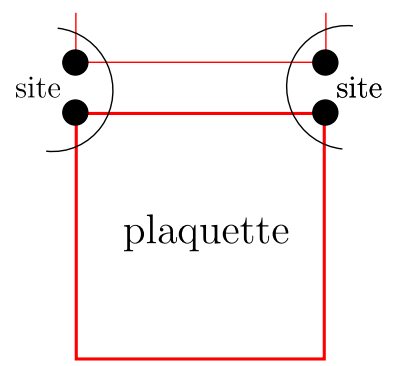

FIG. 2. A pair of adjacent spins: To preserve the symmetry $U_{\mathrm{CZX}}$, we choose a Hamiltonian that only flips the spins in a plaquette if pairs of adjacent spins in neighboring plaquettes are equal. Thus, the spins shown here at the top of this plaquette are only flipped if the two spins just above them are equal. Both the spins in the plaquette and the ones just above them are in different sites, as shown.

This Hamiltonian commutes with the obvious $Z_{2^{-}}$ symmetry that flips all the spins, but does not commute with the more subtle symmetry $U_{\mathrm{CZX}}$. To commute with $U_{\mathrm{CZX}}$, we modify $H^{0}$ to only flip the spins in a plaquette if adjacent pairs of spins in the neighboring plaquettes are equal (Fig. 2). For a plaquette $p$, we define operators $P_{p}^{\alpha} \equiv$ $|\uparrow \uparrow\rangle\langle\uparrow \uparrow|+| \downarrow \downarrow\rangle\langle\downarrow \downarrow|$ that project onto states in which the two spins adjacent to $p$ in the $\alpha$ direction (where $\alpha$ equals up, down, left, or right, denoted as $u, d, l$, or $r$ ) are equal. Then, the CZX Hamiltonian is defined to be

$$
\begin{aligned}
H & =\sum_{p} H_{p} \\
H_{p} & =-(|\uparrow \uparrow \uparrow \uparrow\rangle\langle\downarrow \downarrow \downarrow \downarrow|+| \downarrow \downarrow \downarrow \downarrow\rangle\langle\uparrow \uparrow \uparrow \uparrow|) \otimes_{\alpha} P_{p}^{\alpha} .
\end{aligned}
$$

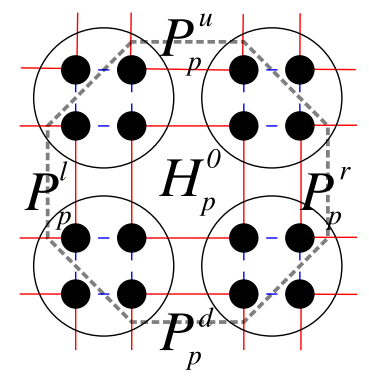

(a)

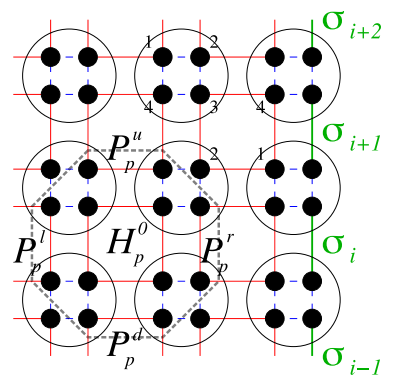

(b)
FIG. 3. Each plaquette Hamiltonian $H_{p}$ acts on the spins contained in an octagon, as depicted in a dashed gray line in the left subfigure (a) and also in the lower left of the right subfigure (b). In the subfigure (b), the octagon in the lower left contains the four spins in plaquette $p$ and four adjacent pairs of spins. In the case of a finite sample made of complete sites, as depicted here, most of the spins can be grouped in plaquettes, but there is a row of spins on the boundary-shown here on the right of the figure - that are not contained in any plaquette. However, the Hamiltonian acts on these boundary spins through the projection operators $P_{p}^{\alpha}$ from a neighboring plaquette. 
Thus, each $H_{p}$ acts on the spins contained in an octagon [Fig. 3], flipping the spins in a plaquette if all adjacent pairs of spins are equal. This Hamiltonian is $U_{\mathrm{CZX}}$ invariant,

$$
\left[U_{\mathrm{CZX}}, H\right]=0,
$$

in the case of a system without boundary (an infinite system or a finite system with periodic boundary conditions). The state $\left|\Psi_{\mathrm{gs}}\right\rangle$ is a symmetry-preserving ground state with shortrange entanglement. However, it is a nontrivial symmetryprotected topological or SPT state. This becomes clear if we examine possible boundaries of the CZX model.

\section{BOUNDARIES OF THE CZX MODEL}

\section{A. The first boundary of the CZX model: 1+1D symmetry-preserving gapless boundary with a non-on-site global $Z_{2}$-symmetry}

The boundary of the CZX model that was studied in the original paper is a very natural one, in which one simply considers a finite system with an integer number of sites [Fig. 3]. One groups the spins into plaquettes, as before, but as shown in the figure, there is a row of spins on the boundary that are not contained in any complete plaquette. We call these the boundary spins.

We define the Hamiltonian as in Eq. (2.9), where now the sum runs over complete plaquettes only. Because the boundary spins are not contained in any complete plaquette, the system is no longer gapped. However, the boundary spins are not completely free to fluctuate at no cost in energy. The reason is that, to minimize the energy, a pair of boundary spins that are adjacent to a plaquette $p$ are constrained to be equal. This is because of the projection operators $P_{p}^{\alpha}$ in the definition of $H_{p}$.

Hence, in a state of minimum energy, the boundary spins are locked together in pairs. These pairs are denoted as $\sigma_{i}$, $\sigma_{i+1}$, etc., in Fig. 3(b), and one can think of them as composite spins.

How does the $Z_{2}$-symmetry generated by $U_{\mathrm{CZX}}$ act on the composite spins? Evidently, $U_{\mathrm{CZX}}$ will flip each composite spin. However, $U_{\mathrm{CZX}}$ also acts by a $C Z$ operation on each adjacent pair of composite spins $\sigma_{i}, \sigma_{i+1}$. That is because, for example, in Fig. 3, the "upper" spin making up the composite spin $\sigma_{i}$ and the "lower" spin making up $\sigma_{i+1}$ are adjacent spins contained in the same site $s$ in the underlying square lattice. Accordingly, in the $Z_{2}$ generator $U_{\mathrm{CZX}, s}$ for site $s$, there is a $C Z$ factor linking these two spins.

Therefore, the effective $Z_{2}$ generator for the composite spins on the boundary is

$$
\hat{U}_{Z_{2}}=\prod_{i} \sigma_{i}^{x} U_{C Z, i i+1}
$$

The product runs over all composite spins $\sigma_{i} ; \hat{U}_{Z_{2}}$ is the product of operators $\sigma_{i}^{x}$ that flip $\sigma_{i}$ and operators $U_{C Z, i i+1}$ that give the usual $C Z$ sign factors for each successive pair of composite spins. Clearly, this effective $Z_{2}$-symmetry is not on-site. No matter how we group a finite set of composite spins into boundary sites, the operator $U_{Z_{2}}$ will always contain $C Z$ factors linking one site to the next [59].

With the Hamiltonian as we have described it so far, all states labeled by any values of the composite spins $\sigma_{i}$, but with complete bulk plaquettes placed in their ground state $\left|\Psi_{p}\right\rangle$, are degenerate. Of course, it is possible to add perturbations that partly lift the degeneracy. However, it has been shown in Ref. [14] that the non-on-site nature of the effective $Z_{2}$-symmetry gives an obstruction to making the boundary gapped and symmetry preserving.

\section{B. The second boundary of the CZX model: 1 + 1D gapped boundary by extending the $Z_{2}$-symmetry to a $Z_{4}$-symmetry}

The main idea of the present paper can be illustrated by a simple alternative boundary of the CZX model. To construct this boundary, we simply omit the boundary spins from the previous discussion. This means that, now, the system is made of complete plaquettes, even along the boundary (Fig. 4), but there is a row of boundary spins that are not in complete sites. As indicated in the figure, we combine the boundary spins in pairs into boundary sites. Thus, a boundary site has only two spins, while a bulk site has four. In the figure, we have denoted the "upper" and "lower" spins in the $i$ th boundary site as $\sigma_{i+}$ and $\sigma_{i-}$.

To specify the model, we should specify what the Hamiltonian looks like near the boundary and how the global symmetry is defined for the boundary spins. First of all, now that all spins are in complete plaquettes, we can look for a gapped system with the same ground-state wave function as in Eq. (2.6):

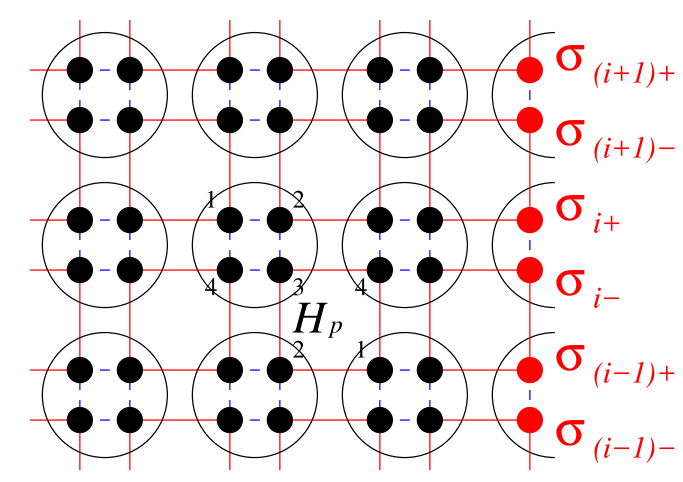

FIG. 4. By omitting the right row of spins from the boundary of Fig. 3(b), we get an alternative boundary of the CZX model. Now all spins are contained in plaquettes, but on the boundary there are "incomplete sites," shown as semicircles on the right of the figure, that contain only two spins instead of four. The "upper" and "lower" spins of the $i$ th boundary site have been labeled $\sigma_{i+}$ and $\sigma_{i-\text {. }}$. 


$$
\left|\Psi_{\mathrm{gs}}\right\rangle=\prod_{p}\left|\Psi_{p}\right\rangle=\prod_{p} \frac{1}{\sqrt{2}}(|\uparrow \uparrow \uparrow \uparrow\rangle+|\downarrow \downarrow \downarrow \downarrow\rangle) .
$$

To get this ground state, we define the Hamiltonian by the same formula as in Eq. (2.9). Only one very small change is required: A boundary plaquette is adjacent to only three pairs of spins instead of four, so in the definition of $H_{p}$ in Eq. (2.9), if $p$ is a boundary plaquette, the product of projection operators $\otimes_{\alpha} P_{p}^{\alpha}$ contains only three factors and not four.

The last step is to define the action of the global " $Z_{2}$ " symmetry for boundary sites. We have put " $Z_{2}$ " in quotes for a reason that will be clear in a moment. Once we have chosen the Hamiltonian as above, the choice of the global symmetry generator is forced on us. The symmetry generator at the $i$ th boundary site will have to flip the two spins $\sigma_{i+}$ and $\sigma_{i-}$, of course, but it also needs to have a $C Z$ factor linking these two spins. So, the symmetry generator of the $i$ th boundary site will have to be

$$
U_{\mathrm{CZX}, i}=\sigma_{i+}^{x} \sigma_{i-}^{x} U_{C Z, i+i-} .
$$

The full symmetry generator is

$$
U_{\mathrm{CZX}}=\prod_{s} U_{\mathrm{CZX}, s}
$$

where the product runs over all bulk or boundary sites $s$, and $U_{\mathrm{CZX}, s}$ is defined in the usual way for bulk sites and as in Eq. (3.3) for boundary states.

We have found a gapped, symmetry-preserving boundary state for the CZX model. There is a catch, however. The global symmetry is no longer $Z_{2}$. Although the operator $U_{\mathrm{CZX}, s}$ squares to 1 if $s$ is a bulk site, this is not so for boundary sites. Rather, from Eq. (3.3), we find that, for a boundary site,

$$
U_{\mathrm{CZX}, i}^{2}=-\sigma_{i+}^{z} \sigma_{i-}^{z} .
$$

This operator is -1 if the two spins $\sigma_{i+}$ and $\sigma_{i-}$ in the $i$ th boundary site are both up or both down, and otherwise +1 . Clearly, $U_{\mathrm{CZX}, i}^{2} \neq 1$, so the full global symmetry generator $U_{\mathrm{CZX}}$ does not obey $U_{\mathrm{CZX}}^{2}=1$ but rather

$$
U_{\mathrm{CZX}}^{4}=1 .
$$

Thus, rather than the symmetry being broken by our choice of boundary state, it has been enhanced from $Z_{2}$ to $Z_{4}$. But a $Z_{2}$ subgroup of $Z_{4}$ generated by $U_{\mathrm{CZX}}^{2}$ acts only on the boundary, since $U_{\mathrm{CZX}}^{2}=1$ for bulk sites.

What we have here is a group extension,

$$
1 \rightarrow K \rightarrow H \rightarrow G \rightarrow 1 .
$$

$G=Z_{2} \equiv Z_{2}^{G}$ is the global symmetry group of the bulk theory, $H=Z_{4} \equiv Z_{4}^{H}$ is the global symmetry of the complete system including its boundary, and $K=Z_{2} \equiv Z_{2}^{K}$ (or a different $Z_{2}^{\prime}$ ) is the subgroup of $H$ that acts only along the boundary. In this case, we denote the exact sequence Eq. (3.7) also as

$$
0 \rightarrow Z_{2}^{K} \rightarrow Z_{4}^{H} \rightarrow Z_{2}^{G} \rightarrow 0 .
$$

As was explained from an abstract point of view in Sec. 3.3 of Ref. [51], and as we will explain more concretely later in this paper, when certain conditions are satisfied, such a group extension along the boundary gives a way to construct gapped boundary states of a bulk SPT phase. (As we explain in detail later, the relevant condition is that the cohomology class of $G$ that characterizes the SPT state in question should become trivial if it is "lifted" or "pulled back" from $G$ to $H$, or more concretely if certain fields are regarded as elements of $H$ rather than as elements of $G$.)

From a mathematical point of view, this gives another choice in the usual paradigm that says that the boundary of a SPT phase is gapless, has topological order on the boundary, or breaks the symmetry. Another possibility is that the global symmetry of the bulk SPT phase might be extended (or enhanced) to a larger group along the boundary, satisfying certain conditions. In $1+1$ dimensions, this is a standard result: The usual symmetry-preserving boundaries of $(1+1)$-dimensional bulk SPT phases have a group extension along the boundary. The novelty is that a gapped boundary can be achieved above $1+1$ dimensions via such a group extension.

Let us pause to explain more fully the assertion that what we have just described extends a standard $(1+1)$ dimensional phenomenon to higher dimensions. In the usual formulation of the $(1+1)$-dimensional Haldane or Affleck-Lieb-Kennedy-Tasaki (AKLT) spin chain, one considers a chain of spin-1 particles with $S O(3)$ symmetry. The boundary is not gapped and carries spin $1 / 2$. Alternatively, one could attach a spin- $1 / 2$ particle to each end of such a chain. Then the system can be gapped, with a unique ground state, but the global symmetry is extended from $S O(3)$ to $S U(2)$ at the ends of the chain. What we have described is an analog of such a symmetry extension in $2+1$ dimensions.

In general, a bulk SPT state protected by a symmetry $G$ can also be viewed as a many-body state with a symmetry $H$, where the subgroup $K$ acts trivially in the bulk (i.e., the bulk degrees of freedom are singlets of $K$ ). For example, we may view the CZX model to have a $Z_{4}^{H}$-symmetry in the bulk. By definition, two states in two different $G$-SPT phases cannot smoothly deform into each other via deformation paths that preserve the $G$-symmetry. However, two such $G$-SPT states may be able to smoothly deform into each other if we view them as systems with the extended $H$-symmetry and deform them along the paths that preserve the $H$-symmetry. For example, the nontrivial $Z_{2}^{G}$-SPT state of the CZX model can smoothly deform into the trivial $Z_{2}^{G}$-SPT state along a deformation path that preserves the 
extended $Z_{4}^{H}$-symmetry. In other words, when viewed as a $Z_{4}^{H}$ symmetric state, the ground state of the CZX model has a trivial $Z_{4}^{H}$-SPT order. Since it has a trivial $Z_{4}^{H}$-SPT order, it is not surprising that the CZX model can have a gapped boundary that preserves the extended $Z_{4}^{H}$-symmetry, as explicitly constructed above. In general, if two $G$-SPT states are connected by an $H$-symmetric deformation path, then we can always construct an $H$-symmetric domain wall between them by simply using the $H$-symmetric deformation path. This is the physical meaning behind a $G$-SPT state having a gapped boundary with an extended symmetry $H$.

From the point of view of condensed matter physics, however, the sort of gapped boundary that we have described so far will generally not be physically sensible. Microscopically, condensed matter systems generally do not have extra symmetries that act only along their boundary. (There can be exceptions like the case just mentioned, which is conceivable in any dimension: a system that, in bulk, is made from particles of integer spin but has half-integer spin particles attached on the surface. Then, a $2 \pi$ rotation of the spins is nontrivial only along the boundary.)

In a system microscopically without an extended symmetry along the boundary, one might be tempted to interpret $K$ as a group of emergent global symmetries, not present microscopically. But there is a problem with this. In condensed matter physics, one may often run into emergent global symmetries in a low-energy description. But these are always approximate symmetries, explicitly broken by operators that are irrelevant at low energies in the renormalization group sense.

That is not viable in the present context. Since the global symmetry that is generated by $U_{\mathrm{CZX}}$ is supposed to be an exact symmetry, we cannot explicitly violate the boundary symmetry group generated by $U_{\mathrm{CZX}}^{2}$. Obviously, any interaction that is not invariant under $U_{\mathrm{CZX}}^{2}$ is also not invariant under $U_{\mathrm{CZX}}$.

What we can do instead is to gauge the boundary symmetry group $K$. Then, the global symmetry group that acts on gauge-invariant operators and on physical states is just the original group $H / K=G$. This way, we do not break or extend the symmetry on the boundary. Since $K$ is an on-site symmetry group, there is no difficulty in gauging it; we explain two approaches in Secs. III C and III D.

In $3+1$ (or more) dimensions, a procedure along these lines starting with a bulk SPT phase with symmetry group $G$ and a group extension as in Eq. (3.7) that satisfies the appropriate cohomological condition will lead to a gapped boundary state with topological order along the boundary. The topological order is a version of gauge theory with gauge group $K$ (possibly twisted by a cocycle). We will give a general description of such gapped boundary states in Sec. IX. In $2+1$ dimensions, the boundary has dimension $1+1$ and one runs into the fact that topological order is not possible in $1+1$ dimensions. As a result, what we will actually get in the CZX model by gauging the boundary symmetry $K$ is not really a fundamentally new boundary state.

\section{The third boundary of the CZX model: Lattice $Z_{2}^{K}$-gauge theory on the boundary}

We will describe two ways to gauge the boundary symmetry $K=Z_{2} \equiv Z_{2}^{K}$. The most straightforward way, although as we will discuss ultimately less satisfactory for condensed matter physics, is to simply incorporate a boundary gauge field.

As indicated in Fig. 5, we label the link between boundary sites $i$ and $i+1$ by the half-integer $i+\frac{1}{2}$. Placing a $Z_{2}$-valued gauge field on this link means introducing a qubit associated to this link with operators $V_{i+\frac{1}{2}}, E_{i+\frac{1}{2}}$ that obey

$$
V_{i+\frac{1}{2}}^{2}=E_{i+\frac{1}{2}}^{2}=1, \quad E_{i+\frac{1}{2}} V_{i+\frac{1}{2}}=-V_{i+\frac{1}{2}} E_{i+\frac{1}{2}} .
$$

Here, $V_{i+\frac{1}{2}}$ describes parallel transport between sites $i$ and $i+1$, and $E_{i+\frac{1}{2}}$ is a discrete electric field that flips the sign of $V_{i+\frac{1}{2}}$.

Now, let us discuss the gauge constraint at site $i$. A gauge transformation that acts at site $i$ by the nontrivial element in $Z_{2}^{K}$ is supposed to flip the signs of $V_{i \pm \frac{1}{2}}$, the holonomies on the two links connecting to site $i$. To do this, it will have a factor $E_{i+\frac{1}{2}} E_{i-\frac{1}{2}}$. It should also act on the spins as $U_{\mathrm{CZX}, i}^{2}=-\sigma_{i+}^{z} \sigma_{i-}^{z}$. Thus, the gauge generator on site $i$ is

$$
\Omega_{i}=E_{i+\frac{1}{2}} E_{i-\frac{1}{2}} U_{\mathrm{CZX}, i}^{2} .
$$

A physical state $|\Psi\rangle$ in the gauge theory must be gauge invariant; that is, it must obey

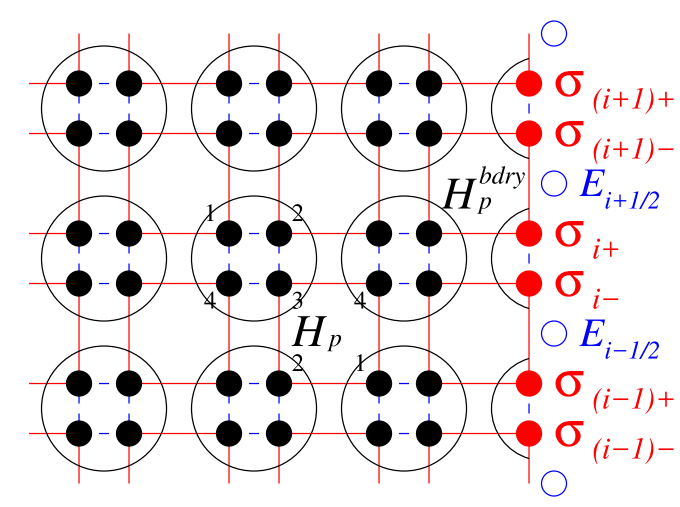

FIG. 5. Gauging the boundary symmetry $K=Z_{2} \equiv Z_{2}^{K}$ of the boundary state of Fig. 4 is accomplished by placing on each boundary link a $Z_{2}$-valued gauge field. We label the link between boundary sites $i$ and $i+1$ by the half-integer $i+\frac{1}{2}$. We associate to this link a new qubit with a discrete holonomy (as discussed in the text) and a discrete electric field $E_{i+\frac{1}{2}}$. 


$$
\Omega_{i}|\Psi\rangle=|\Psi\rangle
$$

However, as $E_{i+\frac{1}{2}}^{2}=1$ for all $i$, if we take the product of $\Omega_{i}$ over all boundary sites, the factors of $E_{i+\frac{1}{2}}$ cancel out, and we get

$$
\prod_{i} \Omega_{i}=\prod_{i} U_{\mathrm{CZX}, i}^{2}
$$

Hence, Eq. (3.10) implies that a physical state $|\Psi\rangle$ satisfies

$$
\prod_{i} U_{\mathrm{CZX}, i}^{2}|\Psi\rangle=|\Psi\rangle
$$

But this precisely means that a physical state is invariant under the global action of $K$, so that the global symmetry group that acts on the system reduces to the original global symmetry $G$.

The Hamiltonian $H=\sum H_{p}$ must be slightly modified to be gauge invariant, that is, to commute with $\Omega_{i}$. To see the necessary modification, let us look at the plaquette Hamiltonian $H_{p}$ for the boundary plaquette shown in the figure, which contains the boundary link labeled $i+\frac{1}{2}$. $H_{p}$ as defined in Eq. (2.9) anticommutes with $\Omega_{i}$ and $\Omega_{i+1}$ because the operator $|\uparrow \uparrow \uparrow \uparrow\rangle\langle\downarrow \downarrow \downarrow \downarrow|+| \downarrow \downarrow \downarrow \downarrow\rangle\langle\uparrow \uparrow \uparrow \uparrow|$ has that property. (It flips one of the spins at boundary site $i$ and one at boundary site $i+1$, so it anticommutes with $U_{\mathrm{CZX}, i}^{2}=-\sigma_{i+}^{z} \sigma_{i-}^{z}$ and similarly with $U_{\mathrm{CZX}, i+1}^{2}$.) To restore gauge invariance is surprisingly simple: We just have to multiply $H_{p}$ by $V_{i+\frac{1}{2}}$, which also anticommutes with $\Omega_{i}$ and $\Omega_{i+1}$. So, we can take the Hamiltonian for a boundary plaquette containing the boundary link $i+\frac{1}{2}$ to be

$$
\begin{gathered}
H_{p, i+\frac{1}{2}}^{\mathrm{bdry}}=-(|\uparrow \uparrow \uparrow \uparrow\rangle\langle\downarrow \downarrow \downarrow \downarrow|+| \downarrow \downarrow \downarrow \downarrow\rangle\langle\uparrow \uparrow \uparrow \uparrow|) \\
\otimes V_{i+\frac{1}{2}} \otimes_{\alpha} P_{p}^{\alpha} .
\end{gathered}
$$

For a gauge-invariant and $G$-invariant Hamiltonian, we can take the sum of all bulk and boundary plaquette Hamiltonians.

This Hamiltonian $H$ commutes with all the discrete gauge fields $V_{i+\frac{1}{2}}$, so, in looking for an eigenstate of $H$ (ignoring for a moment the gauge constraint), we can specify arbitrarily the eigenvalues of the $V$ 's. Let $\left|v_{i+\frac{1}{2}}\right\rangle$ be a state of the gauge fields with eigenvalue $v_{i+\frac{1}{2}}$ for $V_{i+\frac{1}{2}}$. (Of course, these eigenvalues are \pm 1 since $V_{i+\frac{1}{2}}^{2}=1$.) The ground state of $H$ with these eigenvalues of the $V_{i+\frac{1}{2}}$ is simply

$$
\underset{\text { bulk }}{\otimes} \frac{|\uparrow \uparrow \uparrow \uparrow\rangle+|\downarrow \downarrow \downarrow \downarrow\rangle}{\sqrt{2}} \otimes \frac{|\uparrow \uparrow \uparrow \uparrow\rangle+V_{i+\frac{1}{2}}|\downarrow \downarrow \downarrow \downarrow\rangle}{\sqrt{2}} \otimes\left|v_{i+\frac{1}{2}}\right\rangle .
$$

Let us denote this state as $\left.\left.\| v_{i+\frac{1}{2}}\right\rangle\right\rangle$. If the boundary has $L$ links, there are $2^{L}$ of these states.

The states $\left.\left.\| v_{i+\frac{1}{2}}\right\rangle\right\rangle$ are degenerate, and these are the ground states of $H$. However, to make states that satisfy the gauge constraint, we must take linear combinations of the $\left.\| v_{i+\frac{1}{2}}\right\rangle$. Since a gauge transformation at site $i$ flips the signs of $v_{i \pm \frac{1}{2}}$, the only gauge-invariant function of the $v_{i+\frac{1}{2}}$ is their product. Assuming that the boundary is compact and, thus, is a circle, this product is the holonomy of the $Z_{2}^{K}$ gauge field around the circle. (With periodic boundary conditions along the boundary, there are no corners along the boundary circle; otherwise, our discussion can be slightly modified to incorporate corners.) Thus, there are two gauge-invariant ground states, depending on the sign of the holonomy $\prod_{i} v_{i+\frac{1}{2}}$. They are

$$
\left.\left.\left|\Psi_{\mathrm{gS}}(+)\right\rangle=\sum_{\left\{v_{i+\frac{1}{2}}\right\}, \prod_{i} v_{i+\frac{1}{2}}=1} c_{\left\{v_{i+\frac{1}{2}}\right\}} \| v_{i+\frac{1}{2}}\right\rangle\right\rangle
$$

and

$$
\left.\left.\left|\Psi_{\mathrm{gs}}(-)\right\rangle=\sum_{\left\{v_{i+\frac{1}{2}}\right\}, \prod_{i} v_{i+\frac{1}{2}}=-1} c_{\left\{v_{i+\frac{1}{2}}\right.} \| v_{i+\frac{1}{2}}\right\}\right\rangle .
$$

[Here, the signs $c_{\left\{v_{i+\frac{1}{2}}\right\}}= \pm 1$ are determined by the gauge constraints. With our choice of sign in the gauge constraints $\Omega_{i}$, flipping two of the $v_{i}$ that are separated by $n$ lattice states multiplies the amplitude by $(-1)^{n}$. This could be avoided by changing the sign of $\Omega_{i}$, but that creates complications elsewhere.]

Now, let us study the transformation of these states under the global symmetry group $G=Z_{2} \equiv Z_{2}^{G}$. When we apply $U_{\mathrm{CZX}}$ to the states $\left|\Psi_{\mathrm{gs}}( \pm)\right\rangle$, we find that all the sign factors $C Z_{i j}$ cancel each other. This occurs by the same cancellation as in the original bulk version of the CZX model. However, the wave function is no longer trivially invariant under flipping the spins; rather, the wave function $|\uparrow \uparrow \uparrow \uparrow\rangle+V_{i+\frac{1}{2}}|\downarrow \downarrow \downarrow \downarrow\rangle$ for a boundary plaquette is multiplied by $V_{i+\frac{1}{2}}$ when the spins in this plaquette are flipped. So, taking into account all the boundary plaquettes,

$$
U_{\mathrm{CZX}}\left|\Psi_{\mathrm{gs}}( \pm)\right\rangle= \pm\left|\Psi_{\mathrm{gs}}( \pm)\right\rangle
$$

Thus, the transformation of a state under the global symmetry $Z_{2}^{G}$ is locked to its holonomy under the gauge symmetry $Z_{2}^{K}$.

The formula in Eq. (3.17) has been written as if the boundary of the system consists of a single circle; for example, the spatial topology may be a disc. More generally, we can consider a system whose boundary consists of several circles. Each boundary component has its own 
$Z_{2}^{K}$-valued holonomy, and the action of $U_{\mathrm{CZX}}$ on a ground state is the product of all of these holonomies.

Now, let us look for a local operator with a nonzero matrix element between the two ground states $\left|\Psi_{\mathrm{gs}}( \pm)\right\rangle$. For this, we need first of all an operator that changes the sign of the holonomy around the boundary. The simplest operator with this property is simply $E_{i+1 / 2}$ (for some $i$ ). Because it flips the sign of $V_{i+1 / 2}$, it reverses the sign of the holonomy. However, the operator $E_{i+1 / 2}$ is invariant under the global symmetry group $Z_{2}^{G}$, and therefore, it cannot possibly have a nonzero matrix element between the two ground states, which transform oppositely under the global symmetry.

Concretely, $E_{i+1 / 2}$ does not map $\left|\Psi_{\mathrm{gs}}( \pm)\right\rangle$ to $\left|\Psi_{\mathrm{gs}}(\mp)\right\rangle$ because it anticommutes with $V_{i+1 / 2}$, which appears in one factor in the definition of the state $\left.\left.\| v_{i+1 / 2}\right\rangle\right\rangle$ in Eq. (3.14), namely,

$$
|\uparrow \uparrow \uparrow \uparrow\rangle+V_{i+1 / 2}|\downarrow \downarrow \downarrow \downarrow\rangle .
$$

[Instead, $E_{i+1 / 2}\left|\Psi_{\mathrm{gs}}(+)\right\rangle$ is a new state that has the same holonomy as $\left|\Psi_{\mathrm{gs}}(-)\right\rangle$, but differs from it by the presence of an additional quasiparticle carrying a nontrivial global $Z_{2}^{G}$-charge localized near the link at $i+1 / 2$.] However, we can get a local operator that reverses the holonomy and commutes with this $V_{i+1 / 2}$ if we just replace $E_{i+1 / 2}$ by

$$
X_{i+1 / 2}=E_{i+1 / 2} \sigma_{i+}^{z} .
$$

(We could equally well use $\sigma_{i+1-}^{z}$ instead of $\sigma_{i+}^{z}$.) This operator leaves invariant the expression in Eq. (3.18) and, accordingly, it simply exchanges the states $\left|\Psi_{\mathrm{gs}}( \pm)\right\rangle$ :

$$
X_{i+1 / 2}\left|\Psi_{\mathrm{gs}}( \pm)\right\rangle=\left|\Psi_{\mathrm{gs}}(\mp)\right\rangle \text {. }
$$

The operator $X_{i+1 / 2}$ is odd under the global $Z_{2}^{G}$ symmetry, because of the factor of $\sigma_{i+}^{z}$. This, of course, is consistent with the fact that this operator exchanges the states $\left|\Psi_{\mathrm{gs}}( \pm)\right\rangle$. However, the existence of a $Z_{2}^{G}$-odd local operator that exchanges the two ground states means that we must interpret the boundary state that we have constructed as one in which the global $Z_{2}^{G}$-symmetry is spontaneously broken along the boundary. Indeed, although $\left\langle\Psi_{\mathrm{gs}}(+)\left|X_{i+1 / 2}\right| \Psi_{\mathrm{gs}}(+)\right\rangle=0$, the two-point function of the operator $X_{i+1 / 2}$ in the state $\left|\Psi_{\mathrm{gs}}(+)\right\rangle$ exhibits the long-range order that signals the $Z_{2}^{G}$-spontaneous symmetry breaking. In fact,

$$
\left\langle\Psi_{\mathrm{gs}}(+)\left|X_{i+1 / 2} X_{j+1 / 2}\right| \Psi_{\mathrm{gs}}(+)\right\rangle=1
$$

for any $i, j$. Similarly, $\left\langle\Psi_{\mathrm{gs}}(-)\left|X_{i+1 / 2} X_{j+1 / 2}\right| \Psi_{\mathrm{gs}}(-)\right\rangle=1$.

This result is somewhat disappointing, since it is certainly already known that any SPT phase in any dimension can have a gapped boundary state in which the symmetry is explicitly or spontaneously broken. However, as we will see starting in Sec. IV, similar gapped boundary states can be constructed for SPT phases in any dimension, and in $3+1$ (or more) dimensions, the gapped boundary states constructed this way are genuinely novel: They have topological order along the boundary, rather than symmetry breaking. What we have run into here is that the $(1+1)$-dimensional boundary of a $(2+1)$-dimensional system does not really support topological order. Discrete gauge symmetry (such as the $Z_{2}^{K}$ considered here) can describe topological order in dimensions $\geq 2+1$, but not in $1+1$ dimensions.

By contrast, the gapped boundary state described in Sec. III B, in which the symmetry is extended along the boundary rather than being spontaneously broken, is genuinely new even in $2+1$ dimensions. But as we have noted, such a symmetry extension along the boundary is physically sensible in condensed matter physics only in particular circumstances.

Going back to the case that the boundary symmetry is gauged, where does the state that we have described fit into the usual classification of gapped phases of discrete gauge theories? Since the states $\left|\Psi_{\mathrm{gs}}( \pm)\right\rangle$ with opposite holonomies are degenerate, this would usually be called a deconfined phase. But it differs from a standard deconfined phase in the following way. Typically, in $(1+1)$ dimensional gauge theory with a discrete gauge group, the degeneracy between states with different holonomy can be lifted by a suitable perturbation such as

$$
-u \sum_{i} E_{i+1 / 2}
$$

with a constant $u$ (or, more generally, $-\sum_{i} u_{i} E_{i+1 / 2}$ with any small parameters $u_{i}$; a small local perturbation is enough). In an ordinary $Z_{2}^{K}$ gauge theory, such a term would induce an effective Hamiltonian density $-u\left(\begin{array}{ll}0 & 1 \\ 1 & 0\end{array}\right)$ acting on the two states $\left(\begin{array}{c}\Psi_{\mathrm{gs}}(+) \\ \Psi_{\mathrm{gs}}(-)\end{array}\right)$. The ground state would then be (for $u>0$ ) a superposition of $\left|\Psi_{\mathrm{gs}}(+)\right\rangle$ and $\left|\Psi_{\mathrm{gs}}(-)\right\rangle$. A discrete gauge theory with a nondegenerate ground state that involves such a sum over holonomies is said to be confining.

In the present context, the global $Z_{2}^{G}$-symmetry under which the states $\left|\Psi_{\mathrm{gs}}( \pm)\right\rangle$ transform oppositely prevents such an effect. On the contrary, it ensures that the degeneracy among these two states cannot be lifted by any local perturbation that preserves the $Z_{2}^{G}$-symmetry. The above remarks demonstrating the spontaneous breaking of the global $Z_{2}^{G}$-symmetry make the issue clear. The spontaneously broken symmetry leads to a twofold degeneracy of the ground state that is exact in the limit of a large system.

The remarks that we have just made have obvious analogs in the construction described in the emergent gauge theory construction of Sec. III D, and they will not be repeated there. 


\section{The fourth boundary of the CZX model: Emergent lattice $Z_{2}^{K}$-gauge theory on the boundary}

The model constructed in Sec. III C using lattice $Z_{2}^{K}$ gauge fields reduces the global symmetry to the original $Z_{2}^{G}$. However, it has one flaw from the point of view of condensed matter physics. In condensed matter physics, not only are the symmetries on-site, but more fundamentally the Hilbert space can be assumed to be on-site: that is, the full Hilbert space is a tensor product of local factors, one for each site. (In fact, the Hilbert space has to be on-site before it makes sense to say that the symmetries are on-site.)

The purpose of the present section is to explain how to construct a model with on-site Hilbert space and symmetries that has the same macroscopic behavior as found in Sec. III C.

The reason that the model in Sec. III C does not have this property is that the variables $V_{i+\frac{1}{2}}$ and $E_{i+\frac{1}{2}}$ are associated to boundary links, not to boundary sites. One could try to cure this problem by associating these link variables to the site just above (or just below) the link in question. The trouble with this is that then, although the full Hilbert space is onsite, the gauge-symmetry generators $\Omega_{i}$ are not on-site (they involve operators acting at two adjacent sites). Accordingly, the space of physical states, invariant under the $\Omega_{i}$, is not an on-site Hilbert space.

By analogy with various constructions in condensed matter physics, one might be tempted to avoid this problem by relaxing the physical state constraint $\Omega_{i}|\Psi\rangle=|\Psi\rangle$ and instead adding to the Hamiltonian a term

$$
\Delta H=-c \sum_{i} \Omega_{i},
$$

with a positive constant $c$. Then, minimum energy states satisfy the constraint $\Omega_{i}|\Psi\rangle=|\Psi\rangle$ as assumed in Sec. III C, and, on the other hand, the full Hilbert space and the global $Z_{2}^{G}$-symmetry are on-site.

In the present context, this approach is not satisfactory. Once we relax the constraint that physical states are invariant under $\Omega_{i}$, the global symmetry of the model is extended along the boundary from $G=Z_{2}^{G}$ to $H=Z_{4}^{H}$, and we have really not gained anything by adding the gauge fields.

Instead, what we have to do is to replace the "elementary" $Z_{2}^{\prime}=Z_{2}^{K}$ gauge fields of Sec. III C by "emergent" gauge fields, by which we mean simply gauge fields that emerge in an effective low-energy description from a microscopic theory with an on-site Hilbert space. There are many ways to do this, and it does not matter exactly which approach we pick. In this section, we will describe one simple approach.

We start with the boundary obtained in Sec. III B and add to each boundary site a pair of qubits described by Pauli matrices $\tau_{i_{+}}$(see Fig. 6). Since each boundary site already contained the two qubits $\sigma_{i \pm}$, this gives a total of four qubits in each boundary site, and a local Hilbert space $\mathcal{H}_{i}^{0}$ of

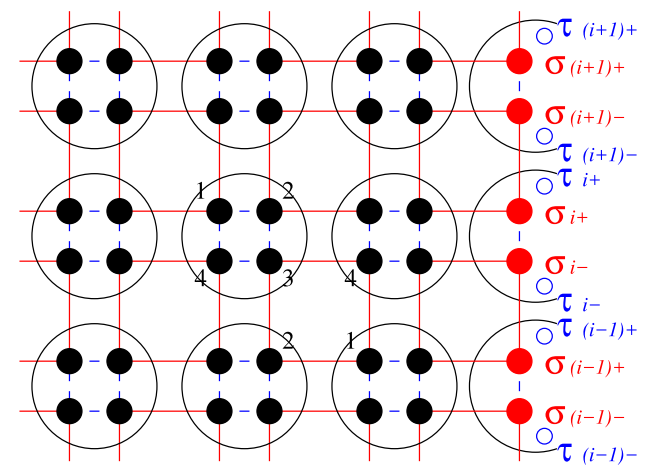

FIG. 6. The filled dots are qubits (or spin-1/2's). A (half-)circle (with dots inside) represents a site. The dashed blue line connecting dots $i, j$ represents the phase factor $C Z_{i j}$ in the $Z_{2}^{G}$ or $Z_{4}^{H}$ global symmetry transformation. The open dots on the boundary are the $Z_{2}^{\prime} \equiv Z_{2}^{K}$-gauge degrees of freedom $E_{i+\frac{1}{2}}$.

dimension $2^{4}$. However, we define the Hilbert space $\mathcal{H}_{i}$ of the $i$ th boundary site to be the subspace of $\mathcal{H}_{i}^{0}$ of states that satisfy the local gauge constraint

$$
\hat{U}_{i}^{\text {gauge }}|\Psi\rangle=|\Psi\rangle,
$$

where

$$
\hat{U}_{i}^{\text {gauge }}=-\sigma_{i_{+}}^{z} \sigma_{i_{-}}^{z} \tau_{i_{+}}^{z} \tau_{i_{-}}^{z} .
$$

The constraint is on-site, so $\mathcal{H}_{i}$ is on-site.

Now, we add to the Hamiltonian a gauge-invariant boundary perturbation

$$
-U \sum_{i} \tau_{i_{+}}^{z} \tau_{(i+1)_{-}}^{z}
$$

with a large positive coefficient $U$. At low energies, this will lock $\tau_{i_{+}}^{z}=\tau_{(i+1)_{-}}^{z}$. In this low-energy subspace, $\tau_{i_{+}}^{z}=$ $\tau_{(i+1)_{-}}^{z}$ will play the role of $E_{i+\frac{1}{2}}$ in the last subsection. What will now play the role of the conjugate gauge field is

$$
V_{i+\frac{1}{2}}=\tau_{i_{+}}^{x} \tau_{(i+1)_{-}}^{x},
$$

which anticommutes with $\tau_{i_{+}}^{z}=\tau_{(i+1)_{-}}^{z}$. The Hamiltonian for a boundary plaquette is defined as in Eq. (3.13), but with this "composite" definition of $V_{i+\frac{1}{2}}$, and it commutes with the gauge constraint operator in Eq. (3.25).

The global $Z_{2}$-symmetry generator on the $i$ th boundary site is now given by

$$
\hat{U}_{Z_{2}, i}=\sigma_{i_{-}}^{x} \sigma_{i_{+}}^{x} U_{C Z, i_{-}, i_{+}} \mathrm{e}^{\mathrm{i} \frac{\pi}{4} \tau_{i_{-}}^{z}} \mathrm{e}^{-\mathrm{i} \frac{\pi}{4} \tau_{i_{+}}^{z}} .
$$

We find that

$$
\hat{U}_{Z_{2}, i}^{2}=-\sigma_{i_{-}}^{z} \sigma_{i_{+}}^{z} \tau_{i_{+}}^{z} \tau_{i_{-}}^{z}=\hat{U}_{i}^{\text {gauge }}
$$


So, $\hat{U}_{Z_{2}, i}^{2}=1$ on states that satisfy the gauge constraint. This is true for every bulk or boundary state, so the full global symmetry generator, obtained by taking the product of the symmetry generators over all bulk or boundary sites, generates the desired symmetry group $Z_{2}^{G}$.

The low-energy dynamics can be analyzed precisely as in Sec. III C, and with the same results. The first step is to observe that, even in the presence of the perturbation of Eq. (3.26), the Hamiltonian commutes with the operators $V_{i+\frac{1}{2}}$. Just as in Sec. III C, one diagonalizes these operators with eigenvalues $v_{i+\frac{1}{2}}$, finds the ground state for given $v_{i+\frac{1}{2}}$, and then takes linear combinations of these states to satisfy the gauge constraint.

We remind the readers that Appendix A of this paper contains more details on boundaries of the CZX model and their $1+1 \mathrm{D}$ boundary effective theories. For a fermionic version of the CZX model, see Appendix B. The boundary of the fermionic CZX model with emergent $Z_{2}^{K}$-gauge theory with anomalous global symmetry is detailed in Appendix C.

For the generalization of what we have done to arbitrary SPT phases in any dimension, we can now proceed to Sec. IV.

\section{BOUNDARIES OF GENERIC SPT STATES IN ANY DIMENSION}

What we have done for the CZX model in $2+1$ dimensions has an analog for a general SPT state in any dimension. To explain this will require a more abstract approach. We work in the framework of the group cohomology approach to SPT states, with a Lagrangian on a spacetime lattice. So we first introduce our notation for that subject. We generically write $\nu_{d}$ for a homogeneous $d$-cocycle and $\mu_{d}$ for a homogeneous $d$-cochain. We similarly write $\omega_{d}$ for an inhomogeneous $d$-cocycle and $\beta_{d}$ for an inhomogeneous $d$-cochain. Finally, we write $\mathcal{V}_{d}$ for homogeneous $d$-cocycles or $d$-cochains with both global symmetry variables and gauge variables and denote $\Omega_{d}$ as inhomogeneous $d$-cocycles or $d$-cochains with both global symmetry variables and gauge variables.

\section{A. An exactly soluble path integral model that realizes a generic SPT state}

A generic SPT state with a finite symmetry group $G$ can be described by a path integral on a space-time lattice, or more precisely, a space-time complex with a branching structure. A branching structure can be viewed as an ordering of all vertices. It gives each link an orientation-which we can think of as an arrow that runs from the smaller vertex on that link to the larger one, as in Fig. 7. More generally, a branching structure determines an orientation of each $k$-dimensional simplex, for every $k$, including the topdimensional ones that are glued together to make the full spacetime.

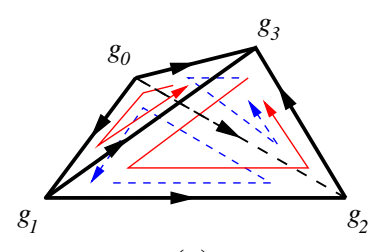

(a)

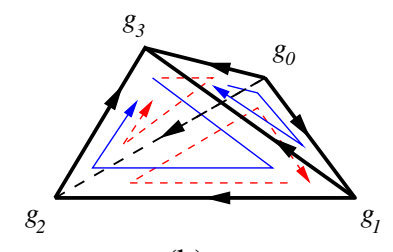

(b)
FIG. 7. The triangles with red (blue) loops have positive orientation $s_{i j k}=1$ (negative orientation $s_{i j k}=-1$ ), with an outward (inward) area vector through the right-hand rule. The orientation of a tetrahedron (i.e., the three-simplex) is determined by the orientation of the triangle not containing the first vertex. So, (a) has a positive orientation $s_{01234}=+1$, and (b) has a negative orientation $s_{01234}=-1$.

To each vertex $i$, we attach a $G$-valued variable $g_{i}$. (Later, we may also assign group elements $g_{i j}$ to each edges $\overline{i j}$.) An assignment of group elements to vertices or edges will be called a "coloring." For a discrete version of the usual path integral of quantum mechanics, we will sum over all the colorings. (See Sec. IX A.) On a closed oriented spacetime, the "integrand" of the path integral is given by

$$
e^{-\int_{M^{3}} \mathcal{L}_{\mathrm{Bulk}} d^{3} x}=\prod_{M^{3}} \nu_{3}^{s_{i j k l}}\left(g_{i}, g_{j}, g_{k}, g_{l}\right)
$$

The argument of the path integral is a complex number with a nontrivial phase and, thus, it can produce complex Berry phases. We have written this formula for the case of $2+1$ dimensions, but it readily generalizes to any dimension. Here, $s_{i j k l}= \pm 1$ for a given simplex with vertices $i j k l$, depending on whether the orientation of that simplex that comes from the branching structure agrees or disagrees with the orientation of $M$. The symbol $\prod_{M^{3}}$ represents a product over all $d$-simplices.

Finally, and most importantly, the $U(1)$-valued $\nu_{d}\left(g_{0}, \ldots, g_{d}\right)$ is a homogeneous cocycle representing an element of $\mathcal{H}^{d}[G, U(1)]$. This means $\nu_{d}\left(g_{0}, \ldots, g_{d}\right)$ satisfy the cocycle condition $\delta \nu_{d}=1$, where

$$
\left(\delta \nu_{d}\right)\left(g_{0}, \ldots, g_{d+1}\right) \equiv \frac{\prod_{i=\mathrm{even}} \nu_{d}\left(g_{0}, \ldots, \hat{g}_{i}, \ldots, g_{d+1}\right)}{\prod_{i=\mathrm{odd}} \nu_{d}\left(g_{0}, \ldots, \hat{g}_{i}, \ldots, g_{d+1}\right)} .
$$

(The symbol $\hat{g}_{i}$ is an instruction to omit $g_{i}$ from the sequence.)

We regard the complex phase $\nu_{d}^{s}$ as a quantum amplitude assigned to a $d$-simplex in a $d$-dimensional spacetime.

First, the path-integral model defined by the action amplitude Eq. (4.1) has a $G$-symmetry

$$
\begin{aligned}
\prod_{M^{3}} \nu_{3}^{s_{i j k l}}\left(g_{i}, g_{j}, g_{k}, g_{l}\right) & =\prod_{M^{3}} \nu_{3}^{s_{i j k l}}\left(g g_{i}, g g_{j}, g g_{k}, g g_{l}\right), \\
g & \in G,
\end{aligned}
$$


since the homogeneous cocycle satisfies

$$
\nu_{3}\left(g_{i}, g_{j}, g_{k}, g_{l}\right)=\nu_{3}\left(g g_{i}, g g_{j}, g g_{k}, g g_{l}\right)
$$

Second, because of the cocycle condition, one can show that

$$
e^{-\int_{M^{3}} \mathcal{L}_{\text {Bulk }} d^{3} x}=\prod_{M^{3}} \nu_{3}^{s_{i j k l}}\left(g_{i}, g_{j}, g_{k}, g_{l}\right)=1,
$$

for any set of $g$ 's, when the spacetime $M^{3}$ is an orientable closed manifold. This implies that the model is trivially soluble on a closed spacetime and describes a state in which all local operators have short-range correlations. This state is symmetric and gapped. It realizes a SPT state with symmetry $G$. The state is determined up to equivalence by the cohomology class of $\nu_{3}$.

\section{B. The first boundary of a generic SPT state: A simple model but with complicated boundary dynamics}

So far, we have described a discrete system with $G$-symmetry on a closed three-manifold $M^{3}$. What happens if $M^{3}$ is an open manifold that has a boundary $\partial M^{3}=M^{2}$ ? The simplest path-integral model that we can construct is simply to use all of the above formulas, but now, on a manifold with a boundary. Thus, the argument of the path integral is still given by Eq. (4.1), but now, this is no longer trivial:

$$
e^{-\int_{M^{3}} \mathcal{L}_{\text {Bulk }} d^{3} x}=\prod_{M^{3}} \nu_{3}^{s_{i j k l}}\left(g_{i}, g_{j}, g_{k}, g_{l}\right) \neq 1
$$

Because of the properties of the cocycle, this amplitude only depends on the $g_{i}$ on the boundary, so it can be viewed as the integrand of the path integral of a boundary theory.

To calculate the path integral amplitude of the boundary theory, we can simplify the bulk so that it contains only one vertex $g^{*}$ (see Fig. 8). In this case, the effective boundary theory is described by a path integral based on the following amplitude:
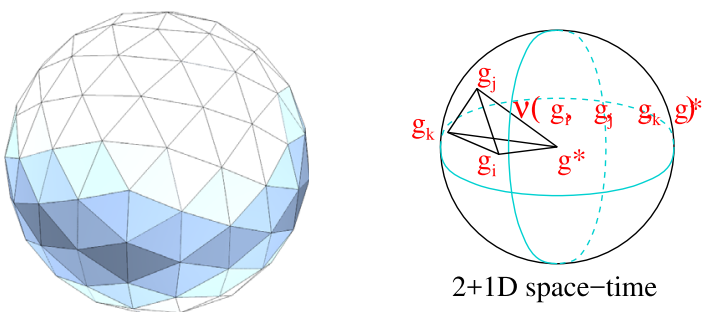

FIG. 8. The space-time $D^{3}$, with a triangulation of the boundary and a construction of three-simplices (or four-cells) in the bulk. Such a triangulation is used to construct a low-energy effective path integral for the boundary.

$$
e^{-\int_{\partial M^{3}} \mathcal{L}_{\mathrm{Bdry}, \partial M^{3} d^{2} x}}=\prod_{\partial M^{3}} \nu_{3}^{s_{i j k}}\left(g_{i}, g_{j}, g_{k}, g^{*}\right) .
$$

This depends only on the boundary spins $g_{i}, g_{j}, g_{k}, \ldots$, and not on $g^{*}$ in the bulk. (This follows from the cocycle condition for $\nu_{3}$. Readers who are not familiar with this statement can find the proof in Sec. IX.) Here, $s_{i j k}= \pm 1$ depending on whether the orientation of a given triangle that comes from the branching structure agrees with the orientation that comes from the triangle as part of the boundary of the oriented manifold $M^{3}$. [Symbols like $d^{3} x$ and similar notation below are shorthands for products over simplices, as written explicitly in the right-hand side of Eq. (4.7).]

Since the path integral amplitude of the boundary theory is path dependent and not equal to 1, the dynamics of the simple model is hard to solve, and we do not know if the boundary is gapped, symmetry breaking, or topological. In fact, for cocycles $\nu_{3}$ that are in the same equivalence class but differ by coboundaries, the boundary amplitudes are different, which may lead to different boundary dynamics. In Sec. III A, for the case of the CZX model, we have chosen a particular cocycle in an equivalence class. This choice of cocycle leads to a gapless boundary.

In general, given only a generic cocycle, the dynamics of this model is unclear and possibly nonuniversal. We will describe more fully the anomalous symmetry realization in this boundary state in Sec. IV C, and then we will introduce alternative boundary states in Sec. IV D.

\section{Non-on-site (anomalous) $G$-symmetry transformation on the boundary effective theory \\ 1. Symmetry transformation on a spacetime boundary in Lagrangian formalism}

We continue to assume that the spacetime manifold $M^{3}$ has a boundary $\partial M^{3}=M^{2}$, which can be regarded as a fixed-time slice on the closed space region $\partial M^{3}$. The effective theory Eq. (4.7) possesses the $G$-symmetry:

$$
\begin{aligned}
e^{-\int_{\partial M^{3}} \mathcal{L}_{\text {Bdry }, \partial M^{3}} d^{2} x} & =\prod_{\partial M^{3}} \nu_{3}^{s_{i j k}}\left(g_{i}, g_{j}, g_{k}, g^{*}\right) \\
& =\prod_{\partial M^{3}} \nu_{3}^{s_{i j k}}\left(g g_{i}, g g_{j}, g g_{k}, g^{*}\right) .
\end{aligned}
$$

But this $G$-symmetry in the presence of a boundary is in fact anomalous (i.e., non-on-site). The anomalous nature of the symmetry along the boundary is the most important property of SPT states.

To understand such an anomalous (or non-on-site) symmetry, we note that, locally (that is, for a particular simplex), the action amplitude is not invariant under the $G$-symmetry transformation: 


$$
\nu_{3}\left(g g_{i}, g g_{j}, g g_{k}, g^{*}\right) \neq \nu_{3}\left(g_{i}, g_{j}, g_{k}, g^{*}\right) .
$$

Only the total action amplitude on the whole boundary (here, the boundary $\partial M^{3}=M^{2}$ of an open manifold is a closed manifold) is invariant under the $G$-symmetry transformation. (Readers who are not familiar with this statement can read the proof in Sec. IX.) Such a symmetry is an anomalous (or non-on-site) symmetry.

Since the action amplitude is not invariant locally, but invariant on the whole boundary $\partial M^{3}=M^{2}$, thus, under the symmetry transformation, the Lagrangian may change by a total derivative term:

$$
\mathcal{L}_{\mathrm{Bdry}, \partial M^{3}}[g g(x)]=\mathcal{L}_{\mathrm{Bdry}, \partial M^{3}}[g(x)]+\mathrm{d} \mathcal{L}^{\prime}[g(x)] .
$$

The presence of $\mathrm{d} \mathcal{L}^{\prime}[g(x)]$ is another sign of the anomalous symmetry. To understand the symmetry transformation on the boundary in more detail, we note that, in our case, $\mathrm{d} \mathcal{L}^{\prime}[g(x)]$ is given by

$$
\mathrm{e}^{-\int_{M^{2}} \mathrm{~d} \mathcal{L}^{\prime}[g(x)] d^{2} x}=\prod_{M^{2}} \frac{\nu_{3}^{s_{i j k}}\left(g_{i}, g_{j}, g_{k}, g^{-1} g^{*}\right)}{\nu_{3}^{S_{i j k}}\left(g_{i}, g_{j}, g_{k}, g^{*}\right)} .
$$

If we view

$$
f_{2}\left(g_{i}, g_{j}, g_{k}\right) \equiv \frac{\nu_{3}\left(g_{i}, g_{j}, g_{k}, g^{-1} g^{*}\right)}{\nu_{3}\left(g_{i}, g_{j}, g_{k}, g^{*}\right)},
$$

as a two-cochain, it is actually a two-coboundary (see Fig. 9):

$$
\begin{aligned}
f_{2}\left(g_{i}, g_{j}, g_{k}\right) & =\frac{\nu_{3}\left(g_{i}, g_{j}, g_{k}, g^{-1} g^{*}\right)}{\nu_{3}\left(g_{i}, g_{j}, g_{k}, g^{*}\right)} \\
& =\frac{\nu_{3}\left(g_{i}, g_{j}, g^{*}, g^{-1} g^{*}\right) \nu_{3}\left(g_{j}, g_{k}, g^{*}, g^{-1} g^{*}\right)}{\nu_{3}\left(g_{i}, g_{k}, g^{*}, g^{-1} g^{*}\right)} \\
& =\mathrm{d} f_{1},
\end{aligned}
$$

with a one-cochain $f_{1}$ as

$$
f_{1}\left(g_{i}, g_{j}\right)=\nu_{3}\left(g_{i}, g_{j}, g^{*}, g^{-1} g^{*}\right) .
$$

Thus,

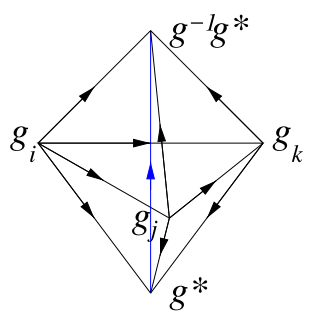

FIG. 9. Graphic representations of $f_{2}\left(g_{i}, g_{j}, g_{k}\right)=$ $\left[\nu_{3}\left(g_{i}, g_{j}, g_{k}, g^{-1} g^{*}\right) / \nu_{3}\left(g_{i}, g_{j}, g_{k}, g^{*}\right)\right]$, which is actually a coboundary. See Eq. (4.13).

$$
\mathrm{e}^{-\int_{M^{2}} \mathrm{~d} \mathcal{L}^{\prime}[g(x)] d^{2} x}=\prod_{M^{2}} f_{2}^{s_{i j k}}\left(g_{i}, g_{j}, g_{k}\right)=\prod_{\partial M^{2}} f_{1}^{s_{i j}}\left(g_{i}, g_{j}\right) .
$$

In some sense, $\mathcal{L}^{\prime}$ is given by $f_{1}$. When the spacetime boundary $M^{2}=\partial M^{3}$, we have $\partial M^{2}=\partial^{2} M^{3}=\varnothing$, and, therefore, Eq. (4.15) simplifies to

$$
\mathrm{e}^{-\int_{M^{2}} \mathrm{~d} \mathcal{L}^{\prime}[g(x)] d^{2} x}=1 .
$$

Thus, globally there is a global symmetry, as was claimed in Eq. (4.8), though it holds only up to a lattice version of a total derivative.

\section{Symmetry transformation on a spatial boundary in Hamiltonian formalism}

In the above, we have discussed the effective symmetry transformation on the spacetime boundary in Lagrangian formalism. Now, we will proceed with a Hamiltonian formalism.

What we mean by a Hamiltonian formalism is to choose a fixed space $M^{2}$, and use the path integral on $M^{2} \times I$ to construct the imaginary-time evolution unitary operator $\mathrm{e}^{-\hat{H}_{M^{2}}}$, where $I=[0,1]$ represents the time direction (see Fig. 10). The matrix elements of the imaginary-time evolution operator are $\left(\mathrm{e}^{-\hat{H}_{M^{2}}}\right)_{\left\{g_{i}^{\prime \prime}, \ldots\right\},\left\{g_{i}^{\prime}, \ldots\right\}}$, where $\left\{g_{i}^{\prime}, \ldots\right\}$ are the degrees of freedom on $M^{2} \times\{0\}$, and $\left\{g_{i}^{\prime \prime}, \ldots\right\}$ on $M^{2} \times\{1\}$. We may choose $M^{2} \times I$ to represent just one time step of evolution, so that there are no interior degrees of freedom to sum over. In this case, the unitary operator is

$$
\left(\mathrm{e}^{-\hat{H}_{M^{2}}}\right)_{\left\{g_{i}^{\prime \prime}, \ldots\right\},\left\{g_{i}^{\prime}, \ldots\right\}}=\prod_{M^{2} \times I} \nu_{3}^{s_{i j k l}}\left(g_{i}, g_{j}, g_{k}, g_{l}\right) .
$$

When the space $M^{2}$ has a boundary, then some degrees of freedom live on the boundary $\partial M^{2}$ and others live in the interior of $M^{2}$. We can ask about the properties of global symmetry transformations in two scenarios: The first is the symmetry of the whole bulk and the boundary included together, which is an on-site symmetry. The second is the symmetry of the effective boundary theory only, which turns out to be a non-on-site symmetry.

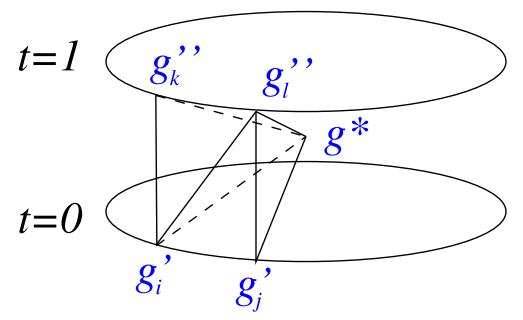

FIG. 10. $M^{2} \times I$ representing one step of imaginary time evolution, for the effective boundary theory. The space $M^{2}$ is given by the disk. 
(1) For the first scenario, the symmetry of the whole bulk and the boundary together, we have

$$
\left(\mathrm{e}^{-\hat{H}_{M^{2}}}\right)_{\left\{g g_{i}^{\prime \prime}, \ldots\right\},\left\{g g_{i}^{\prime}, \ldots\right\}}=\left(\mathrm{e}^{-\hat{H}_{M^{2}}}\right)_{\left\{g_{i}^{\prime \prime}, \ldots\right\},\left\{g_{i}^{\prime}, \ldots\right\}},
$$

because every homogeneous cochain satisfies $\nu_{3}\left(g g_{i}, g g_{j}, g g_{k}, g g_{l}\right)=\nu_{3}\left(g_{i}, g_{j}, g_{k}, g_{l}\right)$. If we write the evolution operator $\mathrm{e}^{-\hat{H}_{M^{2}}}$ explicitly, including the matrix elements and basis projectors, we see that

$$
\begin{aligned}
& \left|\left\{g g_{i}^{\prime \prime}, \ldots\right\}\right\rangle\left(\mathrm{e}^{-\hat{H}_{M_{2}}}\right)_{\left\{g g_{i}^{\prime \prime}, \ldots\right\},\left\{g g_{i}^{\prime}, \ldots\right\}}\left\langle\left\{g g_{i}^{\prime}, \ldots\right\}\right| \\
& =\hat{U}_{0}(g)\left|\left\{g_{i}^{\prime \prime}, \ldots\right\}\right\rangle\left(\mathrm{e}^{-\hat{H}_{M_{2}}}\right)_{\left\{g_{i}^{\prime \prime}, \ldots\right\},\left\{g_{i}^{\prime}, \ldots\right\}} \\
& \quad \times\left\langle\left\{g_{i}^{\prime}, \ldots\right\}\right| \hat{U}_{0}^{\dagger}(g),
\end{aligned}
$$

where $\hat{U}_{0}(g)$ generates the usual on-site $G$-symmetry transformation $\left|\left\{g_{i}, \ldots\right\}\right\rangle \rightarrow\left|\left\{g g_{i}, \ldots\right\}\right\rangle$. Thus, the $G$-symmetry transformation on the whole system (with bulk and boundary included) is an on-site symmetry, as it reasonably should be, as in condensed matter.

(2) For the second scenario, to obtain the symmetry of the effective boundary theory, we can simplify all the interior degrees of freedom into a single one $g^{*}$; then, the degrees of freedom on $M^{2}$ are given by $\left\{g_{1}, g_{2}, \ldots, g^{*}\right\}$, where $g_{i}$ live on the boundary $\partial M^{2}$ and $g^{*}$ lives in the interior of $M^{2}$ (see Fig. 10). Now, the imaginary-time evolution operator is given by

$$
\left(\mathrm{e}^{-\hat{H}_{\partial M^{2}}}\right)_{\left\{g_{i}^{\prime \prime}, \ldots\right\},\left\{g_{i}^{\prime}, \ldots\right\}}=\prod_{M^{2} \times I} \nu_{3}^{s_{i j k *}}\left(g_{i}, g_{j}, g_{k}, g^{*}\right),
$$

which defines an effective Hamiltonian for the boundary. Now, we are ready to ask: What is the symmetry of the effective boundary Hamiltonian, or effectively the symmetry of time evolution operator $\mathrm{e}^{-\hat{H}_{\partial M^{2}}}$ ?

The analysis of global symmetry in Sec. IV C 1 no longer applies. The discrete time evolution operator does not have the usual global symmetry:

$$
\left(\mathrm{e}^{-\hat{H}_{\partial M^{2}}}\right)_{\left\{g g_{i}^{\prime \prime}, \ldots\right\},\left\{g g_{i}^{\prime}, \ldots\right\}} \neq\left(\mathrm{e}^{-\hat{H}_{\partial M^{2}}}\right)_{\left\{g_{i}^{\prime \prime}, \ldots\right\},\left\{g_{i}^{\prime}, \ldots\right\}},
$$

since

$$
\begin{aligned}
& \prod_{M^{2} \times I} \nu_{3}^{s_{i j k *}}\left(g g_{i}, g g_{j}, g g_{k}, g^{*}\right) \\
& \quad=\prod_{M^{2} \times I} \nu_{3}^{s_{i j k *}}\left(g_{i}, g_{j}, g_{k}, g^{-1} g^{*}\right) \\
& \quad \neq \prod_{M^{2} \times I} \nu_{3}^{s_{i j k *}}\left(g_{i}, g_{j}, g_{k}, g^{*}\right) .
\end{aligned}
$$

The difference between two matrix elements $\left(\mathrm{e}^{-\hat{H}_{\partial M^{2}}}\right)_{\left\{g g_{i}^{\prime \prime}, \ldots\right\},\left\{g g_{i}^{\prime}, \ldots\right\}}$ and $\left(\mathrm{e}^{-\hat{H}_{\partial M^{2}}}\right)_{\left\{g_{i}^{\prime \prime}, \ldots\right\},\left\{g_{i}^{\prime}, \ldots\right\}}$ is just a $U(1)$ phase factor

$$
\begin{aligned}
\prod_{M^{2} \times I} & \frac{\nu_{3}^{s_{i j k}}\left(g_{i}, g_{j}, g_{k}, g^{-1} g^{*}\right)}{\nu_{3}^{s_{i j k}}\left(g_{i}, g_{j}, g_{k}, g^{*}\right)} \\
= & \prod_{\partial M^{2} \times I} f_{2}^{s_{i j k}}\left(g_{i}, g_{j}, g_{k}\right) \\
& =\prod_{\partial M^{2} \times \partial I} f_{1}^{s_{i j}}\left(g_{i}, g_{j}\right) \\
& =\prod_{\partial M^{2} \times \partial I} \nu_{3}^{s_{i j}}\left(g_{i}, g_{j}, g^{*}, g^{-1} g^{*}\right) \\
& =\frac{\prod_{(i j)} \nu_{3}^{s_{i j}}\left(g_{i}^{\prime \prime}, g_{j}^{\prime \prime}, g^{*}, g^{-1} g^{*}\right)}{\prod_{(i j)} \nu_{3}^{s_{i j}}\left(g_{i}^{\prime}, g_{j}^{\prime}, g^{*}, g^{-1} g^{*}\right)},
\end{aligned}
$$

where $\prod_{M^{2} \times I}$ multiplies over all the three-simplices in Fig. 10, $\prod_{\partial M^{2} \times I}$ over all the two-simplices on $\partial M^{2} \times I$, and $\prod_{\partial M^{2} \times \partial I}$ over all the one-simplices on the top and the bottom boundaries of $\partial M^{2} \times I$. Note that many oppositely oriented $\nu_{3}$ terms are canceled out in order to derive the last form of the above Eq. (4.21). This means that the boundary time evolution operator is invariant,

$$
\begin{aligned}
\left|\left\{g g_{i}^{\prime \prime}, \ldots\right\}\right\rangle\left(\mathrm{e}^{-\hat{H}_{\partial M^{2}}}\right)_{\left\{g g_{i}^{\prime \prime}, \ldots\right\},\left\{g g_{i}^{\prime}, \ldots\right\}}\left\langle\left\{g g_{i}^{\prime}, \ldots\right\}\right| \\
=\hat{U}(g)\left|\left\{g_{i}^{\prime \prime}, \ldots\right\}\right\rangle\left(\mathrm{e}^{-\hat{H}_{\partial M^{2}}}\right)_{\left\{g_{i}^{\prime \prime}, \ldots\right\},\left\{g_{i}^{\prime}, \ldots\right\}} \\
\quad \times\left\langle\left\{g_{i}^{\prime}, \ldots\right\}\right| \hat{U}^{\dagger}(g),
\end{aligned}
$$

under a modified $G$-symmetry transformation

$$
\hat{U}(g) \equiv \hat{U}_{0}(g) U_{\left\{g_{i}, \ldots\right\}},
$$

where

$$
U_{\left\{g_{i}, \ldots\right\}}=\prod_{(i j)} \nu_{3}^{s_{i j}}\left(g_{i}, g_{j}, g^{*}, g^{-1} g^{*}\right)
$$

and $\hat{U}_{0}(g)$ generates the usual on-site $G$-symmetry transformation $\left|\left\{g_{i}, \ldots\right\}\right\rangle \rightarrow\left|\left\{g g_{i}, \ldots\right\}\right\rangle$. The phase factor $U_{\left\{g_{i}, \ldots\right\}}$ makes the $G$-symmetry non-on-site at the boundary.

We have written these formulas in $2+1$ dimensions, but they all can be generalized. In $d$ dimensions, we have an effect boundary symmetry operator $\hat{U}(g)$ acting on $\partial M^{d-1}$ for the effective boundary Hamiltonian $\mathrm{e}^{-\hat{H}_{\partial M^{d-1}}}$ :

$$
\begin{aligned}
\hat{U}(g) & \equiv \hat{U}_{0}(g) U_{\left\{g_{i}, \ldots\right\}} \\
& =\hat{U}_{0}(g) \prod_{(i j \cdots \ell) \in \partial M^{d-1}} \nu_{d}^{s_{i j \cdots \ell}}\left(g_{i}, g_{j}, \ldots, g_{\ell}, g^{*}, g^{-1} g^{*}\right) .
\end{aligned}
$$




\section{The second boundary of a generic SPT state: Gapped boundary by extending the $G$-symmetry to an $\boldsymbol{H}$-symmetry}

In Sec. III A and also in Sec. IV B, we considered the path integral of a $G$-SPT state described by a homogeneous cocycle $\nu_{d} \in \mathcal{H}^{d}[G, U(1)]$. The path integral that we studied in that section remained $G$-symmetry invariant even on a manifold with a boundary, where the $G$-symmetry is an onsite symmetry in the bulk. However, if we integrate out the bulk degrees of freedom, the effective boundary theory will have an effective $G$-symmetry, which must be non-on-site (i.e., anomalous) on the boundary. This anomalous $G$ symmetry on the boundary forces the boundary to have some nontrivial dynamical properties.

However, the simple model introduced in Sec. IV B has a complicated boundary dynamics, which is hard to solve. There are several standard ways to modify the construction in Sec. IV B to get a boundary that can be solved exactly. One way to do so is to constrain the group variables $g_{i}$ on boundary sites to all equal 1 , or at least to take values in a subgroup $G^{\prime} \subseteq G$ such that the cohomology class of $\nu_{d}$ becomes trivial when restricted to $G^{\prime}$. Given this, after possibly modifying $\nu_{d}$ by a coboundary, we can assume that $\nu_{d}=1$ when the group variables $g_{i}$ all belong to $G^{\prime}$. In this case, the action amplitudes for the boundary effective theory Eq. (4.7) are always equal to 1 (after choosing $g^{*} \in G^{\prime}$ ). So, the boundary constructed in this way is exactly soluble and is gapped. This construction amounts to spontaneous or explicit breaking of the symmetry from $G$ to $G^{\prime}$.

In this section, we will explain another procedure to construct a model with the same bulk physics and an exactly soluble gapped boundary. This will be accomplished by extending (rather than breaking) the global symmetry along the boundary. Then, as in our explicit example of the CZX model in Sec. III B, we get a boundary state that is gapped and symmetric, but the symmetry along the boundary is enhanced relative to the bulk.

\section{A purely mathematical setup on that $G$-cocycle is trivialized in $\boldsymbol{H}$}

To describe the symmetry-extended boundary, let us introduce a purely mathematical result. We consider an extension of $G$,

$$
1 \rightarrow K \rightarrow H \stackrel{r}{\rightarrow} G \rightarrow 1,
$$

where $K$ is a normal subgroup of $H$, and $H / K=G$. Here, $r$ is a surjective group homomorphism from $H$ to $G$. A " $G$-variable" $G$-cocycle $\nu_{d}\left(g_{0}, \ldots, g_{d}\right)$ can be "pulled back" to an " $H$-variable" $H$-cocycle $\nu_{d}^{H}\left(h_{0}, \ldots, h_{d}\right)$, defined by

$\nu_{d}^{H}\left(h_{0}, \ldots, h_{d}\right)=\nu_{d}\left[r\left(h_{0}\right), \ldots, r\left(h_{d}\right)\right] \equiv \nu_{d}^{G}\left[r\left(h_{0}\right), \ldots, r\left(h_{d}\right)\right]$.
The case of interest to us is that $\nu_{d}^{H}$ is trivial in $\mathcal{H}^{d}[H, U(1)]$. This means $\nu_{d}^{H}\left(h_{0}, \ldots, h_{d}\right)$ can be rewritten as a coboundary, namely,

$$
\begin{aligned}
\nu_{d}^{H}\left(h_{0}, \ldots, h_{d}\right) & =\delta \mu_{d-1}^{H}\left(h_{0}, \ldots, h_{d}\right) \\
& \equiv \frac{\prod_{i=\mathrm{even}} \mu_{d-1}^{H}\left(h_{0}, \ldots, \hat{h}_{i}, \ldots, h_{d}\right)}{\prod_{i=\mathrm{odd}} \mu_{d-1}^{H}\left(h_{0}, \ldots, \hat{h}_{i}, \ldots, h_{d}\right)} .
\end{aligned}
$$

(The symbol $\hat{h}_{i}$ is an instruction to omit $h_{i}$ from the sequence.)

For the convenience and the preciseness of the notation, we can also shorten the above Eq. (4.27) to

$$
\nu_{d}^{G}[r(h)]=\nu_{d}^{H}(h)=\delta \mu_{d-1}^{H}(h),
$$

where the variable $h$ in the bracket is a shorthand of many copies of group elements in a direct product group of $H$. By pulling back a $G$-cocycle $\nu_{d}^{G}$ back to $H$, it becomes an $H$-coboundary $\delta \mu_{d-1}^{H}$. Formally, we mean that a nontrivial $G$-cocycle

$$
\nu_{d}^{G} \in \mathcal{H}^{d}[G, U(1)]
$$

becomes a trivial element when it is pulled back (denoted as $*$ ) to $H$

$$
r^{*} \nu_{d}^{G}=\nu_{d}^{H}=\delta \mu_{d-1}^{H} \in \mathcal{H}^{d}[H, U(1)] .
$$

Saying that this element is trivial means that the corresponding cocycle is a coboundary.

Here, $\mu_{d-1}^{H}\left(h_{0}, \ldots, h_{d-1}\right)$ is a homogeneous $(d-1)$ cochain:

$$
\mu_{d-1}^{H}\left(h h_{0}, \ldots, h h_{d-1}\right)=\mu_{d-1}^{H}\left(h_{0}, \ldots, h_{d-1}\right) .
$$

The definition of $\nu_{d}^{H}$ also ensures that

$\nu_{d}^{H}\left(v_{0} h_{0}, \ldots, v_{d} h_{d}\right)=\nu_{d}^{H}\left(h_{0}, \ldots, h_{d}\right), \quad v_{i} \in K$,

since $r\left(v_{i}\right)=1$ is trivial in $G$ for any $v_{i} \in K$. In particular, $\nu_{d}^{H}\left(v_{0}, \ldots, v_{d}\right)=1, v_{i} \in K$, and therefore,

$$
\frac{\prod_{i=\mathrm{even}} \mu_{d-1}^{H}\left(v_{0}, \ldots, \hat{v}_{i}, \ldots, v_{d}\right)}{\prod_{i=\mathrm{odd}} \mu_{d-1}^{H}\left(v_{0}, \ldots, \hat{v}_{i}, \ldots, v_{d}\right)}=1 .
$$

Thus, when we restrict to $K$, the cochain $\mu_{d-1}^{H}\left(v_{0}, \ldots, v_{d-1}\right)$ becomes a cocycle $\mu_{d-1}^{K}$ in $\mathcal{H}^{d-1}[K, U(1)]$. An important detail is that, in general, the cohomology class of $\mu_{d-1}^{K}$ is not uniquely determined by the original cocycle $\nu_{d}$. In general, it can depend on the choice of cochain $\mu_{d-1}^{H}$ that was used to trivialize $\nu_{d}^{H}$.

In fact, let $\mu_{d-1}^{H}$ and $\tilde{\mu}_{d-1}^{H}$ be two cochains, either of which could be used to trivialize $\nu_{d}^{H}$ : 


$$
\nu_{d}^{H}=\delta \mu_{d-1}^{H}=\delta \tilde{\mu}_{d-1}^{H} .
$$

Then, $\nu_{d-1}^{H}=\mu_{d-1}^{H}\left(\tilde{\mu}_{d-1}^{H}\right)^{-1}$ is a cocycle, $\delta \nu_{d-1}^{H}=1$. So, $\nu_{d-1}^{H}$ has a class in $\mathcal{H}^{d-1}[H, U(1)]$. If this class is nontrivial, the gapped boundary states that we will construct using $\mu_{d-1}^{H}$ and $\tilde{\mu}_{d-1}^{H}$ are inequivalent. Thus, the number of inequivalent gapped boundary states that we can make by the construction described below (keeping fixed $H$ and $K)$ is the order of the finite group $\mathcal{H}^{d-1}[H, U(1)]$. [60]

A nontrivial class in $\mathcal{H}^{d-1}[H, U(1)]$ may or may not remain nontrivial after restriction from $H$ to $K$, so, in general, as stated above, the cohomology class of $\nu_{d-1}^{K}$ can depend on the choice of $\mu_{d-1}^{H}$.

\section{H-symmetry extended boundary: By extending $\boldsymbol{G}$-symmetry to $\boldsymbol{H}$-symmetry}

To construct the second boundary of a generic SPT state, we allow the degrees of freedom on the vertices at the boundary to be labeled by $h_{i} \in H$. This amounts to adding new degrees of freedom along the boundary. The degrees of freedom on the vertices in the bulk are still labeled by $g_{i} \in G$. With this enhancement of the boundary variables, we can write down the action amplitude for the second construction as

$e^{-\int_{M^{d}} \mathcal{L}_{\mathrm{Bulk}} d^{d} x}=\frac{\prod_{M^{d} \nu_{d}^{s_{01 \cdots d}}}\left(g_{0}, g_{1}, \ldots, g_{d}\right)}{\prod_{\partial M^{d}}\left(\mu_{d-1}^{H}\right)^{s_{01 \ldots(d-1)}}\left(h_{0}, h_{1}, \ldots, h_{d-1}\right)}$,

where $\nu_{d}$ and $\mu_{d-1}^{H}$ are the cochains introduced in the last section and $M^{d}$ may have a boundary. Here, if a vertex in $\nu_{d}\left(g_{0}, g_{1}, \ldots, g_{d}\right)$ is on the boundary, the corresponding $g_{i}$ is given by $g_{i}=r\left(h_{i}\right)$.

We note that, since $r: H \rightarrow G$ is a group homomorphism, the action $h: H \rightarrow H, h_{i} \rightarrow h h_{i}$, induces an action $r(h)$ : $G \rightarrow G, g_{i} \rightarrow r(h) g_{i}$. Therefore, the total action amplitude Eq. (4.35) has $H$-symmetry:

$$
\begin{aligned}
& \frac{\prod_{M^{d} \nu_{d}^{s_{01 \cdots d}}\left(g_{0}, g_{1}, \ldots, g_{d}\right)}}{\prod_{\partial M^{d}}\left(\mu_{d-1}^{H}\right)^{s_{01 \ldots(d-1)}}\left(h_{0}, h_{1}, \ldots, h_{d-1}\right)}
\end{aligned}
$$

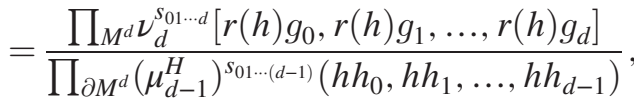

where $h \in H$. In the bulk, the symmetry is $G$, but along the boundary, it is extended to $H$. Such a total action amplitude defines our second construction of the boundary of a $G$-SPT state, which has a symmetry extension $G$ lifted to $H$ on the boundary. We return to more details on this model in Sec. IX.

The bulk of the constructed model is described by the same group cocycle $\nu_{d}$, which gives rise to the $G$-SPT state. But the boundary has an extended symmetry $H$. In this case, we should view the whole system (bulk and boundary) as having an extended $H$-symmetry, with the $K$ subgroup acting trivially in the bulk. So the effective symmetry in the bulk is $G=H / K$.

The dynamics of our second boundary is very simple, since the total action amplitude Eq. (4.35) is always equal to 1 by construction:

$$
\begin{aligned}
\prod_{M^{d}} \nu_{d}^{s_{01 \cdots d}}\left(g_{0}, g_{1}, \ldots, g_{d}\right) & =\prod_{M^{d}}\left(\nu_{d}^{H}\right)^{s_{01 \cdots d}}\left(h_{0}, h_{1}, \ldots, h_{d}\right) \\
& =\prod_{\partial M^{d}}\left(\mu_{d-1}^{H}\right)^{s_{01 \cdots(d-1)}}\left(h_{0}, h_{1}, \ldots, h_{d-1}\right),
\end{aligned}
$$

where $g_{i}=r\left(h_{i}\right)$. Thus, the ground state is always gapped and there is no ground state degeneracy regardless of whether the system has a boundary or not. In other words, the second boundary of the $G$-SPT state is gapped with $H$-symmetry and no topological order. The gapped boundary with $H$-symmetry and no topological order is possible, since we have chosen $H$ so that when we view the $G$-SPT state as an $H$-SPT state, the nontrivial $G$-SPT state becomes a trivial $H$-SPT state.

\section{E. On-site (anomaly-free) $\boldsymbol{H}$-symmetry transformation on the boundary effective theory}

Now, we show that symmetry extension, as described in Sec. IV D 2, gives a boundary state with on-site (anomalyfree) $H$-symmetry, based on the Hamiltonian formalism on the boundary. This section directly parallels the previous discussion in Sec. IV C, where a nontrivial $G$-cocycle gives rise to a non-on-site effective $G$-symmetry on the boundary. After extending the symmetry to $H$, the nontrivial $G$-cocycle $\nu_{d}$ becomes a trivial $H$-cocycle $\nu_{d}^{H}$, which in turn gives rise to an on-site effective $H$-symmetry for the boundary effective theory.

Taking $d=3$ as an example, Eqs. (4.13), (4.14), and (4.15) of Sec. IV C 1 still hold. Furthermore, when $h_{i}, h_{j}$, and $h_{k}$ are boundary degrees of freedom in $H$, Eq. (4.13) becomes

$$
f_{2}\left(h_{i}, h_{j}, h_{k}\right)=\frac{\mu_{2}^{H}\left(h_{i}, h_{j}, h^{-1} h^{*}\right) \mu_{2}^{H}\left(h_{j}, h_{k}, h^{-1} h^{*}\right) \mu_{2}^{H}\left(h_{i}, h_{k}, h^{-1} h^{*}\right)^{-1}}{\mu_{2}^{H}\left(h_{i}, h_{j}, h^{*}\right) \mu_{2}^{H}\left(h_{j}, h_{k}, h^{*}\right) \mu_{2}^{H}\left(h_{i}, h_{k}, h^{*}\right)^{-1}}=\mathrm{d} f_{1} .
$$




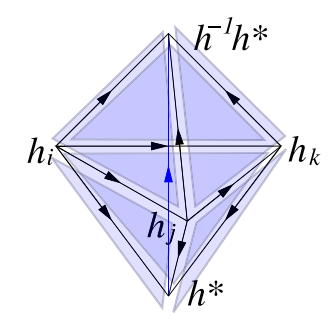

FIG. 11. Graphic representation of $f_{2}\left(h_{i}, h_{j}, h_{k}\right)=$ $\left[\mu_{2}^{H}\left(h_{i}, h_{j}, h^{-1} h^{*}\right) / \mu_{2}^{H}\left(h_{i}, h_{j}, h^{*}\right)\right]\left[\mu_{2}^{H}\left(h_{j}, h_{k}, h^{-1} h^{*}\right) / \mu_{2}^{H}\left(h_{j}, h_{k}, h^{*}\right)\right]$ $\left[\mu_{2}^{H}\left(h_{i}, h_{k}, h^{*}\right) / \mu_{2}^{H}\left(h_{i}, h_{k}, h^{-1} h^{*}\right)\right]=\mathrm{d} f_{1}$, again as a coboundary. Each shaded blue triangle is assigned with a split cochain $\mu_{2}^{H}$. See Eqs. (4.38) and (4.39).

See Fig. 11 for an illustration. Here, $\mu_{2}^{H}$ is a homogeneous two-cochain that splits $\nu_{3}^{H}$ [or $\nu_{3}^{G}(\{r(h)\})$ ] and satisfies $\mu_{2}^{H}\left(h_{i}, h_{j}, h^{-1} h^{*}\right)=\mu_{2}^{H}\left(h h_{i}, h h_{j}, h^{*}\right)$. Now, the split twocochain $f_{1}$ in Eq. (4.14) has a new form:

$$
f_{1}\left(h_{i}, h_{j}\right)=\frac{\mu_{2}^{H}\left(h_{i}, h_{j}, h^{-1} h^{*}\right)}{\mu_{2}^{H}\left(h_{i}, h_{j}, h^{*}\right)} .
$$

To show more clearly that $H$-symmetry can be made onsite and anomaly-free in any dimension $d$, we note that the action amplitude Eq. (4.35) can be rewritten as

$$
e^{-\int_{M^{d}} \mathcal{L}_{\text {Bulk }} d^{d} x}=\frac{\prod_{M^{d}}\left(\nu_{d}^{H}\right)^{s_{01 \cdots d}}\left(h_{0}, h_{1}, \ldots, h_{d}\right)}{\prod_{\partial M^{d}}\left(\mu_{d-1}^{H}\right)^{s_{01 \cdots(d-1)}}\left(h_{0}, h_{1}, \ldots, h_{d-1}\right)} .
$$

Each local term $\left(\mu_{d-1}^{H}\right)^{s_{01 \cdots(d-1)}}\left(h_{0}, h_{1}, \ldots, h_{d-1}\right)$ is already invariant under $H$-symmetry transformation on the boundary. So, we will drop it. The term $\left(\nu_{d}^{H}\right)^{s_{01 \cdots d}}\left(h_{0}, h_{1}, \ldots, h_{d}\right)$ may not be invariant under $H$-symmetry transformation on the boundary, although their product $\prod_{M^{d}}\left(\nu_{d}^{H}\right)^{s_{01 \cdots d}}\left(h_{0}, h_{1}, \ldots, h_{d}\right)$ is. This may lead to a non-on-site $H$-symmetry. Repeating the calculation in Sec. IVC, we found that the discrete time evolution operator $\mathrm{e}^{-\hat{H}_{\partial M^{d-1}}}$ does not have the usual global symmetry, where their matrix elements follow:

$$
\left(\mathrm{e}^{-\hat{H}_{\partial M^{d-1}}}\right)_{\left\{h h_{i}^{\prime \prime}, \ldots\right\},\left\{h h_{i}^{\prime}, \ldots\right\}} \neq\left(\mathrm{e}^{-\hat{H}_{\partial M^{d-1}}}\right)_{\left\{h_{i}^{\prime \prime}, \ldots\right\},\left\{h_{i}^{\prime}, \ldots\right\}} \cdot
$$

But, it is invariant

$$
\begin{aligned}
& \left|\left\{h h_{i}^{\prime \prime}, \ldots\right\}\right\rangle\left(\mathrm{e}^{-\hat{H}_{\partial M^{d-1}}}\right)_{\left\{h h_{i}^{\prime \prime}, \ldots\right\},\left\{h h_{i}^{\prime}, \ldots\right\}}\left\langle\left\{h h_{i}^{\prime}, \ldots\right\}\right| \\
& \quad=\hat{U}(h)\left|\left\{h_{i}^{\prime \prime}, \ldots\right\}\right\rangle\left(\mathrm{e}^{-\hat{H}_{\partial M^{d-1}}}\right)_{\left\{h_{i}^{\prime \prime}, \ldots\right\},\left\{h_{i}^{\prime}, \ldots\right\}}\left\langle\left\{h_{i}^{\prime}, \ldots\right\}\right| \hat{U}^{\dagger}(h),
\end{aligned}
$$

under a modified symmetry transformation operator

$$
\hat{U}(h) \equiv \hat{U}_{0}(h) \prod_{(i j \ldots \ell) \in \partial M^{d-1}}\left(\nu_{d}^{H}\right)^{s_{i j} \cdots}\left(h_{i}, h_{j}, \ldots, h_{\ell}, h^{*}, h^{-1} h^{*}\right),
$$

which appears to be non-on-site. However, since $\nu_{d}^{H}=$ $\delta \mu_{d-1}^{H}$ is a coboundary, the above can be rewritten as (see Figs. 10 and 12)

$\hat{U}(h)=\hat{U}_{0}(h) \frac{\prod_{(i j \cdots \ell) \in \partial M^{d-1}}\left(\mu_{d-1}^{H}\right)^{s_{i j \cdots \ell}}\left(h h_{i}, h h_{j}, \ldots, h h_{\ell}, h^{*}\right)}{\prod_{(i j \cdots \ell) \in \partial M^{d-1}}\left(\mu_{d-1}^{H}\right)^{s_{i j \cdots \ell}}\left(h_{i}, h_{j}, \ldots, h_{\ell}, h^{*}\right)}$.

After a local unitary transformation $\quad\left|\left\{h_{i}\right\}\right\rangle \rightarrow$ $W\left(\left\{h_{i}\right\}\right)\left|\left\{h_{i}\right\}\right\rangle \equiv\left|\left\{h_{i}\right\}^{\prime}\right\rangle$, with

$$
W\left(\left\{h_{i}\right\}\right) \equiv \prod_{(i j \ldots) \in \partial M^{d-1}} \mu_{d-1}^{H}\left(h_{i}, h_{j}, \ldots, h^{*}\right),
$$

we can change the above $H$-symmetry transformation to

$$
\hat{U}(h) \rightarrow W^{\dagger} \hat{U}(h) W=\hat{U}_{0}(h),
$$

which indeed becomes on-site. The on-site symmetry $\hat{U}_{0}(h)$ makes the time evolution operator invariant under

$$
\begin{aligned}
\left|\left\{h h_{i}^{\prime \prime}, \ldots\right\}^{\prime}\right\rangle\left(\mathrm{e}^{-\hat{H}_{\partial M^{d-1}}}\right)_{\left\{h h_{i}^{\prime \prime}, \ldots\right\},\left\{h h_{i}^{\prime}, \ldots\right\}}\left\langle\left\{h h_{i}^{\prime}, \ldots\right\}^{\prime}\right| \\
=\hat{U}_{0}(h)\left|\left\{h_{i}^{\prime \prime}, \ldots\right\}^{\prime}\right\rangle\left(\mathrm{e}^{-\hat{H}_{\partial M^{d-1}}}\right)_{\left\{h_{i}^{\prime \prime}, \ldots\right\},\left\{h_{i}^{\prime}, \ldots\right\}} \\
\quad \times\left\langle\left\{h_{i}^{\prime}, \ldots\right\}^{\prime}\right| \hat{U}_{0}^{\dagger}(h) .
\end{aligned}
$$

The subtle difference between Secs. IV C and IV E is that the $\nu_{d}\left(g_{i}, g_{j}, \ldots, g_{\ell}, g^{*}, g^{-1} g^{*}\right)$ cannot be absorbed through local unitary transformations, but its split form $\mu_{d-1}^{H}\left(h_{i}, h_{j}, \ldots, h^{*}\right)$ can be absorbed. Namely, one can think of $\mu_{d-1}^{H}$ as an output of a local unitary matrix acting on local nearby sites with input data $h_{i}, h_{j}, \ldots$ in a quantum circuit.

To summarize what we did in Secs. IV C and IV E, the $G$-symmetry transformation on the boundary was non-onsite and, thus, anomalous. The $H$-symmetry transformation on the boundary is now made to be on-site, by pulling back $G$ to $H$; thus, it is anomaly free in $H$.

\section{F. The third boundary of a generic SPT state: A gapped symmetric boundary that violates locality with (hard) gauge fields}

In the last section, we constructed a gapped symmetric boundary of a SPT state such that the global symmetry is extended from $G$ to $H$ along the boundary. Such boundary enhancement of the symmetry is usually [61] not natural in condensed matter physics. Just as in our discussion of the CZX model in Secs. III C and III D, the way to avoid symmetry extension is to gauge the boundary symmetry $K$, 


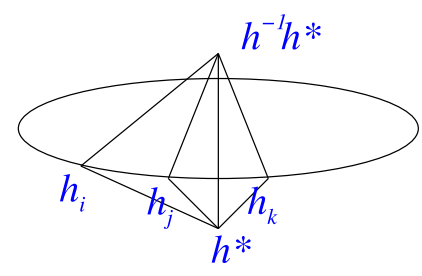

FIG. 12. Geometric picture to explain the calculation from Eq. (4.42) to Eq. (4.43) (for the $d=3$ case). $\prod_{(i j \cdots \ell) \in \partial M^{d-1}}\left(\nu_{d}^{H}\right)^{s_{i j \cdots \ell}}\left(h_{i}, h_{j}, \ldots, h_{\ell}, h^{*}, h^{-1} h^{*}\right)$ in Eq. (4.42) is a product over all the three-simplices in the figure. $\prod_{(i j \cdots \ell) \in \partial M^{d-1}}\left(\mu_{d-1}^{H}\right)^{s_{i j \cdots \ell}}\left(h h_{i}, h h_{j}, \ldots, h h_{\ell}, h^{*}\right)=\prod_{(i j \cdots \ell) \in \partial M^{d-1} \times}$ $\left(\mu_{d-1}^{H}\right)^{s_{i j \cdots} \cdots}\left(h_{i}, h_{j}, \ldots, h_{\ell}, h^{-1} h^{*}\right)$ is a product over all the twosimplices on the top surface, and $\prod_{(i j \cdots \ell) \in \partial M^{d-1}}\left(\mu_{d-1}^{H}\right)^{s_{i j \cdots \ell}} \times$ $\left(h_{i}, h_{j}, \ldots, h_{\ell}, h^{*}\right)$ is a product over all the two-simplices on the bottom surface.

giving a construction in which the full global symmetry group is $G$ (or $G^{\prime}$ in the more general mixed breaking and extension construction described in Sec. VIII B).

As in the CZX model, there are broadly two approaches to gauging the $K$-symmetry. One may use "hard gauging," in which one introduces (on the boundary) elementary fields that gauge the $K$-symmetry, or "soft gauging," in which the boundary gauge fields are emergent. Hard gauging is generally a little quicker to describe, so we begin with it, but soft gauging, which will be the topic of Sec. IV G, is more natural in condensed matter physics because it can be strictly local or "on-site." Our discussion here and in the next section is roughly parallel to Secs. III C and III D on the CZX model.

To construct a new boundary, let us consider a system on a $d$-dimensional space-time manifold $M^{d}$, with a triangulation that has a branching structure. A vertex $i$ inside $M^{d}$ carries a degree of freedom $g_{i} \in G$. A vertex $i$ on the boundary $\partial M^{d}$ carries a degree of freedom $h_{i} \in H$. A link $(i j)$ on the boundary $\partial M^{d}$ carries a degree of freedom $v_{i j} \in K$. See Fig. 13 .

We choose the action amplitude of our new model to be

$$
\begin{aligned}
\mathrm{e}^{-\int_{M^{d}} \mathcal{L} d^{d} x}= & \prod_{\left(i_{0} \cdots i_{d}\right) \in M^{d}} \nu_{d}^{s_{i_{0} \cdots i_{d}}}\left(g_{i_{0}}, \ldots, g_{i_{d}}\right) \\
& \times \prod_{\left(i_{0} \cdots i_{d-1}\right) \in \partial M^{d}}\left(\mathcal{V}_{d-1}^{H, K}\right)^{-s_{i_{0}} \cdots i_{d-1}} \\
& \times\left(h_{i_{0}}, \ldots, h_{i_{d-1}} ; v_{i_{0} i_{1}}, v_{i_{0} i_{2}}, \ldots\right),
\end{aligned}
$$

where $\prod_{\left(i_{0} \cdots i_{d}\right)}$ is a product over $d$-dimensional simplices $\left(i_{0} \cdots i_{d}\right)$ in the bulk, and $\prod_{\left(i_{0} \cdots i_{d-1}\right)}$ is a product over $(d-1)$-dimensional simplices $\left(i_{0} \cdots i_{d-1}\right)$ on the boundary. $s_{i_{0} \cdots i_{d}}= \pm 1$ is the orientation of the $d$-simplex $\left(i_{0} \cdots i_{d}\right)$, and $s_{i_{0} \cdots i_{d-1}}= \pm 1$ is the orientation of the $(d-1)$-simplex $\left(i_{0} \cdots i_{d-1}\right)$. Finally, $\mathcal{V}_{d-1}^{H, K}$ will be defined

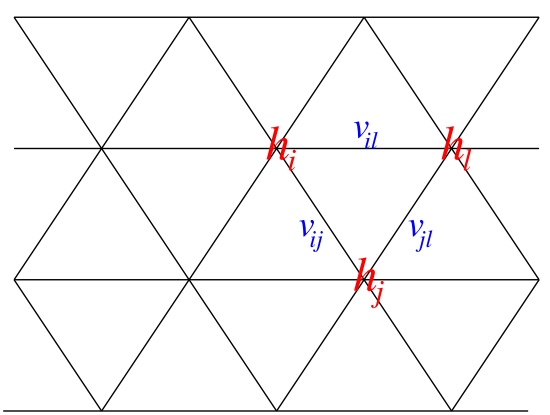

FIG. 13. A boundary of a $G$-SPT state. A vertex $i$ on the boundary carries $h_{i} \in H$, and a link $(i j)$ carries $v_{i j} \in K$.

in Sec. IV F 1, using $\mu_{d-1}^{H}$ introduced in Sec. IV D 1, as well as "hard-gauge fields" $v_{i j}$ along boundary links.

In the action amplitude Eq. (4.45), $\nu_{d} \in \mathcal{H}^{d}[G, U(1)]$ is the cocycle describing the $G$-SPT state. We have assumed that if a vertex $i$ in $\nu_{d}\left(g_{0}, \ldots, g_{d}\right)$ is on the boundary, then the corresponding $g_{i}$ is given by $g_{i}=r\left(h_{i}\right)$.

\section{A cochain that encodes "hard-gauge fields"}

The generalized cochain $\mathcal{V}_{d-1}^{H, K}\left(h_{i_{0}}, \ldots, h_{i_{d-1}} ; v_{i_{0} i_{1}}\right.$, $\left.v_{i_{0} i_{2}}, \ldots\right)$ will be defined for boundary simplices. It will depend on $H$-valued boundary spins $h_{i}$, as well as $K$-valued boundary link variables $v_{i j}$. As usual in lattice gauge theory, we can regard $v_{i j}$ as a $K$-gauge connection on the link $i j$.

First, we assume that $\mathcal{V}_{d-1}^{H, K}\left(h_{i_{0}}, \ldots, h_{i_{d-1}} ; v_{i_{0} i_{1}}, v_{i_{0} i_{2}}, \ldots\right)=0$ for any configurations $v_{i j}$ that do not satisfy $v_{i_{1} i_{2}} v_{i_{2} i_{3}}=v_{i_{1} i_{3}}$, for some $i_{1}, i_{2}, i_{3}$. So, only the $v_{i j}$ configurations that satisfy

$$
v_{i_{1} i_{2}} v_{i_{2} i_{3}}=v_{i_{1} i_{3}}
$$

on every triangle can contribute to the path integral. This means that only flat $K$-gauge fields are allowed.

For a flat connection on a simplex with vertices $i_{0}, \ldots, i_{d-1}$, all of the $v_{i_{j} i_{k}}$ can be expressed in terms of $v_{01}, v_{12}, v_{23}, \ldots, v_{d-2, d-1}$. So likewise, $\mathcal{V}_{d-1}^{H, K}\left(h_{0}, \ldots, h_{d-1}\right.$; $\left.v_{01}, v_{02}, v_{12}, \ldots\right)$ can be expressed as $\mathcal{V}_{d-1}^{H, K}\left(h_{0}, \ldots, h_{d-1}\right.$; $\left.v_{01}, v_{12}, \ldots, v_{d-2, d-1}\right)$. We define $\mathcal{V}_{d-1}^{H, K}$ in terms of the homogeneous cochain $\mu_{d-1}^{H}$ of Sec. IV D 1 by

$$
\begin{aligned}
& \mathcal{V}_{d-1}^{H, K}\left(h_{0}, \ldots, h_{d-1} ; v_{01}, v_{02}, v_{12}, \ldots\right) \\
& \quad=\mathcal{V}_{d-1}^{H, K}\left(h_{0}, \ldots, h_{d-1} ; v_{01}, v_{12}, \ldots, v_{d-2, d-1}\right) \\
& \quad=\mu_{d-1}^{H}\left(h_{0}, v_{01} h_{1}, v_{01} v_{12} h_{2}, \ldots\right) .
\end{aligned}
$$

In other words,

$$
\begin{aligned}
& \mathcal{V}_{d-1}^{H, K}\left(h_{0}, \ldots, h_{d-1} ; v_{01}, v_{12}, \ldots, v_{d-2, d-1}\right) \\
& \quad=\mu_{d-1}^{H}\left(\tilde{h}_{0}, \tilde{h}_{1}, \tilde{h}_{2}, \ldots\right),
\end{aligned}
$$


where $\tilde{h}_{i}$ is given by $h_{i}$ parallel transported from site $i$ to site 0 using the connection $v_{i j}$ :

$$
\tilde{h}_{i}=v_{01} v_{12} \ldots v_{i-1, i} h_{i} .
$$

We note that $\mathcal{V}_{d-1}^{H, K}$ has a local $K$-symmetry generated by $v_{0}, v_{1}, \ldots \in K$ :

$$
\begin{aligned}
& \mathcal{V}_{d-1}^{H, K}\left(v_{0} h_{0}, \ldots, v_{d-1} h_{d-1} ; v_{01}, v_{12}, \ldots, v_{d-2, d-1}\right) \\
& \quad=\mathcal{V}_{d-1}^{H, K}\left(h_{0}, \ldots, h_{d-1} ; v_{0}^{-1} v_{01} v_{1}, v_{1}^{-1} v_{12} v_{2}, \ldots\right) .
\end{aligned}
$$

Next, we will view such a boundary local symmetry as a $K$-gauge redundancy by viewing two boundary configurations $\left(h_{i}, v_{i j}\right)$ and $\left(h_{i}^{\prime}, v_{i j}^{\prime}\right)$ as the same configuration if they are related by a gauge transformation:

$$
h_{i}^{\prime}=v_{i} h_{i}, \quad v_{i j}^{\prime}=v_{i} v_{i j} v_{j}^{-1}, \quad v_{i} \in K .
$$

Equation (4.50) ensures the gauge invariance of the boundary action.

Now that we have gauged the $K$-symmetry, the global symmetry of the full system, including its boundary, is $G$. However, viewing two boundary configurations $\left(h_{i}, v_{i j}\right)$ and $\left(h_{i}^{\prime}, v_{i j}^{\prime}\right)$ as the same configuration makes the gauged theory no longer a local bosonic system. This is because the number of different (i.e., gauge inequivalent) configurations on the space-time boundary $\partial M^{d}$ is given by [62]

$$
\frac{|H|^{N_{v}}|K|^{N_{l}}}{|K|^{N_{v}}}|K|^{\left|\pi_{0}\left(\partial M^{d}\right)\right|},
$$

where $N_{v}$ is the number of vertices, $N_{l}$ is the number of links on the boundary $\partial M^{d}$, and $\mid$ Set $\mid$ is the number of elements in the Set. Here, we count all the distinct configurations of vertex variables of $H$ and link variables of $K$, identifying them up to $K$-gauge transformations on the vertices. We consider all higher energetic configurations, which include both flat and locally nonflat configurations, much more than just ground-state sectors. Constant gauge transformations yield an additional factor $|K|^{\left|\pi_{0}\left(\partial M^{d}\right)\right|}$. The appearance of the factor $|K|^{\left|\pi_{0}\left(\partial M^{d}\right)\right|}$, whose exponent is not linear in $N_{v}$ and $N_{l}$, implies a nonlocal system. So, the third boundary is no longer local in that strict sense. In Sec. IV F 2, we show that this nonlocal boundary is gapped and symmetric. In Sec. IV G, we will replace hard gauging with soft gauging and thereby get a boundary that is fully local and on-site, while still gapped and symmetric.

\section{A model that violates the locality for the boundary theory}

In the path integral, we only sum over gauge distinct configurations:

$$
\begin{aligned}
Z= & \sum_{\left\{g_{i},\left[h_{i}, h_{i j}\right]\right\}} \prod_{\left(i_{0} \cdots i_{d}\right) \in M^{d}} \nu_{d}^{s_{i_{0} \cdots i_{d}}}\left(g_{i_{0}}, \ldots, g_{i_{d}}\right) \\
& \times \prod_{\left(i_{0} \cdots i_{d-1}\right) \in \partial M^{d}}\left(\mathcal{V}_{d-1}^{H, K}\right)^{s_{i_{0} 0 \cdots i_{d-1}}}\left(h_{i_{0}}, \ldots, h_{i_{d-1}} ; v_{i_{0} i_{1}}, v_{i_{1} i_{2}}, \ldots\right),
\end{aligned}
$$

where $\left[h_{i}, v_{i j}\right]$ represents the gauge equivalence classes. (Equivalently, we can sum over all configurations and divide by the number of equivalent configurations in each gauge equivalence class.)

We emphasize that, since the boundary theory is nonlocal with respect to the boundary sites, it is no longer meaningful to distinguish on-site from non-on-site symmetry, or anomaly-free from anomalous symmetry.

However, this system does have a global $G$-symmetry. To see this, let us consider a transformation generated by $h \in H$, given by

$$
\left(h_{i}, v_{i j}\right) \rightarrow\left(h h_{i}, h v_{i j} h^{-1}\right)
$$

if $i$ is on the boundary, and

$$
g_{i} \rightarrow r(h) g_{i}
$$

if $i$ is in the bulk. Clearly, such a transformation is actually a $G$ transformation in the bulk. On the boundary, since $\left(h_{i}, v_{i j}\right)$ and $\left(v h_{i}, v v_{i j} v^{-1}\right)$ are gauge equivalent for $v \in K, h$ and $h v$ generate the same transformation. So, the transformation on the boundary is given by the equivalence class $[h]$ under the equivalence relation $h \sim h v, v \in K$. Since $K$ is a normal subgroup of $H$, the equivalence classes form a group $H / K=G$. Thus, the transformation is also a $G$ transformation on the boundary. Such a transformation is a symmetry of the model, since

$$
\begin{aligned}
& \mathcal{V}_{d-1}^{H, K}\left(h h_{i_{0}}, \ldots, h h_{i_{d-1}} ; h v_{i_{0} i_{1}} h^{-1}, h v_{i_{1} i_{2}} h^{-1}, \ldots\right) \\
& \quad=\mathcal{V}_{d-1}^{H, K}\left(h_{i_{0}}, \ldots, h_{i_{d-1}} ; v_{i_{0} i_{1}}, v_{i_{1} i_{2}}, \ldots\right)
\end{aligned}
$$

where we have used the definition in Eq. (4.47). We note that $h v_{i j} h^{-1} \in K$, since $K$ is a normal subgroup of $H$. So, the partition function in Eq. (4.53) gives us a boundary effective theory that still has the $G$ global symmetry.

Now we can ask whether the ground state at the boundary breaks the $G$-symmetry or not. More generally, what is the dynamical property of such a boundary? Is it gapped? To answer such a question, we note that, on a triangulated $M^{d}$, in general,

$$
\prod_{\left(i_{0} \cdots i_{d}\right) \in M^{d}} \nu_{d}^{s_{i_{0} \cdots i_{d}}}\left(g_{i_{0}}, \ldots, g_{i_{d}}\right) \neq 1
$$

since $M^{d}$ has a boundary. But, we can show that if the boundary is simply connected, then 


$$
\mathrm{e}^{-\int_{M^{d}} \mathcal{L} d^{d} x}=\prod_{\left(i_{0} \cdots i_{d}\right) \in M^{d}} \nu_{d}^{s_{i_{0} \cdots i_{d}}}\left(g_{i_{0}}, \ldots, g_{i_{d}}\right) \prod_{\left(i_{0} \cdots i_{d-1}\right) \in \partial M^{d}}\left(\mathcal{V}_{d-1}^{H, K}\right)^{-s_{i_{0} \cdots i_{d-1}}}\left(h_{i_{0}}, \ldots, h_{i_{d-1}} ; v_{i_{0} i_{1}}, v_{i_{1} i_{2}}, \ldots\right)=1
$$

To show this, we first recall that only flat connections on the boundary contribute to the path integral. If the boundary is simply connected, this means that we can assume that $v_{i j}$ is pure gauge. So, by the gauge transformation in Eq. (4.50), we can set all $v_{i j}$ to 1 on the boundary:

$$
\begin{aligned}
& \prod_{\left(i_{0} \cdots i_{d}\right)} \nu_{d}^{s_{i_{0}} \cdots i_{d}}\left(g_{i_{0}}, \ldots, g_{i_{d}}\right) \prod_{\left(i_{0} \cdots i_{d-1}\right)}\left(\mathcal{V}_{d-1}^{H, K}\right)^{-s_{i_{0} \cdots i_{d-1}}}\left(h_{i_{0}}, \ldots, h_{i_{d-1}} ; v_{i_{0} i_{1}}, v_{i_{1} i_{2}}, \ldots\right) \\
& =\prod_{\left(i_{0} \cdots i_{d}\right)} \nu_{d}^{s_{i_{0}} \cdots i_{d}}\left(g_{i_{0}}, \ldots, g_{i_{d}}\right) \prod_{\left(i_{0} \cdots i_{d-1}\right)}\left(\mathcal{V}_{d-1}^{H, K}\right)^{-s_{i_{0} \cdots i_{d-1}}}\left(\tilde{h}_{i_{0}}, \ldots, \tilde{h}_{i_{d-1}} ; 1,1, \ldots\right) \\
& =\prod_{\left(i_{0} \cdots i_{d}\right)} \nu_{d}^{s_{i_{0} \cdots i_{d}}}\left(g_{i_{0}}, \ldots, g_{i_{d}}\right) \prod_{\left(i_{0} \cdots i_{d-1}\right)}\left(\mu_{d-1}^{H}\right)^{-s_{i_{0}} \cdots i_{d-1}}\left(\tilde{h}_{i_{0}}, \ldots, \tilde{h}_{i_{d-1}}\right),
\end{aligned}
$$

where $\tilde{h}_{i}$ is obtained from $h_{i}$ by the gauge transformation that sets the $v_{i j}$ to 1 . But, this is 1 by virtue of Eq. (4.37).

The fact that the action amplitude of our theory on $M^{d}$ is always 1 if the boundary of $M^{d}$ is simply connected is enough to show that the system on $M^{d}$ is in a gapped phase both in the bulk and on the boundary. Such a gap state is the $K$-gauge deconfined state, described by the flat $K$-connection $v_{i j} \in K$ on each link. Also, $h_{i}$ and $g_{i}$ are strongly fluctuating and are quantum disordered as well. This is because the action amplitude is always equal to 1 regardless of the values of $h_{i}$ and $g_{i}$ (say, in the $v_{i j}=1$ gauge discussed above). So the partition function in Eq. (4.53) gives us a boundary of the SPT state that is in the deconfined phase of $K$-gauge theory and does not break the $G$-symmetry.

\section{G. The fourth boundary of a generic SPT state: A gapped symmetric boundary that preserves locality with emergent (soft) gauge fields}

In the last section, we constructed a gapped symmetric boundary of a SPT state by making its boundary nonlocal. In this section, we are going to fix this problem by constructing the fourth gapped symmetric boundary of a SPT state without changing the symmetry and without destroying the locality. The new gapped symmetric boundary has emergent gauge fields and topological order on the boundary. By this explicit construction, we show that in $3+1 \mathrm{D}$ and any higher dimensions, a SPT state with a finite group symmetry, regardless of unitary or antiunitary symmetry, always [63] has a gapped local boundary with the same symmetry.

The construction in this section is a generalization of the construction in Sec. III D.

To construct a local boundary, we replace $v_{i j}$ on a link by two degrees of freedom $h_{i j} \in H$ and $h_{j i} \in H$. In other words, a link $(i j)$ on the boundary $\partial D^{d}$ now carries two degrees of freedom $h_{i j} \in H$ and $h_{j i} \in H$ (see Fig. 14). We regard $h_{i}, h_{i j}, h_{i l}, \ldots$ as the degrees of freedom on site $i$ of the boundary (see Fig. 14). In the bulk, a site $i$ only carries a degree of freedom described by $g_{i}$.

We choose the action amplitude for our fourth boundary to be

$$
\begin{aligned}
\mathrm{e}^{-\int_{D^{d}} \mathcal{L} d^{d} x}= & \prod_{\left(i_{0} \cdots i_{d}\right) \in D^{d}} \nu_{d}^{s_{i_{0} \cdots i_{d}}}\left(g_{i_{0}}, \ldots, g_{i_{d}}\right) \\
& \times \prod_{\left(i_{0} \cdots i_{d-1}\right) \in \partial D^{d}}\left(\mathcal{V}_{d-1}^{H, K}\right)^{-s_{i_{0} \cdots \cdot i_{d-1}}} \\
& \times\left(h_{i_{0}}, \ldots, h_{i_{d-1}} ; h_{i_{0} i_{1}}, h_{i_{1} i_{0}}, \ldots\right) .
\end{aligned}
$$

In the following, we will define $\mathcal{V}_{d-1}^{H, K}$. We introduce a new form of cochain $\mathcal{V}_{d-1}^{H, K}$ encoding "soft-gauge fields" emergent from the local boundary sites that we prescribe below.

\section{A new cochain that encodes "emergent soft-gauge fields"}

First, we assume that $\mathcal{V}_{d-1}^{H, K}\left(h_{i_{0}}, \ldots, h_{i_{d-1}} ; h_{i_{0} i_{1}}, h_{i_{1} i_{0}}, \ldots\right)=$ 0 for any configurations $h_{i j}$ that do not satisfy

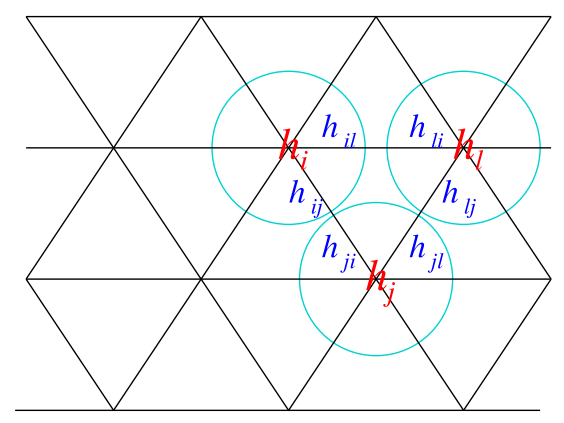

FIG. 14. A boundary of a $G$-SPT state. A vertex $i$ on the boundary carries $h_{i} \in H$, and a link $(i j)$ carries $h_{i j}$ and $h_{j i}$. The degrees of freedom in a circle, $h_{i}, h_{i j}, h_{i l}, \cdots$, belong to the same site labeled by $i$. 


$$
v_{i j} \equiv h_{i j} h_{j i}^{-1} \in K
$$

for every link or do not satisfy

$$
v_{i_{1} i_{2}} v_{i_{2} i_{3}}=v_{i_{1} i_{3}}
$$

for every triangle. So, only the $h_{i j}$ configurations that satisfy

$$
v_{i_{1} i_{2}} v_{i_{2} i_{3}}=v_{i_{1} i_{3}}, \quad v_{i j}=h_{i j} h_{j i}^{-1} \in K,
$$

on every triangle contribute to the path integral. Here, $v_{i j}$ corresponds to the $K$-gauge connection introduced in the last section.

The $K$-gauge symmetry will impose the equivalence relation

$$
\left(h_{i}, h_{i j}\right) \sim\left(k_{i} h_{i}, k_{i} h_{i j}\right),
$$

for any $k_{i} \in K$. The total number of inequivalent configurations on space-time boundary $\partial M^{d}$ is given by

$$
\frac{|H|^{N_{v}+2 N_{l}}}{|K|^{N_{v}}} .
$$

The exponent in the number of configurations is linear in $N_{v}$ and $N_{l}$, implying that the system is local.

Let us further assume that $\mathcal{V}_{d-1}^{H, K}\left(h_{0}, \ldots, h_{d-1}\right.$; $\left.h_{01}, h_{10}, \ldots\right)$ depends on $h_{i j}$ only via $v_{i j}=h_{i j} h_{j i}^{-1}$. So, we can express $\mathcal{V}_{d-1}^{H, K}\left(h_{0}, \ldots, h_{d-1} ; h_{01}, h_{10}, \ldots\right)$ as $\mathcal{V}_{d-1}^{H, K}\left(h_{0}, \ldots, h_{d-1} ; v_{01}, v_{02}, v_{12}, \ldots\right)$. We can simplify this further: The nonzero $\mathcal{V}_{d-1}^{H, K}\left(h_{0}, \ldots, h_{d-1} ; v_{01}, v_{02}, v_{12}, \ldots\right)$ can be expressed via $\mathcal{V}_{d-1}^{H, K}\left(h_{0}, \ldots, h_{d-1} ; v_{01}, v_{12}, \ldots\right.$, $\left.v_{d-2, d-1}\right)$. In other words, $v_{i j}$ on all the links of a $(d-1)$-simplex can be determined from a subset $v_{01}, v_{12}, \ldots, v_{d-2, d-1}$.

At this stage, we simply define $\mathcal{V}_{d-1}^{H, K}$ via Eq. (4.47), but using the effective gauge fields $v_{i j}$ defined in Eq. (4.63) to replace the hard-gauge fields that were assumed previously. The resulting model is manifestly gauge invariant, just as it was before. However, hard gauging has now been replaced with soft gauging, making the model completely local, both in the bulk and on the boundary. In this case, the global symmetry $G$ is on-site for the whole system (including bulk and boundary). But, if we integrate out the gapped bulk and consider only the effective boundary theory, we would like to ask if the effective global symmetry $G$ on the boundary is on-site or not. Since this point is important, we elaborate on it in the next section.

\section{The locality and effective non-on-site symmetry for the boundary theory}

We have shown that the model obtained by soft gauging is local both in the bulk and on the boundary. If we integrate out the bulk degrees of freedom, we get an effective boundary theory, whose action amplitude is given by a product of terms defined for each boundary simplex. The total boundary action amplitude is invariant under the $G$-symmetry transformation on the boundary, but each local term on a single boundary simplex may not be. This leads to a possibility that the effective boundary $G$-symmetry is not on-site. We have constructed two boundaries that are local in Secs. IV B and IV D. The first boundary in Sec. IV B has a non-on-site effective $G$ symmetry on the boundary, while the second boundary in Sec. IV D has an on-site effective $H$-symmetry on the boundary.

In the path integral, we only sum over gauge distinct configurations:

$$
\begin{aligned}
Z= & \sum_{\left\{g_{i},\left[h_{i}, h_{i j}\right]\right\}} \prod_{\left(i_{0} \cdots i_{d}\right) \in D^{d}} \nu_{d}^{s_{i_{0} \cdots i_{d}}}\left(g_{i_{0}}, \ldots, g_{i_{d}}\right) \\
& \times \prod_{\left(i_{0} \cdots i_{d-1}\right) \in \partial D^{d}}\left(\mathcal{V}_{d-1}^{H, K}\right)^{s_{i_{0} \cdots i_{d-1}}}\left(h_{i_{0}}, \ldots, h_{i_{d-1}} ; h_{i_{0} i_{1}}, h_{i_{1} i_{0}}, \ldots\right),
\end{aligned}
$$

where $\left[h_{i}, h_{i j}\right]$ represents the gauge equivalence classes.

Such a lattice gauge theory with soft gauging will have an on-site global symmetry $G$. To see this, let us consider a transformation generated by $h \in H$ on site $i$. It is given by, if $i$ is on the boundary,

$$
\left(h_{i}, h_{i j}\right) \rightarrow\left(h h_{i}, h h_{i j}\right)
$$

and, if $i$ is in the bulk,

$$
g_{i} \rightarrow r(h) g_{i}
$$

Such a transformation is a $G$ transformation in the bulk. On the boundary, since $\left(h_{i}, h_{i j}\right)$ and $\left(v h_{i}, v h_{i j}\right)$ are gauge equivalent for $v \in K, h$ and $h v$ generate the same transformation. So, the transformation on the boundary is given by the equivalence class $[h]$ under the equivalence relation $h \sim h v, v \in K$. Since $K$ is the normal subgroup of $H$, the equivalence classes form a group $H / K=G$. Thus, the transformation is also a $G$ transformation on the boundary. Such a transformation is onsite and is a symmetry of the model, since each term in the action amplitude, such as $\nu_{d}^{s_{i_{0} \cdots i_{d}}}\left(g_{i_{0}}, \ldots, g_{i_{d}}\right)$ and $\left(\mathcal{V}_{d-1}^{H, K}\right)^{s_{i_{0} \cdots i_{d-1}}}\left(h_{i_{0}}, \ldots, h_{i_{d-1}} ; h_{i_{0} i_{1}}, h_{i_{1} i_{0}}, \ldots\right)$, is invariant under the $G$-symmetry transformation: $\nu_{d}^{s_{i} \cdots i_{d}}\left(g g_{i_{0}}, \ldots, g g_{i_{d}}\right)=$ $\nu_{d}^{s_{i_{0} \cdots i_{d}}}\left(g_{i_{0}}, \ldots, g_{i_{d}}\right)$ and 


$$
\begin{aligned}
\mathcal{V}_{d-1}^{H, K}\left(h h_{i_{0}}, \ldots, h h_{i_{d-1}} ; h h_{i_{0} i_{1}}, h h_{i_{1} i_{0}}, \ldots\right) \\
=\mathcal{V}_{d-1}^{H, K}\left(h h_{i_{0}}, \ldots, h h_{i_{d-1}} ; h v_{i_{0} i_{1}} h^{-1}, h v_{i_{1} i_{2}} h^{-1}, \ldots\right) \\
\quad[\text { used the definition in Eq. }(4.61)] \\
=\mathcal{V}_{d-1}^{H, K}\left(h_{i_{0}}, \ldots, h_{i_{d-1}} ; v_{i_{0} i_{1}}, v_{i_{1} i_{2}}, \ldots\right) \\
\quad[\text { used the definition in Eq. }(4.47)] \\
=\mathcal{V}_{d-1}^{H, K}\left(h_{i_{0}}, \ldots, h_{i_{d-1}} ; h_{i_{0} i_{1}}, h_{i_{1} i_{0}}, \ldots\right) .
\end{aligned}
$$

To see if the effective boundary $G$-symmetry is on-site or not, we first note that the term in the total action amplitude, $\prod_{\left(i_{0} \cdots i_{d-1}\right) \in \partial D^{d}}\left(\mathcal{V}_{d-1}^{H, K}\right)^{S_{i_{0} \cdots i_{d-1}}}\left(h_{i_{0}}, \ldots, h_{i_{d-1}} ; h_{i_{0} i_{1}}, h_{i_{1} i_{0}}, \ldots\right)$, is purely a boundary term. Each contribution from a single boundary simplex is already invariant under the $G$-symmetry transformation [see Eq. (4.69)]. So, such a term will not affect the on-site-ness of the effective boundary symmetry, and we can ignore it in our discussion.

The other term $\prod_{\left(i_{0} \cdots i_{d}\right) \in D^{d}} \nu_{d}^{s_{i_{0} \cdots i_{d}}}\left(g_{i_{0}}, \ldots, g_{i_{d}}\right)$ may lead to non-on-site effective boundary symmetry. But the calculation is identical to that in Sec. IV C. We find that the resulting effective boundary $G$-symmetry is indeed non-onsite if the $G$-cocycle $\nu_{d}\left(g_{i_{0}}, \ldots, g_{i_{d}}\right)$ is not a coboundary.

So, the partition function Eq. (4.66) gives us a boundary effective theory that still has the $G$-symmetry, as well as a local Hilbert space. (The boundary does not break or extend the $G$-symmetry.) But, the effective $G$-symmetry on the boundary is non-on-site (i.e., anomalous).

The dynamical properties of the soft gauging model in Sec. IV G are the same as in the hard gauging case in Sec. IV F, since the two path integrals are manifestly the same. In particular, this is a physically satisfactory construction of a symmetry-preserving gapped boundary of a bulk SPT phase with global symmetry $G$. The boundary is topologically ordered with emergent $K$-gauge symmetry. The $K$-gauge theory is in a deconfined phase, which we discuss further in Sec. IV H. The boundaries of the CZX model discussed in Sec. IIID and Appendix A 2 are examples of this general construction.

\section{H. Gapped boundary gauge theories: $G$-symmetry preserving $(2+1 \mathrm{D}$ boundary or above $)$ or $G$-spontaneous symmetry breaking (1+1D boundary)}

To identify the boundary $K$-gauge theory, we look more closely at the boundary factors in the path integral in Eq. (4.45). To understand the boundary theory in isolation, it is convenient to consider the case that all $g_{i}$ are equal to 1 , which ensures that the boundary spins are $K$ valued. The boundary theory is now just a theory of $K$-valued variables with an action amplitude that is given by the product over all boundary simplices of the generalized cochain $\mathcal{V}_{d-1}^{H, K}$ that was defined in Eq. (4.47).

If we choose the spacetime to be a $d$-ball $D^{d}$, then the action amplitude in Eq. (4.66) is always equal to 1 regardless the values of $\left\{g_{i}\right\}$ in the bulk and $\left\{h_{i}, h_{i j}\right\}$ 's on the boundary [that satisfy Eq. (4.63)]. Thus, the system on a spacetime $D^{d}$ is in a gapped phase both in the bulk and on the boundary. Such a gapped state is the $K$-gauge deconfined state, since the $K$-connections $v_{i j}=h_{i j} h_{j i}^{-1} \in K$ are always flat and $v_{i j} v_{j k} v_{k i}=1$.

Does such a $K$-gauge deconfined state spontaneously break the $G$-symmetry? We note that, except the combinations $v_{i j} v_{j k} v_{k i}$ that are not fluctuating, other combinations of $h_{i j}$ 's are strongly fluctuating and quantumly disordered. Also, $h_{i}$ and $g_{i}$ are strongly fluctuating and quantumly disordered. In fact, the model described by Eq. (4.66) has a local $G$-symmetry [64]: The action amplitude for configuration $\left(g_{i}, h_{i}, h_{i j}\right)$ is the same as the action amplitude for configuration $\left(g_{i}^{\prime}, h_{i}^{\prime}, h_{i j}^{\prime}\right)=$ $\left[r\left(\tilde{h}_{i}\right) g_{i}, \tilde{h}_{i} h_{i}, \tilde{h}_{i} h_{i j}\right]$, where $\tilde{h}_{i} \in H$ generate the local $G$-symmetry on gauge-invariant states. This is because the action amplitude is always equal to 1 regardless of the values of $h_{i}, g_{i}$, and $h_{i j}$ on a spacetime $D^{d}$ (as long as $v_{i j} v_{j k} v_{k i}=1$ is satisfied). This local $G$-symmetry allows us to show that any $G$-symmetry-breaking order parameter that can be expressed as a local function of $\left(g_{i}, h_{i}, h_{i j}\right)$ will have a short-range correlation.

However, such a result is not enough for us to show all $G$-symmetry-breaking order parameters that are local operators to have short-range correlations. This is because some local operators are not local functions of $\left(g_{i}, h_{i}, h_{i j}\right)$, such as the operator that corresponds to a breakdown of the flat-connection condition $v_{i j} v_{j k} v_{k i}=1$. On a $1+1 \mathrm{D}$ boundary, such kinds of local operators can change the holonomy of the $K$-gauge field around the space $S^{1}$ of the boundary. As discussed in Sec. IIID, it is the order parameter that changes the holonomy that acquires a long-range correlation.

Therefore, we need to find a more rigorous way to test the spontaneous breaking of the $G$-symmetry. One way to do so is to calculate the partition function in Eq. (4.53) on a spacetime $M^{d}$, which is given by the number of configurations that satisfy that the flat-connection condition $v_{i j} v_{j k} v_{k i}=1$ and the condition $v_{i j} \in K$. When $K$ is Abelian, we find the partition function to be [65]

$$
\begin{aligned}
Z\left(M^{d}\right)= & \frac{|G|^{N_{v}^{\text {Bulk }}}|H|^{N_{v}^{\text {Bdry }}}}{|K|^{N_{v}^{\text {Bdry }}}}|H|^{N_{l}^{\text {Bdry }}} \\
& \times \frac{|K|^{N_{v}^{\text {Bdry }}}}{|K|^{\left|\pi_{0}\left(\partial M^{d}\right)\right|}}\left|\operatorname{Hom}\left[\pi_{1}\left(\partial M^{d}\right), K\right]\right| .
\end{aligned}
$$

Let us explain the above result. The $g_{i}$ 's on the vertices in the bulk contribute the factor $|G|^{N_{v}^{\text {Bulk }}}$ to the total configurations, where $N_{v}^{\text {Bulk }}$ is the number of vertices in the bulk (not including the boundary). The $h_{i}$ 's on the vertices on the boundary contribute the factor $|H|^{N_{v}^{\text {Bdry }}}$ to the total configurations, where $N_{v}^{\text {Bdry }}$ is the number of vertices on the 
boundary. The $\left(h_{i j}, h_{j i}\right)$ of the link on the boundary can be labeled by $\left(h_{i j}, v_{i j}\right)$, where $h_{i j} \in H$ and $v_{i j} \in K$. The $h_{i j}$ 's contribute the factor $|H|^{N_{l}^{\mathrm{Bdry}}}$, where $N_{l}^{\mathrm{Bdry}}$ is the number of links on the boundary. The $v_{i j} \in K$ needs to satisfy the flatconnection condition $v_{i j} v_{j k} v_{k i}=1$, and the counting is complicated. When $K$ is Abelian, $v_{i j}$ 's contribute to a factor $\left[|K|^{N_{v}^{\text {Bdry }}} /|K|^{\left|\pi_{0}\left(\partial M^{d}\right)\right|}\right]$, which comes from $v_{i j}$ of the form $v_{i j}=v_{i} v_{j}^{-1}, v_{i}, v_{j} \in K$. But, those are only contributions from the "pure gauge" configurations. There is another factor $\left|\operatorname{Hom}\left[\pi_{1}\left(\partial M^{d}\right), K\right]\right|$, which is the number of inequivalent $K$-gauge flat connections on $\partial M^{d}$. Last, we need to divide out a factor $|K|^{N_{v}^{\text {Bdry }}}$ due to the $K$-gauge redundancy in Eq. (4.64).

The volume-independent partition function is given by

$$
Z^{\mathrm{top}}\left(M^{d}\right)=\frac{\left|\operatorname{Hom}\left[\pi_{1}\left(\partial M^{d}\right), K\right]\right|}{|K|^{\left|\pi_{0}\left(\partial M^{d}\right)\right|}},
$$

which is a topological invariant on spacetime with a vanishing Euler number [66]. If we choose $M^{d}=S^{1} \times$ $D^{d-1}$, then $Z^{\text {top }}\left(S^{1} \times D^{d-1}\right)$ will be equal to the ground-state degeneracy on $D^{d-1}$ space:

$$
\begin{aligned}
\operatorname{GSD}\left(D^{d-1}\right) & =Z^{\operatorname{top}}\left(S^{1} \times D^{d-1}\right) \\
& = \begin{cases}|K|, & \text { if } d=3(2+1 \mathrm{D}) \\
1 & \text { if } d>3 .\end{cases}
\end{aligned}
$$

Our strategy here is to test the ground-state degeneracy caused by spontaneous symmetry breaking, based on the degeneracy of a spatial sphere $S^{d-2}$ on the boundary of a spatial bulk $D^{d-1}$. Namely, we compute $\operatorname{GSD}\left(D^{d-1}\right)=$ $Z^{\text {top }}\left(S^{1} \times D^{d-1}\right)$. Our argument relies on the fact that no ground-state degeneracy on a spatial boundary sphere $S^{d-2}$ means no spontaneous symmetry breaking.

Here, we show that on a $1+1 \mathrm{D}$ spatial boundary $S^{1}$ of a $2+1 \mathrm{D}$ bulk, the GSD is $|K|$, and we cannot exclude the possibility of spontaneous $G$-symmetry breaking. On a $2+1$ D spatial boundary $S^{2}$ of a $3+1$ D bulk, or any higher dimensions, the GSD is 1 , and there is no spontaneous $G$-symmetry breaking.

We note that our result here on the spontaneous symmetry breaking of $1+1 \mathrm{D}$ deconfined $K$-gauge theory is consistent with other independent checks from the Hamiltonian approach of Sec. III C and Appendix A 2 d, and the field theory approach of Appendix D 22.

As explained in Sec. IV D 1, once all the variables are $K$ valued, $\mu_{d-1}^{H}$ reduces to a cocycle $\mu_{d-1}^{K}$ appropriate for a $K$-gauge theory. As a result, the boundary factor in the path integral in Eq. (4.53) or (4.66), when the $g_{i}$ are 1, is just the action amplitude of a $K$-gauge theory deformed with the cocycle $\mu_{d-1}^{K}$, as in Dijkgraaf-Witten theory. This is the boundary state that has been coupled to the bulk $G$-SPT phase to give a gapped symmetric boundary.

In general, not all variants of $K$-gauge theory can occur in this way, because there may be some $\mu_{d-1}^{K}$ that do not come from any $\mu_{d-1}^{H}$. Restriction from $H$ to $K$ gives a map $s: \mathcal{H}^{d-1}[H, U(1)] \rightarrow \mathcal{H}^{d-1}[K, U(1)]$. The versions of $K$-gauge theory that arise in our construction are the ones associated to classes that are in the image of $s$. In general, if a given version of $K$-gauge theory can arise by our construction as the gapped boundary of a given $G$-SPT state, it can arise in more than one way. The number of ways that this can happen is the kernel of $s$, which equals the number of classes in $\mathcal{H}^{d-1}[H, U(1)]$ that map to a given class in $\mathcal{H}^{d-1}[K, U(1)]$.

\section{FIND A GROUP EXTENSION OF $G$ THAT TRIVIALIZES A $G$-COCYCLE}

\section{A. Proof: Existence of a finite $K$-extension trivializing any finite $G$ 's $d$-cocycle in $H$ for $d \geq \mathbf{2}$}

The construction in the last section gives a symmetric gapped boundary for the $G$-SPT state associated to a $G$-cocycle $\nu_{d} \in \mathcal{H}^{d}[G, U(1)]$, provided that we can find an extension of $G$,

$$
1 \rightarrow K \rightarrow H \stackrel{r}{\rightarrow} G \rightarrow 1,
$$

such that the $G$-cocycle $\nu_{d}$ becomes trivial when pulled back to an $H$-cocycle by $r$. In this section, we will give an explicit construction of such an extension for any finite group $G$ and for any $G$-cocycle $\nu_{d}$ when $d \geq 2$. This approach works for $d$-cocycles with $d \geq 2$; thus, the bulk dimension of the $G$-SPT state has to be greater than or equal to $1+1 \mathrm{D}$. Based on this method, below we show that a suitable group extension always exists; thus, we prove that within group cohomology construction,

Statement 1: Any bosonic SPT state with a finite onsite symmetry group $G$, including both unitary and antiunitary symmetry, can have an $H$-symmetry-extended (or $G$-symmetry-preserving) gapped boundary via a nontrivial group extension by a finite $K$, given the bulk spacetime dimension $d \geq 2$.

To motivate the construction, we start with the non-onsite symmetry discussed in Sec. IV. We can make the nonon-site symmetry be on-site by splitting $g_{i}$ on each vertex on the boundary into several variables $g_{i}^{1}, g_{i}^{2}, \ldots$, etc., one for each attached simplex (see Fig. 15). In the Euclidean signature, we take the new evolution operator

$$
\left(\mathrm{e}^{-\hat{H}_{\text {Bdry }}}\right)_{\left\{\tilde{g}_{i}^{m}, \ldots\right\},\left\{g_{i}^{m}, \ldots\right\}}
$$

to be nonzero only when $g_{i}^{1}=g_{i}^{2}=g_{i}^{3}=\cdots$ on each vertex. In other words, if the condition $g_{i}^{1}=g_{i}^{2}=g_{i}^{3}=\cdots$ is not satisfied on some vertices, then the configuration will 


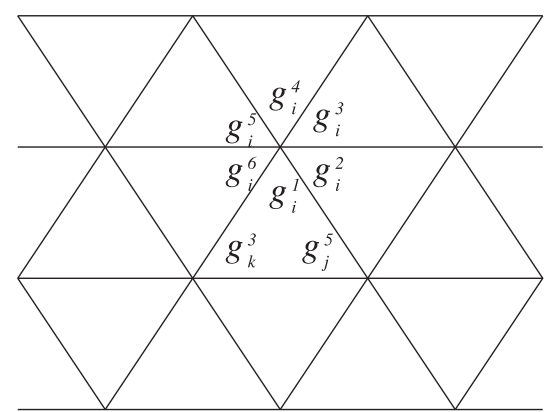

FIG. 15. On the boundary, we can split $g_{i}$ on each vertex into several $g_{i}^{1}, g_{i}^{2}, \cdots$, etc., one for each attached simplex.

correspond to high-energy boundary excitations on those vertices.

In the new boundary Hilbert space spanned by $\otimes_{i, m}\left|g_{i}^{m}\right\rangle$, the symmetry transformation

$\hat{U}(g)=\prod_{(i j \cdots k)} \hat{U}_{0}(g) \nu_{d}^{s_{i j \cdots k}}\left(g_{i}^{m_{i}}, g_{j}^{m_{j}}, \ldots, g_{k}^{m_{k}}, g^{*}, g^{-1} g^{*}\right)$

becomes on-site (or on-cell, or on-simplex). On each simplex, the symmetry transformation $\hat{U}(g)$ is given by

$$
\begin{aligned}
\hat{U}(g) & \left|g_{i}, g_{j}, \ldots, g_{k}\right\rangle \\
& =\hat{U}_{0}(g) \nu_{d}^{s_{i j \cdots k}}\left(g_{i}, g_{j}, \ldots, g_{k}, g^{*}, g^{-1} g^{*}\right)\left|g_{i}, g_{j}, \ldots, g_{k}\right\rangle \\
& =\nu_{d}^{s_{i j \cdots k}}\left(g_{i}, g_{j}, \ldots, g_{k}, g^{*}, g^{-1} g^{*}\right)\left|g g_{i}, g g_{j}, \ldots, g g_{k}\right\rangle .
\end{aligned}
$$

Thus, we can make any non-on-site symmetry on the boundary into an on-site symmetry, by redefining the boundary sites. This seems to contradict our picture that the non-on-site symmetry on the boundary captures the bulk SPT state, which should not be convertible into on-site boundary symmetry by any boundary operations (that have the local site structure).

In fact, there is no contradiction, since $\hat{U}(g), g \in G$ may not generate the group $G$. They may generate a bigger group $H$-an extension of $G$ by an Abelian group $K$. So, after we split $g_{i}$ into $g_{i}^{1}, g_{i}^{2}$, etc. on the boundary, the symmetry of our model is no longer $G$. It is changed into $H$. Since the symmetry transformation generated by $H$ is onsite, such a symmetry transformation is not anomalous. The bulk $G$-SPT state can also be viewed as an $H$-SPT state. But, as an $H$-SPT state, it is the trivial one, since the $H$-symmetry is on-site on the boundary.

So, we have found an extension of $G$, under $1 \rightarrow K \rightarrow H \stackrel{r}{\rightarrow} G \rightarrow 1$, where $K$ is an Abelian normal subgroup of $H$, such that

$$
\nu_{d}^{H}\left(h_{0}, \ldots, h_{d}\right) \in \mathcal{H}^{d}[H, U(1)],
$$

defined as

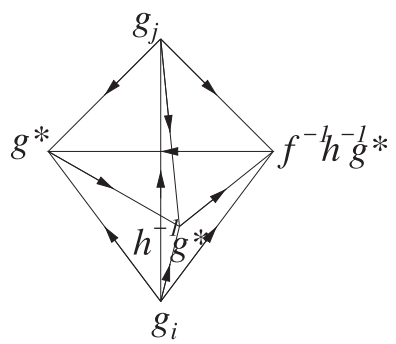

FIG. 16. Visualization for guiding the calculation in Eq. (5.7), shown here as three symmetry transformations [say, $h, f$, and $(f h)^{-1}$ ] on a $1+1 \mathrm{D}$ boundary of a $2+1 \mathrm{D}$ bulk.

$$
\nu_{d}^{H}\left(h_{0}, \ldots, h_{d}\right)=\nu_{d}\left[r\left(h_{0}\right), \ldots, r\left(h_{d}\right)\right],
$$

is trivial in $\mathcal{H}^{d}[H, U(1)]$. We also note that $K$ is a local symmetry (on each simplex) of the effective boundary Hamiltonian.

To calculate $K$ from $\nu_{d}\left(g_{i}, g_{j}, \ldots, g_{k}, g^{*}, g^{-1} g^{*}\right)$, we consider three symmetry transformations $h, f$, and $(f h)^{-1}$. We find that (see Fig. 16)

$$
\begin{aligned}
& \hat{U}\left[(f h)^{-1}\right] \hat{U}(f) \hat{U}(h) \\
&= \nu_{d}\left(f h g_{i}, f h g_{j}, \ldots, f h g_{k}, g^{*}, f h g^{*}\right) \\
& \times \nu_{d}\left(h g_{i}, h g_{j}, \ldots, h g_{k}, g^{*}, f^{-1} g^{*}\right) \\
& \times \nu_{d}\left(g_{i}, g_{j}, \ldots, g_{k}, g^{*}, h^{-1} g^{*}\right) \\
&= \nu_{d}\left(g_{i}, g_{j}, \ldots, g_{k}, h^{-1} f^{-1} g^{*}, g^{*}\right) \\
& \times \nu_{d}\left(g_{i}, g_{j}, \ldots, g_{k}, h^{-1} g^{*}, h^{-1} f^{-1} g^{*}\right) \\
& \times \nu_{d}\left(g_{i}, g_{j}, \ldots, g_{k}, g^{*}, h^{-1} g^{*}\right) \\
& \equiv \Phi_{h, f}\left(g_{i}, g_{j}, \ldots, g_{k}\right) .
\end{aligned}
$$

The above phase factor $\Phi_{h, f}\left(g_{i}, g_{j}, \ldots, g_{k}\right)$, as a function of $g_{i}, g_{j}, \ldots, g_{k}$, is a generator of the group $K$. We can obtain all the generators by choosing different $h$ and $f$ and, in turn, obtain the full group $K$. We note that the above construction is true only for $d \geq 2$.

Thus, this concludes our proof of Statement 1. We can rephrase it to the equivalent proved statements:

Statement 2: Any $G$-cocycle $\nu_{d}^{G} \in \mathcal{H}^{d}[G, U(1)]$ of a finite group $G$ (a bosonic SPT state with a finite, on-site, unitary or antiunitary symmetry, symmetry group $G$ ) can be pulled back to a finite group $H$ via a certain group extension $1 \rightarrow K \rightarrow H \stackrel{r}{\rightarrow} G \rightarrow 1$ by a finite $K$, such that $r^{*} \nu_{d}^{G}=\nu_{d}^{H}=\delta \mu_{d-1}^{H} \in \mathcal{H}^{d}[H, U(1)]$. Namely, a $G$-cocycle becomes an $H$-coboundary, split to $H$-cochains $\mu_{d-1}^{H}$, given the dimension $d \geq 2$ (q.e.d.).

Statement 3: Any $G$-anomaly in $(d-1) \mathrm{D}$ given by $\nu_{d}^{G} \in \mathcal{H}^{d}[G, U(1)]$ of a finite group $G$ can be pulled back to a finite group $H$ via a certain group extension $1 \rightarrow K \rightarrow$ $H \stackrel{r}{\rightarrow} G \rightarrow 1$ by a finite $K$, such that $G$-anomaly becomes $H$-anomaly free, given the dimension $d \geq 2$ (q.e.d.). 
Unfortunately, we do not have a systematic understanding of what $K$ will be generated by this construction. In particular, $K$ may be different for cocycles $\nu_{d}$ that differ only by coboundaries. Another drawback of this method is that we cannot obtain the exact analytic function of the split $H$-cochain easily.

However, we provide a different method that helps to derive the analytic $H$-cochain, based on the LydonHochschild-Serre spectral sequence in Appendix D3. Readers can find more systematic examples in Appendix D. Finally, we remark that very recently Ref. [67] has proven statements related to ours in a more mathematical setup [68].

\section{B. $2+1 / 1+1 \mathrm{D}$ and $d+1 / d \mathrm{D}$ bosonic SPTs for an even $d$ : The $d \mathrm{D} Z_{2}^{K}$-gauge theory boundary of $d+1 \mathrm{D}$ bulk} invariant $(-1)^{\int\left(a_{1}\right)^{d+1}}$ via $0 \rightarrow Z_{2} \rightarrow Z_{4} \rightarrow Z_{2} \rightarrow 0$

We would like to apply the above method to some cocycles that describes SPT states. For example, we can consider a nontrivial cocycle in $\nu_{3} \in \mathcal{H}^{3}\left[Z_{2}, U(1)\right]$ :

$\nu_{3}(-,+,-,+)=\nu_{3}(+,-,+,-)=-1, \quad$ others $=1$,

where $Z_{2}=\{+,-\}$. Choosing $g^{*}=+, h=-$, and $f=-$, we find

$$
\Phi_{--}\left(g_{i}, g_{j}\right)=\nu_{3}\left(g_{i}, g_{j},-,+\right) \nu_{3}\left(g_{i}, g_{j},+,-\right) .
$$

In fact, $\Phi_{--}\left(g_{i}, g_{j}\right)=\Phi_{-+}\left(g_{i}, g_{j}\right)=\Phi_{+-}\left(g_{i}, g_{j}\right)$, and

$$
\Phi_{h, f}(-,+)=\Phi_{h, f}(+,-)=-1, \quad \text { others }=1 .
$$

So, $K=Z_{2}$ and $H=Z_{4}$. The short exact sequence $0 \rightarrow Z_{2} \rightarrow$ $Z_{4} \rightarrow Z_{2} \rightarrow 0$ trivializes the cocycle $\nu_{3} \in \mathcal{H}^{3}\left[Z_{2}, U(1)\right]$.

See Appendix D 4 for further illumination of this example. In general, we find that, in any odd spacetime dimension, there is a $Z_{2}$-SPT phase and that a gapped symmetric boundary for this phase can be obtained from the extension $0 \rightarrow Z_{2}^{K} \rightarrow Z_{4}^{H} \rightarrow Z_{2}^{G} \rightarrow 0$. See Appendix D 5 . The bulk SPT phase is associated to the invariant $\exp \left(\mathrm{i} \pi \int a_{1} \cup a_{1} \cup \ldots \cup a_{1}\right) \equiv \exp \left[\mathrm{i} \pi \int\left(a_{1}\right)^{d+1}\right]$, with a cup product form of $a_{1} \cup a_{1} \cup \ldots \cup a_{1}$, a nontrivial element in $\mathcal{H}^{d+1}\left[Z_{2}, U(1)\right]$ for an even $d$. The $a_{1}$ here is a $\mathbb{Z}_{2}$-valued one-cocycle in $\mathcal{H}^{1}\left(M^{d+1}, \mathbb{Z}_{2}\right)$ on the spacetime complex $M^{d+1}$.

\section{C. $3+1 / 2+1 \mathrm{D}$ and $d+1 / d \mathrm{D}$ bosonic topological} superconductor with $Z_{2}^{T}$ time-reversal symmetry for an odd $d$ : The $d \mathrm{D} Z_{2}^{K}$-gauge theory boundary

$$
\begin{gathered}
\text { of } d+1 \mathrm{D} \text { bulk invariant }(-1)^{\int\left(w_{1}\right)^{d+1}} \\
\text { via } 0 \rightarrow Z_{2} \rightarrow Z_{4}^{T} \rightarrow Z_{2}^{T} \rightarrow 0
\end{gathered}
$$

Next, we consider a nontrivial cocycle $\nu_{4} \in$ $\mathcal{H}^{4}\left[Z_{2}^{T}, U_{T}(1)\right]=Z_{2}$ [15]. The $\nu_{4}$ represents a nontrivial class of bosonic SPTs with an antiunitary $G=Z_{2}^{T}$ timereversal symmetry. This SPT is also named a bosonic topological superconductor or bosonic topological paramagnet with $G=Z_{2}^{T}$. Here, $Z_{2}$ and $Z_{2}^{T}$ are the same group mathematically. However, the generator in $Z_{2}^{T}$ provides a nontrivial action on the $G$-module $U(1)$, denoted as $U_{T}(1)$. The subscript $T$ in the module $U_{T}(1)$ indicates that the group $Z_{2}^{T}$ has a nontrivial action on the module.

More generally, when a group $G$ contains an antiunitary operation such as time-reversal $Z_{2}^{T}$, we define a nontrivial $G$-module $U(1)$ as $U_{T}(1)$. We stress that $U(1)$ and $U_{T}(1)$ are the same Abelian group. The group action is only nontrivial when $g \cdot \nu=\nu^{s(g)}$, for $g \in G, \nu \in U_{T}(1)$, such that $s(g)=-1$ if $g$ contains an antiunitary element, and $s(g)=1$ if $g$ contains no antiunitary element. The formalism developed in this paper up to this point is applicable to this case, for models that fit in the group cohomology framework.

The group cocycle of this SPT phase is given by

$\nu_{4}(-,+,-,+,-)=\nu_{4}(+,-,+,-,+)=-1, \quad$ others $=1$,

where $Z_{2}^{T}=\{+,-\}$. Choosing $g^{*}=+, h=-$, and $f=-$, we find

$\Phi_{--}\left(g_{i}, g_{j}, g_{k}\right)=\nu_{4}\left(g_{i}, g_{j}, g_{k},-,+\right) \nu_{4}\left(g_{i}, g_{j}, g_{k},+,-\right)$,

and $\Phi_{--}\left(g_{i}, g_{j}, g_{k}\right)=\Phi_{-+}\left(g_{i}, g_{j}, g_{k}\right)=\Phi_{+-}\left(g_{i}, g_{j}, g_{k}\right)$. In fact, we obtain

$\Phi_{h, f}(-,+,-)=\Phi_{h, f}(+,-,+)=-1, \quad$ others $=1$.

So, $K=Z_{2}$ and $H=Z_{4}^{T}$. The short exact sequence $0 \rightarrow Z_{2} \rightarrow$ $Z_{4}^{T} \rightarrow Z_{2}^{T} \rightarrow 0$ trivializes the cocycle $\nu_{4} \in \mathcal{H}^{4}\left[Z_{2}^{T}, U_{T}(1)\right]$. This means that $\nu_{4}$ becomes a coboundary in $\mathcal{H}^{4}\left[Z_{4}^{T}, U_{T}(1)\right]$ for a larger group $H=Z_{4}^{T}$. Thus, we find that the $3+1 \mathrm{D}$ bosonic SPTs with $Z_{2}^{T}$-symmetry (the bosonic topological superconductor of $G=Z_{2}^{T}$ ) has a $2+1 \mathrm{D}$ symmetry-preserving surface $Z_{2}$ topological order.

For the boundary $K$-gauge theory of a $G$-SPT state, the gauge charge excitations are labeled by $\operatorname{Rep}(H)=\operatorname{Rep}\left(Z_{4}^{T}\right)$, with $H / K=G=Z_{4}^{T} / Z_{2}=Z_{2}^{T}$, instead of $\operatorname{Rep}(K \times G)=$ $\operatorname{Rep}\left(Z_{2} \times Z_{2}^{T}\right) . H$ is a "twisted" product of $K$ and $G$, the so-called projective symmetry group (PSG) introduced in Ref. [69]. When a gauge charged excitation is described by $\operatorname{Rep}(H)$ instead of $\operatorname{Rep}(K \times G)$, it implies that the particle carries a fractional quantum number of global symmetry $G$. We say there is a fractionalization of the symmetry $G$.

We note that the $e_{T} m_{T}$ surface topological order first proposed in Ref. [39] on the surface of a $3+1 D$ 
$Z_{2}^{T}$-bosonic topological superconductor is also a $2+1 \mathrm{D}$ deconfined $Z_{2}$ gauge theory.

See Appendix D 6 for further illumination of this example. In general, we find that the $0 \rightarrow Z_{2}^{K} \rightarrow Z_{4}^{T} \rightarrow$ $Z_{2}^{T} \rightarrow 0$ construction can provide a boundary $d \mathrm{D} Z_{2}^{K}$ gauge theory on $d+1 \mathrm{D}$ bosonic $Z_{2}^{T}$-SPTs, when $d$ is odd; see Appendix D 7. The bulk SPT invariant is equivalent to the partition function $\exp \left(\mathrm{i} 2 \pi \int \frac{1}{2} w_{1}^{d+1}\right)$ for an odd $d$, a nontrivial element in $\mathcal{H}^{d+1}\left[Z_{2}^{T}, U_{T}(1)\right]=\mathbb{Z}_{2}$. The $w_{1}$ here is $\mathbb{Z}_{2}$-valued, the first Stiefel-Whitney (SW) class in $\mathcal{H}^{1}\left(M^{d+1}, \mathbb{Z}_{2}\right)$ on the spacetime complex $M^{d+1}$. Here, $w_{1}=w_{1}\left(T M^{d+1}\right)$ is the $w_{1}$ of a spacetime tangent bundle over $M^{d+1} \cdot w_{1} \neq 0$ holds on a nonorientable manifold.

More examples of symmetry-extended gapped boundaries are provided in Appendix D.

\section{BOUNDARIES OF SPT STATES WITH FINITE OR CONTINUOUS SYMMETRY GROUPS AND BEYOND-GROUP COHOMOLOGY}

In the above Sec. V, we described a method that constructs an exactly soluble boundary for any withingroup-cohomology SPT states with a finite symmetry group $G$, via a nontrivial group extension by a finite group $K$. Those boundaries preserve the $G$-symmetry and have topological orders if the boundary dimension is $2+1 \mathrm{D}$ and higher. Such a result can be generalized to SPT states with a continuous compact symmetry group $G$, provided that the group cocycle that describes the $G$-SPT state can be trivialized by a finite extension $1 \rightarrow K \rightarrow H \rightarrow G \rightarrow 1$, namely, with a finite group $K$. This is because, even for a continuous compact symmetry group $G$, the action amplitude in Eq. (4.53) is still always equal to 1 regardless of the values of $\left\{g_{i}\right\}$ in the bulk and $\left\{h_{i}, h_{i j}\right\}$ 's on the boundary. Thus, Eq. (4.70) is still valid if we treat $|H|$ and $|G|$ as the volumes of the continuous group $H$ and $G$. When $K$ is finite, the flat condition $v_{i j} v_{j k} v_{k i}=1$ makes the $K$-gauge theory in a gapped deconfined phase. Therefore, for both a finite group $G$ and a continuous compact group $G$, a $d+1 \mathrm{D} G$-SPT state within group cohomology can have a symmetry-preserving gapped boundary if the $G$-group cocycle can be trivialized by a finite extension of $G$ and when $d \geq 3$.

The SPT states within group cohomology have pure gauge $G$-anomalies on the boundary corresponding to the global symmetry group $G$. More general SPT states exist that have mixed gauge-gravitational anomalies on the boundary [17]. Those SPT states are referred to as beyondgroup-cohomology SPT states [39]. Those beyond-groupcohomology SPT states can be constructed using the group cohomology of $G \times S O(\infty)$. More precisely, using the action amplitude constructed from the group cocycle $\nu_{d+1} \in \mathcal{H}^{d+1}[G \times S O(\infty), U(1)]$, we can construct models that realize the beyond-group-cohomology SPT states (as well as within-group-cohomology SPT states) in $d+1 \mathrm{D}$ [17]. However, the correspondence between $G \times S O(\infty)$-cocycle $\nu_{d+1}$ and a $d+1 \mathrm{D} G$-SPT state is not one to one: Several different cocycles can correspond to the same SPT state.

We note that [17]

$$
\begin{aligned}
\mathcal{H}^{d+1} & {[G \times S O(\infty), U(1)] } \\
= & \mathcal{H}^{d+1}[S O(\infty), U(1)] \\
& \oplus \bigoplus_{k=1}^{d+1} \mathcal{H}^{k}\left(G, \mathcal{H}^{d+1-k}[S O(\infty), U(1)]\right) .
\end{aligned}
$$

The cocycles in the first term $\mathcal{H}^{d+1}[S O(\infty), U(1)]$ describe invertible topological orders that do not need the symmetry group $G$. The cocycles in the second term $\bigoplus_{k=1}^{d+1} \mathcal{H}^{k}\left(G, \mathcal{H}^{d+1-k}[S O(\infty), U(1)]\right)$ will describe $G$-SPT states in a many-to-one fashion.

When $G$ is finite, a cocycle in $\bigoplus_{k=1}^{d+1} \mathcal{H}^{k}\left(G, \mathcal{H}^{d+1-k} \times\right.$ $[S O(\infty), U(1)])$ can always be trivialized by an Abelian extension $K: 1 \rightarrow K \rightarrow H \rightarrow G \rightarrow 1$. This is because, when $\mathcal{H}^{d+1-k}[S O(\infty), U(1)]=\mathbb{Z}_{N}$, then the $\mathcal{H}^{k}\left(G, \mathbb{Z}_{N}\right)$ can be viewed as a part of $\mathcal{H}^{k}[G, U(1)]$, and we can use the approach in Sec. V to show that the cocycles in $\mathcal{H}^{k}\left(G, \mathbb{Z}_{N}\right)$ can always be trivialized by a finite extension of $G$. When $\mathcal{H}^{d+1-k}[S O(\infty), \quad U(1)]=\mathbb{Z}$, we note that $\mathcal{H}^{k}(G, \mathbb{Z}) \cong$ $\mathcal{H}^{k-1}[G, U(1)]$. Using the approach in Sec. V, we can show that the cocycles in $\mathcal{H}^{k-1}[G, U(1)]$ can always be trivialized, which, in turn, allows us to show that the cocycles in $\mathcal{H}^{k}(G, \mathbb{Z})$ can always be trivialized.

This allows us to conclude that the bosonic $d+1 \mathrm{D}$ beyond-group-cohomology $G$-SPT states described by $\bigoplus_{k=1}^{d+1} \mathcal{H}^{k}\left(G, \mathcal{H}^{d+1-k}[S O(\infty), U(1)]\right)$ always have a symmetry-preserving gapped boundary when $G$ is finite and when the bulk space dimension $d \geq 3$. Here, $G$ can contain antiunitary symmetries, including time-reversal symmetry.

\section{BOUNDARIES OF BOSONIC OR FERMIONIC SPT STATES: COBORDISM APPROACH}

In principle, the philosophy of our approach should also work for the cobordism group description of topological states. For example, based on Ref. [19], one can consider bosonic SPTs in a $(d+1)$-dimensional spacetime with a finite internal on-site symmetry group $G$ via a cobordism theory. Such a SPT state is proposed to be classified by

$$
\begin{aligned}
\Omega_{\mathrm{tors}}^{d+1, S O}[B G, U(1)] & \equiv \Omega^{d+1, S O}[B G, U(1)] / \operatorname{im}\left(e_{G}\right) \\
& =\operatorname{Hom}\left[\Omega_{d+1, \mathrm{tors}}^{S O}(B G), U(1)\right],
\end{aligned}
$$

which is called the Pontryagin-dual of the torsion subgroup of the oriented bordism group $\Omega_{d+1}^{S O}(B G)$. In the first equality of Eq. (7.1), the $\Omega^{d+1, S O}[B G, U(1)]$ is called the 
oriented cobordism group of $B G$ with a $U(1)$ coefficient; it is defined as $\Omega^{d+1, S O}[B G, U(1)] \equiv \operatorname{Hom}\left[\Omega_{d+1}^{S O}(B G), U(1)\right]$, the space (here, an Abelian group) of homomorphisms from $\Omega_{d+1}^{S O}(B G)$ to $U(1)$. The $e_{G}$ is a map defined as $e_{G}: \operatorname{Hom}\left[\Omega_{d+1}^{S O}(B G), \mathbb{R}\right] \rightarrow \operatorname{Hom}\left[\Omega_{d+1}^{S O}(B G), U(1)\right] . \quad$ The image of the $e_{G}$ map is composed by elements of $\Omega^{d+1, S O}[B G, U(1)]$ that vanish on the torsion subgroup of the bordism group, $\Omega_{d+1 \text {, tors }}^{S O}(B G)$. Effectively, this yields the second equality; the $\Omega_{\text {tors }}^{d+1, S O}[B G, U(1)]$ is equivalent to $\operatorname{Hom}\left[\Omega_{d+1, \text { tors }}^{S O}(B G), U(1)\right]$, namely, the space (here, again, an Abelian group) of homomorphisms from the torsion subgroup of the bordism group $\Omega_{d+1, \text { tors }}^{S O}(B G)$ to $U(1)$.

To determine the symmetry-extended gapped interface of a $G$-SPT state, we need to find a larger total group $H$ that forms a group extension $1 \rightarrow K \rightarrow H \stackrel{r}{\rightarrow} G \rightarrow 1$ by a finite group $K$. By pulling $G$ back to $H$, we require that the nontrivial element in $\Omega_{\text {tors }}^{d+1, S O}[B G, U(1)]$ specifying a $G$-SPT state become a trivial identity element in the cobordism group $\Omega_{\text {tors }}^{d+1, S O}[B H, U(1)] \equiv$ $\Omega^{d+1, S O}[B H, U(1)] / \operatorname{im}\left(e_{H}\right)=\operatorname{Hom}\left[\Omega_{d+1, \text { tors }}^{S O}(B H), U(1)\right]$, where $e_{H}: \operatorname{Hom}\left[\Omega_{d+1}^{S O}(B H), \mathbb{R}\right] \rightarrow \operatorname{Hom}\left[\Omega_{d+1}^{S O}(B H), U(1)\right]$. In short, the $G$-SPT state within cobordism group $\Omega_{\text {tors }}^{d+1, S O}[B G, U(1)]$ becomes a trivial $H$-SPT state (a trivial vacuum in $H$ ) within cobordism group $\Omega_{\text {tors }}^{d+1, S O}[B H, U(1)]$. The boundary of such a $G$-SPT state should allow $G$ symmetry-preserving gapped interfaces with a deconfined topologically ordered $K$-gauge theory (where $K$ is a finite discrete group), if the spacetime dimensions of bulk dimension $d+1 \geq 4$, above or equal to $3+1 \mathrm{D}$.

The above procedure is for bosonic SPT states including only fundamental bosons. For fermionic SPT states including fundamental fermions, in principle, we can replace the oriented $S O$ in cobordism groups $\Omega^{d+1, S O}[B G, U(1)]$ and $\Omega^{d+1, S O}[B H, U(1)]$, to the Spin version of cobordism groups for the fermionic SPT states [namely, $\left.\Omega^{d+1, \operatorname{Spin}}[B G, U(1)]\right]$ and $\Omega^{d+1, \operatorname{Spin}}[B H, U(1)]$ ], and to the $\operatorname{Pin}^{ \pm}$version of cobordism groups for the fermionic SPT states with time-reversal symmetries [namely, $\Omega^{d+1, \operatorname{Pin}^{ \pm}}[B G, U(1)]$ and $\left.\Omega^{d+1, \operatorname{Pin}^{ \pm}}[B H, U(1)]\right]$, where $T^{2}=(-1)^{F}$ for $\mathrm{Pin}^{+}$or $T^{2}=+1$ for $\mathrm{Pin}^{-}$, respectively [21]. The $F$ is the fermion-number parity. In this setup, our approach for symmetric gapped interfaces should be applicable to both bosonic and fermionic SPT states. The underlying idea again is related to the fact that a certain global anomaly associated to $G$ on the boundary of $G$-SPT states becomes anomaly-free in a larger group $H$.

It will be interesting to find more concrete examples and figure out the explicit analytic (exactly soluble or not) lattice Hamiltonian construction for such symmetrypreserving gapped boundaries within the cobordism setup in the future.

\section{GENERIC GAPPED BOUNDARIES OR INTERFACES: MIXED SYMMETRY BREAKING, SYMMETRY EXTENSION, AND DYNAMICALLY GAUGING}

In this section, we will give an overview of how the symmetry extension construction we have described is related to what may be more familiar gapped boundary states. We will also describe the generalizations of the ideas to interfaces between SPT states, and to the case that the bulk phase has intrinsic topological order. We will further develop their path integrals, lattice Hamiltonians, and wave functions suitable for many-body quantum systems in Sec. IX.

\section{A. Relation to symmetry breaking}

The most familiar type of gapped boundary state for a $G$-SPT phase is obtained by explicitly or spontaneously breaking the $G$-symmetry on the boundary to a subgroup $H$ of $G$. Here, $H$ must have the property that the cocycle defining the $G$-SPT phase becomes a coboundary when the variables are restricted from $G$ to $H$. For the notational distinction, we call this unbroken subgroup $H$ of $G$ as $H=G^{\prime}$.

From the point of view of this paper, the statement that $G^{\prime}$ is a subgroup of $G$ means that there is an injective homomorphism $\imath: G^{\prime} \rightarrow G$. A gapped boundary state can be constructed if the given cohomology class in $\nu_{d}^{G} \in$ $\mathcal{H}^{d}[G, U(1)]$ is trivial when pulled back to $G^{\prime}$. See Appendix F 1 for explicit examples.

\section{B. Symmetry extension and mixed symmetry breaking or extension}

Our construction on the symmetry extension in this paper is instead based on a surjective, rather than injective, homomorphism $r: H \rightarrow G$. Because $r$ is surjective, the symmetry is extended (from $G$ to $H$ ) along the boundary, rather than being broken. By gauging $K=H / G$, one can arrange so that the global symmetry of the full system is $G$. Many examples of symmetry-extended gapped boundaries are shown in Appendix D.

It is straightforward to combine the two cases. We can construct a gapped boundary state associated to any homomorphism $\varphi: H \rightarrow G$, such that the cohomology class in $\mathcal{H}^{d}[G, U(1)]$ becomes trivial when pulled back to $H$. The construction proceeds exactly as we have explained in earlier sections of this paper, without any substantial modification. In this boundary state, $G$ is spontaneously or explicitly broken to the subgroup $G^{\prime}=\varphi(H)$, and then $G^{\prime}$ is extended to $H$.

More explicitly, one could also imagine arranging the above procedure in a two-stage process. Assume that, in a layer within a distance $\ell$ from the boundary, $G$ is spontaneously broken down to $G^{\prime}$. Then, near the boundary, the global or gauge symmetry is only $G^{\prime}$, and the boundary 
condition is defined by the choice of a group $H$, with a surjective map $r$ to $r(H)=G^{\prime}$, such that the cocycle of $G^{\prime}$ becomes trivial by lifting to $H$ : via $1 \rightarrow K^{\prime} \rightarrow H \stackrel{r}{\rightarrow} G^{\prime} \rightarrow 1$. In other words, to construct a boundary condition in a mixed symmetry-breaking or extension case, what we need is that the cocycle of $G$ that defines the bulk topological state, when restricted to $G^{\prime}$ and then pulled back to $H$, becomes trivial.

In all of these cases, one has to actually pick a trivialization of the pullback of $\nu_{d}^{G}$ to $H$. The possible choices differed by a class in $\mathcal{H}^{d-1}[H, U(1)]$ correspond to an $H$ topological state on the boundary. This corresponds roughly to appending an $H$-topological state on the boundary.

\section{Gapped interfaces}

One can similarly consider the case of an interface (i.e., domain wall) between two SPT phases. In general, we may have one symmetry group $G_{\mathrm{I}}$ on one side of the interface, with a cohomology class $\nu_{\mathrm{I}}$, and a second symmetry group $G_{\text {II }}$ on the other side, with its own cohomology class $\nu_{\mathrm{II}}$. (The gapped boundary of a $G$-topological state can be regarded as a gapped interface between a $G$-topological state and a trivial vacuum.) We shall describe gapped interfaces between these two states.

Interfaces can be reduced to boundary states by a wellknown folding trick. Instead of saying that there is $G_{\text {I }}$ on one side and $G_{\text {II }}$ on the other side, one "folds" along the interface and considers a system with a combined symmetry group $G=G_{\mathrm{I}} \times G_{\mathrm{II}}$ and a cohomology class $\nu_{\mathrm{I}} \times \nu_{\mathrm{II}}^{-1}$. (Folding inverts one of the two cohomology classes.) Then, we can construct gapped interfaces associated as above to any homomorphism $\varphi: H \rightarrow G_{\mathrm{I}} \times G_{\mathrm{II}}$.

An interesting special case is that the same group $G$ is supposed to be unbroken on both sides and also along the interface. This means that $G_{\mathrm{I}}=G_{\mathrm{II}}=G$, and that the unbroken subgroup $\varphi(H)$ is a diagonal subgroup $G^{\prime}$ of $G_{\mathrm{I}} \times G_{\mathrm{II}}$. The cohomology class $\nu_{\mathrm{I}} \times \nu_{\mathrm{II}}^{-1}$ of $G_{\mathrm{I}} \times G_{\mathrm{II}}=$ $G \times G$ restricts to a class of $G^{\prime}$ that we can denote by the same name. $H$ can be any finite extension of $G^{\prime} \cong G$ that trivializes this class.

\section{Intrinsic topological order}

Though our emphasis in this paper has been on gapped boundary states for SPT phases, a similar construction applies to bulk phases with intrinsic topological order.

We can construct such a phase simply by gauging the $G$-symmetry of a given $G$-SPT state. Then, since $G$ is extended to $H$ along the boundary, for consistency, we have to gauge the full $\mathrm{H}$-symmetry along the boundary. All our formulas make sense in that context.

SET phases can be treated in a similar way. For this, we gauge a subgroup $G_{0}$ of $G$. The most significant case is that $G_{0}=N$ is a normal subgroup of $G$. Then, gauging $N$ gives a state with intrinsic topological order of an $N$-gauge theory, in which $Q=G / N$ is a quotient group of global symmetries. Along the boundary, we have to gauge the inverse image of $N$ in $H$. If the map $\varphi: H \rightarrow G$ is surjective, then the $Q$-symmetry remains as a symmetry of the boundary state and is extended along the boundary to the inverse image of $Q$ in $H$. For details, see again Sec. IX. It is again possible to consider more general cases in which the $Q$-symmetry may be partly broken along the boundary and partly extended.

There is no essential loss of generality in assuming here that $G_{0}$ is a normal subgroup $N$ of $G$, for the following reason. If $G_{0}$ is not normal, then gauging $G_{0}$ will explicitly break $G$ to a subgroup $G^{*}$, the normalizer of $G_{0}$ in $G$. Then, $G_{0}$ is normal in $G^{*}$. After replacing $G$ by $G^{*}$, everything proceeds as before.

We provide other details of path integral or Hamiltonian models in Sec. IX. Many examples of dynamically gauging gapped boundaries or interfaces are provided in Appendix F.

\section{GENERAL CONSTRUCTION OF EXACTLY SOLUBLE LATTICE PATH INTEGRAL AND HAMILTONIAN OF GAPPED BOUNDARIES OR INTERFACES FOR TOPOLOGICAL PHASES IN ANY DIMENSION}

We consider the spacetime-lattice path integral formulation in Sec. IX A and the spatial lattice Hamiltonian formulation in Sec. IX B for a systematic construction of gapped boundaries or interfaces for topological phases in any dimension.

\section{A. Path integral}

In the following subsections, we systematically construct the path integral $Z$ defined for various topological phases (including SPT, gauge theory, SET, gapped boundary or interfaces, etc.) and contrast their properties. We shall clarify the gauge equivalent configuration briefly mentioned in Eq. (4.53) and the precise mod-out factor to remove the symmetry or gauge redundancy. In Sec. IV G, we showed the construction of cocycle $\left(\mathcal{V}_{d-1}^{H, K}\right)^{s_{i_{0} \cdots i_{d-1}}}\left(h_{i_{0}}, \ldots, h_{i_{d-1}}\right.$; $\left.h_{i_{0} i_{1}}, h_{i_{1} i_{0}}, \ldots\right)$ that contains the emergent gauge fields. We call this type of gauge field "soft gauged," which means that the Hilbert space of the gauge theory is still a tensor product form defined on each local site. $\mathcal{H}_{\text {tot }}=\otimes_{i} \mathcal{H}_{i}$, because the $h_{i}, h_{i j}, h_{i l}$ are variables assigned to the site $i$ (see Fig. 14). Below, we discuss the hard-gauged theory, where the total Hilbert space $\mathcal{H}_{\text {tot }} \neq \bigotimes_{i} \mathcal{H}_{i}$ is not a tensor product form of Hilbert spaces $\mathcal{H}_{i}$ on each local site $i$, since we require additional link variables.

We should note that we can easily formulate a soft-gauge theory from a hard-gauge theory, based on Sec. IV G. One reason to consider the hard-gauge theory in the following Secs. IX A 2 and IX A 3 is for the simplicity of notation and calculation, and for its smaller Hilbert space. 

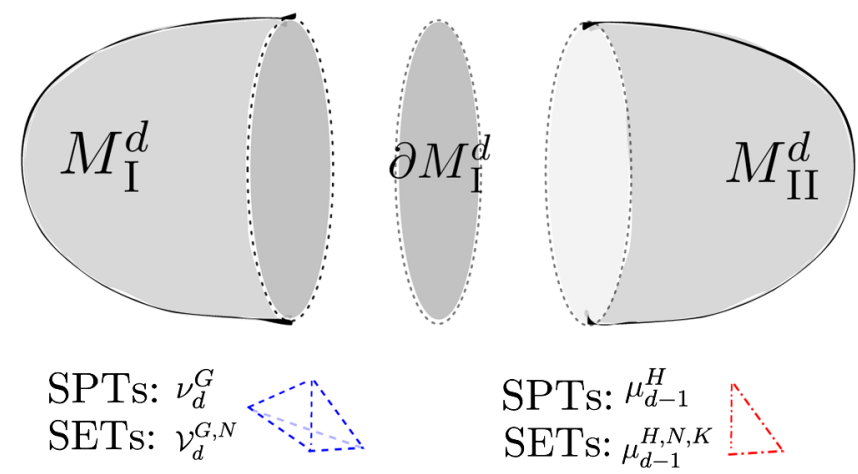

FIG. 17. In Sec. IX A, we define a lattice path integral on a $d$ dimensional spacetime manifold by triangulating the manifold to $d$-simplices. If the spacetime is closed, as in Secs. IX A 1, IX A 2, and IX A 3 , we assign $d$-simplices with cocycles $\nu_{d}^{G}$ for SPTs or with $\mathcal{V}_{d}^{G, N}$ for SETs. In this figure, the spacetime $M^{d}$ is obtained as the gluing of two manifolds $M_{\mathrm{I}}^{d} \cup M_{\mathrm{II}}^{d}$ with a common boundary $\partial M_{\mathrm{I}}^{d}$. For simplicity, we draw the $d=3$ case. One example of the $M^{3}=S^{3}$ is a three-sphere; then, we can choose $M_{\mathrm{I}}^{3}=D^{3}$ and $M_{\mathrm{II}}^{3}=D^{3}$, where the gapped spacetime boundary is on a twosphere $\partial M_{\mathrm{I}}^{3}=S^{2}$. We would like to define the path integral on an open manifold $M_{\mathrm{I}}^{d}$ with a gapped boundary $\partial M_{\mathrm{I}}^{d}$, details of which are discussed in Sec. IX A 4. In our construction, we assign lowerdimensional split cochains $\mu_{d-1}^{H}$ (or $\mathcal{V}_{d-1}^{H, K}$ ) for SPTs and $\mu_{d-1}^{H, N, K}$ for SETs to $(d-1)$-simplices paved onto a gapped boundary $\partial M_{\mathrm{I}}^{d}$.

Schematically, Figs. 17 and 18 summarize how to define an exactly soluble partition function or path integral on a triangulated spacetime complex. Normally, a path integral of a gapped topological phase is well defined on a closed spacetime manifold. However, here in particular, some path integral of a fully gapped topological phase is also well defined in the gapped bulk on $M_{\mathrm{I}}^{d}$ with a gapped interface $\partial M_{\mathrm{I}}^{d}$.

\section{SPTs on a closed manifold}

We start from reviewing and strengthening the understanding of a SPT path integral defined by homogeneous

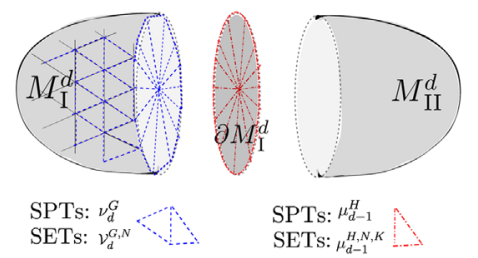

(a)

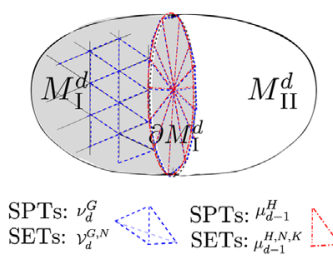

(b)
FIG. 18. Following Fig. 17, panel (a) shows the filling of $d$ cocycles into the gapped bulk in $M_{\mathrm{I}}^{d}$, and the filling of $(d-1)$ cochains onto a gapped boundary $\partial M_{\mathrm{I}}^{d}$. The combined result contributes to the topological amplitude shown in panel (b). Then, we need to sum over all the allowed group element configurations onto each vertex or link (the so-called "sum over all the colorings") to obtain the path integral $Z$. The explicit formula is derived in Sec. IX A 4.

$d$-cocycles $\nu_{d}\left(g_{i_{0}}, \ldots, g_{i_{d}}\right)$ of a cohomology group $\mathcal{H}^{d}[G, U(1)]$ for a global symmetry group $G[15]$ on a closed manifold,

$$
Z=\frac{1}{|G|^{N_{v, \text { Bulk }}}} \sum_{\left\{g_{i}\right\}} \prod_{\left(i_{0} \cdots i_{d}\right) \in M^{d}} \nu_{d}^{s_{i_{0} \cdots i_{d}}}\left(g_{i_{0}}, \ldots, g_{i_{d}}\right)
$$

We first assign the ordering of vertices as the branching structure; then, we assign a group element for each vertex as coloring. The sum over all possible colorings, by summing over all assignments of group elements, is done by $\sum_{\left\{g_{i}\right\}}$. On any closed manifold $M^{d}$, say, with a number of vertices $N_{v \text {,Bulk }}$, we can prove that the amplitude $\prod_{\left(i_{0} \cdots i_{d}\right) \in M^{d}} \nu_{d}^{s_{i_{0} \cdots i_{d}}}\left(g_{i_{0}}, \ldots, g_{i_{d}}\right)=1$ for any choice of $\left\{g_{i}\right\}$. Here is the proof: First, recall that the cocycle condition imposes that the cocycle $\prod \nu_{d}^{s_{i} \cdots i_{d}}\left(g_{i_{0}}, \ldots, g_{i_{d}}\right)=1$ on any closed sphere $S^{d}$. Second, we can simply connect every vertex $g_{j}$ on $M^{d}$ to an additional new point assigned with $g_{0}$ through a new edge $\overline{0 j}$, and we can view the amplitude as

$$
\begin{aligned}
\prod_{\left(i_{0} \cdots i_{d}\right) \in M^{d}} \nu_{d}^{s_{i \cdots i_{d}}}\left(g_{i_{0}}, \ldots, g_{i_{d}}\right) & =\prod_{\left(i_{0} \cdots i_{d}\right) \in M^{d}} \nu_{d}^{s_{i_{0} \cdots i_{d}}}\left(g_{i_{0}}, \ldots, g_{i_{d}}\right) \prod_{\left(j_{0} \cdots j_{d-1}\right) \in M^{d}} \nu_{d}^{s_{j_{0} \cdots j_{d-1}, 0}}\left(g_{j_{0}}, \ldots, g_{j_{d-1}}, g_{0}\right) \\
& =\prod_{\left(i_{0} \cdots i_{d}\right) \in M^{d}} \nu_{d}^{s_{i_{0} \cdots \cdots \cdots i_{d} 0}}\left(g_{i_{0}}, \ldots, \hat{g}_{i}, \ldots, g_{i_{d}}, g_{0}\right) \\
& =\prod_{\left(i_{0} \cdots i_{d}\right) \in M^{d}}\left(\delta \nu_{d}^{s_{i_{0} \cdots i \cdots i_{d} 0}}\left(g_{i_{0}}, \ldots, g_{i}, \ldots, g_{i_{d}}, g_{0}\right)\right)=\prod_{\left(i_{0} \cdots i_{d}\right) \in M^{d}} 1=1 .
\end{aligned}
$$

The first equality computes the amplitude from all vertices on $M^{d}$ and $g_{0}$, We use the fact that there are two terms under the same form $\nu_{d}\left(g_{j_{0}}, \ldots, g_{j_{d-1}}, g_{0}\right)$ overlapping the same $d$-simplex, with opposite orientations that cancel out. The second equality takes the product of each $d$-simplex, where $\hat{g}_{i}$ is a removed entry, where $i$ ranges from $\left\{i_{0}, \ldots, i_{d}, 0\right\}$. Moreover, the vertices $\left\{i_{0}, \ldots, i_{d}, 0\right\}$ and their connected edges also form a $d+1$-simplex. There are $d+1$ number of $d$-cocycles $\nu_{d}$ assigned to $d$-simplices paving on the surface of the $d+1$-simplex. Effectively, the surface $d+1$-simplex 
is a closed $S^{d+1}$ sphere, and the amplitude on $S^{d+1}$ yields a $d$-cocycle condition $\left[\delta \nu_{d}^{s_{i_{0} \cdots \cdots i_{d} 0}}\left(g_{i_{0}}, \ldots, g_{i}, \ldots, g_{i_{d}}, g_{0}\right)\right]=1$ in the third line. In Eq. (9.1), the product of amplitudes is 1 , and the summation $\sum_{\left\{g_{i}\right\}}$ yields a factor $\mid G^{N_{v, \text { Bulk }}}$ exactly canceling with the mod-out factor. We thus show that $Z=1$ on any closed manifold for SPT defined by homogeneous cocycles.

Global symmetry: We note that the global symmetry of SPT also manifests in the path integral. We first define the global symmetry transformation $\mathbf{g} \in G$ of SPT as sending each group element $g_{i} \rightarrow \mathbf{g} g_{i}$ on every vertex $i$. Through the homogeneous cocycle condition, $\mathbf{g} \cdot \nu_{d}\left(g_{i_{0}}, \ldots, g_{i_{d}}\right)=$ $\nu_{d}\left(\mathbf{g} \cdot g_{i_{0}}, \ldots, \mathbf{g} \cdot g_{i_{d}}\right)=\nu_{d}\left(g_{i_{0}}, \ldots, g_{i_{d}}\right) \quad[15]$; thus, $Z$ is invariant under the global symmetry transformation.

\section{Gauge theory with topological order on a closed manifold}

The gauge theory of a gauge group $K$ in this subsection is a topological gauge theory [22], suitable for certain topological orders. The path integral defined by inhomogeneous $d$-cocycles $\omega_{d}\left(k_{i_{0} i_{1}}, \ldots, k_{i_{d-1} i_{d}}\right) \in \mathcal{H}^{d}[K, U(1)]$ is

$Z=\frac{1}{|K|^{N_{v, \text { Bulk }}}} \sum_{\left\{k_{i_{j} i_{j+1}}\right\}} \prod_{\left(i_{0} \cdots i_{d}\right) \in M^{d}} \omega_{d}^{s_{i_{0} \cdots i_{d}}}\left(k_{i_{0} i_{1}}, \ldots, k_{i_{d-1} i_{d}}\right)$

on any closed manifold $M$. Each triangle (more generally, any contractible two-face or two-plaquette) must satisfy $k_{12} k_{23} k_{31}=1$ as a trivial element in $K$, which means a zero flux through a two-surface.

We note that the gauge theory $Z$ is not equal to 1 in general. The reason is that, on a manifold with noncontractible cycles such as $S^{1}$ circles, the inhomogeneous cocycles allow distinct gauge group elements winding through each cycle (that does not occur in homogeneous cocycles). This fact also reflects in nontrivial holonomies along noncontractible cycles for gauge field theory. However, we can show that $Z=1$ on $S^{d-1} \times S^{1}$. By considering the minimum triangulation that $S^{d-1}$ is the surface of a $d$-simplex, another $S^{1}$ connects each point back to itself. Each cocycle amplitude turns out to be 1, but the $\sum_{\{k\}}$ sums over group elements. The minimum triangulation of $S^{d-1} \times S^{1}$ has $N_{v}=d+1$ vertices and $N_{e}=d+1$ independent edge variables; thus, $Z=|K|^{N_{e}} /|K|^{N_{v}}=1$ on $S^{d-1} \times S^{1}$.

Gauge symmetry. - We note that the gauge symmetry also manifests in the path integral. We first define the local gauge-symmetry transformation $\mathbf{k} \in K$ on a particular site $j$, sending each group element on all the neighbor links through

$$
k_{i_{j} i_{j+1}} \rightarrow(\mathbf{k})^{-1} k_{i_{j} i_{j+1}}, \quad k_{i_{j-1} i_{j}} \rightarrow k_{i_{j-1} i_{j}}(\mathbf{k}) .
$$

Effectively what we do is equivalent to a Pachner move shifting the vertex $i_{j}$ to a new vertex $i_{j^{\prime}}$ with a new triangulation near this vertex, and we assign the link $\overline{i_{j} i_{j^{\prime}}}$ with a gauge transformation variable $\mathbf{k}=k_{i_{j} i_{j^{\prime}}} \in K$. We can focus on a local gauge transformation on a single site $i_{j}$; one can easily generalize to apply gauge transformations on every site. To prove that $Z$ is gauge invariant, we show that $\prod_{\left(i_{0} \cdots i_{d}\right) \in M^{d}} \omega_{d}^{s_{i_{0} \cdots i_{d}}}\left(k_{i_{0} i_{1}}, \ldots, k_{i_{d-1} i_{d}}\right)$ is gauge invariant. The ratio of amplitudes before and after gauge transformations is

$$
\begin{aligned}
& \frac{\prod_{\left(i_{0} \cdots i_{d}\right) \in M^{d}} \omega_{d}^{s_{i_{0} \cdots i_{d}}}\left(k_{i_{0} i_{1}}, \ldots, k_{i_{j-1} i_{j}}, k_{i_{j} i_{j+1}}, \ldots, k_{i_{d-1} i_{d}}\right)}{\prod_{\left(i_{0} \cdots i_{d}\right) \in M^{d}} \omega_{d}^{s_{i_{0} \cdots i_{d}}}\left[k_{i_{0} i_{1}}, \ldots, k_{i_{j-1} i_{j}}(\mathbf{k}),(\mathbf{k})^{-1} k_{i_{j} i_{j+1}}, \ldots, k_{i_{d-1} i_{d}}\right]} \\
& =\prod_{\left(\cdots i_{j} i_{j^{\prime}} \cdots\right) \in S^{d}} \omega_{d}^{s_{i, \ldots}}(\cdots)=(\delta \omega)_{d+1}=1 .
\end{aligned}
$$

In the first equality, we find that amplitudes around the vertex $i_{j}$ and $i_{j^{\prime}}$ are left over that cannot be directly canceled. There are two local patches centered around $i_{j}$ and $i_{j^{\prime}}$ as two $d$-dimensional disks $D^{d}$ and $D^{d}$. The two disks share the same boundary and can be glued to a sphere $S^{d}$. Thus, we can apply the $d$-cocycle condition $\delta \omega_{d}=1$ that the amplitude on $S^{d}$ is 1 , to prove that each amplitude in $Z$ is invariant. Local gauge transformation can be applied on every site, and the $Z$ is still gauge invariant by the same proof above.

\section{SETs on a closed manifold via $1 \rightarrow N \rightarrow G \rightarrow Q \rightarrow 1$, and a relation between SPTs and topologically ordered gauge theory}

Consider an anomaly-free SET path integral on a closed manifold under $1 \rightarrow N \rightarrow G \rightarrow Q \rightarrow 1$ [24,69]. Here, $G$ is a total symmetry group named a projective symmetry group (PSG), $N$ is a normal subgroup that can be dynamically gauged, and $Q$ is a quotient group of the remaining global symmetry [69]. We can regard the anomaly-free SET (well defined in its own dimensions) as gauging the $N$ normal subgroup in $G$-SPT in Sec. IX A 1:

$$
\begin{aligned}
Z= & \frac{1}{|G|^{N_{v, \text { Bulk }}}} \frac{1}{|N|^{N_{v, \text { Bulk }}}} \sum_{\left\{g_{i}, n_{i j}\right\}} \prod_{\left(i_{0} \cdots i_{d}\right) \in \partial M^{d}}\left(\mathcal{V}_{d}^{G, N}\right)^{S_{i_{0} \cdots i_{d}}} \\
& \times\left(g_{i_{0}}, \ldots, g_{i_{d}} ; n_{i_{0} i_{1}}, n_{i_{1} i_{2}}, \ldots, n_{i_{d-1} i_{d}}\right)
\end{aligned}
$$

with hard-gauge variables $n_{i_{j} i_{j+1}} \in N$ defined on the link or edge. The cocycle $\mathcal{V}^{G, N}$ can be rewritten in terms of homogeneous $G$ cocycle $\nu$ and inhomogeneous $G$ cocycle $\omega$ : 


$$
\begin{aligned}
\mathcal{V}_{d}^{G, N} & \left(g_{i_{0}}, \ldots, g_{i_{d}} ; n_{i_{0} i_{1}}, n_{i_{1} i_{2}}, \ldots, n_{i_{d-1} i_{d}}\right) \\
& =\nu_{d}^{G}\left(g_{i_{0}}, n_{i_{0} i_{1}} g_{i_{1}}, n_{i_{0} i_{1}} n_{i_{1} i_{2}} g_{i_{2}}, \ldots, n_{i_{0} i_{1}} \ldots n_{i_{d-1} i_{d}} g_{i_{d}}\right) \\
& =\omega_{d}^{G}\left(g_{i_{0}}^{-1} n_{i_{0} i_{1}} g_{i_{1}}, g_{i_{1}}^{-1} n_{i_{1} i_{2}} g_{i_{2}}, \ldots, g_{i_{d-1}}^{-1} n_{i_{d-1} i_{d}} g_{i_{d}}\right) .
\end{aligned}
$$

Gauge symmetry: - The cocycle $\mathcal{V}_{d}^{G, N}$ is invariant under the local gauge-symmetry transformation $\mathbf{n}_{j} \in N$ on each site for a gauge group $N$ :

$$
\begin{aligned}
g_{i_{j}} & \rightarrow\left(\mathbf{n}_{i_{j}}\right) \cdot g_{i_{j}}, \quad n_{i_{j} i_{j+1}} \rightarrow\left(\mathbf{n}_{i_{j}}\right) n_{i_{j} i_{j+1}}\left(\mathbf{n}_{i_{j+1}}\right)^{-1}, \\
n_{i_{j-1} i_{j}} & \rightarrow\left(\mathbf{n}_{i_{j-1}}\right) n_{i_{j-1} i_{j}}\left(\mathbf{n}_{i_{j}}\right)^{-1} .
\end{aligned}
$$

So, the $Z$ is invariant under the local gauge-symmetry transformation.

Global symmetry. - The cocycle $\mathcal{V}_{d}^{G, N}$ is invariant under a total symmetry transformation $\mathbf{g}$ of the symmetry group $G$ :

$$
g_{i_{j}} \rightarrow \mathbf{g} \cdot g_{i_{j}}, \quad n_{i_{j} i_{j+1}} \rightarrow(\mathbf{g}) n_{i_{j} i_{j+1}}(\mathbf{g})^{-1} .
$$

So, the $Z$ is invariant under the global symmetry transformation. The true global symmetry that does not include the gauge symmetry is the quotient group $G / N \equiv Q$.

The normalization in Eq. (9.5) has the $\left(|G|^{N_{v, \text { Bulk }}}\right)^{-1}$ modding out the site variables to make the path integral independent of the number of sites. The additional $\left(|N|^{N_{v, \text { Bulk }}}\right)^{-1}$ mods out the gauge transformation on each site through $\forall\left(\mathbf{n}_{j}\right) \in N$ to remove the gauge redundancy. It is easy to check that $Z\left[S^{d-1} \times S^{1}\right]$ as a path integral on $S^{d-1} \times S^{1}$ is always 1 , but, in general, $Z \neq 1$ for generic closed manifolds. If we choose that $N=1$ is trivial, then we reduce to a $G$-symmetric SPT in Sec. IX A 1 . If we choose that all $g_{j}=1$ are trivial, then we reduce to the gauge theory in Sec. IX A 2 of a gauge group $N$.

We can find a mapping between a $G$-symmetric SPT and a topologically ordered $G$-gauge theory, by the above $1 \rightarrow N \rightarrow G \rightarrow Q \rightarrow 1$ construction. For a $G$-symmetric SPT, we choose $N=1$ and $Q=G$. For a $G$-gauge theory, we choose $N=G$ and $Q=1$. This is a more general version of the relation between SPTs and topological order studied by Levin and $\mathrm{Gu}[23]$.

\section{Symmetry-extended boundary of a $G / N$-SET state via} $1 \rightarrow N \rightarrow G \rightarrow Q \rightarrow 1$ and $1 \rightarrow K \times N \rightarrow H \rightarrow Q \rightarrow 1$

Consider the $1 \rightarrow K \rightarrow H \stackrel{r}{\rightarrow} G \rightarrow 1$ formulation with $H / K=G$ in Appendix D 1 .

(1) Bulk G-SPTs on an open manifold with gapped boundary with extended $H$-symmetry action:

We consider a closed manifold $\mathcal{M}^{d}$ glued from two open manifolds: $M^{d}$ and its complement space $\mathcal{M}^{d} \backslash M^{d}$. Namely, $M^{d} \cup\left(\mathcal{M}^{d} \backslash M^{d}\right)=\mathcal{M}^{d}$, with a common $(d-1) \mathrm{D}$ boundary $\partial M^{d}$. We denote $N_{v \text {,Bulk }}$ as the number of vertices in $M^{d}$ but not on the boundary $\partial M^{d}$ nor on the complement $\left(\mathcal{M}^{d} \backslash M^{d}\right)$; each of these vertices has a dimension of Hilbert space $|G|$ on each site. We denote $N_{v \text {,Bdry }}$ as the number of vertices only on the boundary $\partial M^{d}$; each of these vertices has a dimension of Hilbert space $|H|$ on each site. We denote $N_{v \text {,Complt }}$ as the number of vertices on the complement $\left(\mathcal{M}^{d} \backslash M^{d}\right)$ but excluding the boundary $\partial M^{d}$; each of these vertices has again a dimension of Hilbert space $|H|$ on each site. The path integral is

$$
\begin{aligned}
& Z=\frac{1}{|G|^{N_{v, \text { Bulk }}}} \frac{1}{|H|^{N_{v, \text { Bdry }}+N_{v, \text { Complt }}}} \sum_{\left\{g_{i}, h_{i}\right\}} \prod_{\substack{\left(i_{0} \cdots i_{d}\right) \in M^{d} \\
\left(j_{0} \cdots j_{d}\right) \in \partial M^{d} \text { or } \mathcal{M}^{d} \backslash M^{d}}} \nu_{d}^{s_{i_{0} \cdots i_{d}}}\left(g_{i_{0}}, \ldots, g_{i_{d}}\right)\left(\nu_{d}^{H}\right)^{s_{j} \cdots \cdots j_{d}}\left(h_{j_{0}}, \ldots, h_{j_{d}}\right) \\
& =\frac{1}{|G|^{N_{v, \text { Bulk }}}} \frac{1}{|H|^{N_{v, \text { Bdry }}+N_{v, \text { Complt }}}} \sum_{\left\{g_{i}, h_{i}\right\}} \prod_{\substack{\left(i_{0} \cdots i_{d}\right) \in M^{d} \\
\left(j_{0} \cdots j_{d}\right) \in \partial M^{d} \text { or } \mathcal{M}^{d} \backslash M^{d}}} \nu_{d}^{s_{i_{0} \cdots i_{d}}}\left(g_{i_{0}}, \ldots, g_{i_{d}}\right)\left(\mu_{d-1}^{H}\right)^{s_{j 0} \cdots j_{d-1}}\left(h_{j_{0}}, \ldots, h_{j_{d-1}}\right) \\
& =\frac{1}{|G|^{N_{v, \text { Buk }}}} \frac{1}{|H|^{N_{v, \text { Bdry }}}} \sum_{\left\{g_{i}, h_{i}\right\}} \prod_{\substack{\left(i_{0} \cdots i_{d}\right) \in M^{d} \\
\left(j_{0} \cdots j_{d-1}\right) \in \partial M^{d}}} \nu_{d}^{s_{i_{0} \cdots i_{d}}}\left(g_{i_{0}}, \ldots, g_{i_{d}}\right)\left(\mu_{d-1}^{H}\right)^{s_{j_{0} \cdots j_{d-1}}}\left(h_{j_{0}}, \ldots, h_{j_{d-1}}\right) .
\end{aligned}
$$

Above, we applied Eq. (4.27), and the fact that the homogeneous cocycle $\nu_{d}^{H}\left(h_{0}, \ldots, h_{d}\right)=$ $\nu_{d}\left[r\left(h_{0}\right), \ldots, r\left(h_{d}\right)\right]$, which then split it to lowerdimensional homogeneous cochains $\mu_{d-1}^{H}$. Here, $\left(i_{0} \cdots i_{d}\right) \in M^{d}$ means the vertices in the bulk $M^{d}$ (with a total number $N_{v, \text { Bulk }}$ ), as well as on the boundary (with a total number $N_{v, \text { Bdry }}$ ). Here, $\left(j_{0} \cdots j_{d}\right) \in \partial M^{d}$ or $\mathcal{M}^{d} \backslash M^{d}$ means the vertices on the boundary $\partial M^{d}$ or in the complement $\mathcal{M}^{d} \backslash M^{d}$ with a total number $N_{v, \text { Bdry }}+N_{v \text {,Complt }}$. The cochains inside the volume of the complement $\mathcal{M}^{d} \backslash M^{d}$ cancel out to 1 due to overlapping terms with opposite orientations. An overall sum $\left(j_{0} \cdots j_{d}\right) \in \mathcal{M}^{d} \backslash M^{d}$ contributes a factor $|H|^{N_{v} \text {,Complt }}$ canceling with a normalizing factor to obtain Eq. (9.10). 
(2) Bulk G-SPT on an open manifold with gapped boundary anomalous SET (with a G-anomaly) of gauge group $K$ :

Consider a SPT path integral on an open manifold $M^{d}$ with gapped boundary anomalous SET on the $\partial M^{d}$. We can directly start from Eq. (9.9) and introduce gauge variables $k_{j j^{\prime}} \in K$ on the links between boundary sites on $\partial M^{d}$. After properly modding out the gauge redundancy, both obtain

$Z=\frac{1}{|G|^{N_{v, \text { Bulk }}}} \frac{1}{|H|^{N_{v, \text { Bdry }}}} \frac{1}{|K|^{N_{v, \text { Bdry }}}} \sum_{\left\{g_{i}, h_{i}, h_{i j}\right\}} \prod_{\left(i_{0} \cdots i_{d}\right) \in M^{d}} \nu_{d}^{s_{i 0} \cdots i_{d}}\left(g_{i_{0}}, \ldots, g_{i_{d}}\right) \prod_{\left(j_{0} \cdots j_{d-1}\right) \in \partial M^{d}}\left(\mathcal{V}_{d-1}^{H, K}\right)^{s_{j 0} \cdots j_{d-1}}\left(h_{j_{0}}, \ldots, h_{j_{d-1}} ; k_{j_{0} j_{1}}, k_{j_{1} j_{2}}, \ldots, k_{j_{d-2} j_{d-1}}\right)$.

The $\quad \mathcal{V}_{d-1}^{H, K}\left(h_{j_{0}}, \ldots, h_{j_{d-1}} ; k_{j_{0} j_{1}}, k_{j_{1} j_{2}}, \ldots, k_{j_{d-2} j_{d-1}}\right)=$ $\nu_{d-1}^{H}\left(h_{j_{0}}, k_{j_{0} j_{1}} h_{j_{1}}, \ldots, k_{j_{0} j_{1}} k_{j_{1} j_{2}} \ldots h_{j_{d-1}}\right)$ can be evaluated as homogeneous cochains by absorbing link variables to site variables.

(3) Bulk G-gauge theory on an open manifold with gapped boundary anomalous H-gauge theory:

We can gauge the global symmetry $G$ of Eq. (9.10) in the bulk to obtain the bulk $G$-gauge theory, while the boundary has an $\mathrm{H}$-gauge theory as an anomalous gapped boundary:

$$
\begin{aligned}
Z= & \frac{1}{|G|^{N_{v, \text {Buuk}}}} \frac{1}{|H|^{N_{v, \text { Bdry }}}} \\
& \times \sum_{\left\{g_{i j}, h_{i j}\right\}\left(i_{0} \cdots i_{d}\right) \in M^{d}} \omega_{d}^{s_{i_{0} \cdots i_{d}}}\left(g_{i_{0} i_{1}}, \ldots, g_{i_{d-1} i_{d}}\right) \\
& \times \prod_{\left(j_{0} \cdots j_{d-1}\right) \in \partial M^{d}}\left(\Omega_{d-1}^{H}\right)^{s_{j_{0} \cdots j_{d-1}}}\left(h_{j_{0} j_{1}}, h_{j_{1} j_{2}}, \ldots, h_{j_{d-2} j_{d-1}}\right) .
\end{aligned}
$$

The $\omega_{d}$ and $\Omega_{d-1}$ are an inhomogeneous cocycle and cochain suitable for gauge theories.

(4) Bulk SET on an open manifold with gapped boundary anomalous SET:

Alternatively, we can partially gauge a normal subgroup $N \subseteq G$ in the bulk $G$-SPTs and also on the boundary. Let us name the quotient group

$$
\frac{H}{K \times N}=\frac{G}{N} \equiv Q .
$$

This gives us a bulk SET with global symmetry $Q$ and gauge symmetry $N$ via

$$
1 \rightarrow N \rightarrow G \rightarrow Q \rightarrow 1 .
$$

The boundary anomalous SET with global symmetry $Q$ and gauge symmetry $K \times N$ is

$$
1 \rightarrow K \times N \rightarrow H \rightarrow Q \rightarrow 1 .
$$

Note that $1 \rightarrow K \rightarrow H \rightarrow G \rightarrow 1$.

\section{Symmetry-extended interface between two topological phases $G_{\mathrm{I}}$ and $G_{\mathrm{II}}$}

We construct a path integral of topological phases $G_{\mathrm{I}}$ and $G_{\text {II }}$ following Appendix D 2 a under $1 \rightarrow K \rightarrow H \stackrel{r}{\rightarrow} G_{\text {I }} \times$ $G_{\text {II }} \rightarrow 1$. First, consider a closed manifold $\mathcal{M}^{d}$ glued from two open manifolds: $M^{d}$ and its complement space $\mathcal{M}^{d} \backslash M^{d}$, with a common $(d-1) \mathrm{D}$ boundary $\partial M^{d}$. The $M^{d}$ is assigned with a Hilbert-space dimension $G_{\mathrm{I}} \times G_{\mathrm{II}}$ on each degree of freedom (on site or edge). The $\mathcal{M}^{d} \backslash M^{d}$ is originally assigned with $G_{\mathrm{I}} \times G_{\mathrm{II}}$-cocycles, but lifted to $H$ to become trivial coboundaries. Using the folding trick, given $\omega_{d}^{G_{\mathrm{I}} \times G_{\mathrm{II}}}(g)=\omega_{\mathrm{I}}^{G_{\mathrm{I}}}\left(g_{\mathrm{I}}\right) \cdot \omega_{\mathrm{II}}^{G_{\mathrm{II}}}\left(g_{\mathrm{II}}\right)^{-1}$, we can fold $\omega_{\text {II }}^{G_{\text {II }}}\left(g_{\text {II }}\right)$ to $-M^{d}$ with an opposite orientation, while we keep $\omega_{\mathrm{I}}^{G_{\mathrm{I}}}\left(g_{\mathrm{I}}\right)$ to $M^{d}$. The $M^{d} \cup\left(-M^{d}\right)$ can be glued to a closed manifold because they share the same boundary. We can define the path integral on a closed $M^{d} \cup\left(-M^{d}\right)$. More generally, we can call $M^{d}$ as $M_{\mathrm{I}}^{d}$, while we can modify the amplitude on $-M^{d}$ to a new amplitude on any open manifold $M_{\mathrm{II}}^{d}$, provided that $\partial M_{\mathrm{I}}^{d}=\partial M_{\mathrm{II}}^{d}=\partial M^{d}$ is the same common boundary. We denote the number of vertices $N_{v, \mathrm{I}}$ on $M_{\mathrm{I}}^{d}$, but not on $\partial M_{\mathrm{I}}^{d}$, and the similar definition for $N_{v, \text { II }}$ with I $\rightarrow$ II. We denote the number of vertices $N_{v, \partial}$ on $\partial M_{\mathrm{I}}^{d}=\partial M_{\mathrm{II}}^{d}$. We define this path integral on a closed spacetime $M_{\mathrm{I}}^{d} \cup M_{\mathrm{II}}^{d}$ below.

(1) Bulk $G_{I^{-}}$and $G_{I^{-}}$SPTs with gapped H-interface:

$$
\begin{aligned}
Z= & \frac{1}{\left|G_{\mathrm{I}}\right|^{N_{v, \mathrm{I}} \mid}|H|^{N_{v, \theta} \mid}\left|G_{\mathrm{II}}\right|^{N_{v, \mathrm{II}}}} \prod \sum_{\left\{g_{\mathrm{I}, i}\right\},\left\{h_{i}\right\},\left\{g_{\mathrm{II}, i}\right\}} \prod_{\left(i_{0} \cdots i_{d}\right) \in M_{\mathrm{I}}^{d}} \nu_{d}^{G_{\mathrm{I}} s_{i_{0} \cdots \cdots i_{d}}}\left(g_{\mathrm{I}, i_{0}}, \ldots, g_{\mathrm{I}, i_{d}}\right) \\
& \times \prod_{\left(i_{0} \cdots i_{d-1}\right) \in \partial M^{d}} \mu_{d-1}^{H_{i_{0} \cdots i_{d-1}}}\left(h_{i_{0}}, \ldots, h_{i_{d-1}}\right) \\
& \times \prod_{\left(i_{0} \cdots i_{d}\right) \in M_{\mathrm{II}}^{d}} \nu_{d}^{G_{\mathrm{II}} s_{i_{0}} \cdots i_{d}}\left(g_{\mathrm{II}, i_{0}}, \ldots, g_{\mathrm{II}, i_{d}}\right) .
\end{aligned}
$$


(ii) Bulk $G_{\mathrm{I}^{-}}$and $G_{\mathrm{II}^{-}}$SPTs with gapped boundary anomalous SET of gauge group $K$ :

$$
\begin{aligned}
Z= & \frac{1}{\left|G_{\mathrm{I}}\right|^{N_{v, \mathrm{I}} \mid}|H|^{N_{v, \partial} \mid}\left|G_{\mathrm{II}}\right|^{N_{v, \mathrm{II}}}} \\
& \times \sum_{\left\{g_{\mathrm{I}, i}\right\},\left\{h_{i}\right\},\left\{g_{\mathrm{II}, i}\right\}} \prod_{\left(i_{0} \cdots i_{d}\right) \in M_{\mathrm{I}}^{d}} \nu_{d}^{G_{\mathrm{I}} s_{i_{0}} \cdots i_{d}}\left(g_{\mathrm{I}, i_{0}}, \ldots, g_{\mathrm{I}, i_{d}}\right) \\
& \times \prod_{\left(j_{0} \cdots j_{d-1}\right) \in \partial M^{d}}\left(\mathcal{V}_{d-1}^{H, K}\right)^{s_{j_{0} \cdots j_{d-1}}} \\
& \times\left(h_{j_{0}}, \ldots, h_{j_{d-1}} ; k_{j_{0} j_{1}}, k_{j_{1} j_{2}}, \ldots, k_{j_{d-2} j_{d-1}}\right) \\
& \times \prod_{\left(i_{0} \cdots i_{d}\right) \in M_{\mathrm{II}}^{d}} \nu_{d}^{G_{\mathrm{II}} s_{i_{0}} \cdots i_{d}}\left(g_{\mathrm{II}, i_{0}}, \ldots, g_{\mathrm{II}, i_{d}}\right) .
\end{aligned}
$$

Here, we dynamically gauged the normal subgroup $K=H /\left(G_{\mathrm{I}} \times G_{\mathrm{II}}\right)$ on $\partial M^{d}$ by introducing the link variables along $\partial M^{d}$; thus, we rewrote $\mu_{d-1}^{H}$ into $\left(\mathcal{V}_{d-1}^{H, K}\right)$.

(3) Bulk SETs with gapped interface anomalous SET of enhanced gauge symmetry:

Developed from the above case 2 , bulk $G_{\mathrm{I}^{-}}$and $G_{\mathrm{II}}$-SPTs with gapped boundary anomalous SET of gauge group $K$, we can partially gauge normal subgroups of $G_{\mathrm{I}^{-}}$and $G_{\mathrm{II}^{-}}$SPTs, so that the bulk has SETs while the interface has an anomalous SET.

\section{B. Wave function and lattice Hamiltonian}

We would like to formulate a lattice Hamiltonian on the space lattice, whose time-dependent Schrödinger equation gives rise to the same low-energy physics governed by the path integral definition in the previous Sec. IX A. We motivate the Hamiltonian construction by thinking of ground-state wave functions. The lattice Hamiltonian below will be a SET generalization from the SPTs of Ref. [15] and the topological orders or gauge theories of Refs. [70,71]. Our Hamiltonian in Sec. IX B 2 is also a generalization of SETs of Ref. [72] to include a projective symmetry group under $G / N=Q$. We further implement anomalous SET gapped boundaries or interfaces in Sec. IX B 3.

Schematically, Figs. 19 and 20 summarize how to define an exactly soluble lattice Hamiltonian and wave function on a spatial manifold. Normally, a wave function of gapped topological phase is well defined on a closed spatial manifold. However, here in particular, some wave function of fully gapped topological phase can also be well defined in the gapped bulk on $R_{\mathrm{I}}$ with a gapped interface $\partial R$.

\section{Trivial product state and lattice Hamiltonian}

We can consider a total trivial product state wave function, where $\left\{g_{i}\right\}$ specifies the group element in a symmetry group $G$ and its assignment to a local site $i$ on a regularized $d \mathrm{D}$ spatial manifold $M$; the wave function has its coefficient: $\Phi_{0}\left(\left\{g_{i}\right\}_{M}\right)=1$. Its wave state vector in the Hilbert space is

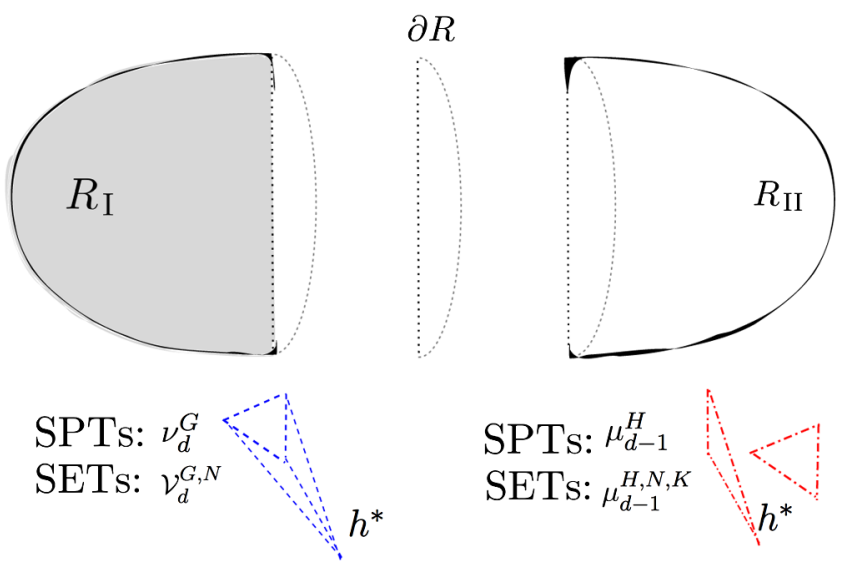

FIG. 19. In Sec. IX B, we define wave functions and lattice models on a $(d-1)$-dimensional space manifold by triangulating the manifold to $(d-1)$-simplices. If the space is closed, as in Sec. IX B 2, we assign $(d-1)$-simplices together with an extended vertex $h^{*}$, with cocycles $\nu_{d}^{G}$ for SPTs or with $\mathcal{V}_{d}^{G, N}$ for SETs. In this figure, the space is obtained as the gluing of two spatial manifolds $R_{\mathrm{I}} \cup R_{\mathrm{II}}$ with a common boundary $\partial R$. For simplicity, we draw the $d=3$ case. One example of the $R_{\mathrm{I}} \cup$ $R_{\mathrm{II}}=S^{2}$ is a two-sphere; then, we can choose $R_{\mathrm{I}}=D^{2}$ and $R_{\mathrm{II}}=D^{2}$, where the gapped spacetime boundary is on a onecircle $\partial R=S^{1}$. We would like to define the wave function on an open manifold $R_{\mathrm{I}}$ (shown in gray) with a gapped boundary $\partial R$ (shown as a dotted curve), where details are discussed in Sec. IX B 3. In our construction, we assign lower-dimensional split cochains $\mu_{d-1}^{H}\left(\right.$ or $\mathcal{V}_{d-1}^{H, K}$ ) for SPTs and $\mu_{d-1}^{H, N, K}$ for SETs to $(d-2)$-simplices connecting to the additional vertex $h^{*}$ paved onto a gapped boundary $\partial R$.

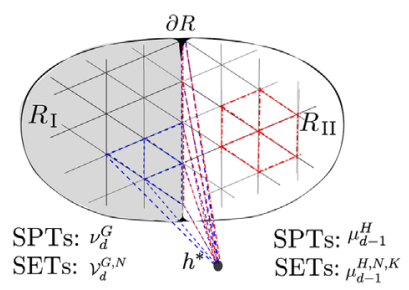

(a)

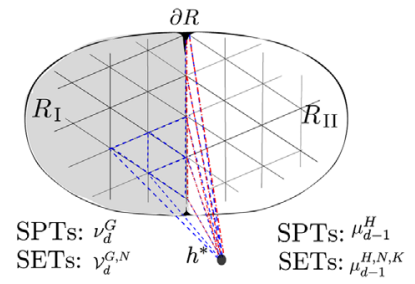

(b)
FIG. 20. Following Fig. 19, panel (a) shows that a wave function amplitude is the product of two contributions. The first contribution is the filling of $d$-cocycles into the gapped bulk in $R_{\mathrm{I}}$ connecting to $h^{*}$. The second contribution is the filling of $(d-1)$ cochains onto a gapped boundary $\partial R$ connecting to $h^{*}$ and into the surface of the other complement bulk $R_{\mathrm{II}}$. The combined result contributes to panel (b), where the $(d-1)$-cochains on the region $R_{\mathrm{II}}$ can be deformed to a trivial product state (as a trivial gapped vacuum) under local unitary transformations without breaking the global symmetry. We can remove the wave function amplitude on $R_{\mathrm{II}}$ after a proper amplitude normalization. Thus, the wave function is well defined simply in $R_{\mathrm{I}}$ and on $\partial R$. The explicit formula is derived in Sec. IX B 3. 


$$
\begin{aligned}
\left|\Phi_{0}\right\rangle & \propto \sum_{\left\{g_{i}\right\}_{M}} \Phi_{0}\left(\left\{g_{i}\right\}_{M}\right)\left|\left\{g_{i}\right\}_{M}\right\rangle=\sum_{\left\{g_{i}\right\}_{M}}\left|\left\{g_{i}\right\}_{M}\right\rangle \\
& =\left(\sum_{g_{1}}\left|g_{1}\right\rangle\right) \otimes\left(\sum_{g_{2}}\left|g_{2}\right\rangle\right) \cdots \otimes\left(\sum_{g_{i}}\left|g_{i}\right\rangle\right) \otimes \ldots,
\end{aligned}
$$

which we can properly normalize to have $\left\langle\Phi_{0} \mid \Phi_{0}\right\rangle=1$. Note that $\left|\left\{g_{i}\right\}_{M}\right\rangle$ has a tensor product structure, $\left|\left\{g_{i}\right\}_{M}\right\rangle=\ldots \otimes\left|g_{i}\right\rangle \otimes \ldots ;$ here, $i$ is the site index for some site $i$ distributed around the spatial manifold $M$. To see that the state vector is a trivial product state, we notice that it is indeed a tensor product of $\left(\sum_{g_{i}}\left|g_{i}\right\rangle\right)$ on each site $i$, where $\left(\sum_{g_{i}}\left|g_{i}\right\rangle\right)$ sums over all group element bases. The Hilbert space on each site $j$ is $\mathcal{H}_{j}$ with a Hilbert space dimension $|G|$ spanned by $\left|g_{j}\right\rangle$. The total Hilbert space is also a tensor product structure: $\mathcal{H}_{\text {total }}=\otimes_{j} \mathcal{H}_{j}$.

Considering the site index $j$, we can write down the exactly soluble Hamiltonian whose ground state is $\left|\Phi_{0}\right\rangle$ :

$$
\hat{H}_{j}=-\left|\phi_{j}\right\rangle\left\langle\phi_{j}\left|=-\sum_{g_{j} \in G}\right| g_{j}\right\rangle \sum_{g_{j}^{\prime} \in G}\left\langle g_{j}^{\prime}\left|=-\sum_{g_{j}, g_{j}^{\prime} \in G}\right| g_{j}\right\rangle\left\langle g_{j}^{\prime}\right| .
$$

Here, $\hat{H}_{j}=-\left|\phi_{j}\right\rangle\left\langle\phi_{j}\right|$ is a local operator on each site $j$, and $\left|\phi_{j}\right\rangle=\sum_{g_{j} \in G}\left|g_{j}\right\rangle$ is an equal-weight sum of all states of all group elements $g_{j}$ on each site. Thus, $\hat{H}_{j}=-\left|\phi_{j}\right\rangle\left\langle\phi_{j}\right|$ is proportional to a constant matrix $\left(\begin{array}{ccc}1 & 1 & \ldots \\ 1 & 1 & \ldots \\ \vdots & \vdots & \ddots\end{array}\right)$ in the group element basis $\left|g_{j}\right\rangle$ acting on each site. Thus, we construct a trivial product state and lattice Hamiltonian for a trivial insulator with a finite energy gap.

\section{Short-range or long-range entangled states and} SPT or topologically ordered or SET lattice Hamiltonians

Now, we consider gapped short-range or long-range entangled states for an anomaly-free Hamiltonian on a closed space that is well defined in a $d-1 \mathrm{D}$ spatial lattice. We can consider (1) a $G$-SPT for a cocycle $\nu_{d}^{G}$ in Sec. IX A 1, (2) an $\mathrm{N}$-gauge theory with intrinsic topological order for a cocycle $\omega_{d}^{N}$ in Sec. IX A 2, or (3) a SET prescribed by $1 \rightarrow N \rightarrow G \rightarrow Q \rightarrow 1$ for a cocycle $\mathcal{V}_{d}^{G, N}$ in Sec. IX A 3 .

The SET state in Sec. IX A 3 is the most general, containing all other cases by Eq. (9.6); thus, we focus on the SETs below. For a nontrivial, nonproduct state wave function of SETs, we define a particular wave function coefficient on a closed space $M$ as

$$
\begin{aligned}
& \Phi\left(\left\{g_{i}, n_{i j}\right\}_{M}\right) \\
& \quad \equiv \prod_{\{\ldots\}} \mathcal{V}_{d}^{G, N s_{i_{0} \ldots i_{*}}}\left(g_{i_{0}}, \ldots, g^{*} ; n_{i_{0} i_{1}}, n_{i_{1} i_{2}}, \ldots, n_{i_{d-1} i_{*}}\right),
\end{aligned}
$$

where $\left\{g_{i}, n_{i j}\right\}_{M}$ are a set of site $(i)$ and link $(i j)$ variables on $M$, for $g_{i} \in G$ and $n_{i j} \in N$. Conventionally, $\mathcal{V}_{d}^{G, N}$ is a $U(1)$ phase, except that we set $\mathcal{V}_{d}^{G, N}$ as zero if and only if any face of its simplex violates $n_{12} n_{23} n_{31}=1$. The $g^{*}$ is fixed and assigned to an additional fixed point $i_{*}$ outside $M$. There are link variables $n_{i_{j} i_{*}}$ from any site $j$ on $M$ to $i_{*}$. Given a wave function input parameter $\left\{g_{i}, n_{i j}\right\}_{M}$, to determine the wave function $\Phi\left(\left\{g_{i}, n_{i j}\right\}_{M}\right)$, the only input data we need are these two:

$$
g^{*}, \quad n_{i_{0} i_{*}} .
$$

We only need to provide another input data $n_{i_{0} i_{*}}$, as a link variable connecting a particular site $i_{0}$ to $i_{*}$. Any other variables $n_{i_{j} i_{*}}$ are determined by a zero flux condition through any closed loop $n_{i_{j} i_{*}} n_{i_{*} i_{0}} n_{i_{0} i_{j}}=1$, namely, $n_{i_{j} i_{*}}=n_{i_{j} i_{0}} n_{i_{0} i_{*}}$. Here, $\prod_{\{\ldots\}}$ is a product over all simplices assigned with cocycles. The zero flux condition through any closed loop constrains that the wave function has a trivial holonomy around any cycle of the closed manifold. Thus, we only generate a unique ground state so far. (We will comment how to generate other ground states with nontrivial holonomy for topological orders or SETs later.) This ground state as a vector in the Hilbert space is, up to a normalization,

$$
|\Phi\rangle \propto \sum_{\left\{g_{i}, n_{i j}\right\}_{M}} \Phi\left(\left\{g_{i}, n_{i j}\right\}_{M}\right)\left|\left\{g_{i}, n_{i j}\right\}_{M}\right\rangle .
$$

The $\left|\left\{g_{i}, n_{i j}\right\}_{M}\right\rangle$ has a tensor product structure, $\left|\left\{g_{i}, n_{i j}\right\}_{M}\right\rangle=\ldots \otimes\left|g_{i}\right\rangle \otimes \ldots \otimes\left|n_{i j}\right\rangle \otimes \ldots=\otimes_{i}\left|g_{i}\right\rangle \otimes_{i j}\left|n_{i j}\right\rangle$.

Now, we construct an exactly soluble Hamiltonian for the above gapped ground state as

$$
\hat{H}=-\sum_{v} \hat{A}_{v}-\sum_{f} \hat{B}_{f} .
$$

The first term, $\hat{A}_{v}$, acts on the wave function of a constanttime slice through each vertex $v$ in the space by lifting the initial state through an "imaginary time" evolution to a new state with a vertex $v^{\prime}$ via

$$
\hat{A}_{v}=\frac{1}{|G|} \frac{1}{|N|} \sum_{\substack{\left[v v^{\prime}=n \in N, g \in G\right.}} \hat{A}_{v}^{g, n}
$$

$$
\begin{aligned}
& \hat{A}_{v}^{g, n}\left|g_{v}, n_{i v}, n_{v j}, \ldots\right\rangle \\
& =\prod_{\{\ldots\}} \mathcal{V}_{d}^{G, N s_{\ldots}}\left(g, g_{v}, \ldots ; n, n_{i v} \cdot n, n^{-1} \cdot n_{v j}, \ldots\right) \\
& \quad \times\left|g, n_{i v} \cdot n, n^{-1} \cdot n_{v j}, \ldots\right\rangle .
\end{aligned}
$$

We define the $\hat{A}_{v}^{g, n}$ operator above by its operation on a state vector $\left|g_{v}, n_{i v}, n_{v j}, \ldots\right\rangle$. Under the $\hat{A}_{v}^{g, n}$ operation, the group element assigned to $v$ as $\left|g_{v}\right\rangle$ has evolved to $v^{\prime}$ as $|g\rangle$, the link 
element assigned to iv as $\left|n_{i v}\right\rangle$ has evolved to $\left|n_{i v^{\prime}}\right\rangle=$ $\left|n_{i v} \cdot n\right\rangle$, and $\left|n_{v j}\right\rangle$ has evolved to $\left|n_{v^{\prime} j}\right\rangle=\left|n^{-1} \cdot n_{v j}\right\rangle$.

In any dimension, we can construct $(d-1)$-simplices (that can be of irregular sizes) as a lattice to fill the space.
More explicitly, consider, for example, a $2+1 \mathrm{D}$ SET; $\hat{A}_{v}^{g, n}$ acts on a Hilbert space state vector for a 2D spatial lattice system in Fig. 21, centered at the vertex $v$ and its six nearest-neighbor links:

$$
\begin{aligned}
\hat{A}_{v}^{g, n} & \left|g_{v}, g_{1}, g_{2}, g_{3}, g_{4}, g_{5}, g_{6} ; n_{v 1}, n_{v 2}, n_{3 v}, n_{4 v}, n_{5 v}, n_{v 6}\right\rangle \\
= & \frac{\mathcal{V}_{3}^{G, N}\left(g_{4}, g_{5}, g_{v}, g ; n_{45}, n_{5 v}, n\right) \mathcal{V}_{3}^{G, N}\left(g_{5}, g_{v}, g, g_{6} ; n_{5 v}, n, n^{-1} n_{v 6}\right) \mathcal{V}_{3}^{G, N}\left(g_{v}, g, g_{6}, g_{1} ; n, n^{-1} n_{v 6}, n_{61}\right)}{\mathcal{V}_{3}^{G, N}\left(g_{v}, g, g_{2}, g_{1} ; n, n^{-1} n_{v 2}, n_{21}\right) \mathcal{V}_{3}^{G, N}\left(g_{3}, g_{v}, g, g_{2} ; n_{3 v}, n, n^{-1} n_{v 2}\right) \mathcal{V}_{3}^{G, N}\left(g_{4}, g_{3}, g_{v}, g ; n_{43}, n_{3 v}, n\right)} \\
& \quad \times\left|g, g_{1}, g_{2}, g_{3}, g_{4}, g_{5}, g_{6} ; n^{-1} \cdot n_{v 1}, n^{-1} \cdot n_{v 2}, n_{3 v} \cdot n, n_{4 v} \cdot n, n_{5 v} \cdot n, n^{-1} \cdot n_{v 6}\right\rangle .
\end{aligned}
$$

We design the $\hat{B}_{f}$ term as the zero flux constraint on each face or plaquette. More explicitly, consider a face $f$ (in Fig. 21) with three vertices (assigned $g_{1}, g_{2}, g_{v}$ ) and three links (assigned $n_{v 2}, n_{21}, n_{v 1}$ ), the $B_{f}$ acts on the corresponding state vector $\left|g_{1}, g_{2}, g_{v} ; n_{v 2}, n_{21}, n_{v 1}\right\rangle$ as

$$
\begin{aligned}
& \hat{B}_{f}\left|g_{1}, g_{2}, g_{v} ; n_{v 2}, n_{21}, n_{v 1}\right\rangle \\
& \quad=\left(\delta_{n_{v 2} n_{21} n_{1 v}=1}\right) \cdot\left|g_{1}, g_{2}, g_{v} ; n_{v 2}, n_{21}, n_{v 1}\right\rangle .
\end{aligned}
$$

The $\delta_{n_{v 2}, n_{21} n_{1 v}=1}$ is a Kronecker delta, which gives 1 if $n_{v 2}, n_{21} n_{1 v}=1$ is trivial in $N$; thus, the flux through the face $f$ is zero. The $\delta_{n_{v 2}, n_{21} n_{1 v}=1}$ gives 0 otherwise. Even for SETs, the explicit zero flux condition is reduced to

$$
\left(g_{v}^{-1} n_{v 2} g_{2}\right)\left(g_{2}^{-1} n_{21} g_{1}\right)\left(g_{1}^{-1} n_{1 v} g_{v}\right)=n_{v 2} n_{21} n_{21}=1,
$$

the same as in the pure $N$-gauge theory of topological order. For SPTs with a nontrivial $G$ but a trivial $N=1$, the zero flux always manifests, and $\left(g_{v}^{-1} g_{2}\right)\left(g_{2}^{-1} g_{1}\right)\left(g_{1}^{-1} g_{v}\right)=1$. Some more remarks on the system are given as follows:

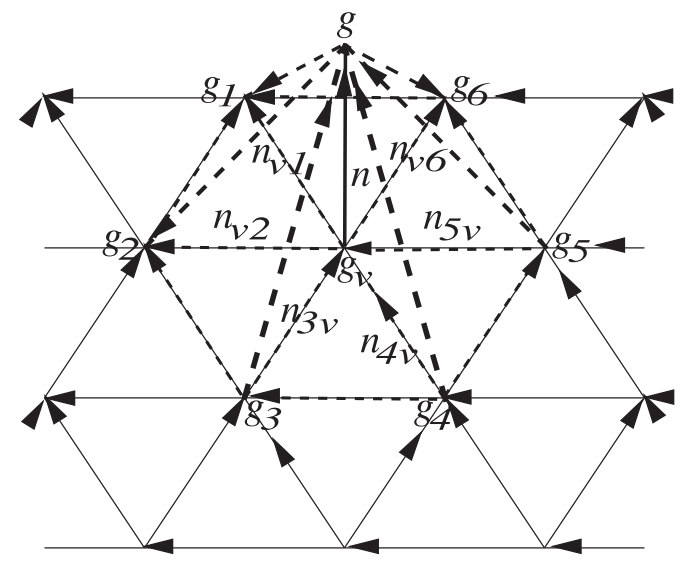

FIG. 21. The effective expression of $\hat{A}_{v}^{g, n}$ operation. Here, we show that $\hat{A}_{v}^{g, n}$ acts on a 2D spatial lattice on a site $v$ and its neighbor links. The explicit form is given in Eq. (9.24). The volume enclosed by dashed links contributes an amplitude filled by cocycles $\mathcal{V}^{G, N}$. A more general expression for any dimension is given in Eq. (9.23).
(1) All $\hat{A}_{v}^{g, n}$ and $\hat{B}_{f}$ have mutually commuting and selfcommuting nice properties. In principle, our model is an exactly soluble lattice model.

(2) Since the SPT always satisfies the zero flux on every face $f$, we can simplify the Hamiltonian without the $\hat{B}_{f}$ term: $\hat{H}_{\mathrm{SPT}}=-\sum_{v} \hat{A}_{v}$. The additional $\hat{B}_{f}$ term in Eq. (9.21) for SETs and topological orders imposes the zero flux constraint at low energy. However, at high energy, at the cost of an energy penalty, the zero flux condition does not hold at those faces $f$ with energetic anyon excitations. The anyon excitations are created at the end points of extended operators (e.g., line operators in $2+1 D$ ). See also Remark 8.

(3) Hilbert space: The Hilbert space on each site $j$ is $\mathcal{H}_{j}$ with a Hilbert space dimension $|G|$ spanned by $\left|g_{j}\right\rangle$ for $g_{j} \in G$. The Hilbert space on each edge $i j$ is $\mathcal{H}_{i j}$ with a Hilbert space dimension $|N|$ spanned by $\left|n_{i j}\right\rangle$ for $n_{i j} \in N$. For our lattice Hamiltonian in Eq. (9.21), the total Hilbert space is a tensor product structure:

$$
\mathcal{H}_{\text {total }}=\otimes_{j} \mathcal{H}_{j} \otimes_{i j} \mathcal{H}_{i j}
$$

When we limit to a symmetric $G$-SPT, with $N=1$, we have a tensor product $\mathcal{H}_{\text {total }}=\otimes_{j} \mathcal{H}_{j}$ defined on sites. When we limit to a gauge group $N$-topological order, with $G=1$, we have a tensor product $\mathcal{H}_{\text {total }}=$ $\otimes_{i j} \mathcal{H}_{i j}$ defined on links. Naively, one may ask, "Is it not that the discrete gauge theory description of topological order has no tensor product Hilbert space $\mathcal{H}_{\text {total }} \neq \otimes_{i j} \mathcal{H}_{i j}$ ?" The answer is that the gauge theory description of topological order for our Hamiltonian in Eq. (9.21) only occurs at the lowestenergy ground states, when $\hat{B}_{f}=1$ as zero flux on every face. For those ground states of topological order, indeed, the Hilbert space is not a tensor product, $\mathcal{H}_{\text {total }} \neq \bigotimes_{i j} \mathcal{H}_{i j}$, due to the requirement of projection constrained by $\hat{B}_{f}=1$. Thus, our Hamiltonian as a local bosonic lattice model at 
higher energy contains more than a discrete gauge theory. The same argument holds for SET states.

(4) Gauge and global symmetries for Hamiltonians: The Hamiltonian in Eq. (9.21) is apparently invariant under the $N$-gauge [Eq. (9.7)] and $G$-global symmetry [Eq. (9.8)] transformations. For SETs and SPTs, each individual of $\hat{A}_{v}^{g, n}$ and $\hat{B}_{f}$ terms is both $N$-gauge invariant and $G$-global invariant. On the other hand, for a topological order of gauge group $N$ without any global symmetry (i.e., $G=1$ ), the individual $\hat{A}_{v}^{n}$ is not gauge invariant. For example, under a local gauge transformation $\mathbf{n}_{v}$ applied on the vertex $v$, it transforms $\hat{A}_{v}^{n} \rightarrow \hat{A}_{v}^{\left(\mathbf{n}_{v}\right) \cdot n}$. If a local gauge transformation is applied on a neighbored vertex next to $v$, then $\hat{A}_{v}^{n}$ is invariant. However, the overall $\hat{A}_{v}=\frac{1}{|N|} \sum_{\left[v v^{\prime}\right]=n \in N} \hat{A}_{v}^{n}$ is gauge invariant.

(5) Gauge and global symmetries for wave functions: For the SET state vector $|\Phi\rangle$ of Eq. (9.20), we can apply symmetry transformations on either the wave function coefficient $\Phi\left(\left\{g_{i}, n_{i j}\right\}_{M}\right)$ or on the basis $\left|\left\{g_{i}, n_{i j}\right\}_{M}\right\rangle$; the two transformations are equivalent by an inverse transformation on another. Thus, we focus on the transformations on the wave function $\Phi\left(\left\{g_{i}, n_{i j}\right\}_{M}\right)$.

(i) If $G$ is nontrivial, then we have either SPTs or SETs. It is easy to check that the cocycle $\mathcal{V}^{G, N}$ is both gauge and global symmetry invariant under $N$-gauge [Eq. (9.7)] and $G$-global symmetry [Eq. (9.8)] transformations. Thus, apparently, the wave function

$$
\begin{aligned}
\Phi\left(\left\{g_{i}, n_{i j}\right\}_{M}\right) & =\Phi\left[\left\{\left(\mathbf{n}_{i}\right) g_{i},\left(\mathbf{n}_{i}\right) n_{i j}\left(\mathbf{n}_{j}\right)^{-1}\right\}_{M}\right] \\
& =\Phi\left[\left\{(\mathbf{g}) g_{i},(\mathbf{g}) n_{i j}(\mathbf{g})^{-1}\right\}_{M}\right]
\end{aligned}
$$

is gauge and global-symmetry invariant under transformations of Eqs. (9.7) and (9.8).

(ii) If $G=1$ is trivial and the gauge group $N$ is nontrivial, then we have a pure gauge theory with topological order. The reduced inhomogeneous cocycle $\mathcal{V}^{G, N}=\omega^{N}$ alone is not gauge invariant; the wave function $\Phi\left(\left\{n_{i j}\right\}_{M}\right)$ is not gauge invariant, either. Even the ground state vector $|\Phi\rangle \propto \sum_{\left\{n_{i j}\right\}_{M}} \Phi\left(\left\{n_{i j}\right\}_{M}\right)\left|\left\{n_{i j}\right\}_{M}\right\rangle$ is not gauge invariant and is not gauge invariant up to a $U(1)$ phase. Namely, each wave function obtains a different $U(1)$ phase $e^{\mathrm{i} \theta\left(\left\{n_{i j}\right\}_{M}, \mathbf{n}_{i}\right)}$ that depends on the input $\left\{n_{i j}\right\}_{M}$ and gauge transformation $\mathbf{n}_{i}$, i.e., $\Phi\left(\left\{n_{i j}\right\}_{M}\right) \rightarrow e^{\mathrm{i} \theta\left(\left\{n_{i j}\right\}_{M}, \mathbf{n}_{i}\right)} \Phi\left(\left\{n_{i j}\right\}_{M}\right)$. We define such a gauge-transformed state vector as $|\Phi\rangle \rightarrow\left|\Phi\left(\mathbf{n}_{i}\right)\right\rangle$. However, as long as any physical observable $\langle\hat{O}\rangle=\langle\Phi|\hat{O}| \Phi\rangle$ is strictly gauge invariant as we show below [73], the theory is well defined. We find that $\langle\hat{O}\rangle$ is indeed gauge invariant,

$$
\begin{aligned}
\langle\Phi|\hat{O}| \Phi\rangle & =\sum_{\left\{n_{i j}\right\}} \sum_{\left\{\tilde{n}_{i j}\right\}} \Phi^{\dagger}\left(\left\{n_{i j}\right\}_{M}\right) c_{\left\{n_{i j}\right\}}^{\left\{\tilde{n}_{i j}\right\}} \Phi\left(\left\{\tilde{n}_{i j}\right\}_{M}\right) \\
& =\left\langle\Phi\left(\mathbf{n}_{i}\right)|\hat{O}| \Phi\left(\mathbf{n}_{i}\right)\right\rangle,
\end{aligned}
$$

where we have considered a generic operator $\hat{O}$ defined by its operation on $|\Phi\rangle$ :

$$
\begin{aligned}
\hat{O}|\Phi\rangle & =\hat{O} \sum_{\left\{n_{i j}\right\}} \Phi\left(\left\{n_{i j}\right\}_{M}\right)\left|\left\{n_{i j}\right\}_{M}\right\rangle \\
& =\sum_{\left\{n_{i j}\right\}} \sum_{\left\{\tilde{n}_{i j}\right\}} c_{\left\{n_{i j}\right\}}^{\left\{\tilde{n}_{i j}\right\}} \Phi\left(\left\{\tilde{n}_{i j}\right\}_{M}\right)\left|\left\{n_{i j}\right\}_{M}\right\rangle
\end{aligned}
$$

with generic $c_{\left\{n_{i j}\right\}}^{\left\{\tilde{n}_{i j}\right\}}$ coefficients.

(6) Wave functions and their independence of input $g^{*}$ and $n_{i_{0} i_{*}}$ : Consider a wave function on a closed space $M$ defined in Eq. (9.19).

(i) The SPT wave function $\Phi\left(\left\{g_{i}\right\}_{M}\right)_{\mathrm{SPT}}$ is independent of the input choice $g^{*}$. Namely, changing $g^{*}$ to $g^{* *} \equiv(\mathbf{g})^{-1} g^{*}$,

$$
\begin{aligned}
& \Phi\left(\left\{g_{i}\right\}_{M}\right)_{\mathrm{SPT}}=\prod_{\{\ldots\}} \nu_{d}^{G s_{i_{0} \ldots i_{*}}}\left(g_{i_{0}}, \ldots, g_{i_{d-1}}, g^{*}\right) \\
& =\prod_{\{\ldots\}} \nu_{d}^{G s_{\ldots}}\left[(\mathbf{g}) g_{i_{0}}, \ldots,(\mathbf{g}) g_{i_{d-1}}, g^{*}\right] \\
& =\prod_{\{\ldots\}} \nu_{d}^{G s}\left[g_{i_{0}}, \ldots, g_{i_{d-1}},(\mathbf{g})^{-1} g^{*}\right] \\
& =\prod_{\{\ldots\}} \nu_{d}^{G s_{\ldots}}\left(g_{i_{0}}, \ldots, g_{i_{d-1}}, g^{*}\right) .
\end{aligned}
$$

Here, we use the fact that $\Phi\left(\left\{g_{i}\right\}_{M}\right)_{\mathrm{SPT}}$ is $G$-global symmetry invariant in the second equality. This proof, $\left[\left(\Phi\left[\left\{(\mathbf{g}) g_{i}\right\}_{M}\right]_{\mathrm{SPT}}\right) /\right.$ $\left.\left(\Phi\left(\left\{g_{i}\right\}_{M}\right)_{\mathrm{SPT}}\right)\right]=1$, requires the use of a $G-$ cocycle condition, and we will show a complete proof in Sec. IX B 4, even in the presence of a gapped boundary or interface. We also use that $\nu_{d}^{G}\left(\left\{g_{i}\right\}\right)=\nu_{d}^{G}\left(\left\{(\mathbf{g})^{-1} g_{i}\right\}\right)$ due to the property of a homogeneous cocycle in the third equality. One quick way to visualize this proof [Eq. (9.29)] is that the ratio $\left[\left(\Phi\left[\left\{(\mathbf{g}) g_{i}\right\}_{M}\right]_{\mathrm{SPT}}\right) /\right.$ $\left.\left(\Phi\left(\left\{g_{i}\right\}_{M}\right)_{\mathrm{SPT}}\right)\right]$ yields a term equivalent to a product of coboundary terms; fortunately, the overall coboundary terms on a closed space $M$ must cancel out to be 1 . 
(ii) The topological order and SET wave function $\Phi\left(\left\{g_{i}, n_{i j}\right\}_{M}\right)_{\text {SET }}$ can be defined in such a way that it is independent of the input $g^{*}$ and $n_{i_{0} i_{*}}$. It is easier to prove that if we design and evaluate Eq. (9.19) in terms of homogeneous $G$ cocycles.
Below, we show that replacing $g^{*} \rightarrow g^{\prime *} \equiv$ $(\mathbf{g})^{-1} g^{*}$ and $n_{i_{*} i_{0}} \rightarrow n_{i_{*} i_{0}}^{\prime} \equiv(\mathbf{n}) n_{i_{*} i_{0}}$, with a slight reordering of vertex indices and branch structure for our convenience, the $\Phi\left(\left\{g_{i}, n_{i j}\right\}_{M}\right)_{\mathrm{SET}}$ is still invariant:

$$
\begin{aligned}
& \Phi\left(\left\{g_{i}, n_{i j}\right\}_{M}\right)_{\mathrm{SET}}=\prod_{\{\ldots\}} \nu_{d}^{G s_{i_{*} \ldots i_{d-1}}}\left(g^{*}, n_{i_{*} i_{0}} g_{i_{0}}, n_{i_{*} i_{0}} n_{i_{0} i_{1}} g_{i_{1}}, \ldots, n_{i_{*} i_{0}} n_{i_{0} i_{1}} \ldots n_{i_{d-2} i_{d-1}} g_{i_{d-1}}\right) \\
& =\prod_{\{\ldots\}} \nu_{d}^{G s}\left[g^{*},(\mathbf{g}) n_{i_{*} i_{0}} g_{i_{0}},(\mathbf{g}) n_{i_{*} i_{0}} n_{i_{0} i_{1}} g_{i_{1}}, \ldots,(\mathbf{g}) n_{i_{*} i_{0}} n_{i_{0} i_{1}} \ldots n_{i_{d-2} i_{d-1}} g_{i_{d-1}}\right] \\
& =\left.\prod_{\{\ldots\}} \nu_{d}^{G s} \ldots\left(g^{\prime *}, n_{i_{*} i_{0}} g_{i_{0}}, n_{i_{*} i_{0}} n_{i_{0} i_{1}} g_{i_{1}}, \ldots, n_{i_{*} i_{0}} n_{i_{0} i_{1}} \ldots n_{i_{d-2} i_{d-1}} g_{i_{d-1}}\right)\right|_{g^{* *} \equiv(\mathbf{g})^{-1} g^{*}} . \\
& \Phi\left(\left\{g_{i}, n_{i j}\right\}_{M}\right)_{\mathrm{SET}}=\prod_{\{\ldots\}} \nu_{d}^{G s_{i_{*} \ldots i_{d-1}}}\left(g^{*}, n_{i_{*} i_{0}} g_{i_{0}}, n_{i_{*} i_{0}} n_{i_{0} i_{1}} g_{i_{1}}, \ldots, n_{i_{*} i_{0}} n_{i_{0} i_{1}} \ldots n_{i_{d-2} i_{d-1}} g_{i_{d-1}}\right) \\
& =\prod_{\{\ldots\}} \nu_{d}^{G s_{\ldots}}\left[g^{*},(\mathbf{n}) n_{i_{*} i_{0}} g_{i_{0}},(\mathbf{n}) n_{i_{*} i_{0}} n_{i_{0} i_{1}} g_{i_{1}}, \ldots,(\mathbf{n}) n_{i_{*} i_{0}} n_{i_{0} i_{1}} \ldots n_{i_{d-2} i_{d-1}} g_{i_{d-1}}\right] \\
& =\left.\prod_{\{\ldots\}} \nu_{d}^{G S} \ldots\left(g^{*}, n_{i_{*} i_{0}}^{\prime} g_{i_{0}}, n_{i_{*} i_{0}}^{\prime} n_{i_{0} i_{1}} g_{i_{1}}, \ldots, n_{i_{*} i_{0}}^{\prime} n_{i_{0} i_{1}} \ldots n_{i_{d-2} i_{d-1}} g_{i_{d-1}}\right)\right|_{n_{i_{*} i_{0}}^{\prime}} \equiv(\mathbf{n}) n_{i_{*} i_{0}} .
\end{aligned}
$$

The $\Phi\left(\left\{g_{i}, n_{i j}\right\}_{M}\right)_{\text {SET }}$ becomes that of topological order $\Phi\left(\left\{n_{i j}\right\}_{M}\right)_{\text {TO }}$ if we set all $g=1$ for the trivial $G$. The proofs in Eqs. (9.30) and (9.31) again require the use of a $G$-cocycle condition and the property of a homogeneous cocycle.

(7) Local unitary transformation and the Hamiltonian: We can define a unitary transformation $\hat{U}$ as

$$
\begin{aligned}
\hat{U}= & \sum_{\left\{g_{i}, n_{i j}\right\}_{M}} \prod_{\{\ldots\}} \mathcal{V}_{d}^{G, N}\left(g_{i_{0}}, \ldots, g^{*} ; n_{i_{0} i_{1}}, n_{i_{1} i_{2}}, \ldots, n_{i_{d-1} i_{*}}\right) \\
& \times\left|\left\{g_{i}, n_{i j}\right\}_{M}\right\rangle\left\langle\left\{g_{i}, n_{i j}\right\}_{M}\right| .
\end{aligned}
$$

We can view that the above $\mathcal{V}_{d}^{G, N}$ is a $U(1)$ complex phase determined by local input data $\left\{g_{i_{0}}, \cdots\right.$; $\left.n_{i_{0} i_{1}}, \cdots\right\}$ that are given within a local $(d-1)$ simplex. Since the $\hat{U}$ sends the input state $\left|\left\{g_{i}, n_{i j}\right\}_{M}\right\rangle$ to the same output state, the overall $U(1)$ phase is determined by $\prod_{\{\ldots\}} \mathcal{V}_{d}^{G, N}$, which is a product of $U(1)$ phases assigned to each $(d-1)$ simplex.

(i) For SPTs, it is

$$
\hat{U}=\sum_{\left\{g_{i}\right\}_{M}} \prod_{\{\ldots\}} \nu_{d}^{G}\left(g_{i}, \ldots, g^{*}\right)\left|\left\{g_{i}\right\}_{M}\right\rangle\left\langle\left\{g_{i}\right\}_{M}\right| .
$$

For SPTs, actually, this $\hat{U}$ is a local unitary transformation (LUT), because this $\hat{U}$ is formed by a local circuit of many independent $\nu_{d+1}$ on each local simplex. Overall, $\hat{U}$ is a unitary diagonal matrix acting on the full Hilbert space with diagonal elements assigned with distinct $U(1)$ phases. Under this LCT, the SPT $|\Phi\rangle$ is deformed to $U^{\dagger}|\Phi\rangle=\left|\Phi_{0}\right\rangle$ of Eq. (9.17) as a trivial product state. However, such a LCT locally breaks the global $G$-symmetry of SPTs, because each $\nu_{d}^{G}\left(g \cdot g_{i}, \ldots, g^{*}\right)$ is not $g$-invariant with a fixed $g^{*}$. The LUT can deform such a short-range entangled state of SPTs to a trivial product state, at the cost of breaking its global $G$-symmetry.

The SPT Hamiltonian (without the $\hat{B}_{f}$ term) can be rewritten as

$$
\begin{aligned}
\hat{H} & =\sum_{j} \hat{U} \hat{H}_{j} \hat{U}^{\dagger}=-\sum_{j} \hat{U}\left|\phi_{j}\right\rangle\left\langle\phi_{j}\right| \hat{U}^{\dagger} \\
& =\sum_{j} \hat{U}\left(-\sum_{g_{j}, g_{j}^{\prime} \in G}\left|g_{j}\right\rangle\left\langle g_{j}^{\prime}\right|\right) \hat{U}^{\dagger} .
\end{aligned}
$$

The $\left|\phi_{j}\right\rangle=\sum_{g_{j} \in G}\left|g_{j}\right\rangle$ is an equal-weight sum of all states for all $g_{j}$ on each site.

(ii) For topological orders or SETs, the $\hat{U}$ defined in Eq. (9.32) is not unitary for the total Hilbert space $\mathcal{H}_{\text {total }}=\otimes_{j} \mathcal{H}_{j} \otimes_{i j} \mathcal{H}_{i j}$, because $\mathcal{V}_{d}^{G, N}\left(n_{12}, n_{23}, \ldots\right)$ is defined to be 0 when a 
closed loop $n_{12} n_{23} n_{31} \neq 1$. We can artificially redefine $\hat{U}^{\prime}$ to design those zero $\mathcal{V}_{d}^{G, N}$ terms to be 1 by hand, and make $\hat{U}^{\prime}$ a new unitary matrix. For example, one such unitary deformation sends to

$$
\begin{aligned}
U^{\prime \dagger}|\Phi\rangle & =\mathrm{P}\left[\sum_{\left\{g_{i}, n_{i j}\right\}_{M}}\left|\left\{g_{i}, n_{i j}\right\}_{M}\right\rangle\right] \\
& =\mathrm{P}\left[\otimes_{i}\left(\sum_{g_{i}}\left|g_{i}\right\rangle\right) \bigotimes_{i j}\left(\sum_{n_{i j}}\left|n_{i j}\right\rangle\right)\right],
\end{aligned}
$$

where $\mathrm{P}$ is a projection operator imposing the zero flux condition through a closed loop as $n_{12} n_{23} n_{31}=1$, and $\mathrm{P}$ projects out any $n_{12} n_{23} n_{31} \neq 1$ state. However, this final state is very different from a trivial product state, e.g., $\otimes_{i}\left(\sum_{g_{i}}\left|g_{i}\right\rangle\right) \bigotimes_{i j}\left(\sum_{n_{i j}}\left|n_{i j}\right\rangle\right)$. Regardless of how we design a unitary $\hat{U}^{\prime}$ matrix, we cannot deform the ground state $|\Phi\rangle$ of topological orders or SETs to a trivial product state through any local unitary transformation. This reason is due to superposed extended loop states, as ground states of intrinsic topological orders are highly longrange entangled - their information encoded in the projection $\mathrm{P}$ on the zero flux condition is incompatible with a trivial product state. The LUT cannot deform a long-range entangled state to a trivial product state. Thus, the topological orders or SET Hamiltonian cannot be rewritten as $\hat{H}=\sum_{j} \hat{U}^{\prime} \hat{H}_{j} \hat{U}^{\prime \dagger}$, for any unitary $\hat{U}^{\prime}$ and for some local Hamiltonian $\sum_{j} \hat{H}_{j}$ whose ground state is a trivial product state.

(8) Degenerate ground states with holonomies around noncontractible cycles: So far, we have focused only on a ground state $|\Phi\rangle$ that has no holonomies around noncontractible cycles and that can be deformed to a trivial product state. However, for gauge theories of topological orders and SETs, we have distinct degenerate ground states when the spatial topology is nontrivial (e.g., a 2D spatial torus $T_{x y}^{2}$ ). Starting from $|\Phi\rangle$, we can generate other degenerate ground states by inserting extended operators as holonomies around noncontractible cycles. Without losing generality, let us consider a $2+1 \mathrm{D}$ system; we have generic line operators $\hat{W}_{\mathcal{U}}^{S^{1}}$ in a 2D spatial torus $T_{x y}^{2}$ with coordinates $x$ and $y$. We can fully generate distinct ground states spanning the dimensions of Hilbert space on $T_{x y}^{2}$ by

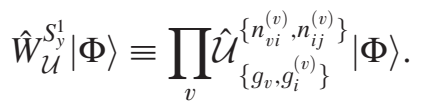

Here, $S_{y}^{1}$ in $\hat{W}_{\mathcal{U}}^{S_{y}^{1}}$ means that the line operator has a cycle around $S_{y}^{1}$, so the $\prod_{v}$ means a series of vertices $v$ spanning around the $S_{y}^{1}$-cycle, for

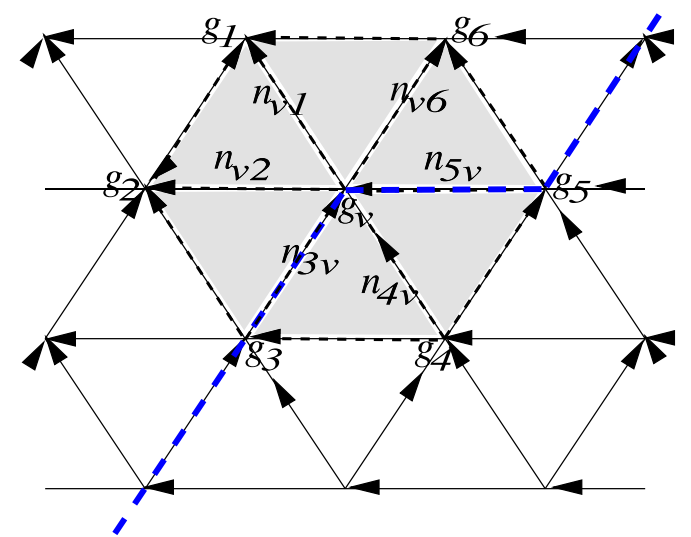

FIG. 22. An example of line operator $\hat{W}_{\mathcal{U}}^{S_{y}^{1}} \equiv \prod_{v} \hat{\mathcal{U}}_{\left\{g_{v}, g_{i}^{(j)}\right\}}^{\left\{n_{v i}^{(v)}, n_{i j}^{(v)}\right\}}$ acts along the blue dashed line. The product of $v$ spans along all the vertices on the blue dashed line. One of the most generic operators $\hat{\mathcal{U}}_{\left\{g_{v}, g_{i}^{(v)}\right\}}^{\left\{n_{v i}^{(v)}, n_{i j}^{(v)}\right\}}$ on this lattice centered at a vertex $v$ acts on a local Hilbert space of $7 \mathrm{G}$-vertices and $12 \mathrm{~N}$-links on a shaded honeycomb region; thus, it acts on a Hilbert space of dimensions $|G|^{7}|N|^{12}$.

example, along the blue dashed line in Fig. 22. The $\hat{\mathcal{U}}_{\left\{g_{v}, g_{i}^{(v)}\right\}}^{\left\{n_{v i}^{(v)}, n_{i j}^{(v)}\right\}}$ is a shorthand expression for $\hat{\mathcal{U}}_{g_{v 1}, n_{v 2}^{(v)}, n_{3 v}^{(v)}, n_{v v}^{(v)}, n_{5 v}^{(v)}, n_{v 6}^{(v)}, n_{21}^{(v)}, n_{32}^{(v)}, n_{43}^{(v)}, n_{45}^{(v)}, n_{56}^{(v)}, n_{61}^{(v)}, g_{4}^{(v)}, g_{5}^{(v)}, g_{6}^{(v)}}^{n^{(v)}}$, which acts on the honeycomb shaded region in Fig. 22. Examples of $\hat{\mathcal{U}}_{\left\{g_{v i}, g_{i}^{(v)}\right\}}^{\left\{n_{v i}^{(v)}, n_{i j}^{(v)}\right\}}$ include the $\hat{A}_{v}^{g, n}$ and $\hat{B}_{f}$ terms. For example, for a $Z_{2}$ toric code [74] on a $T^{2}$ torus, the expression for degenerate ground states $\hat{W}_{\mathcal{U}}^{S_{y}^{1}}|\Phi\rangle$ boils down to

$$
\left(\prod \sigma_{z}\right)^{q}\left(\prod \sigma_{x}\right)^{m}|\Phi\rangle,
$$

where $\sigma_{x}$ and $\sigma_{z}$ are the rank-2 Pauli matrices. The product $\prod$ is along the $S_{y}^{1}$ line operator. The $(q, m)$ are integer mod 2 values, and $(q, m)=(0,0)$, $(1,0),(0,1),(1,1)$ are four distinct ground states. Moreover, a generic $\hat{\mathcal{U}}_{\left\{g_{v}, g_{i}^{(v)}\right\}}^{\left\{n_{v i}^{(v)}, n_{i j}^{(v)}\right\}}$ does not need to commute with $\hat{A}_{v}^{g, n}$ and $\hat{B}_{f}$, and it can violate the zero flux condition of Remark 2. Thus, such a $\hat{\mathcal{U}}_{\left\{g_{v}, g_{i}^{(v)}\right\}}^{\left\{n_{v i}^{(v)}, n_{i j}^{(v)}\right\}}$ can create anyon excitations that cost higher energy.

We can easily generalize the above discussion $(2+1 \mathrm{D})$ to any spacetime dimension.

\section{Anomalous symmetry-preserving gapped boundary or interface of bulk SPTs and SETs}

Continued from Sec. IX B 2, we develop further to formulate a lattice wave function and Hamiltonian for 
topological phases with gapped boundaries or interfaces. We first focus on a bulk $G$-SPT on an open manifold, while the gapped boundary has an anomalous $H$-SPT that cannot exist without an extended bulk, via a group extension $H / K=G$, in Sec. IX A 4. Along the way, we comment on how to easily generalize to a bulk with SETs.

(i) Wave function: For the wave function, we can simply adopt the $G$-SPT limit of Eq. (9.19) as $\Phi\left(\left\{g_{i}\right\}_{M}\right) \equiv \prod_{\{\ldots\}} \nu_{d}^{G s_{i_{0} \ldots i_{*}}}\left(g_{i_{0}}, \ldots, g^{*}\right)$, defined first on a closed space $M \equiv M^{d-1}$ of $(d-1)$-spatial dimensions. The $g^{*}$ is again some fixed value outside the $M^{d-1}$. We would like to keep the degrees of freedom on each site with Hilbert space dimensions $|G|$ on the gapped left region $R_{\mathrm{I}}$ and extend the site's Hilbert space dimensions to $|H|$ on the gapped right region $R_{\mathrm{II}}$, as well as on the interface $\partial R$ (三 $\partial R_{\mathrm{I}} \equiv \partial R_{\mathrm{II}}$ up to an orientation). We denote the group element in $H$ assigned along $\partial R$ as $h^{\partial} \in H$. We also extend the Hilbert space dimensions of $i_{*}$ from $|G|$ to $|H|$, and we choose $r\left(h^{*}\right)=g^{*}$. The modified wave function defined on $M=R_{\mathrm{I}} \cup R_{\mathrm{II}}$ is

$$
\begin{aligned}
& \Phi\left(\left\{g_{i}, h_{j}\right\}\right) \equiv \Phi\left(\left\{g_{i}\right\}_{R_{\mathrm{I}}},\left\{h_{j}^{\partial}\right\}_{\partial R},\left\{h_{j}\right\}_{R_{\mathrm{II}}}\right) \\
& =\prod_{\{\ldots\}} \nu_{d}^{G s_{i_{a} \ldots i_{*}}}\left[\left\{g_{i_{a}}\right\}_{R_{\mathrm{I}}}, r\left(h^{*}\right)\right] \cdot \prod_{\{\ldots\}} \nu_{d}^{G s_{i_{a j} \ldots j_{b}}}\left[\left\{g_{i_{a}}\right\}_{R_{\mathrm{I}}},\left\{r\left(h_{j_{b}}^{\partial}\right)\right\}_{\partial R}, r\left(h^{*}\right)\right] \\
& \cdot \prod_{\{\ldots\}} \nu_{d}^{G s_{j a j_{b} \ldots i_{*}}}\left[\left\{r\left(h_{j_{a}}^{\partial}\right)\right\}_{\partial R},\left\{r\left(h_{j_{b}}\right)\right\}_{R_{\mathrm{II}}}, r\left(h^{*}\right)\right] \\
& =\left(\prod_{\{\ldots\}} \nu_{d}^{G s_{i_{a} \ldots i_{*}}}\left[\left\{g_{i_{a}}\right\}_{R_{\mathrm{I}}}, r\left(h^{*}\right)\right] \cdot \prod_{\{\ldots\}} \nu_{d}^{G s_{i_{a j} j_{b} \ldots i_{*}}}\left[\left\{g_{i_{a}}\right\}_{R_{\mathrm{I}}},\left\{r\left(h_{j_{b}}^{\partial}\right)\right\}_{\partial R}, r\left(h^{*}\right)\right]\right) \\
& \cdot\left(\prod_{\{\ldots\}} \mu_{d-1}^{H s_{j_{a} \ldots i_{*}}}\left[\left\{h_{j_{a}}^{\partial}\right\}_{\partial R}, r\left(h^{*}\right)\right]\right)\left(\prod_{\{\ldots\}} \mu_{d-1}^{H s_{j j_{b} \ldots i_{*}}}\left(\left\{h_{j_{a}}^{\partial}\right\}_{\partial R},\left\{h_{j_{b}}\right\}_{R_{\mathrm{II}}}\right)\right) \\
& \equiv \Phi_{R_{\mathrm{I}}}\left(\left\{g_{i}\right\},\left\{h_{j}^{\partial}\right\}\right) \Phi_{\partial R}\left(\left\{h_{j}^{\partial}\right\}\right) \Phi_{R_{\mathrm{II}}}\left(\left\{h_{j}^{\partial}\right\},\left\{h_{j}\right\}\right) . \\
& \underset{\operatorname{LUT}}{\longrightarrow}\left(\prod_{\{\ldots\}} \nu_{d}^{G s_{i_{a} \ldots i_{*}}}\left[\left\{g_{i_{a}}\right\}_{R_{\mathrm{I}}}, r\left(h^{*}\right)\right] \cdot \prod_{\{\ldots\}} \nu_{d}^{G s_{a_{a j} j_{b} \ldots i_{*}}}\left[\left\{g_{i_{a}}\right\}_{R_{\mathrm{I}}},\left\{r\left(h_{j_{b}}^{\partial}\right)\right\}_{\partial R}, r\left(h^{*}\right)\right]\right) \cdot\left(\prod_{\{\ldots\}} \mu_{d-1}^{H s_{j_{a} \ldots i_{*}}}\left[\left\{h_{j_{a}}^{\partial}\right\}_{\partial R}, r\left(h^{*}\right)\right]\right) \\
& \equiv \Phi_{R_{\mathrm{I}}}\left(\left\{g_{i}\right\},\left\{h_{j}^{\partial}\right\}\right) \Phi_{\partial R}\left(\left\{h_{j}^{\partial}\right\}\right),
\end{aligned}
$$

where we have split the above $H$-coboundary $\nu_{d}^{G}[r(h)]=\nu_{d}^{H}(h)$ in Eq. (9.36) into $H$-cochains $\mu_{d-1}^{H}$ in Eq. (9.37). We define

$$
\begin{aligned}
\Phi_{R_{\mathrm{I}}}\left(\left\{g_{i}\right\},\left\{h_{j}^{\partial}\right\}\right) & \equiv\left(\prod_{\{\ldots\}} \nu_{d}^{G s_{i_{a} \ldots i_{*}}}\left[\left\{g_{i_{a}}\right\}_{R_{\mathrm{I}}}, r\left(h^{*}\right)\right] \cdot \prod_{\{\ldots\}} \nu_{d}^{G s_{i_{a} j_{b} \ldots i_{*}}}\left[\left\{g_{i_{a}}\right\}_{R_{\mathrm{I}}},\left\{r\left(h_{j_{b}}^{\partial}\right)\right\}_{\partial R}, r\left(h^{*}\right)\right]\right), \\
\Phi_{\partial R}\left(\left\{h_{j}^{\partial}\right\}\right) & \equiv\left(\prod_{\{\ldots\}} \mu_{d-1}^{H s_{j_{a} \ldots i_{*}}}\left[\left\{h_{j_{a}}^{\partial}\right\}_{\partial R}, r\left(h^{*}\right)\right]\right), \\
\Phi_{R_{\mathrm{II}}}\left(\left\{h_{j}^{\partial}\right\},\left\{h_{j}\right\}\right) & \equiv\left(\prod_{\{\ldots\}} \mu_{d-1}^{H s_{j_{a j} \ldots i_{*}}}\left(\left\{h_{j_{a}}^{\partial}\right\}_{\partial R},\left\{h_{j_{b}}\right\}_{R_{\mathrm{II}}}\right)\right) .
\end{aligned}
$$

Notice that $\Phi_{R_{\mathrm{II}}}\left(\left\{h_{j}^{\partial}\right\},\left\{h_{j}\right\}\right)$ is simplified to no dependence on $h^{*}$ because those $\mu_{d-1}^{H}$ that depend on $h^{*}$ are pair canceled out due to overlapping on the same $(d-1)$-simplex with opposite orientations \pm 1 . From Eq. (9.38) to Eq. (9.39), the notation "LUT" means that we do a local unitary transformation (LUT) to deform $\Phi_{R_{\mathrm{II}}}$ to a gapped trivial product state $\Phi_{R_{\mathrm{II}}}=1$ without breaking any symmetry. Thus, the simplified nontrivial wave function only resides on $R_{\mathrm{I}}$ and $\partial R$ as $\Phi\left(\left\{g_{i}, h_{j}\right\}\right) \equiv \Phi_{R_{\mathrm{I}}}\left(\left\{g_{i}\right\},\left\{h_{j}^{\partial}\right\}\right) \Phi_{\partial R}\left(\left\{h_{j}^{\partial}\right\}\right)$. 
For example, more explicitly in $2+1 \mathrm{D}$,

$$
\begin{aligned}
\Phi\left(\left\{g_{i}, h_{j}\right\}\right) \equiv & \Phi_{R_{\mathrm{I}}}\left(\left\{g_{i}\right\},\left\{h_{j}^{\partial}\right\}\right) \Phi_{\partial R}\left(\left\{h_{j}^{\partial}\right\}\right) \\
= & \prod_{\{\ldots\}} \nu_{3}^{G s}\left[g_{i_{1}}, g_{i_{2}}, g_{i_{3}}, r\left(h^{*}\right)\right] \nu_{3}^{G s}\left[r\left(h_{j_{1}}^{\partial}\right), g_{i_{2}}, g_{i_{3}}, r\left(h^{*}\right)\right] \nu_{3}^{G s}\left[r\left(h_{j_{1}}^{\partial}\right), r\left(h_{j_{2}}^{\partial}\right), g_{i_{3}}, r\left(h^{*}\right)\right] \\
& \times \mu_{2}^{H s}\left(h_{j_{1}}^{\partial}, h_{j_{2}}^{\partial}, h^{*}\right) \mu_{2}^{H s}\left(h_{j_{1}}^{\partial}, h_{j_{2}}^{\partial}, h_{j_{3}}\right) \mu_{2}^{H s}\left(h_{j_{1}}^{\partial}, h_{j_{3}}, h_{j_{4}}\right) \mu_{2}^{H s}\left(h_{j_{3}}, h_{j_{4}}, h_{j_{5}}\right) \\
& \stackrel{\mathrm{LUT}}{\longrightarrow} \prod_{\{\ldots\}} \nu_{3}^{G s}\left[g_{i_{1}}, g_{i_{2}}, g_{i_{3}}, r\left(h^{*}\right)\right] \nu_{3}^{G s}\left[r\left(h_{j_{1}}^{\partial}\right), g_{i_{2}}, g_{i_{3}}, r\left(h^{*}\right)\right] \nu_{3}^{G s}\left[r\left(h_{j_{1}}^{\partial}\right), r\left(h_{j_{2}}^{\partial}\right), g_{i_{3}}, r\left(h^{*}\right)\right] \cdot \prod_{\{\ldots\}} \mu_{2}^{H s}\left(h_{j_{1}}^{\partial}, h_{j_{2}}^{\partial}, h^{*}\right) .
\end{aligned}
$$

Here, the shorthand $s= \pm 1$ depends on the ordering of each assigned simplex. We see that those $\mu_{2}^{H}$ that do not depend on $h^{*}$ can be deformed to a gapped trivial product state by local unitary transformation without breaking any symmetry (again, we denote the procedure as "LUT"), because the homogeneous cochain satisfies $\mu_{d-1}^{H}\left(\left\{(\mathbf{h}) \cdot h_{j}\right\}\right)=$ $\mu_{d-1}^{H}\left(\left\{h_{j}\right\}\right)$. Thus, keeping only $\mu_{2}^{H}\left(h_{j_{1}}^{\partial}, h_{j_{2}}^{\partial}, h^{*}\right)$ but removing other $\mu_{2}^{H}$, we obtain the last simplified equality. In generic dimensions, we have Eq. (9.40). (ii) Lattice Hamiltonian: The Hamiltonian for the above gapped ground state has the same form in the bulk region $R$ as $\hat{H}=-\sum_{v} \hat{A}_{v}-\sum_{f} \hat{B}_{f}$ in Eq. (9.21). However, we need to modify the boundary term on $\partial R$. The first term $\hat{A}_{v}$ on the boundary acts on the wave function of a constant-time slice through each vertex $v$ in the space by lifting the initial state through an "imaginary time" evolution to a new state with a vertex $v^{\prime}$ via

$$
\begin{gathered}
\hat{A}_{v}=\frac{1}{|H|} \sum_{h \in H} \hat{A}_{v}^{h} . \\
\hat{A}_{v}^{h}\left|h_{v},\left\{h_{j}^{\partial}\right\},\left\{g_{i}\right\}\right\rangle=\prod_{\{\ldots\}} \nu_{d}^{G s}\left(r(h), r\left(h_{v}\right),\left\{r\left(h_{j}^{\partial}\right)\right\},\left\{g_{i}\right\}\right) \prod_{\{\ldots\}} \mu_{d-1}^{H s_{-1}}\left(h, h_{v},\left\{h_{j}^{\partial}\right\}\right)\left|h,\left\{h_{j}^{\partial}\right\},\left\{g_{i}\right\} \ldots\right\rangle .
\end{gathered}
$$

More specifically, the effective $2+1 \mathrm{D}$ Hamiltonian term along the $1+1 \mathrm{D}$ gapped boundary $\partial R$, shown in Fig. 23, is written as

$$
\hat{A}_{v}^{h}\left|h_{v}, h_{1}, g_{2}, g_{3}, h_{4}\right\rangle=\frac{\mu_{2}^{H}\left(h_{v}, h, h_{1}\right) \mu_{2}^{H}\left(h_{4}, h_{v}, h\right)}{\nu_{3}^{G}\left[r\left(h_{v}\right), r(h), g_{2}, r\left(h_{1}\right)\right] \nu_{3}^{G}\left[g_{3}, r\left(h_{v}\right), r(h), g_{2}\right] \nu_{3}^{G}\left[r\left(h_{4}\right), g_{3}, r\left(h_{v}\right), r(h)\right]}\left|h, h_{1}, g_{2}, g_{3}, h_{4}\right\rangle \text {. }
$$

The $\hat{B}_{f}$ term imposes trivial $G$ - and $H$-holonomies for the contractible loop. But here, $\hat{B}_{f}$ does not play any role for SPTs, because SPTs always have trivial holonomy regardless of whether the loop is contractible or not.

(iii) More generic bulk or gapped boundary SET wave function and Hamiltonian: We can consider more generic bulk SETs and boundary anomalous
SETs as in Sec. IX A 4, Remark 4-a bulk SET with global symmetry $Q$ and gauge symmetry $N$ via $1 \rightarrow N \stackrel{\text { a }}{\rightarrow} G \rightarrow Q \rightarrow 1$, and a boundary anomalous SET with global symmetry $Q$ and gauge symmetry $K \times N$ via $1 \rightarrow K \times N \rightarrow H \rightarrow Q \rightarrow 1$, where $(H / K \times N)=(G / N) \equiv Q$. This also implies $1 \rightarrow K \rightarrow$ $H \stackrel{r}{\rightarrow} G \rightarrow 1$. The generic wave function is

$$
\begin{aligned}
\Phi\left(\left\{g_{i}, n_{i_{a} i_{b}}, h_{j}, k_{j_{a} j_{b}}\right\}\right) & \underset{\longrightarrow}{\operatorname{LT}} \Phi_{R_{\mathrm{I}}}\left(\left\{g_{i}\right\},\left\{n_{i_{a} i_{b}}\right\},\left\{h_{j}^{\partial}\right\}\right) \Phi_{\partial R}\left(\left\{h_{j}^{\partial}\right\},\left\{n_{j_{a} j_{b}}\right\},\left\{k_{j_{a} j_{b}}\right\}\right), \\
& \text { where } \\
\Phi_{R_{\mathrm{I}}}\left(\left\{g_{i}\right\},\left\{n_{i_{a} i_{b}}\right\},\left\{h_{j}^{\partial}\right\}\right) \equiv & \left(\prod_{\{\ldots\}} \mathcal{V}_{d}^{G, N s_{\ldots i_{*}}}\left(\left\{g_{i}\right\}_{R_{\mathrm{I}}},\left\{r\left(h_{j}^{\partial}\right)\right\}_{\partial R}, r\left(h^{*}\right) ;\left\{n_{i_{a} i_{b}}\right\}_{R_{\mathrm{I}}, \partial R}\right)\right), \\
\Phi_{\partial R}\left(\left\{h_{j}^{\partial}\right\},\left\{n_{j_{a} j_{b}}\right\},\left\{k_{j_{a} j_{b}}\right\}\right) \equiv & \left(\prod_{\{\ldots\}} \mu_{d-1}^{H, N, K s_{i_{*}}}\left(\left\{h_{j_{a}}^{\partial}\right\}_{\partial R}, r\left(h^{*}\right) ;\left\{n_{j_{a} j_{b}} k_{j_{a} j_{b}}\right\}_{\partial R}\right)\right) .
\end{aligned}
$$




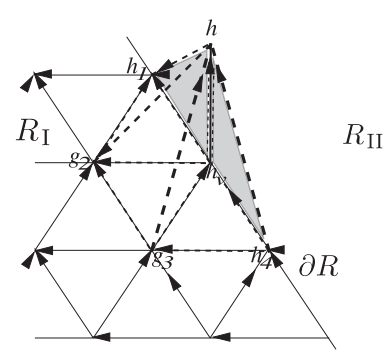

(a)

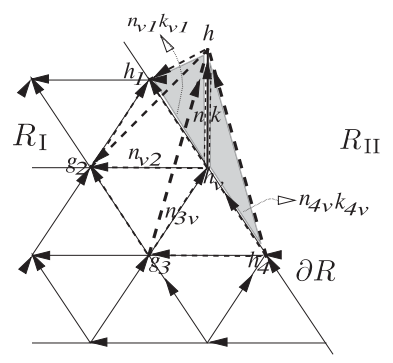

(b)
FIG. 23. (a) We consider a $G$-SPT on the spatial region $R_{\mathrm{I}}$ with a lattice. We set a trivial vacuum on the spatial region $R_{\mathrm{II}}$ and the gapped boundary of $H$-anomalous SPT on the boundary $\partial R$. The Hamiltonian $\hat{A}_{v}^{h}$ acts on the state $\left|h_{v}, h_{1}, g_{2}, g_{3}, h_{4}\right\rangle$ and is given in Eq. (9.45), which sends it to a new state $\left|h, h_{1}, g_{2}, g_{3}, h_{4}\right\rangle$ with a $U(1)$ phase. (b) Now consider a $G$-SET on the spatial region $R_{\mathrm{I}}$ lattice with a gapped boundary anomalous SET; the Hamiltonian $\hat{A}_{v}^{h, n, k}$ is given in Eq. (9.49).

Its Hamiltonian has the same form in the bulk region $R$ as $\hat{H}=-\sum_{v} \hat{A}_{v}-\sum_{f} \hat{B}_{f}$ in Eq. (9.21). But, we need to modify the boundary term on $\partial R$ to

$$
\hat{A}_{v}=\frac{1}{|H||N||K|} \sum_{h \in H, n \in N, k \in K} \hat{A}_{v}^{h, n, k} .
$$

$$
\begin{aligned}
\hat{A}_{v}^{h, n, k} \mid & \left.h_{v},\left\{h_{j}^{\partial}\right\},\left\{g_{i}\right\} ;\left\{n_{i_{a} i_{b}}\right\},\left\{k_{j_{a} j_{b}}\right\}\right\rangle \\
= & \prod_{\{\ldots\}} \mathcal{V}_{d}^{G, N s \ldots[r}\left[r(h), r\left(h_{v}\right),\left\{r\left(h_{j}^{\partial}\right)\right\},\left\{g_{i}\right\} ; n,\left\{n_{i_{a} i_{b}}\right\}\right] \\
& \times \prod_{\{\ldots\}} \mu_{d-1}^{H, N, K s \ldots}\left(h, h_{v},\left\{h_{j}^{\partial}\right\} ;\left\{n_{j_{a j} j_{b}}\right\},\left\{k_{j_{a} j_{b}}\right\}\right) \mid h \\
& \left.\times\left\{h_{j}^{\partial}\right\},\left\{g_{i}\right\} ;\left\{n_{i_{a} i_{b}}^{\prime}\right\},\left\{k_{j_{a} j_{b}}^{\prime}\right\}\right\rangle .
\end{aligned}
$$

Here, $n_{i_{a} i_{b}}^{\prime}$ and $k_{j_{a} j_{b}}^{\prime}$ are some modified link variables that may have $n$ and $k$ variables inserted.

The $\hat{B}_{f}$ term imposes trivial holonomies for the contractible loops; here, $\hat{B}_{f}$ plays an important role to constrain ground states of SETs. The bulk $\hat{B}_{f}$ imposes trivial $G$ - and $N$-holonomies for the contractible loops. The boundary $\hat{B}_{f}$ imposes trivial $H$-, $N$-, and $K$-holonomies for the contractible loops. Similar to Eq. (9.25), the bulk $\hat{B}_{f}$ constrains that $\left(\delta_{n_{v 2} n_{21} n_{1 v}=1}\right)$, and the boundary $\hat{B}_{f}$ constrains that $\left(\delta_{n_{v 2} n_{21} n_{1 v}=1}\right)\left(\delta_{k_{v 2} k_{21} k_{1 v}=1}\right)$ on each state vector associated to a two-simplex triangle.

For example, more specifically, an effective $2+1 \mathrm{D}$ Hamiltonian term $\hat{A}_{v}^{h, n, k}$ along the $1+1 \mathrm{D}$ anomalous SET gapped boundary $\partial R$, shown in Fig. 23, is written as

$$
\begin{aligned}
\hat{A}_{v}^{h, n, k}\left|h_{v}, h_{1}, g_{2}, g_{3}, h_{4} ; n_{v 1} k_{v 1}, n_{v 2}, n_{v 3}, n_{4 v} k_{4 v}\right\rangle \\
=\frac{\mu_{2}^{H}\left(h_{v}, n k h, n n_{v 1} k k_{v 1} h_{1}\right) \mu_{2}^{H}\left(h_{4}, n_{4 v} k_{4 v} h_{v}, n_{4 v} n k_{4 v} k h\right)}{\nu_{3}^{G}\left[r\left(h_{v}\right), \mathrm{a}(n) r(h), \mathrm{a}\left(n_{v 2}\right) g_{2}, \mathrm{a}\left(n_{v 1}\right) r\left(h_{1}\right)\right] \nu_{3}^{G}\left[g_{3}, \mathrm{a}\left(n_{3 v}\right) r\left(h_{v}\right), \mathrm{a}\left(n_{3 v} n\right) r(h), \mathrm{a}\left(n_{3 v} n_{v 2}\right) g_{2}\right]} \\
\quad \times \frac{1}{\nu_{3}^{G}\left[r\left(h_{4}\right), \mathrm{a}\left(n_{4 v} n_{3 v}^{-1}\right) g_{3}, \mathrm{a}\left(n_{4 v}\right) r\left(h_{v}\right), \mathrm{a}\left(n_{4 v} n\right) r(h)\right]}\left|h, h_{1}, g_{2}, g_{3}, h_{4} ; n_{v 1} n^{-1} k_{v 1} k^{-1}, n^{-1} n_{v 2}, n_{v 3} n, n_{4 v} n k_{4 v} k\right\rangle .
\end{aligned}
$$

Here, $r(h) \in G$ and $r\left(h_{i_{a}}\right) \in G$ are aimed at emphasizing that they are obtained via the epimorphism $H \stackrel{r}{\rightarrow} G$. The $\mathrm{a}(n) \in G$ and $\mathrm{a}\left(n_{i_{a} i_{b}}\right) \in G$ are aimed at emphasizing that they are obtained via the monomorphism $N \stackrel{\text { a }}{\rightarrow} G$. Since $N$ is a normal subgroup inside $G$, previously we have been abbreviating $\mathrm{a}(n)=n \in G$ for $\forall n \in N$.

In the next section, we analyze the symmetry-preserving property of such a gapped boundary system.

\section{Proof of the symmetry-preserving wave function with gapped boundary or interface}

Following the setup in Sec. IX B 3, here, we rigorously prove the wave function in Eq. (9.40) of a bulk $G$-SPT on an open manifold, while the gapped boundary has an anomalous $H$-SPT via a group extension $H / K=G$ (in Sec. IX A 4). See Fig. 24 for a geometric illustration for the proof.
We would like to interpret that the spatial bulk has two sectors $R_{\mathrm{I}} \equiv R_{\mathrm{I}}^{d}$ and $R_{\mathrm{II}} \equiv R_{\mathrm{II}}^{d}$, while the whole closed space is $R_{\mathrm{I}}^{d} \cup R_{\mathrm{II}}^{d}=M^{d}$. The SPT of symmetry group $G$ is on the $R_{\mathrm{I}}$ side, a trivial vacuum is on the $R_{\mathrm{II}}$ side, while the gapped interface $(\equiv \partial R$ ) between the two phases is symmetry enhanced to $H$. This gapped $H$ interface can be viewed as a gapped boundary for the bulk $G$-SPTs. Under the construction $1 \rightarrow K \rightarrow$ $H \stackrel{r}{\rightarrow} G \rightarrow 1$ of cocycle splitting, below, we can have an exact global $H$-symmetry transformation acting along the gapped interface, together with an exact global $G$-symmetry transformation acting on the gapped left region $R_{\mathrm{I}}$, and no symmetry transformation on the trivial right region $R_{\mathrm{II}}$. We consider the following setup:

(1) We assign a Hilbert space dimension $|H|$ on each site along the interface $\partial R$ between the $R_{\mathrm{I}}$ and the $R_{\mathrm{II}}$ regions, while the $R_{\mathrm{I}}$ region of the SPTs has a Hilbert space dimension $|G|$ on each site. 


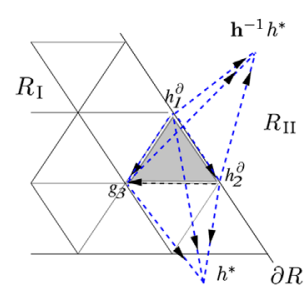

(a)

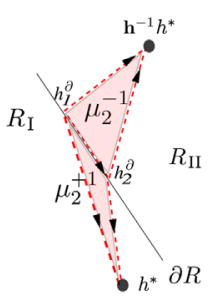

(d)

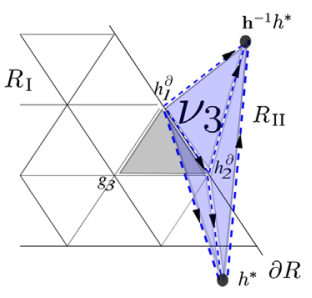

(b)

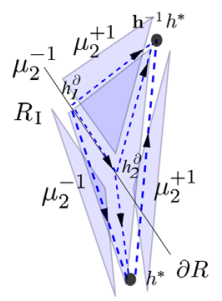

(c)

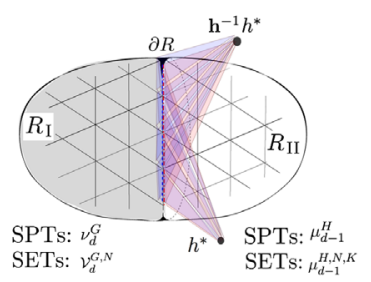

(e)
FIG. 24. We show geometry pictures how to understand the symmetry-transformation phase cancellation for the overall symmetry invariance in $2+1 \mathrm{D} / 1+1 \mathrm{D}$, which can be easily generalized to any higher-dimensional spacetime. Panel (a) shows how two pieces of $\nu_{3}$ in Eq. (9.55) contribute to the left-region wave function $\Phi_{R_{\mathrm{I}}}$ and then convert to the splitting of a $\nu_{3}$ into four pieces of two-cochains in panels (b) and (c) as in Eq. (9.57). Panel (d) shows how two pieces of $\mu_{2}$ in Eq. (9.59) contribute to the interface-region wave function $\Phi_{\partial R}$. Panel (e) shows how, on a closed interface $\partial R$ (here, an $S^{1}$ ), the symmetry transformation on the combined wave function $\Phi_{R_{\mathrm{I}}} \cdot \Phi_{\partial R}$ canceling with each other to 1 as the symmetry invariance achieved in Eq. (9.60).

(2) We require the dimension of Hilbert space on the additional site $i_{*}$ assigned with $h^{*}$ outside $M^{d}$ has a Hilbert space dimension $|H|$. We also have an additional virtual site $i_{*}^{\prime}$ assigned with $\mathbf{h}^{-1} h^{*}$ for $\forall \mathbf{h} \in H$, such that $r(\mathbf{h}) \equiv g, r\left(h^{*}\right) \equiv g^{*}$, and

$$
r\left(\mathbf{h}^{-1} h^{*}\right)=r\left(\mathbf{h}^{-1}\right) r\left(h^{*}\right)=\mathbf{g}^{-1} g^{*}
$$

We also set that the site $i_{*}{ }^{\prime}$ has a Hilbert space dimension $|H|$. The condition (2) is important in order to split the cocycle on the $R_{\mathrm{I}}$ region that touches the interface.

(3) We consider the algebraic-structure-preserving map from $H$ to $G$ with $r(h)=g$, the same map of $H \stackrel{r}{\rightarrow} G$. The symmetry transformation sends $\left|g_{j}\right\rangle \rightarrow\left|r(\mathbf{h}) g_{j}\right\rangle=$ $\left|\mathbf{g} g_{j}\right\rangle$ when the dimension of Hilbert space is $|G|$ on the site $j$. The symmetry transformation sends $\left|h_{j}\right\rangle \rightarrow\left|\mathbf{h} h_{j}\right\rangle$ when the Hilbert space dimension is $|H|$ on the site $j$.

The exact global $G$-symmetry transformation on the left region $R_{I}$ and the exact global $H$-symmetry transformation along the interface yield global $U(1)$ phases to the wave function, and the global $U(1)$ phases need to cancel out to 1 . The cancellation of global $U(1)$ phases of $G$-symmetry and $H$-symmetry transformations may be viewed as anomaly free for the whole bulk and the interface. The wave function is only symmetry invariant if we consider the whole system together.

Now, consider the group manifold that has the left $\left(R_{\mathrm{I}}\right)$ sector of group $G$ and the right sector of a trivial vacuum, and all sectors can be lifted to the larger group $H$. Again, we set that $g_{\mathrm{I}}^{*}=g_{\mathrm{II}}^{*}=g^{*}=r_{V}\left(h^{*}\right)=h^{*}=1$. In general, we can easily generalize our result to any dimension. Without losing generality, let us take a specific example in $2+1 \mathrm{D}$, and let us consider the two-dimensional space lattice defined on a two-sphere $S^{2}$. The $S^{2}$ can be regarded as two two-disks $D^{2}$ glued together along the $S^{1}$ boundary. Let us call the two $D^{2}$ disks $D_{R_{\mathrm{I}}}^{2}$ assigned with $G_{\mathrm{I}}$ on each site and $D_{R_{\mathrm{II}}}^{2}$ assigned with $G_{\mathrm{II}}$ on each site. Along the $S^{1}$ boundary, we assign $H$ on each site. The wave function on the whole $S^{2}$ surface is evolved from an additional point $i^{*}$ assigned $g^{*}=r\left(h^{*}\right)$. Thus, the wave function can be determined by assigning the three-cocycle into this spacetime volume of the $D^{3}$ ball (whose center is $i^{*}$ and whose spatial sector is $S^{2}$ ).

For SPTs, we use the homogeneous cocycle denoted $\nu_{d}^{G_{s}}$ and cochain $\mu_{d-1}^{H}$, and we follow the wave function $\Phi\left(\left\{g_{i}, h_{j}^{\partial}\right\}\right) \equiv \Phi_{R_{\mathrm{I}}}\left(\left\{g_{i}\right\},\left\{h_{j}^{\partial}\right\}\right) \Phi_{\partial R}\left(\left\{h_{j}^{\partial}\right\}\right)$ in Eq. (9.42). Here, we arrange the wave function separated into a few parts:

$$
\begin{aligned}
\Phi_{R_{\mathrm{I}}}\left(\left\{g_{i}\right\},\left\{h_{j}^{\partial}\right\}\right) & \equiv \prod_{\{\ldots\}} \nu_{3}^{G s}\left[g_{i_{1}}, g_{i_{2}}, g_{i_{3}}, r\left(h^{*}\right)\right] \nu_{3}^{G s}\left[r\left(h_{j_{1}}^{\partial}\right), g_{i_{2}}, g_{i_{3}}, r\left(h^{*}\right)\right] \nu_{3}^{G s}\left[r\left(h_{j_{1}}^{\partial}\right), r\left(h_{j_{2}}^{\partial}\right), g_{i_{3}}, r\left(h^{*}\right)\right] \\
\Phi_{\partial R}\left(\left\{h_{j}^{\partial}\right\}\right) & \equiv \prod_{j} \mu_{2}^{H s}\left(h_{j}^{\partial}, h_{j+1}^{\partial}, h^{*}\right)
\end{aligned}
$$

Again, there are orientations $s= \pm 1$ for each term.

Below, we verify that the wave function $\Phi\left(\left\{g_{i}, h_{j}^{\partial}\right\}\right)$ is invariant under the global-symmetry transformation $\hat{\mathbf{S}}_{\mathrm{sym}}$. It means that we can show that $\Phi\left(\left\{g_{i}, h_{j}^{\partial}\right\}\right)$ is equal to

$$
\hat{\mathbf{S}}_{\mathrm{sym}} \Phi\left(\left\{g_{i}, h_{j}^{\partial}\right\}\right)=\Phi\left[\left\{\left(r(\mathbf{h}) \cdot g_{i}\right),\left(\mathbf{h} \cdot h_{j}^{\partial}\right)\right\}\right]
$$

We also denote the change $r(\mathbf{h}) \equiv \mathbf{g}$ in $G$. The above shows the symmetry transformation acts on the wave function. Conversely, we can consider the equivalent dual picture that the symmetry transformation acts on the state vector in the Hilbert space. Either way leads to the same conclusion. Since 
$\hat{\mathbf{S}}_{\mathrm{sym}} \Phi\left(\left\{g_{i}, h_{j}^{\partial}\right\}\right)=\left[\frac{\Phi\left[\left\{\left(r(\mathbf{h}) \cdot g_{i}\right),\left(\mathbf{h} \cdot h_{j}^{\partial}\right)\right\}\right]}{\Phi\left(\left\{g_{i}, h_{j}^{\partial}\right\}\right)}\right] \Phi\left(\left\{g_{i}, h_{j}^{\partial}\right\}\right)$,

we need to show that the factor in the bracket $[\ldots]$ is 1 to prove the global symmetry preservation. The $G$-symmetry on the region $R_{\mathrm{I}}$ must be able to be lifted to some $H$-symmetry on the whole regions $R_{\mathrm{I}}$ including the interface $\partial R$, based on the fact that $H \stackrel{r}{\rightarrow} G$ is surjective. We remind the readers that $\mathbf{g} \equiv r(\mathbf{h})$, $g^{*} \equiv r\left(h^{*}\right)$. Namely, it is effectively the $H$-symmetry transformation on the whole system.

In region $R_{\mathrm{I}}$, the wave function change $\left[\nu_{3}\left(g \cdot g_{1}\right.\right.$, $\left.\left.g \cdot g_{2}, g \cdot g_{3}, g^{*}\right) / \nu_{3}\left(g_{1}, g_{2}, g_{3}, g^{*}\right)\right]=\left[\nu_{3}\left(g_{1}, g_{2}, g_{3}, g^{-1} \cdot g^{*}\right) /\right.$ $\left.\nu_{3}\left(g_{1}, g_{2}, g_{3}, g^{*}\right)\right]$ can be simplified further based on a $d$-cocycle condition,

$$
\begin{gathered}
\left(\delta \nu_{3}\right)\left(g_{i}, g_{j}, g^{*}, \mathbf{g}^{-1} \cdot g^{*}\right)=1 \\
\Rightarrow \frac{\nu_{3}\left(g_{1}, g_{2}, g_{3}, g^{-1} \cdot g^{*}\right)}{\nu_{3}\left(g_{1}, g_{2}, g_{3}, g^{*}\right)} \\
=\frac{\nu_{3}\left(g_{2}, g_{3}, g^{*}, \mathbf{g}^{-1} \cdot g^{*}\right) \nu_{3}\left(g_{1}, g_{2}, g^{*}, \mathbf{g}^{-1} \cdot g^{*}\right)}{\nu_{3}\left(g_{1}, g_{3}, g^{*}, \mathbf{g}^{-1} \cdot g^{*}\right)} .
\end{gathered}
$$

Here, for convenience, let us denote $\overline{g_{i} g_{j}}$ as a link connecting two vertices $i$ and $j$, where two vertices are assigned with $g_{i}$ and $g_{j}$, respectively. Notice that the three-cocycle $\nu_{3}\left(g_{i}, g_{j}, g^{*}, \mathbf{g}^{-1} \cdot g^{*}\right)$, which contains a link $\overline{g_{i} g_{j}}$, is canceled out, because there exists a neighbor term that shares the same link $\overline{g_{i} g_{j}}$ and that contributes the same factor with opposite orientation, and thus, opposite sign for $s= \pm 1$. The only subtle type of terms that survive and that require further analysis is $\nu_{3}\left[r\left(h_{i}^{\partial}\right), r\left(h_{j}^{\partial}\right), g^{*}, \mathbf{g}^{-1} \cdot g^{*}\right]$, which contains a link with two vertices $\overline{h_{i}^{\partial} h_{j}^{\partial}}$ on the interface $\partial R$. If we approach from the region $R_{\mathrm{I}}$, we see that

$$
\begin{aligned}
& \frac{\nu_{3}\left(r\left(h_{1}^{\partial}\right), r\left(h_{2}^{\partial}\right), g_{3}, \mathbf{g}^{-1} \cdot g^{*}\right)}{\nu_{3}\left(r\left(h_{1}^{\partial}\right), r\left(h_{2}^{\partial}\right), g_{3}, g^{*}\right)} \\
& =\frac{\nu_{3}\left(r\left(h_{2}^{\partial}\right), g_{3}, g^{*}, \mathbf{g}^{-1} \cdot g^{*}\right) \nu_{3}\left(r\left(h_{1}^{\partial}\right), r\left(h_{2}^{\partial}\right), g^{*}, \mathbf{g}^{-1} \cdot g^{*}\right)}{\nu_{3}\left(r\left(h_{1}^{\partial}\right), g_{3}, g^{*}, \mathbf{g}^{-1} \cdot g^{*}\right)} .
\end{aligned}
$$

All the terms on the right-hand side cancel with some other terms in the product $\prod_{\{\ldots\}}$, which share the same links connecting $\overline{h_{1}^{\partial} g_{3}}$ and $\overline{h_{2}^{\partial} g_{3}}$ on the same region $R_{I}$, except for the $\nu_{3}\left[r\left(h_{1}^{\partial}\right), r\left(h_{2}^{\partial}\right), g^{*}, \mathbf{g}^{-1} \cdot g^{*}\right]$ term that touches the link $\overline{h_{1}^{\partial} h_{2}^{\partial}}$. We would like to split the three-cocycle $\nu_{3}^{G}$ that touches the link $\overline{h_{i}^{\partial} h_{j}^{\partial}}$ into two-cochains $\mu_{2}^{H}$ :

$$
\begin{aligned}
\nu_{3}^{G} & {\left[r\left(h_{1}^{\partial}\right), r\left(h_{2}^{\partial}\right), r\left(h^{*}\right), r\left(\mathbf{h}^{-1} \cdot h^{*}\right)\right] } \\
& =\nu_{3}^{H}\left(h_{1}^{\partial}, h_{2}^{\partial}, h^{*}, \mathbf{h}^{-1} \cdot h^{*}\right) \\
& =\left(\delta \mu_{2}^{H}\right)\left(h_{1}^{\partial}, h_{2}^{\partial}, h^{*}, \mathbf{h}^{-1} \cdot h^{*}\right) \\
& =\frac{\mu_{2}^{H}\left(h_{2}^{\partial}, h^{*}, \mathbf{h}^{-1} \cdot h^{*}\right) \mu_{2}^{H}\left(h_{1}^{\partial}, h_{2}^{\partial}, \mathbf{h}^{-1} \cdot h^{*}\right)}{\mu_{2}^{H}\left(h_{1}^{\partial}, h^{*}, \mathbf{h}^{-1} \cdot h^{*}\right) \mu_{2}^{H}\left(h_{1}^{\partial}, h_{2}^{\partial}, h^{*}\right)} .
\end{aligned}
$$

We shall consider all such splitting terms along the interface. As an example, for the $1+1 \mathrm{D}$ interface on a spatial ring with a total number of $N$ sites and $N$ links $\overline{\left(h_{j}^{\partial} h_{j+1}^{\partial}\right)}$, where $i=1, \ldots, N(\bmod N)$, we obtain

$$
\begin{aligned}
& \prod_{j=1}^{\mathrm{N}} \nu_{3}^{G}\left[r\left(h_{j}^{\partial}\right), r\left(h_{j+1}^{\partial}\right), r\left(h^{*}\right), r\left(\mathbf{h}^{-1} \cdot h^{*}\right)\right] \\
& \quad=\prod_{j=1}^{\mathrm{N}} \frac{\mu_{2}^{H}\left(h_{j+1}^{\partial}, h^{*}, \mathbf{h}^{-1} \cdot h^{*}\right)}{\mu_{2}^{H}\left(h_{j}^{\partial}, h^{*}, \mathbf{h}^{-1} \cdot h^{*}\right)} \prod_{j=1}^{\mathrm{N}} \frac{\mu_{2}^{H}\left(h_{j}^{\partial}, h_{j+1}^{\partial}, \mathbf{h}^{-1} \cdot h^{*}\right)}{\mu_{2}^{H}\left(h_{j}^{\partial}, h_{j+1}^{\partial}, h^{*}\right)} \\
& \quad=\prod_{j=1}^{\mathrm{N}} \frac{\mu_{2}^{H}\left(h_{j}^{\partial}, h_{j+1}^{\partial}, \mathbf{h}^{-1} \cdot h^{*}\right)}{\mu_{2}^{H}\left(h_{j}^{\partial}, h_{j+1}^{\partial}, h^{*}\right)} .
\end{aligned}
$$

The first is based on Eq. (9.56) on a ring. For the second equality, we use the fact that $\prod_{j=1}^{\mathrm{N}}\left[\mu_{2}^{H}\left(h_{j+1}^{\partial}, h^{*}, \mathbf{h}^{-1} \cdot h^{*}\right) /\right.$ $\left.\mu_{2}^{H}\left(h_{j}^{\partial}, h^{*}, \mathbf{h}^{-1} \cdot h^{*}\right)\right]=1$ cancels out on a closed ring. Combined with the fact that a homogeneous cochain does not change under symmetry transformation if inputs do not contain $h^{*}$, because the homogenous cocycle satisfies $\left[\mu_{2}^{H}\left(\mathbf{h} \cdot h_{i}, \mathbf{h} \cdot h_{j}, \mathbf{h} \cdot h_{k}\right) / \mu_{2}^{H}\left(h_{i}, h_{j}, h_{k}\right)\right]=1$, so far we derive that

$$
\frac{\Phi_{R_{\mathrm{I}}}\left(\left\{r(\mathbf{h}) \cdot g_{i}\right\},\left\{\mathbf{h} \cdot h_{j}^{\partial}\right\}\right)}{\Phi_{R_{\mathrm{I}}}\left(\left\{g_{i}\right\},\left\{h_{j}^{\partial}\right\}\right)}=\prod_{j=1}^{\mathrm{N}} \frac{\mu_{2}^{H}\left(h_{j}^{\partial}, h_{j+1}^{\partial}, \mathbf{h}^{-1} \cdot h^{*}\right)}{\mu_{2}^{H}\left(h_{j}^{\partial}, h_{j+1}^{\partial}, h^{*}\right)} .
$$

We can also see that the remaining part of the wave function is $\Phi_{\partial R}\left(\left\{h_{j}^{\partial}\right\}\right)=\prod_{j=1}^{\mathrm{N}} \mu_{2}^{H}\left(h_{j}^{\partial}, h_{j+1}^{\partial}, h^{*}\right)^{-1}$, where the inverse with $s=-1$ is due to the opposite orientation accounted for from the other side $R_{\mathrm{II}}$. Its symmetry transformation becomes

$$
\frac{\Phi_{\partial R}\left(\left\{\mathbf{h} \cdot h_{j}^{\partial}\right\}\right)}{\Phi_{\partial R}\left(\left\{h_{j}^{\partial}\right\}\right)} \equiv \prod_{j=1}^{\mathrm{N}}\left(\frac{\mu_{2}^{H}\left(h_{j}^{\partial}, h_{j+1}^{\partial}, \mathbf{h}^{-1} \cdot h^{*}\right)}{\mu_{2}^{H}\left(h_{j}^{\partial}, h_{j+1}^{\partial}, h^{*}\right)}\right)^{-1} .
$$

Thus, the phases in Eqs. (9.58) and (9.59) cancel perfectly, and the whole wave function $\Phi\left(\left\{g_{i}, h_{j}^{\partial}\right\}\right) \equiv \Phi_{R_{\mathrm{I}}}\left(\left\{g_{i}\right\}\right.$, $\left.\left\{h_{j}^{\partial}\right\}\right) \Phi_{\partial R}\left(\left\{h_{j}^{\partial}\right\}\right)$ is invariant under the symmetry transformation: 


$$
\begin{aligned}
\hat{\mathbf{S}}_{\mathrm{sym}} \Phi\left(\left\{g_{i}, h_{j}^{\partial}\right\}\right)= & \frac{\Phi_{R_{\mathrm{I}}}\left(\left\{r(\mathbf{h}) \cdot g_{i}\right\},\left\{\mathbf{h} \cdot h_{j}^{\partial}\right\}\right)}{\Phi_{R_{\mathrm{I}}}\left(\left\{g_{i}\right\},\left\{h_{j}^{\partial}\right\}\right)} \frac{\Phi_{\partial R}\left(\left\{\mathbf{h} \cdot h_{j}^{\partial}\right\}\right)}{\Phi_{\partial R}\left(\left\{h_{j}^{\partial}\right\}\right)} \\
& \cdot \Phi\left(\left\{g_{i}, h_{j}^{\partial}\right\}\right)=1 \cdot \Phi\left(\left\{g_{i}, h_{j}^{\partial}\right\}\right) .
\end{aligned}
$$

In Fig. 24, we show a neat geometrical way to understand the symmetry-transformation phase cancellation for the symmetry invariance. For any higher $d$-dimensional spacetime, we can give the same proof by replacing $\mu_{2}^{H}$ in Eqs. (9.58) and (9.59) with $\mu_{d-1}^{H}$. It is easy to confirm that our proof on a symmetry-preserving gapped interface holds for any higher-dimensional generalization (q.e.d.).

We can apply a similar proof for the global-symmetrypreserving property of the SET version of the wave function in Eq. (9.46) to show

$$
\begin{aligned}
\hat{\mathbf{S}}_{\mathrm{sym}} & \Phi\left(\left\{g_{i}, n_{i_{a} i_{b}}, h_{j}, k_{j_{a} j_{b}}\right\}\right) \\
& =\Phi\left(\left\{r(\mathbf{h}) \cdot g_{i}, n_{i_{a} i_{b}}, \mathbf{h} \cdot h_{j}, k_{j_{a} j_{b}}\right\}\right) \\
& =\Phi\left(\left\{g_{i}, n_{i_{a} i_{b}}, h_{j}, k_{j_{a} j_{b}}\right\}\right) .
\end{aligned}
$$

To prove this, we may regard that $\mathbf{h} \cdot h_{j} \equiv h_{j} \cdot \mathbf{h}^{\prime}$, where $\mathbf{h}^{\prime}=h_{j}^{-1} \mathbf{h} h_{j}$. Similarly, $r(\mathbf{h}) \cdot g_{i} \equiv \mathbf{g} \cdot g_{i} \equiv g_{i} \cdot \mathbf{g}^{\prime}$, we find that $\mathbf{g}^{\prime}=g_{j}^{-1} \mathbf{g} g_{j}=r\left(h_{j}^{-1}\right) r(\mathbf{h}) r\left(h_{j}\right)=r\left(h_{j}^{-1} \mathbf{h} h_{j}\right)=r\left(\mathbf{h}^{\prime}\right)$. Regardless of the branch structure for vertex ordering, we can convert the symmetry transformation, from acting on the left of the group elements to that acting on the right of the group elements. This trick can facilitate the proof that the SET wave function is invariant under global symmetry, even in the presence of gapped interfaces.

\section{More remarks}

Here are a summary and some more remarks:

(1) Global enhanced H-symmetry invariant: We have shown that the SPT wave function on a whole system is invariant under $G$-symmetry transformation in the bulk $R_{\mathrm{I}}$ together under $H$-symmetry transformation on the interface $\partial R$. The symmetry transformation is fixed by $H \stackrel{r}{\rightarrow} G$, and we may view that the symmetry is enhanced to $H$ for the whole system.

(2) Global K-symmetry on the boundary or interface: Under the construction $1 \rightarrow K \rightarrow H \stackrel{r}{\rightarrow} G \rightarrow 1$ for $G$-bulk SPTs and anomalous boundary $H$-SPTs, the $K$ is trivial in the bulk as $r(k)=1 \in G$ for $k \in K$. How about $K$-symmetry transformation on the interface? It is easy to check there is no local $K$-symmetry on the interface, since $\Phi_{\partial R}\left(\left\{\mathbf{k}_{j} \cdot h_{j}^{\partial}\right\}\right) \neq \Phi_{\partial R}\left(\left\{h_{j}^{\partial}\right\}\right)$ for arbitrary local $\mathbf{k}_{j} \in K$ transformation on each site $j$. However, below we can prove that there is a global $K$-symmetry applying on the boundary or interface, namely,

$$
\Phi_{\partial R}\left(\left\{\mathbf{k} \cdot h_{j}^{\partial}\right\}\right)=\Phi_{\partial R}\left(\left\{h_{j}^{\partial}\right\}\right) .
$$

Proof: Without losing generality, consider the 1+1D boundary of $2+1 \mathrm{D}$ SPTs. We see that

$\Phi_{\partial R}\left(\left\{\mathbf{k} \cdot h_{j}^{\partial}\right\}\right)=\prod_{j=1}^{\mathrm{N}} \frac{\mu_{2}^{H}\left(\mathbf{k} h_{j}^{\partial}, \mathbf{k} h_{j+1}^{\partial}, h^{*}\right)}{\mu_{2}^{H}\left(h_{j}^{\partial}, h_{j+1}^{\partial}, h^{*}\right)} \cdot \Phi_{\partial R}\left(\left\{h_{j}^{\partial}\right\}\right)=\prod_{j=1}^{\mathrm{N}} \frac{\mu_{2}^{H}\left(h_{j}^{\partial}, h_{j+1}^{\partial}, \mathbf{k}^{-1} h^{*}\right)}{\mu_{2}^{H}\left(h_{j}^{\partial}, h_{j+1}^{\partial}, h^{*}\right)} \cdot \Phi_{\partial R}\left(\left\{h_{j}^{\partial}\right\}\right)=\Phi_{\partial R}\left(\left\{h_{j}^{\partial}\right\}\right)$,

where in the last equality we use the fact of three-cocycle splitting and $r(\mathbf{k})=1 \in G$, so

$$
\begin{aligned}
1 & =\nu_{3}\left(r\left(h_{j}^{\partial}\right), r\left(h_{j+1}^{\partial}\right), r\left(h^{*}\right)=g^{*}, r\left(\mathbf{k}^{-1} \cdot h^{*}\right)=g^{*}\right)=\frac{\mu_{2}\left(h_{j+1}^{\partial}, h^{*}, \mathbf{k}^{-1} \cdot h^{*}\right)}{\mu_{2}\left(h_{j}^{\partial}, h^{*}, \mathbf{k}^{-1} \cdot h^{*}\right)} \frac{\mu_{2}\left(h_{j}^{\partial}, h_{j+1}^{\partial}, \mathbf{k}^{-1} \cdot h^{*}\right)}{\mu_{2}\left(h_{j}^{\partial}, h_{j+1}^{\partial}, h^{*}\right)} \\
\Rightarrow 1 & =\prod_{j=1}^{\mathrm{N}} 1=\prod_{j=1}^{\mathrm{N}} \frac{\mu_{2}\left(h_{j+1}^{\partial}, h^{*}, \mathbf{k}^{-1} \cdot h^{*}\right)}{\mu_{2}\left(h_{j}^{\partial}, h^{*}, \mathbf{k}^{-1} \cdot h^{*}\right)} \prod_{j=1}^{\mathrm{N}} \frac{\mu_{2}\left(h_{j}^{\partial}, h_{j+1}^{\partial}, \mathbf{k}^{-1} \cdot h^{*}\right)}{\mu_{2}\left(h_{j}^{\partial}, h_{j+1}^{\partial}, h^{*}\right)} \\
\Rightarrow & 1=1 \cdot \prod_{j=1}^{\mathrm{N}} \frac{\mu_{2}\left(h_{j}^{\partial}, h_{j+1}^{\partial}, \mathbf{k}^{-1} \cdot h^{*}\right)}{\mu_{2}\left(h_{j}^{\partial}, h_{j+1}^{\partial}, h^{*}\right)} .
\end{aligned}
$$

(3) Gauging SPTs to SETs: Since there is a global $K$-symmetry on the boundary or interface, we can partially or fully gauge this $K$-symmetry. We can also gauge a normal subgroup $N$ of the global $G$-symmetry of $G$-SPTs-however, to gauge $N$ in the bulk, we also need to gauge the $N$ for the anomalous $H$-SPTs on the boundary or interface. By gauging the normal subgroups $N$ and $K$, this gives rise to SETs of Sec. IX A 4, Remark 4.
(4) Degenerate ground states and holonomies for the boundary anomalous SETs: If the gapped boundary is on a compact space with nontrivial cycles, there can be nontrivial holonomies for the gapped boundary anomalous SETs. For example, for a $2+1 \mathrm{D}$ SPT on a two-disk $D^{2}$ and its $1+1 \mathrm{D}$ anomalous SETs on a one-circle $S^{1}$, or, for a $3+1 \mathrm{D}$ SPTs on a solid torus $D^{2} \times S^{1}$ and its $2+1 \mathrm{D}$ anomalous SETs on a two-torus $T^{2}$, their nontrivial boundary 
holonomies imply the ground-state degeneracy (GSD). We will explicitly compute such GSDs for some examples in Appendix D, such as $0 \rightarrow Z_{2}^{K} \rightarrow$ $Z_{4}^{H} \rightarrow Z_{2}^{G} \rightarrow 0$ in Sec. D 4 a and $1 \rightarrow Z_{4}^{K} \rightarrow Q_{8}^{H} \rightarrow$ $Z_{2}^{G} \rightarrow 1$ in Sec. D 10 a.

(5) Gapped interfaces by folding trick: Again, based on the folding trick, we can construct a wave function and lattice Hamiltonian of gapped interfaces between two topological phases in Sec. IX A 5, and we still can prove the symmetry-preserving wave function.

\section{CONCLUSION}

Some concluding and additional remarks follow:

(1) We provide a UV complete lattice regularization of the Hamiltonian and path integral definition of gapped interfaces based on the symmetry-extension mechanism, partly rooted in Ref. [51]. Presumably, some of the other phenomena studied in Ref. [51] could also be examined based on our lattice regularized setting.

(2) The anomalous non-on-site $G$-symmetry at the boundary indicates that, if we couple the $G$-symmetric boundary to the weakly fluctuating background probed gauge field of $G$, there is an anomaly in $G$ (in the same language as in particle physics and highenergy theory) along the boundary. The $G$-anomaly can be a gauge anomaly (e.g., for an internal unitary $G$-symmetry) or a mixed gauge-gravitational anomaly (e.g., for a $G$-symmetry that contains an antiunitary time-reversal symmetry $Z_{2}^{T}$ ). The key ingredient of our approach is based on the fact that certain nonperturbative global anomalies in $G$ at the boundary become anomaly free in $H$, when $G$ is pulled back to $H$ (see Sec. IV E).

(3) Given some bulk $G$-SPT states, our formulation finds their possible $H$-symmetry-extended and $G$-symmetry-preserving gapped boundaries, via a suitable group extension $1 \rightarrow K \rightarrow H \rightarrow G \rightarrow 1$ [75]. To construct an $H$-symmetry-extended gapped boundary, we actually require a weaker condition on the group extension that $K$ may be a finite group or a continuous group, in any bulk dimension $\geq 1+1 \mathrm{D}$. To construct a $G$-symmetry-preserving topologically ordered gapped boundary, we further require a stronger condition on the group extension that $K$ is a finite group, in order to have a boundary deconfined $K$-gauge theory, for a $3+1 \mathrm{D}$ bulk and above.

(4) When $G, H$, and $K$ are finite groups, we can prove that there always exist $H$-symmetry extended gapped boundaries (in any bulk dimension $\geq 1+1 \mathrm{D})$ and there always exist $G$-symmetrypreserving gapped boundaries (for $3+1 \mathrm{D}$ bulk and above). The gauge anomaly associated to a finite symmetry group $G$ must be a nonperturbative global anomaly. The cohomology or cobordism group of a finite $G$ only contains the torsion part, which indicates the nonperturbative anomalies.

We believe that the argument remains valid, even when $G$ and $H$ are infinite continuous compact groups, but $K$ remains a finite group. In this case, the boundary dynamics still yields a deconfined $K$-gauge theory, given that the bulk dimensions are larger or equal to $3+1 \mathrm{D}$ (see Sec. VI). (When the bulk is $2+1 \mathrm{D}$, we comment in the next remark.)

When $G$ is a continuous group for the bulk $G$-SPTs, the boundary could have both perturbative anomalies (e.g., captured by a one-loop Feynman diagram) and nonperturbative global anomalies, detected by coupling the boundary to $G$-gauge fields [77]. The perturbative anomalies do not offer any symmetry-preserving surface topological orders. In contrast, some of the nonperturbative global anomalies can offer a symmetry-preserving surface topological order as long as our construction trivializes the $G$-anomaly in $H$.

(5) We apply our symmetry-preserving gapped interface construction to the $2+1 \mathrm{D}$ bulk and $1+1 \mathrm{D}$ boundary. For the $1+1 \mathrm{D}$ topologically ordered $K$-gauge theory on the boundary of a finite or continuous group symmetry of $2+1 \mathrm{D} G$-SPTs, we find an interesting phenomenon that the $1+1 \mathrm{D}$ boundary deconfined $K$-gauge theory states develop longrange orders that spontaneously break the $G$ symmetry (see Sec. IV H). The $1+1 \mathrm{D}$ boundary deconfined and confined gauge theory states belong to the same phase; namely, they are both symmetrybreaking states connected without phase transitions.

Examples include those of a finite gauge group $K$, and a global symmetry $G$ containing discrete unitary or antiunitary global symmetry sectors that can be spontaneously broken. For instance, in Sec. III C and Appendixes D $2 \mathrm{~d}$ and D22, we show that the unitary $Z_{2}^{G}$-symmetry of a $1+1 \mathrm{D} Z_{2}^{K}$ gauge theory is spontaneously broken, on the boundary of $2+1 \mathrm{D}$ $Z_{2}^{G}$-SPTs. In Appendix D 22, we also show that the antiunitary time-reversal $Z_{2}^{T}$-symmetry of a $1+1 \mathrm{D}$ $Z_{2}^{K}$ gauge theory is spontaneously broken, on the boundary of a $2+1 \mathrm{D}$ bosonic $U(1) \rtimes Z_{2}^{T}$-topological insulator and a $Z_{2} \rtimes Z_{2}^{T}$-topological superconductor. This is, so far, consistent with the fact that there is no robust intrinsic topological order in $1+1 \mathrm{D}$ robust against any local perturbations.

(6) Our approach shall be applicable to obtain gapped interfaces of more generic bosonic and fermionic topological states (other than the fermionic CZX model in Appendix B), including topological states from the beyond-symmetry-group cohomology and cobordism approach (Secs. VI and VII). It will be interesting to establish this result with more concrete examples. 
(vii) In Appendix D, we systematically construct various symmetry-extended gapped boundaries for topological states in various dimensions (choosing homogeneous cocycles for SPTs and inhomogeneous cocycles for topological orders). We can also combine results in different subsections in Appendix D and use the folding trick to obtain the gapped interfaces between topological states.

The previously known gapped interfaces for the $Z_{2}$ toric code and $Z_{2}$ double-semion model can be achieved by certain (gauge-)symmetrybreaking sine-Gordon cosine interactions at strong couplings. The previously known gapped interfaces of $2+1 \mathrm{D}$ twisted quantum double models $D^{\omega_{3}}(G)$ and Dijkgraaf-Witten gauge theories can also be obtained through such a (gauge-)symmetry-breaking mechanism or anyon condensation [78-84]; see Appendix F. It is known that there are two types of gapped boundaries for the $Z_{2}$ toric code, one type of gapped boundary for the $Z_{2}$ double-semion model, and two types of gapped interfaces between the $Z_{2}$ toric code and $Z_{2}$ double-semion model [84]. More generally, we systematically show gauge-symmetry-breaking gapped interfaces in any dimension, in Appendix F, including $2+1 \mathrm{D}$ (ours reproduce the results in the previous literature) and the less-studied $3+1 \mathrm{D}$.

However, we can construct other new types of gapped interfaces between $Z_{2}$ toric code and $Z_{2}$ double-semion models via a symmetryextension mechanism, such as the examples given in Appendices D 4's 2+1/1+1D under $0 \rightarrow Z_{2}^{K} \rightarrow$ $Z_{4}^{H} \rightarrow Z_{2}^{G} \rightarrow 0$, Appendix D 10's $2+1 / 1+1 \mathrm{D}$ under $1 \rightarrow Z_{4}^{K} \rightarrow Q_{8}^{H} \rightarrow Z_{2}^{G} \rightarrow 1$, and more. Our new gapped interface has an enhanced Hilbert space and to a certain degree an enhanced gauge symmetry; the first new type of gapped interface has $H=Z_{4}$, and the second new type of gapped interface has $H=Q_{8}$. Through a symmetry-extension mechanism, we can construct new types of gapped boundaries or interfaces in $2+1 \mathrm{D}, 3+1 \mathrm{D}$, and any higher dimensions [85].

More generally, our framework encompasses the mixed symmetry breaking, symmetry extension, and dynamically gauging mechanisms to generate gapped interfaces.

(8) Future application: Gapped interfaces via gaugesymmetry breaking or anyon condensations have recently found their applications in topological quantum computation (see Ref. [87] and references therein for $2+1 \mathrm{D}$ bulk systems). We hope that our new types of gapped interfaces via global or gauge symmetry extensions in any dimension have analogous potential applications, for science and technology, in the future.

\section{ACKNOWLEDGMENTS}

J. W. thanks Thomas Church, Tian Lan, Kantaro Ohmori, and Pavel Putrov for conversations. J. W. gratefully acknowledges the Corning Glass Works Foundation Fellowship and the NSF. X.-G. W.'s research is supported by NSF Grants No. DMR-1506475 and No. NSFC 11274192. J. W.'s work was performed in part at the Aspen Center for Physics, which is supported by National Science Foundation Grant No. PHY-1066293. The research at I. A. S. is supported by the NSF Grants No. PHY-1606531 and No. PHY-1314311.

\section{APPENDIX A: LOW-ENERGY EFFECTIVE THEORY FOR THE BOUNDARIES OF THE CZX MODEL}

\section{Low-energy effective theory for the second boundary of the CZX model: A $1+1 D$ model with an on-site $Z_{4}^{H}$-symmetry}

In Sec. III B, we described a gapped boundary state of the CZX model in which the $Z_{2}^{G}$ bulk symmetry is extended to a $Z_{4}^{H}$-symmetry along the boundary. The model as described there is gapped in both bulk and boundary, and there is no hierarchy of energy scales: The energy gaps in bulk and along the boundary are comparable.

This is a physically sensible state of affairs in condensed matter physics, but nonetheless one might ask what sort of model would have such a hierarchy of scales. In this section, we will describe several possibilities. As a result, we obtain several pure $1+1 \mathrm{D}$ models as the effective boundary theories for the CZX model.

One approach is simply to reduce the coefficient of the boundary plaquette term $H_{p}^{\text {bdry }}$ in the Hamiltonian. In this limit (see Fig. 4), the low-energy degrees of freedom at the boundary are described by three spins per unit cell: $\boldsymbol{\sigma}_{i_{-}}, \boldsymbol{\sigma}_{i_{+}}$, and a composite spin described by the two spins on the black dots next to $\sigma_{i_{-}}$and $\boldsymbol{\sigma}_{i_{+}}$, which are locked due to the projector $P_{p}^{r}$ from the neighboring Hamiltonian.

Here, we would like to reduce the boundary degrees of freedom further. To do so, we will consider a slightly different boundary, by omitting the $H_{p}^{\text {bdry }}$ terms in the Hamiltonian and, at the same time, including some projectors at the boundary. This gives us another description of the second boundary of the CZX model (see Fig. 25). The bulk Hamiltonian of the model is still given by $H_{p}$ for each complete octagon in the bulk, with addition terms that force the boundary spin $\sigma_{i_{ \pm}}$'s to have the same $\sigma^{z}$ value as the bulk spins connected by the green lines. However, notice that the shaded squares are not complete octagons, since the two spins to the right of the shaded squares do not need to be parallel. So, the Hamiltonian for the shaded squares needs to be modified:

$H_{p}^{\text {shaded }}=-H_{p}^{0} P_{p}^{u} P_{p}^{d} P_{p}^{l} P_{p}^{r}+\tilde{H}_{p}^{0} P_{p}^{u} P_{p}^{d} P_{p}^{l}\left(1-P_{p}^{r}\right)$, 


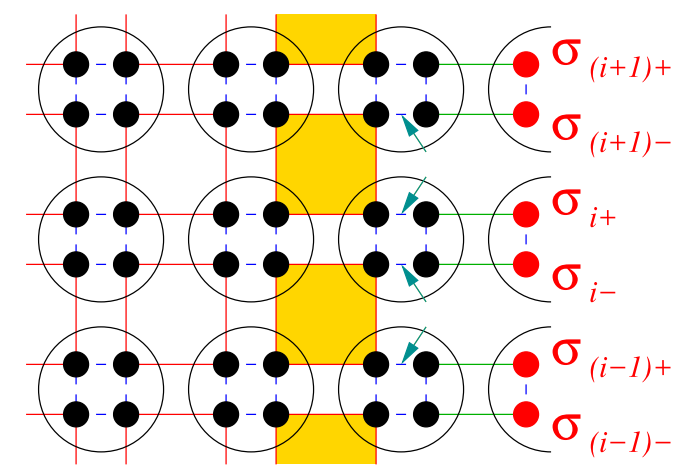

FIG. 25. The filled dots are qubits (or spin-1/2's). A circle (with dots inside) represents a site. The bulk Hamiltonian contains terms that force the dots connected by red and green lines to have the same $\sigma_{i}^{z}$ at low energies. The dashed blue line connecting dots $i, j$ represents the phase factor $C Z_{i j}$ in the bulk $Z_{2}^{G}$ global symmetry transformation.

where $\tilde{H}_{p}^{0}$ is given by

$$
\tilde{H}_{p}^{0}=\mathrm{i}(|\downarrow \downarrow \downarrow \downarrow\rangle\langle\uparrow \uparrow \uparrow \uparrow|-| \uparrow \uparrow \uparrow \uparrow\rangle\langle\downarrow \downarrow \downarrow \downarrow|) .
$$

The above Hamiltonian has a $Z_{4} \equiv Z_{4}^{H}$ global symmetry. The $Z_{4}^{H}$-symmetry is generated by

$$
\sigma_{i_{-}}^{x} \sigma_{i_{+}}^{x} U_{C Z, i_{-}, i_{+}},
$$

when it acts on a boundary site, and by

$U_{X, s} U_{C Z, s}=\sigma_{i_{1}}^{x} \sigma_{i_{2}}^{x} \sigma_{i_{3}}^{x} \sigma_{i_{4}}^{x} U_{C Z, i_{1}, i_{2}} U_{C Z, i_{2}, i_{3}} U_{C Z, i_{3}, i_{4}} U_{C Z, i_{4}, i_{1}}$,

when it acts on a bulk site, where $i_{1}, i_{2}, i_{3}$, and $i_{4}$ label the four spins on the bulk site. Note that the $Z_{4}$-symmetry is actually a $Z_{2}$-symmetry in the bulk, since

$$
\left(U_{X, s} U_{C Z, s}\right)^{2}=1 .
$$

So here, we are actually considering a model with on-site $Z_{2}^{G}$-symmetry in the bulk, and the symmetry is promoted to $Z_{4}^{H}$-symmetry on the boundary, since

$$
\left(\sigma_{i_{-}}^{x} \sigma_{i_{+}}^{x} U_{C Z, i_{-}, i_{+}}\right)^{2}=-\sigma_{i_{-}}^{z} \sigma_{i_{+}}^{z} \neq 1 .
$$

The total symmetry generator is given by

$$
\hat{U}_{Z_{4}}=\prod_{i} \sigma_{i_{-}}^{x} \sigma_{i_{+}}^{x} U_{C Z, i_{-}, i_{+}} \prod_{\text {bulk sites } s} U_{X, s} U_{C Z, s} .
$$

To see that $H_{p}^{\text {shaded }}$ is invariant under $\hat{U}_{Z_{4}}$, we first note that $H_{p}^{0} P_{p}^{u} P_{p}^{d} P_{p}^{l} P_{p}^{r}$ is invariant under $\hat{U}_{Z_{4}}$. Rewriting $\tilde{H}_{p}^{0} P_{p}^{u} P_{p}^{d} P_{p}^{l}\left(1-P_{p}^{r}\right)$ as $\mathrm{i} H_{p}^{0} P_{p}^{u} P_{p}^{d} P_{p}^{l}\left(1-P_{p}^{r}\right) \sigma_{i_{1}}^{z}$, we see that $\sigma_{i_{1}}^{z}$ anticommutes with $\hat{U}_{Z_{4}} \cdot H_{p}^{0} P_{p}^{u} P_{p}^{d} P_{p}^{l}\left(1-P_{p}^{r}\right)$ also anticommutes with $\hat{U}_{Z_{4}}$. Thus, $H_{p}^{\text {shaded }}$ is invariant under $\hat{U}_{Z_{4}}$.

The low-energy boundary excitations have a basis labeled by $\sigma_{i_{ \pm}}^{z}$ values of the boundary spins:

$$
\left.\left|\left\{\sigma_{i_{ \pm}}^{z}\right\}\right\rangle_{\text {whole }}=\left|\left\{\sigma_{i_{ \pm}}^{z}\right\}\right\rangle_{\text {bdry }} \times \mid \text { bulk }\right\rangle .
$$

Now, $\mid$ bulk $\rangle$ is given by

$\mid$ bulk $\rangle=\bigotimes_{\text {squares }} \mid$ square $\rangle \bigotimes_{\text {shaded-squares }} \mid$ shaded-square $\rangle$,

where $\mid$ square $\rangle \equiv(1 / \sqrt{2})(|\uparrow \uparrow \uparrow \uparrow\rangle+|\downarrow \downarrow \downarrow \downarrow\rangle)$ is the spin state for the four spins connected by a red square in Fig. 25, as determined by $H_{p}$, and

$$
\begin{aligned}
& \mid \text { shaded-square }\rangle \equiv \frac{|\uparrow \uparrow \uparrow \uparrow\rangle+|\downarrow \downarrow \downarrow \downarrow\rangle}{\sqrt{2}} \text { if } \sigma_{i_{+}}^{z} \sigma_{(i+1)_{-}}^{z}=1, \\
& \mid \text { shaded-square }\rangle \equiv \frac{|\uparrow \uparrow \uparrow \uparrow\rangle-\mathrm{i}|\downarrow \downarrow \downarrow \downarrow\rangle}{\sqrt{2}} \text { if } \sigma_{i_{+}}^{z} \sigma_{(i+1)_{-}}^{z}=-1,
\end{aligned}
$$

is the spin state for the four spins connected by a shaded red square in Fig. 25, as determined by $H_{p}^{\text {bdry }}$.

Under the $\hat{U}_{Z_{4}},(|\uparrow \uparrow \uparrow \uparrow\rangle+|\downarrow \downarrow \downarrow \downarrow\rangle / \sqrt{2})$ is unchanged for $\sigma_{i_{+}}^{z} \sigma_{(i+1)_{-}}^{z}=1$. But for $\sigma_{i_{+}}^{z} \sigma_{(i+1)_{-}}^{z}=-1, \hat{U}_{Z_{4}}$ changes $|\uparrow \uparrow \uparrow \uparrow\rangle \rightarrow|\downarrow \downarrow \downarrow \downarrow\rangle$ and $|\downarrow \downarrow \downarrow \downarrow\rangle \rightarrow-|\uparrow \uparrow \uparrow \uparrow\rangle$. The extra sign comes from the two uncanceled $C Z$ factors to the right of the plaquette (see Fig. 25, where the $C Z$ factors are pointed out by arrows). Therefore, under the $\hat{U}_{Z_{4}}$, $(|\uparrow \uparrow \uparrow \uparrow\rangle-i|\downarrow \downarrow \downarrow \downarrow\rangle / \sqrt{2})$ is changed to

$$
\frac{|\downarrow \downarrow \downarrow \downarrow\rangle+\mathrm{i}|\uparrow \uparrow \uparrow \uparrow \uparrow\rangle}{\sqrt{2}}=\mathrm{i} \frac{|\uparrow \uparrow \uparrow \uparrow\rangle-\mathrm{i}|\downarrow \downarrow \downarrow \downarrow\rangle}{\sqrt{2}} .
$$

So, under the $Z_{4}$ on-site transformation to the whole system, the bulk state $\mid$ bulk $\rangle$ changes into itself up to a phase factor:

$$
\left.\mid \text { bulk }\rangle \rightarrow \mathrm{e}^{\mathrm{i} \theta} \mid \text { bulk }\right\rangle \text {. }
$$

The phase factor $\mathrm{e}^{\mathrm{i} \theta}$ depends on the boundary spins $\sigma_{i}^{z}$ and is given by

$$
\mathrm{e}^{\mathrm{i} \theta}=\prod_{i} \mathrm{i}^{\left(1-\sigma_{i_{+}}^{z} \sigma_{(i+1)-}^{z}\right) / 2} U_{C Z, i_{-}, i_{+}} \cdot
$$

The $C Z_{i_{-}, i_{+}}$factors in Eqs. (A13) and (A3) cancel each other. Therefore, the effective $Z_{4}^{H}$ transformation on the boundary low-energy subspace is given by 


$$
\begin{aligned}
\hat{U}_{Z_{4}} & =\prod_{i} \sigma_{i_{-}}^{x} \sigma_{i_{+}}^{x} \mathrm{i}^{\left(1-\sigma_{i_{+}}^{z} \sigma_{(i+1)_{-}}^{z}\right) / 2} \\
& =\prod_{i} \sigma_{i_{+}}^{x} \sigma_{(i+1)_{-}}^{x} \mathrm{i}^{\left(1-\sigma_{i_{+}}^{z} \sigma_{(i+1)}^{z}\right) / 2},
\end{aligned}
$$

which is an on-site symmetry if we view $\left[i_{+},(i+1)_{-}\right]$as a site. This means that if we view the CZX model as a model with $Z_{4}$-symmetry, it is actually a trivial $H=Z_{4}^{H}$-SPT state (since the effective $Z_{4}^{H}$ transformation on the boundary is on-site and anomaly free).

To summarize, the original model in the Sec. III B describes a gapped boundary, where the boundary plaquette term $H_{p}^{\text {bdry }}$ has the same order as the bulk plaquette term. Now in this Sec. A 1, we reduce the boundary plaquette term $H_{p}^{\text {bdry }}$ to only some newly introduced projectors on the green links in Fig. 25. For certain small or zero $H_{p}^{\text {bdry }}$, the boundary spins may have no constraint in the whole wave function $\left|\left\{\sigma_{i_{ \pm}}^{z}\right\}\right\rangle_{\text {whole }}=\left|\left\{\sigma_{i_{ \pm}}^{z}\right\}\right\rangle_{\text {bdry }} \times \mid$ bulk $\rangle$, which can describe a gapless boundary. We have also obtained the effective $Z_{4}^{H}$-symmetry transformation on the boundary.

\section{The low-energy effective theory for the fourth boundary of the CZX model: A 1+1D exactly soluble emergent $Z_{2}^{K}$-gauge theory}

In the last subsection, we have constructed a boundary of the CZX model that has a $Z_{4}^{H}$-symmetry. In this section, we are going to modify the above construction to obtain a boundary that has the same $Z_{2}^{G}$-symmetry as the bulk. We will obtain a low-energy effective theory for the fourth boundary of the CZX model discussed in Sec. III D.

\section{a. The boundary $Z_{2}^{K}$-gauge theory with an anomalous $Z_{2}^{G}$ global symmetry}

We start with the boundary model obtained in last Sec. A 1 and add qubits described by $\boldsymbol{\tau}_{i_{ \pm}}$(see Fig. 26). However, the boundary physical Hilbert space is the subspace that satisfies a local gauge constraint

$$
\hat{U}_{i}^{\text {gauge }} \equiv-\sigma_{i_{+}}^{z} \sigma_{i_{-}}^{z} \tau_{i_{+}}^{z} \tau_{i_{-}}^{z}=1 .
$$

The symmetry generator is the same as before when acting on $\boldsymbol{\sigma}_{i_{ \pm}}$spins. The symmetry generator acts on the $\boldsymbol{\tau}_{i_{ \pm}}$ spins as

$$
\prod_{i} \mathrm{e}^{\mathrm{i} \frac{\pi}{4} \tau_{i-}^{z}} \mathrm{e}^{-\mathrm{i} \frac{\pi}{4} \tau_{i_{+}}^{z}}
$$

As we have discussed in Sec. IIID, such a symmetry generator generates an on-site global $Z_{2}^{G}$-symmetry, in the $Z_{2}^{K}$-gauge-invariant physical Hilbert space.

Using the effective boundary $Z_{4}^{H}$-symmetry calculated in Appendix A 1 [see Eq. (A14)], plus an additional term

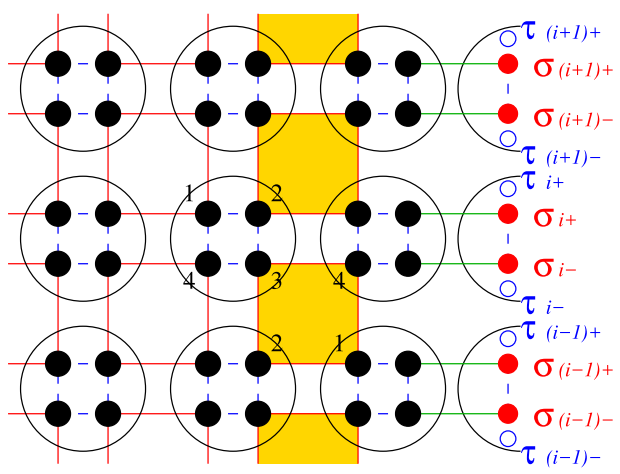

FIG. 26. The filled dots are qubits $\uparrow, \downarrow$ (or spin-1/2's). The open blue dots are qubits \pm 1 representing $Z_{2}^{K}$-gauge degrees of freedom. A circle (with dots inside) represents a bulk site. The bulk Hamiltonian contains terms that force the dots connected by red and green lines to have the same $\sigma_{i}^{z}$ at low energies. The dashed blue line connecting dots $i, j$ represents the phase factor $U_{C Z, i j}$ in the $Z_{2}^{G}$ global symmetry transformation. The open dots on the boundary are the qubits $\boldsymbol{\tau}_{i_{ \pm}}$.

$\mathrm{e}^{\mathrm{i} \frac{\pi}{4} \tau_{i_{-}}^{z}} \mathrm{e}^{-\mathrm{i} \frac{\pi}{4} \tau_{i_{+}}^{z}}$ acting on the new $\tau_{i_{ \pm}}$spins, we find that the boundary effective symmetry generator is given by

$$
\hat{U}_{Z_{2}}=\prod_{i} \sigma_{i_{+}}^{x} \sigma_{(i+1)_{-}}^{x} \mathrm{i}^{\left(1-\sigma_{i_{+}}^{z} \sigma_{(i+1)-}^{z}\right) / 2} \mathrm{e}^{\mathrm{i} \frac{\pi}{4} \tau_{-}^{z}} \mathrm{e}^{-\mathrm{i} \frac{\pi}{4} \tau_{i_{+}}^{z}} .
$$

$\hat{U}_{Z_{2}}$ satisfies

$$
\begin{aligned}
\hat{U}_{Z_{2}}^{2} & =\prod_{i} \sigma_{i_{+}}^{z} \sigma_{(i+1)_{-}}^{z} \mathrm{i} \tau_{i_{-}}^{z}(-\mathrm{i}) \tau_{i_{+}}^{z} \\
& =\prod_{i}\left(-\sigma_{i_{+}}^{z} \sigma_{(i+1)_{-}}^{z} \tau_{i_{-}}^{z} \tau_{i_{+}}^{z}\right) \prod_{i}(-1)=1
\end{aligned}
$$

in the constraint $Z_{2}^{K}$-gauge-invariant subspace. Here, we encounter the even-odd lattice site effect again; we assume that the total number of the boundary sites is always even, $\prod_{i}(-1)=1$, including the example that the whole system is on a disk with only a single boundary. We have turned the $Z_{4}^{H}$-symmetry in the last subsection into a $Z_{2}^{G}$-symmetry.

Next, let us include a boundary interaction term $-U_{\tau} \sum_{i} \tau_{i_{+}}^{z} \tau_{(i+1)_{-}}^{z}$. In the following, we will take the $U_{\tau} \rightarrow$ $+\infty$ limit. In this case, the interaction locks $\tau_{i_{+}}^{z}=\tau_{(i+1)_{-}}^{z}$. In the low-energy subspace, we introduce

$$
E_{i+\frac{1}{2}}=\tau_{i_{+}}^{z}=\tau_{(i+1)_{-}}^{z} \quad V_{i+\frac{1}{2}}=\tau_{i_{+}}^{x} \tau_{(i+1)_{-}}^{x},
$$

that satisfies

$$
E_{i+\frac{1}{2}} V_{i+\frac{1}{2}}=-V_{i+\frac{1}{2}} E_{i+\frac{1}{2}} .
$$

Now the $Z_{2}^{K}$-gauge constraint becomes

$$
-E_{i-\frac{1}{2}} \sigma_{i_{-}}^{z} \sigma_{i_{+}}^{z} E_{i+\frac{1}{2}}=1 .
$$


The effective $Z_{2}^{G}$-symmetry generator becomes

$$
\hat{U}_{Z_{2}}=\prod_{i} \sigma_{i_{+}}^{x} \sigma_{(i+1)_{-}}^{x} \mathrm{i}^{\left(1-\sigma_{i_{+}}^{z} \sigma_{(i+1)_{-}}^{z}\right) / 2}
$$

After obtaining the effective $Z_{2}^{G}$-symmetry on the boundary, we can write down a global $Z_{2}^{G}$-symmetric [under Eq. (A22)] and local $Z_{2}^{K}$-gauge-symmetric [under Eq. (A21)] boundary effective Hamiltonian:

$$
\begin{aligned}
H= & -\sum_{i} V_{i+\frac{1}{2}}(|\uparrow \uparrow\rangle\langle\downarrow \downarrow|+| \downarrow \downarrow\rangle\langle\uparrow \uparrow|)_{i_{+},(i+1)_{-}} \\
& -J \sum_{i} \sigma_{i_{+}}^{z} \sigma_{(i+1)_{-}}^{z}-U \sum_{i} E_{i+\frac{1}{2}} \\
= & -\sum_{i} V_{i+\frac{1}{2}}\left(\sigma_{i_{+}}^{+} \sigma_{(i+1)_{-}}^{+}+\sigma_{i_{+}}^{-} \sigma_{(i+1)_{-}}^{-}\right) \\
& -J \sum_{i} \sigma_{i_{+}}^{z} \sigma_{(i+1)_{-}}^{z}-U \sum_{i} E_{i+\frac{1}{2}} .
\end{aligned}
$$

This is our fourth boundary of the CZX model discussed in Sec. III D, but now it becomes a $1+1 \mathrm{D}$ lattice $Z_{2}^{K}$-gauge theory with an anomalous (non-on-site) global $Z_{2}^{G}$-symmetry.

\section{b. Confined $Z_{2}^{K}$-gauge state: A spontaneous symmetry-breaking state}

In general, a large $U$ in the above Hamiltonian will give us a $Z_{2}^{K}$-gauge confined phase (which will be discussed later in more detail). In the $Z_{2}^{K}$-gauge confined phase induced by a large $U$, we have $E_{i+\frac{1}{2}}=1$. In this case, because of Eqs. (A19) and (A21), $\sigma_{i_{-}}^{z} \sigma_{i_{+}}^{z}=-1$ on every site, which reduces two spin $\sigma_{i_{-}}$and $\sigma_{i_{+}}$into one spin $\sigma_{i}$. This reduces the $Z_{2}^{G}$-symmetry transformation into

$$
\hat{U}_{Z_{2}}=\prod_{i} \tilde{\sigma}_{i}^{x} \prod_{i} \mathrm{i}^{\left(1-\left(\tilde{\sigma}_{i}^{z}\right)\left(-\tilde{\sigma}_{i+1}^{z}\right)\right) / 2}
$$

which is a non-on-site (anomalous) $Z_{2}^{G}$-symmetry transformation. Here, $\tilde{\sigma}_{i}^{x}$ is a redefinition of $\sigma_{i_{-}}^{x} \sigma_{i_{+}}^{x}$ for the composite spin. More precisely, because of the gauge constraint $\sigma_{i_{-}}^{z} \sigma_{i_{+}}^{z}=-1$, $\tilde{\sigma}_{i}^{x}$ flips the composite spin as $\left.\tilde{\sigma}_{i}^{x}|\uparrow\rangle_{i_{-}}\left|\downarrow_{i_{+}}=\right| \downarrow\right\rangle_{i_{-}}|\uparrow\rangle_{i_{+}} \quad$ and $\quad \tilde{\sigma}_{i}^{x}|\downarrow\rangle_{i_{-}}|\uparrow\rangle_{i_{+}}=|\uparrow\rangle_{i_{-}}|\downarrow\rangle_{i_{+}}$. Since the two spins are locked, $\sigma_{i_{-}}^{z} \sigma_{i_{+}}^{z}=-1$, in the same site, we can also simply define $\tilde{\sigma}_{i}^{z} \equiv \sigma_{i_{+}}^{z}$, so that $\tilde{\sigma}_{i+1}^{z} \equiv \sigma_{(i+1)_{+}}^{z}=-\sigma_{(i+1)_{-}}^{z}$. So in the large $U$ limit, the lattice $Z_{2}^{K}$-gauge theory, at low energies, reduces to the boundary of the CZX model constructed in Sec. III A. When $J>0$, the confined $Z_{2}^{K}$-gauge state is a ferromagnetic state that spontaneously breaks the global $Z_{2}^{G}-$ symmetry.

\section{c. Deconfined $Z_{2}^{K}$-gauge state in $1+1 D$}

The model in Eq. (A23) is exactly soluble. This is because, in the big Hilbert space before projecting into the $Z_{2}^{K}$-gauge-invariant subspace, the Hamiltonian $H$ in Eq. (A23) is a sum of nonoverlapping local terms: $H=\sum_{i} H_{i, i+1}$, with

$$
\begin{aligned}
H_{i, i+1}= & -V_{i+\frac{1}{2}}\left[\sigma_{i_{+}}^{+} \sigma_{(i+1)_{-}}^{+}+\sigma_{i_{+}}^{-} \sigma_{(i+1)_{-}}^{-}\right] \\
& -J \sigma_{i_{+}}^{z} \sigma_{(i+1)_{-}}^{z}-U E_{i+\frac{1}{2}} .
\end{aligned}
$$

So, the energy spectrum of $H$ can be obtained exactly from that of $H_{i, i+1}$. The $Z_{2}^{K}$-gauge transformation

$$
\hat{U}_{i}^{\text {gauge }}=-\left(E_{i-\frac{1}{2}} \sigma_{i_{-}}^{z}\right)\left(\sigma_{i_{+}}^{z} E_{i+\frac{1}{2}}\right)
$$

commutes with $H$. So, the energy spectrum of $H$ in the $Z_{2}^{K}$ gauge-invariant subspace is a subset of the spectrum in the big unconstrained Hilbert space.

In the deconfined state at $U=J=0, V_{i+\frac{1}{2}}= \pm 1$ and does not fluctuate before we apply the $Z_{2}^{K}$-gauge constraint (i.e., $V_{i+\frac{1}{2}}$ does not fluctuate in the big Hilbert space before projecting into the $Z_{2}^{K}$-gauge-invariant subspace, since $\left.\left[V_{i+\frac{1}{2}}, H\right]=0\right)$. The ground-state wave function on each link is $\left(|\uparrow \uparrow\rangle+v_{i+\frac{1}{2}}|\downarrow \downarrow\rangle\right)_{i_{+},(i+1)_{-}} \otimes\left|v_{i+\frac{1}{2}}\right\rangle$, where $\mid v_{i+\frac{1}{2}}=$ $\pm 1\rangle$ are the eigenstates of $V_{i+\frac{1}{2}}$. The gauge-invariant ground states $\left|\Psi_{\mathrm{gs}}( \pm)\right\rangle$ are two distinct holonomy sectors labeled by $\prod_{i} v_{i+\frac{1}{2}}= \pm 1$, explicitly as

$$
\begin{aligned}
\left|\Psi_{\mathrm{gs}}( \pm)\right\rangle & =\sum_{\left\{v_{i+\frac{1}{2}}\right\}, \prod_{i} v_{i+\frac{1}{2}}= \pm 1} c_{\left\{v_{i+\frac{1}{2}}\right\}} \otimes\left(|\uparrow \uparrow\rangle+v_{i+\frac{1}{2}}|\downarrow \downarrow\rangle\right)_{i_{+},(i+1)_{-}} \\
& \otimes\left|v_{i+\frac{1}{2}}\right\rangle .
\end{aligned}
$$

Here, the coefficient $c_{\left\{v_{i+\frac{1}{2}}\right\}}$ is determined in the same way as Eqs. (3.15) and (3.16) with alternating \pm 1 signs set by the gauge-invariant constraint on the ground states $\left|\Psi_{\text {gs }}( \pm)\right\rangle$.

Under the $\hat{U}_{Z_{2}}$ global symmetry operation in Eq. (A22),

$$
|\uparrow \uparrow\rangle+v_{i+\frac{1}{2}}|\downarrow \downarrow\rangle \rightarrow v_{i+\frac{1}{2}}\left(|\uparrow \uparrow\rangle+v_{i+\frac{1}{2}}|\downarrow \downarrow\rangle\right) .
$$

Thus,

$$
\hat{U}_{Z_{2}}\left|\Psi_{\mathrm{gs}}( \pm)\right\rangle=\prod_{i}\left(v_{i+\frac{1}{2}}\right)\left|\Psi_{\mathrm{gs}}( \pm)\right\rangle
$$

From the above results, we see that the global $Z_{2}^{G}$ charge and the $Z_{2}^{K}$-gauge flux $\prod_{i} v_{i+\frac{1}{2}}$ are locked. In other words, the deconfined state has two degenerate ground states on the ring and a finite energy gap. One ground state carries the global $Z_{2}^{G}$ charge 0 and no $Z_{2}^{K}$-gauge flux through the ring. The other carries the global $Z_{2}^{G}$ charge 1 and the $\pi Z_{2}^{K}$ gauge flux through the ring. Near the end of the next section, 
we will show that the above deconfined states spontaneously break the global $Z_{2}^{G}$-symmetry, which is another way to understand the two degenerate ground states on the ring.

\section{d. Deconfined and confined $Z_{2}^{K}$-gauge states belong to the same phase that spontaneously breaks the $Z_{2}^{G}$ global symmetry}

We note that, for the following four spin states, $|\uparrow \uparrow\rangle+|\downarrow \downarrow\rangle,|\uparrow \uparrow\rangle-|\downarrow \downarrow\rangle,|\uparrow \downarrow\rangle$, and $|\downarrow \uparrow\rangle$, are common eigenstates of $\sigma_{i_{+}}^{+} \sigma_{(i+1)_{-}}^{+}+\sigma_{i_{+}}^{-} \sigma_{(i+1)_{-}}^{-}$and $\sigma_{i_{+}}^{z} \sigma_{(i+1)_{-}}^{z}$ with eigenvalues $(1,1) ;(-1,1) ;(0,-1)$; and $(0,-1)$.

For $U, J>0$, the ground states have a twofold degeneracy, which is given by

$$
\begin{aligned}
& \left|\psi_{1}\right\rangle=(|\uparrow \uparrow\rangle+|\downarrow \downarrow\rangle)_{i_{+},(i+1)_{-}} \otimes[\cos (\theta)|1\rangle+\sin (\theta)|-1\rangle]_{i+\frac{1}{2}}, \\
& \left|\psi_{2}\right\rangle=(|\uparrow \uparrow\rangle-|\downarrow \downarrow\rangle)_{i_{+},(i+1)_{-}} \otimes[\sin (\theta)|1\rangle+\cos (\theta)|-1\rangle]_{i+\frac{1}{2}},
\end{aligned}
$$

where $| \pm 1\rangle$ are eigenstates of $V_{i+\frac{1}{2}}$ with eigenvalues \pm 1 . In order to have the two states as ground states, $\theta$ is constrained to be the function of $U$ as $\theta=\frac{1}{2} \tan ^{-1} U$.

The energy of the two ground states is $E=-\sqrt{1+U^{2}}-$ $J$. Also, $\theta=0$ for $U=0$ (the $Z_{2}^{K}$-gauge deconfined case) and $\theta \rightarrow \pi / 4$ for $U \rightarrow+\infty$ (the $Z_{2}^{K}$-gauge confined case). The first excited states also have a twofold degeneracy, which is given by

$$
\begin{aligned}
&|\uparrow \downarrow\rangle_{i_{+},(i+1)_{-}} \otimes(|1\rangle+|-1\rangle)_{i+\frac{1}{2}}, \\
& \text { and } \quad|\downarrow \uparrow\rangle_{i_{+},(i+1)_{-}} \otimes(|1\rangle+|-1\rangle)_{i+\frac{1}{2}},
\end{aligned}
$$

with energy $E=-|U|+J$, which is higher than the ground-state energy by at least $2 J$ (note that we have assumed $J>0$ ).

We note that

$$
\begin{aligned}
(|\uparrow \uparrow\rangle+|\downarrow \downarrow\rangle) & \otimes[\cos (\theta)|1\rangle+\sin (\theta)|-1\rangle] \\
+(|\uparrow \uparrow\rangle-|\downarrow \downarrow\rangle) & \otimes[\sin (\theta)|1\rangle+\cos (\theta)|-1\rangle] \\
& \equiv|++\rangle
\end{aligned}
$$

is a common eigenstate of $\left(\sigma_{i_{+}}^{z} E_{i+\frac{1}{2}}, E_{i+\frac{1}{2}} \sigma_{(i+1)_{-}}^{z}\right)$ with eigenvalues $(+1,+1)$, and we denote it as $|++\rangle$ or $|++\rangle_{i_{+}, i+\frac{1}{2},(i+1)_{-}}$. Similarly,

$$
\begin{aligned}
(|\uparrow \uparrow\rangle+|\downarrow \downarrow\rangle) & \otimes(\cos (\theta)|1\rangle+\sin (\theta)|-1\rangle) \\
-(|\uparrow \uparrow\rangle-|\downarrow \downarrow\rangle) & \otimes(\sin (\theta)|1\rangle+\cos (\theta)|-1\rangle) \\
& \equiv|--\rangle
\end{aligned}
$$

is a common eigenstate of $\left(\sigma_{i_{+}}^{z} E_{i+\frac{1}{2}}, E_{i+\frac{1}{2}} \sigma_{(i+1)_{-}}^{z}\right)$ with eigenvalues $(-1,-1)$, and we denote it as $|--\rangle$ or $|--\rangle_{i_{+}, i+\frac{1}{2},(i+1)_{-}}$.
A $Z_{2}^{K}$-gauge-invariant ground state (i.e., $\hat{U}_{i}^{\text {gauge }}=1$ state) on a ring is given by the tensor product of those $|++\rangle$ and $|--\rangle$ states on the $(i, i+1)$ links. First, we note that the gauge transformation in Eq. (A26) is a product of two operators $E_{i-\frac{1}{2}} \sigma_{i_{-}}^{z}$ and $\sigma_{i_{+}}^{z} E_{i+\frac{1}{2}}$ with an additional - sign. The $|++\rangle$ and $|--\rangle$ are eigenstates of those operators. Therefore, we have two $Z_{2}^{K}$-gauge-invariant ground states:

$$
\begin{aligned}
\left|\Psi_{1}(\theta)\right\rangle= & \cdots \otimes|++\rangle_{(i-1)_{+}, i-\frac{1}{2}, i_{-}} \otimes|--\rangle_{i_{+}, i+\frac{1}{2},(i+1)_{-}} \\
& \otimes|++\rangle_{(i+1)_{+}, i+\frac{3}{2},(i+2)_{-}} \otimes \cdots, \\
\left|\Psi_{2}(\theta)\right\rangle= & \cdots \otimes|--\rangle_{(i-1)_{+}, i-\frac{1}{2}, i_{-}} \otimes|++\rangle_{i_{+}, i+\frac{1}{2},(i+1)_{-}} \\
& \otimes|--\rangle_{(i+1)_{+}, i+\frac{3}{2},(i+2)_{-}} \otimes \cdots,
\end{aligned}
$$

up to a proper normalization factor. Note that, to get a $Z_{2}^{K}$-gauge-invariant state under Eq. (A26), we need to match + to - and - to + in the neighboring links, as done in the above. However, the two ground states expressed in Eq. (A34) are not symmetric under the global $Z_{2}^{G}$-symmetry transformation in Eq. (A22):

$$
\hat{U}_{Z_{2}}=\prod_{i} \sigma_{i_{+}}^{x} \sigma_{(i+1)_{-}}^{x} \mathrm{i}^{\left(1-\sigma_{i_{+}}^{z} \sigma_{(i+1)_{-}}^{z}\right) / 2} \equiv \prod_{i} U_{Z_{2}, i_{+},(i+1)_{-}}
$$

In fact, $U_{Z_{2}, i_{+},(i+1)_{-}}$exchanges $|++\rangle$and $|--\rangle$,

$U_{Z_{2}, i_{+},(i+1)_{-}}|++\rangle_{(i-1)_{+}, i-\frac{1}{2}, i_{-}}=|--\rangle_{(i-1)_{+}, i-\frac{1}{2}, i_{-}}$,

$U_{Z_{2}, i_{+},(i+1)_{-}}|--\rangle_{(i-1)_{+}, i-\frac{1}{2}, i_{-}}=|++\rangle_{(i-1)_{+}, i-\frac{1}{2}, i_{-}}$.

The ground states that respect the global $Z_{2}^{G}$-symmetry transformation in Eq. (A22) are the linear combination of Eq. (A34):

$$
\begin{aligned}
\left|\Psi_{\mathrm{gs}, \text { even }}(\theta)\right\rangle & =\frac{1}{\sqrt{2}}\left[\left|\Psi_{1}(\theta)\right\rangle+\left|\Psi_{2}(\theta)\right\rangle\right] \\
\left|\Psi_{\mathrm{gs}, \text { odd }}(\theta)\right\rangle & =\frac{1}{\sqrt{2}}\left[\left|\Psi_{1}(\theta)\right\rangle-\left|\Psi_{2}(\theta)\right\rangle\right],
\end{aligned}
$$

where the $\left|\Psi_{\mathrm{gs}, \text { even }}(\theta)\right\rangle$ is $Z_{2}^{G}$-symmetry even by $\hat{U}_{Z_{2}}\left|\Psi_{\text {gs,even }}(\theta)\right\rangle=+\left|\Psi_{\text {gs,even }}(\theta)\right\rangle$, and the $\left|\Psi_{\text {gs odd }}(\theta)\right\rangle$ is $Z_{2}^{G}$-symmetry odd by $\hat{U}_{Z_{2}}\left|\Psi_{\mathrm{gs}, \text { odd }}(\theta)\right\rangle=-\left|\Psi_{\mathrm{gs}, \text { odd }}(\theta)\right\rangle$.

When $\theta=0$, the even or odd $Z_{2}^{G}$-symmetric ground states are identical to the even or odd $Z_{2}^{K}$-gauge holonomy sectors of ground states in Eq. (A27) because of the locking of $Z_{2}^{G}$-charge and $Z_{2}^{K}$-holonomy: 


$$
\begin{gathered}
\left|\Psi_{\mathrm{gs}, \mathrm{even}}(\theta=0)\right\rangle=\frac{1}{\sqrt{2}}\left[\left|\Psi_{1}(0)\right\rangle+\left|\Psi_{2}(0)\right\rangle\right]=\left|\Psi_{\mathrm{gs}}(+)\right\rangle, \\
\left|\Psi_{\mathrm{gs}, \mathrm{odd}}(\theta=0)\right\rangle=\frac{1}{\sqrt{2}}\left[\left|\Psi_{1}(0)\right\rangle-\left|\Psi_{2}(0)\right\rangle\right]=\left|\Psi_{\mathrm{gs}}(-)\right\rangle .
\end{gathered}
$$

When $\theta=(\pi / 4)$, we have the confined states:

$$
\begin{aligned}
\left|\Psi_{1}\left(\theta=\frac{\pi}{4}\right)\right\rangle= & \left(\cdots \otimes|\uparrow \uparrow\rangle_{(i-1)_{+}, i_{-}} \otimes|\downarrow \downarrow\rangle_{i_{+},(i+1)_{-}}\right. \\
& \left.\otimes|\uparrow \uparrow\rangle_{(i+1)_{+},(i+2)_{-}} \otimes \cdots\right) \underset{i}{\otimes}(|1\rangle+|-1\rangle)_{i+\frac{1}{2}}, \\
\left|\Psi_{2}\left(\theta=\frac{\pi}{4}\right)\right\rangle= & \left(\cdots \otimes|\downarrow \downarrow\rangle_{(i-1)_{+}, i_{-}} \otimes|\uparrow \uparrow\rangle_{i_{+},(i+1)_{-}}\right. \\
& \left.\otimes|\downarrow \downarrow\rangle_{(i+1)_{+},(i+2)_{-}} \otimes \cdots\right) \underset{i}{\otimes}(|1\rangle+|-1\rangle)_{i+\frac{1}{2}},
\end{aligned}
$$

up to a proper normalization factor. Below, we aim to show that, at $\theta=0$, namely, $U=0$ and $J>0$, we have the deconfined state with spontaneous $Z_{2}^{G}$-symmetry breaking; at $\theta=(\pi / 4)$, namely, $U \rightarrow+\infty$ and $J>0$, we have the confined state with spontaneous $Z_{2}^{G}$-symmetry breaking. We demonstrate a strange property for this system: The deconfined state with spontaneous $Z_{2}^{G}$-symmetry breaking and the confined state with spontaneous $Z_{2}^{G}$-symmetry breaking belong to the same phase. In the next few paragraphs, we explain the meanings of the deconfined and confined phases, and also the meanings of the spontaneous symmetry breaking.

First, we elaborate further on the physical meanings of the deconfined and confined phases. The deconfined phase $(U=0)$ here means that the distinct holonomies or loop excitations (namely, Wilson lines) can span the large system without causing extra energy. Consider the expectation value $\langle 0|W| 0\rangle$ of Wilson line operator $W \equiv \prod_{i} V_{i+\frac{1}{2}}$ for some ground state $|0\rangle$; the $\langle 0|W| 0\rangle$ goes to some constant (proportional to the net holonomy $\prod_{i} v_{i+\frac{1}{2}}= \pm 1$ ) in the Euclidean spacetime and, thus, obeys the perimeter law instead of the area law [88]. The two ground states with distinct holonomies in our case imply that we are in the deconfined phase, even if the energy spectrum is gapped between the ground states and first excitations. On the other hand, the confined phase ( $U \rightarrow \infty, J>0$ ) has the gauge field variable $\left|v_{i+\frac{1}{2}}\right\rangle$ quantum disorder and strong fluctuations in the state $(|1\rangle+|-1\rangle)_{i+\frac{1}{2}}$. The long-distance lines or holonomies are energy disfavored. Consider the expectation value $\langle 0|W| 0\rangle$ of Wilson line operator $W$ for any ground state $|0\rangle$; the $\langle 0|W| 0\rangle$ exponentially decays to zero in the Euclidean spacetime and, thus, obeys the area law. Therefore, the phase is confined. The $Z_{2}^{K}$-gauge confined phase for $U \rightarrow+\infty$ and $J>0$ is a ferromagnetic along the link $\overline{i_{+}(i+1)_{-}}$but antiferromagnetic between the neighbored links between spin up and down. There is no phase transition as $U$ goes from 0 to $+\infty$ for $J>0$, since the energy gap above the ground state is always bigger than $2 J$. Thus, the $Z_{2}^{K}$-gauge deconfined state for $U=0$ and the $Z_{2}^{K}$ gauge confined state for $U=+\infty$ belong to the same phase.

Second, we elaborate further on the physical meanings of the spontaneous symmetry breaking (SSB) and possible long-range orders. Based on Ref. [89], we know that the SSB in a quantum system does not necessarily mean that its ground states break the symmetry. Traditionally, we identify the symmetry-breaking order parameter and we compute the long-range order correlation functions to detect the symmetry breaking. The better definition for SSB is based on the Greenberger-Horne-Zeilinger (GHZ) entanglement [90]. Using GHZ form, we can probe the symmetry without knowing the symmetry or the Ginzburg-Landau symmetrybreaking order parameters. Using GHZ form, we can detect the symmetry-breaking hidden in the symmetric groundstate wave function.

Indeed, $\left|\Psi_{1}(\theta)\right\rangle$ and $\left|\Psi_{2}(\theta)\right\rangle$ are GHZ states,

$$
\begin{aligned}
\left|\Psi_{\mathrm{gs}, \text { even }}(\theta)\right\rangle & =\frac{1}{\sqrt{2}}\left[\left|\Psi_{1}(\theta)\right\rangle+\left|\Psi_{2}(\theta)\right\rangle\right] \equiv\left|\mathrm{GHZ}_{+}(\theta)\right\rangle \\
\left|\Psi_{\mathrm{gs}, \text { odd }}(\theta)\right\rangle & =\frac{1}{\sqrt{2}}\left[\left|\Psi_{1}(\theta)\right\rangle-\left|\Psi_{2}(\theta)\right\rangle\right] \equiv\left|\mathrm{GHZ}_{-}(\theta)\right\rangle .
\end{aligned}
$$

Because the $Z_{2}^{G}$-global symmetry operator $\hat{U}_{Z_{2}}$ acting on two states gives rise to the symmetric charge \pm 1 , the following conditions for SSB of symmetry group $G$ are satisfied:

(1) $\hat{U}_{Z_{2}}\left|\mathrm{GHZ}_{ \pm}(\theta)\right\rangle= \pm\left|\mathrm{GHZ}_{ \pm}(\theta)\right\rangle$.

(2) The symmetric GHZ states have the same GHZ entanglement $|\mathrm{GHZ}\rangle=\sum_{j} c_{j}\left|\Psi_{j}\right\rangle$, with $j \in G / G^{\prime}$, $G^{\prime} \subset G$, where $\left|\Psi_{j}\right\rangle$ are locally distinguishable. In our case, we have $G=Z_{2}$ and $G^{\prime}$ is trivial.

To summarize, the symmetric many-body state has spontaneous symmetry breaking, which implies that the state has a GHZ entanglement. Indeed, we can also show that the SSB here also implies the long-range order, consistent with what we observed in Eq. (3.21) in Sec. III C. Defining the gauge-invariant operator $X_{i+1 / 2}=\sigma_{i+}^{z} E_{i+1 / 2}$, which is odd, breaking the $Z_{2}^{G}$-symmetry, we find $X_{i+1 / 2}\left|\Psi_{1}(\theta)\right\rangle=-\left|\Psi_{1}(\theta)\right\rangle$ and $X_{i+1 / 2}\left|\Psi_{2}(\theta)\right\rangle=+\left|\Psi_{2}(\theta)\right\rangle$. Moreover,

$$
\left\langle\mathrm{GHZ}_{ \pm}(\theta)\left|X_{i+1 / 2} X_{j+1 / 2}\right| \mathrm{GHZ}_{ \pm}(\theta)\right\rangle=1 .
$$

Thus, the $G$-symmetry odd operator detects the long-range correlator of GHZ states, and we demonstrate the SSB through the long-range order. In summary, we show that the deconfined state and the confined state belong to the same phase without the phase transition by tuning the Hamiltonian 
coupling $U$ with the ground-state parameter $\theta=\frac{1}{2} \tan ^{-1} U$. All values of $U$ have the spontaneous $Z_{2}^{G}$-symmetry breaking. This is possible since the $Z_{2}^{K}$-gauge deconfined phase with no spin order has twofold degenerate ground states with opposite global $Z_{2}^{G}$ charge, the same as the ferromagnetic state with spin order, which also has twofold degenerate ground states with opposite global $Z_{2}^{G}$ charges.

We remind the readers that the fermionic version of the CZX model is studied in Appendix B. The boundary of the fermionic CZX model with emergent $Z_{2}^{K}$-gauge theory with anomalous global symmetry is detailed in Appendix C.

One can read Sec. IV on more general boundaries of SPTs in any dimension.

\section{APPENDIX B: FERMIONIC CZX MODEL}

Consider a square lattice model with each single site endowed with four fermion orbitals, each with eigenstates $|0\rangle$ and $|1\rangle$ of the fermion number operator $n_{f}=c^{\dagger} c$. Thus, a single site has a $2^{4}$-dimensional Hilbert space. We may call the single site a "vertex" and the four individual fermion orbitals in a site "subvertices." In the fermionic model, we have the anticommutation relation

$$
\left\{c_{i}, c_{j}^{\dagger}\right\}=\delta_{i j},
$$

where $i, j$ can be any local fermion degree of freedom, on the same site or on different sites. The fermion parity operator $P_{f}$ on each site (with $1,2,3,4$ as the four subvertices) is

$$
P_{f}=\prod_{i=1,2,3,4}(-1)^{n_{f, i}}=\prod_{j=1,2,3,4} \sigma_{j}^{z} .
$$

Notice that

$$
\left(1-2 c_{i}^{\dagger} c_{i}\right)=\sigma_{j}^{z}, \quad c_{i}^{\dagger} c_{i}=\frac{1-\sigma_{j}^{z}}{2} .
$$

Let us introduce a $Z_{2}$ generator $U_{X}$ as a product of $c_{j}^{\dagger}+c_{j}$ on the four subvertices:

$$
\begin{aligned}
U_{X}= & \left(c_{1}^{\dagger}+c_{1}\right)(-1)^{n_{1}}\left(c_{2}^{\dagger}+c_{2}\right)(-1)^{n_{1}}(-1)^{n_{2}}\left(c_{3}^{\dagger}+c_{3}\right) \\
& \times(-1)^{n_{1}}(-1)^{n_{2}}(-1)^{n_{3}}\left(c_{4}^{\dagger}+c_{4}\right) \\
= & \sigma_{1}^{x} \sigma_{2}^{x} \sigma_{3}^{x} \sigma_{4}^{x}, \quad U_{X}^{2}=1,
\end{aligned}
$$

where we have used the Jordan-Wiger transformation to express fermion operators in terms of spin operators, for example,

$$
c_{j}^{\dagger}+c_{j}=\left(\prod_{i<j} \sigma_{i}^{z}\right) \sigma_{j}^{x},
$$

where $i<j$ refers to a particular ordering of the orbitals (see Fig. 27). We have chosen an unusual

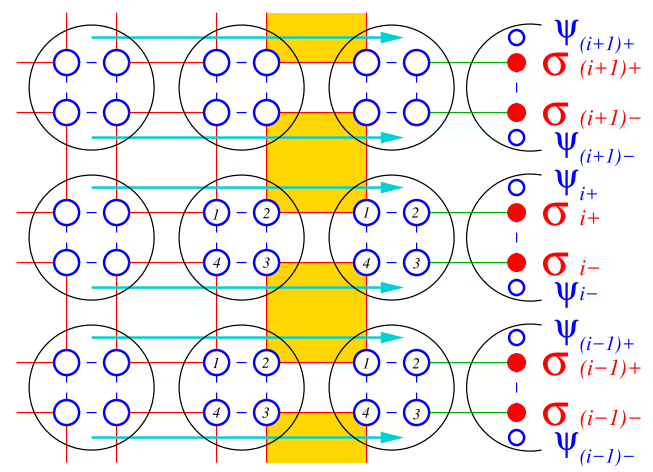

FIG. 27. The filled dots are qubits (or spin-1/2's) described by $\sigma$. The open dots are fermion orbitals described by $c$ or $\psi$. A circle (with dots inside) represents a site. The bulk Hamiltonian contains terms that force the dots connected by red and green lines to have the same $(-1)^{n_{i}}$ or $\sigma_{i}^{z}$ at low energies. The dashed blue line connecting dots $i, j$ represents the phase factor $C Z_{i j}$ in the $Z_{2}^{G}$ global symmetry transformation. The arrow describes a particular ordering of all fermion orbitals.

definition of $U_{X}$ [instead of the more obvious $\left.\left(c_{1}^{\dagger}+c_{1}\right)\left(c_{2}^{\dagger}+c_{2}\right)\left(c_{3}^{\dagger}+c_{3}\right)\left(c_{4}^{\dagger}+c_{4}\right)\right]$, because we want $U_{X}$ to have a simple form after bosonization.

For any pair of qubits, we set $C Z=|00\rangle\langle 00|+| 01\rangle\langle 01|+$ $|10\rangle\langle 10|-| 11\rangle\langle 11|=1-2 c c^{\dagger} c^{\prime} c^{\prime \dagger}$. For each site, we define $U_{C Z}$ as the product of such operators over all successive pairs:

$$
\begin{aligned}
U_{C Z} & =\prod_{j=1,2,3,4}\left(1-2 c_{j+1}^{\dagger} c_{j+1} c_{j}^{\dagger} c_{j}\right) \\
& =\prod_{j=1,2,3,4}\left(1-\frac{\left(1-\sigma_{j+1}^{z}\right)\left(1-\sigma_{j}^{z}\right)}{2}\right) \\
& =\prod_{j=1,2,3,4}\left(\frac{\left(1+\sigma_{j+1}^{z}+\sigma_{j}^{z}-\sigma_{j+1}^{z} \sigma_{j}^{z}\right)}{2}\right),
\end{aligned}
$$

where $j=5 \bmod 4=1 \bmod 4$. Now, we introduce a $Z_{2}$ transformation in each site:

$$
U_{\mathrm{CZX}}=U_{X} U_{C Z}, \quad U_{\mathrm{CZX}}^{2}=1 .
$$

The group supercohomology predicts that there are four distinct fermionic SPTs with $G=Z_{2} \times Z_{2}^{f}$ symmetry from $H_{\text {super }}^{3}\left[Z_{2} \times Z_{2}^{f}, U(1)\right]=\mathbb{Z}_{4}$. The model we will first focus on is the one with the second class $\nu=2$ for $\nu \in \mathbb{Z}_{4}$. The full classification for four distinct fermionic SPTs

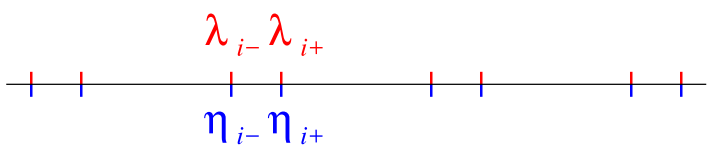

FIG. 28. Emergent $Z_{2}^{K}$-gauge theory from Majorana fermions on the lattice. 
with $Z_{2} \times Z_{2}^{f}$-symmetry is $\mathbb{Z}_{8}$ from the spin cobordism group $\Omega_{3}^{\text {Spin }}\left(B Z_{2}\right)=\mathbb{Z}_{8}$; then, our model here is $\nu=4$ for $\nu \in \mathbb{Z}_{8}$.

The fermionic CZX Hamiltonian is essentially the same as the bosonic CZX Hamiltonian:

$$
\begin{gathered}
H^{f}=\sum H_{p}, \\
H_{p}=-X_{4} P_{2}^{u} P_{2}^{d} P_{2}^{l} P_{2}^{r} .
\end{gathered}
$$

Here, plaquettes are defined in the bosonic CZX model. $X_{4}$ acts on the four subvertices in a plaquette,

$$
\begin{aligned}
X_{4} & =c_{3} c_{4} c_{2} c_{1}+c_{3}^{\dagger} c_{4}^{\dagger} c_{2}^{\dagger} c_{1}^{\dagger} \\
& =\sigma_{4}^{-} \sigma_{3}^{-} \sigma_{2}^{-} \sigma_{1}^{-}+\sigma_{4}^{+} \sigma_{3}^{+} \sigma_{2}^{+} \sigma_{1}^{+} \\
& =(|0000\rangle\langle 1111|+| 1111\rangle\langle 0000|)_{\text {plaquette }},
\end{aligned}
$$

and the projection operator $P_{2}$ acts on a pair of qubits adjacent to a plaquette as

$$
\begin{aligned}
P_{2} & =c_{i} c_{i}^{\dagger} c_{i+1} c_{i+1}^{\dagger}+c_{i}^{\dagger} c_{i} c_{i+1}^{\dagger} c_{i+1} \\
& =(|00\rangle\langle 00|+| 11\rangle\langle 11|)_{\text {line }} .
\end{aligned}
$$

We see that, after bosonization, both the Hamiltonian and the $Z_{2}$-symmetry for the fermionic CZX model map to those of the bosonic CZX model. So, the ground state of the fermionic CZX model is the same as that of the bosonic CZX model described in Sec. II.

It is also obvious that $\left[\prod P_{f}, H_{f}\right]=0$, since $H_{f}$ conserves fermion number mod 2 (in fact, $H_{f}$ conserves fermion number mod 4). So, the fermionic CZX model $H_{f}$ has $Z_{2} \times Z_{2}^{f}$-symmetry generated by $\prod U_{\mathrm{CZX}}$ and $\prod P_{f}$. The ground state is invariant under the symmetry.

\section{APPENDIX C: A BOUNDARY OF THE FERMIONIC CZX MODEL: EMERGENT $Z_{2}^{K}$-GAUGE THEORY WITH AN ANOMALOUS GLOBAL SYMMETRY, AND MAJORANA FERMIONS}

To obtain a boundary of the fermionic CZX model, we start with the boundary model described in Fig. 27. On the boundary, we have qubits described by $\sigma_{i_{+}}$and fermions described by $\psi_{i_{ \pm}}=\eta_{i_{ \pm}}+\mathrm{i} \lambda_{i_{ \pm}}$, where $\eta$ and $\lambda$ are Majorana fermion operators, see Fig. [28].

However, we assume that the boundary Hilbert space is not the one generated by $\sigma_{i_{ \pm}}$and $\psi_{i_{ \pm}}$, but a subspace satisfying a local $Z_{2}^{K}$-gauge constraint:

$$
\hat{U}_{i}^{\text {gauge }}=-\sigma_{i_{+}}^{z} \sigma_{i_{-}}^{z}(-1)^{n_{i_{-}}+n_{i_{+}}}=1,
$$

where

$$
n_{i_{ \pm}}=\psi_{i_{ \pm}}^{\dagger} \psi_{i_{ \pm}} .
$$

Thus, the boundary is a $Z_{2}^{K}$ lattice gauge theory.

The bulk Hamiltonian of the model is still given by $H_{p}^{f}$ for the complete octagons in the bulk, with additional terms that force the boundary qubits $\sigma_{i_{ \pm}}^{z}$ to have the same value as the $(-1)^{n_{i}}$ for the bulk fermions connected by the green lines. However, notice that the shaded squares are not complete octagons, since the two spins to the right of the shaded squares do not need to be parallel. So, the Hamiltonians for the shaded squares need to be modified:

$H_{p}^{f \text {,shaded }}=-X_{4} P_{2}^{u} P_{2}^{d} P_{2}^{l} P_{2}^{r}+\tilde{X}_{4} P_{2}^{u} P_{2}^{d} P_{2}^{l}\left(1-P_{2}^{r}\right)$,

where $\tilde{X}_{4}$ is given in Eq. (A2). The $Z_{2}^{G}$-symmetry of the system is generated by

$\hat{U}_{Z_{2}}=\prod_{i} \sigma_{i_{-}}^{x} \sigma_{i_{+}}^{x} C Z_{i_{-}, i_{+}} \mathrm{e}^{\mathrm{i} \frac{\pi}{4}\left(1-2 n_{i_{-}}\right)} \mathrm{e}^{-\mathrm{i} \frac{\pi}{4}\left(1-2 n_{i_{+}}\right)} \prod_{\text {bulk }} U_{\mathrm{CZX}}$.

After the bosonization via Jordan-Wigner transformation on Majorana fermion operators,

$$
\lambda_{j}=\left(\prod_{i<j} \tau_{i}^{z}\right) \tau_{j}^{x}, \quad \eta_{j}=\left(\prod_{i<j} \tau_{i}^{z}\right) \tau_{j}^{y},
$$

the above Hamiltonian and the $Z_{2}^{G}$-symmetry map to those of the bosonic model discussed in Sec. A 2. So, we can use the results there. First, one can show that

$$
\left(\sigma_{i_{-}}^{x} \sigma_{i_{+}}^{x} C Z_{i_{-}, i_{+}} \mathrm{e}^{\frac{\pi}{4}\left(1-2 n_{i_{-}}\right)} \mathrm{e}^{-\mathrm{i} \frac{\pi}{4}\left(1-2 n_{i_{+}}\right)}\right)^{2}=1
$$

in the $Z_{2}^{K}$-gauge-invariant physical Hilbert space. So, $\hat{U}_{Z_{2}}$ generates an on-site global $Z_{2}^{G}$-symmetry. Second, one can show that the Hamiltonian is indeed $Z_{2}^{G}$ symmetric. Third, one can find the low-energy effective $Z_{2}^{G}$-symmetry on the boundary to be generated by

$$
\hat{U}_{Z_{2}}=\prod_{i} \sigma_{i_{+}}^{x} \sigma_{(i+1)_{-}}^{x} \mathrm{i}^{\left(1-\sigma_{i_{+}}^{z} \sigma_{(i+1)_{-}}^{z}\right) / 2} \mathrm{e}^{\mathrm{i} \frac{\pi}{4}\left(1-2 n_{i_{+}}\right)} \mathrm{e}^{-\mathrm{i} \frac{\pi}{4}\left(1-2 n_{(i+1)_{+}}\right)} .
$$

Next, let us include a boundary interaction term $-U_{\tau} \sum_{i}\left(1-2 n_{i_{+}}\right)\left(1-2 n_{(i+1)_{-}}\right)$and take the $U_{\tau} \rightarrow+\infty$ limit. In this case, the interaction locks $n_{i_{+}}=n_{(i+1)_{-}}$. In the low-energy subspace, we introduce

$$
\begin{aligned}
& E_{i+\frac{1}{2}}=1-2 n_{i_{+}}=1-2 n_{(i+1)_{-}}, \\
& V_{i+\frac{1}{2}}=\lambda_{i_{+}}(-1)^{n_{i_{+}}} \lambda_{(i+1)_{-}} .
\end{aligned}
$$

After the bosonization on the boundary, the above becomes

$$
E_{i+\frac{1}{2}}=\tau_{i_{+}}^{z}=\tau_{(i+1)_{-}}^{z}, \quad V_{i+\frac{1}{2}}=\tau_{i_{+}}^{x} \tau_{(i+1)_{-}}^{x},
$$


which satisfies

$$
E_{i+\frac{1}{2}} V_{i+\frac{1}{2}}=-V_{i+\frac{1}{2}} E_{i+\frac{1}{2}} \text {. }
$$

Now, the $Z_{2}^{K}$-gauge constraint becomes

$$
-E_{i-\frac{1}{2}} \sigma_{i_{+}}^{z} \sigma_{i_{-}}^{z} E_{i+\frac{1}{2}}=1 .
$$

The effective $Z_{2}^{G}$-symmetry generator becomes

$$
\hat{U}_{Z_{2}}=\prod_{i} \sigma_{i_{+}}^{x} \sigma_{(i+1)_{-}}^{x} \mathrm{i}^{\left(1-\sigma_{i_{+}}^{z} \sigma_{(i+1)_{-}}^{z}\right) / 2} .
$$

We can write down a $Z_{2}^{G}$-symmetric and local $Z_{2}^{K}$-gaugesymmetric boundary effective Hamiltonian:

$$
\begin{aligned}
H= & -\sum_{i} V_{i+\frac{1}{2}}(|\uparrow \uparrow\rangle\langle\downarrow \downarrow|+| \downarrow \downarrow\rangle\langle\uparrow \uparrow|)_{i_{+},(i+1)_{-}} \\
& -J \sum_{i} \sigma_{i_{+}}^{z} \sigma_{(i+1)_{-}}^{z}-U \sum_{i} E_{i+\frac{1}{2}} \\
= & -\sum_{i} V_{i+\frac{1}{2}}\left(\sigma_{i_{+}}^{+} \sigma_{(i+1)_{-}}^{+}+\sigma_{i_{+}}^{-} \sigma_{(i+1)_{-}}^{-}\right) \\
& -J \sum_{i} \sigma_{i_{+}}^{z} \sigma_{(i+1)_{-}}^{z}-U \sum_{i} E_{i+\frac{1}{2}},
\end{aligned}
$$

which is identical to the effective boundary Hamiltonian in Eq. (A23) in Appendix A 2.

Note that all the low-energy excitations at an energy scale much less than $U_{\tau}$ are purely bosonic. So, the fermionic CZX model has a boundary that can be identified as a boundary of the bosonic CZX model, stacking with a fermionic product state. This implies that the ground state of the fermionic CZX model can also be viewed as a bosonic $Z_{2}^{G}$-SPT state, stacking with fermionic product states.

\section{APPENDIX D: SYMMETRY-EXTENDED GAPPED BOUNDARIES OR INTERFACES: COMMENTS, CRITERIA, AND EXAMPLES}

In this section, we aim to show many systematic examples of $G$-topological states, such that we can construct an $H$-gapped boundary or interface through the symmetry extension mechanism, based on a group homomorphism $r$ (a surjective epimorphism) by a short exact sequence,

$$
1 \rightarrow K \rightarrow H \stackrel{r}{\rightarrow} G \rightarrow 1 .
$$

In Sec. IV D 1, we considered the mathematical setup in which the $G$-cocycle is trivialized in $H$ based on homogeneous cocycles $\nu_{d}^{G}$, in order to consider SPT states. In this Appendix D, instead, we set up the mathematics based on inhomogeneous cocycles $\omega_{d}^{G}$, for the convenience of notations (which becomes more transparent later) and for more general topological phases (SET states and intrinsic topological orders).

The plan of this Appendix D is the following. In Appendixes D 1 and D 2, we will give an overview of the setup of problems on the boundaries or interfaces. In Appendix (A3), we show that the Lyndon-HochschildSerre (LHS) spectral sequence criteria are helpful to analytically derive some split $H$-cochains that can trivialize certain $G$-cochains (that can be $G$-cocycles) of one higher dimension. The advantage of this LHS approach, compared to Sec. V, is that we can obtain some analytic split $\mathrm{H}$ cochains [91]. However, the drawback of this LHS approach is that, in a few cases, the $G$-cochains may not always be the $G$-cocycles that we hoped for (standing for nontrivial $G$-topological phases) but $G$-coboundaries (standing for a trivial vacuum). Nevertheless, we can still produce many valid successful examples through Appendix (A3)'s LHS approach shown later in Appendix D. For all the examples given from Appendixes D 4 to D 23, all that we aim to provide are the data of the inhomogeneous $G$-cocycle $\omega_{d}^{G}(g)$ and its trivialization by finding the split $H$-cochain $\beta_{d-1}^{H}(h)$.

\section{Symmetry extension setup: Trivialize a $G$-cocycle to an $\boldsymbol{H}$-coboundary (split to lower-dimensional $H$-cochains) by lifting $G$ to a larger group $H$}

We switch to using the inhomogeneous version of $d$-cocycles $\omega_{d}$ and $d$-cochains $\beta_{d}$ for the convenience of notations. The inhomogeneous version is more general and suitable even for gauge theories with nontrivial holonomies around noncontractible cycles. Moreover, we can convert between $\nu_{d}^{G}$ and $\omega_{d}^{G}$ based on the well-known relation given in Eq. (9.6). We can develop their path integrals, lattice Hamiltonians, and wave functions suitable for many-body quantum systems as in Sec. IX.

The setup of the symmetry extension in Eq. (D1) for inhomogeneous cocycles goes as follows. By pulling back a $G$-cocycle $\omega_{d}^{G}$ back to $H$, it becomes an $H$-coboundary $\delta \beta_{d-1}^{H}$. Formally, we mean that a nontrivial $G$-cocycle

$$
\omega_{d}^{G}(g) \in \mathcal{H}^{d}[G, U(1)]
$$

becomes a trivial element 1 (a coboundary) when it is pulled back (denoted as ${ }^{*}$ ) to $H$ :

$$
r^{*} \omega_{d}^{G}(g)=\omega_{d}^{G}[r(h)]=\omega_{d}^{H}(h)=\delta \beta_{d-1}^{H}(h) \in \mathcal{H}^{d}[H, U(1)] .
$$

This trivial element means a trivial group element 0 in the cohomology group $\mathcal{H}^{d}[H, U(1)]$ or a coboundary 1 for the $U(1)$ coefficient. The above variable $g$ (or $h$ ) in the bracket is a shorthand of many copies of group elements in a direct product group of $G$ (or $H$ ). More precisely, we rewrite the above in terms of splitting a inhomogeneous $G$-cocycle: 


$$
\begin{aligned}
\omega_{d}^{G}\left(g_{01}, \ldots, g_{d-1 d}\right)= & \omega_{d}^{G}\left[r\left(h_{01}\right), \ldots, r\left(h_{d-1 d}\right)\right]=\omega_{d}^{H}\left(h_{01}, \ldots, h_{d-1 d}\right) \\
= & \left(\beta_{d-1}^{H}\right)^{s\left(h_{01}\right)}\left(h_{12}, \ldots, h_{i-1 i}, h_{i i+1}, h_{i+1 i+2}, \ldots, \cdots, h_{d-1 d}\right) \\
& \times \prod_{i=0}^{d-2} \beta_{d-1}^{H(-1)^{i+1}}\left(h_{01}, \ldots, h_{i-1 i}, h_{i i+1} h_{i+1 i+2}, h_{i+2 i+3}, \ldots, \cdots, h_{d-1 d}\right) \\
& \times \beta_{d-1}^{H(-1)^{d}}\left(h_{01}, \ldots, h_{i-1 i}, h_{i i+1}, h_{i+1 i+2}, \ldots, \cdots, h_{d-2 d-1}\right) \\
\equiv & \delta \beta_{d-1}^{H} .
\end{aligned}
$$

Because of the property of the $G$-module for the cohomology group of $U(1)$ cocycles, we impose that $\left(\beta_{d-1}^{H}\right)^{s(h)}=$ $\beta_{d-1}^{H}$ for $h$ contains only a unitary group element, and $\left(\beta_{d-1}^{H}\right)^{s(h)}=\left(\beta_{d-1}^{H}\right)^{-1}$ for $h$ is an antiunitary group element in $H$, such as an antiunitary time-reversal symmetry group.

We call this approach "symmetry extension" (or, colloquially, "symmetry enhancement"), because $H$ is a larger group mapping surjectively to $G$. For quantum many-body systems, the dimension of Hilbert space is enhanced from a $|G|$ per degree of freedom in the bulk to a larger $|H|$ per degree of freedom on the boundary.

Here, we provide some useful information of the cohomology group $\mathcal{H}^{d}[G, U(1)]$ of $G$ that may be used later:

We write the order- 8 dihedral group as

$$
D_{4}=\left\langle x, R \mid R^{4}=x^{2}=1, x R x=R^{-1}\right\rangle,
$$

generated by $x$ and $R$. We write the order- 8 quaternion as

$$
Q_{8}=\left\langle x, y \mid x^{2}=y^{2}, x y x^{-1}=y^{-1}, x^{4}=y^{4}=1\right\rangle,
$$

so that each element in $Q_{8}$ we can write uniquely as $x^{q} y^{n}$, where $q \in\{0,1\}$ and $n \in\{0,1,2,3\}$. For $(q, n) \in$ $\{(0,0),(0,1),(0,2),(0,3),(1,0),(1,1),(1,2),(1,3)\}$, we can identify them as the well-known $Q_{8}$ notation as $x^{q} y^{n} \in\{1, i,-1,-i, j,-k,-j, k\}$.

For notation convention, we use the additive notation 0 to denote the trivial group if all groups are finite Abelian groups such as in $0 \rightarrow Z_{2}^{K} \rightarrow Z_{4}^{H} \rightarrow Z_{2}^{G} \rightarrow 0$. We use the multiplicative notation 1 to denote the trivial group if some group is non-Abelian such as in $1 \rightarrow Z_{4}^{K} \rightarrow Q_{8}^{H} \rightarrow$ $Z_{2}^{G} \rightarrow 1$.

For some selected examples below (from Appendix D 4 to D 23), we will test the LHS spectral sequence $d_{2}$ map technique in Appendix D 3 and comment on its validity to derive $H$-cochains for trivializing certain $G$-cocycles.

\section{Symmetry-extended gapped interfaces}

Consider the interface (i.e., domain wall) between two sides of phases labeled by groups $G_{\mathrm{I}}$ and $G_{\mathrm{II}}$, respectively. The two sides of phases could be both SPTs, both SETs, or both topological orders. Below, we present various systematic constructions for gapped interfaces. The gapped boundary of $G$ can be regarded as a gapped interface between a $G$-topological state and a trivial vacuum.

\section{a. Symmetry extension and the folding trick: Trivialize a $G_{I} \times G_{I I}$-cocycle to an $H$-coboundary by splitting to lower-dimensional $\boldsymbol{H}$-cochains}

Importantly, the previous formulation of a gapped boundary is also applicable to formulate the gapped interface, by using the folding trick. The strategy is that, by replacing the $G$ in Appendix D 1 with $G_{\mathrm{I}} \times G_{\mathrm{II}}$, we can determine the gapped boundary between $G_{\mathrm{I}} \times G_{\mathrm{II}}$ and the vacuum, via trivializing a $G_{\mathrm{I}} \times G_{\mathrm{II}}$-cocycle to an $H$ coboundary by splitting to lower-dimensional $H$-cochains. The surjective group homomorphism $r$ is given by

$$
1 \rightarrow K \rightarrow H \stackrel{r}{\rightarrow} G_{\mathrm{I}} \times G_{\mathrm{II}} \rightarrow 1 .
$$

We can rewrite the above in terms of splitting an inhomogeneous $G=G_{\mathrm{I}} \times G_{\mathrm{II}}$-cocycle:

$$
\omega_{d}^{G_{\mathrm{I}} \times G_{\mathrm{II}}}(g)=\omega_{d}^{G_{\mathrm{I}} \times G_{\mathrm{II}}}[r(h)]=\delta \beta_{d-1}^{H}(h) .
$$

Here, $(g)$ is a shorthand of $\left(g_{01}, \ldots, g_{d-1 d}\right)$ with each element in $G_{\mathrm{I}} \times G_{\mathrm{II}}$. Generally, $\omega^{G_{\mathrm{I}} \times G_{\mathrm{II}}}$ is a cocycle in the cohomology group $\mathcal{H}^{d}\left[G_{\mathrm{I}} \times G_{\mathrm{II}}, U(1)\right]$. The Künneth theorem shows us that there exists a particular form of cocycle $\omega_{\mathrm{I}}^{G_{\mathrm{I}}}\left(g_{\mathrm{I}}\right) \cdot \omega_{\mathrm{II}}^{G_{\mathrm{II}}}\left(g_{\mathrm{II}}\right)^{-1}$, obtained from $\omega_{\mathrm{I}}^{G_{\mathrm{I}}} \in$ $\mathcal{H}^{d}\left[G_{\mathrm{I}}, U(1)\right]$ and $\omega_{\text {II }}^{G_{\text {II }}} \in \mathcal{H}^{d}\left[G_{\mathrm{II}}, U(1)\right]$. Now, we see that the $G_{\mathrm{I}^{-}}$-symmetry action only acts on $\omega_{\mathrm{I}}^{G_{\mathrm{I}}}\left(g_{\mathrm{I}}\right)$, while the $G_{\mathrm{II}^{-}}$ symmetry action only acts on $\omega_{\text {II }}^{G_{\mathrm{II}}}\left(g_{\mathrm{II}}\right)$. By folding $\omega_{\mathrm{I}}^{G_{\mathrm{I}}}\left(g_{\mathrm{I}}\right)$ and $\omega_{\text {II }}^{G_{\text {II }}}\left(g_{\text {II }}\right)$ to two different sides of the $H$-gapped boundary, we obtain an $\mathrm{H}$-gapped interface.

\section{b. Append a lower-dimensional topological state onto the boundary or interface}

For all the previous setups, we actually pick a trivialization of the pullback of the $G$-cocycle to $H$. The possible trivialization choices differed by a class in $\mathcal{H}^{d-1}[H, U(1)]$ physically imply that we can further append lowerdimensional gapped topological states (that are well defined in their own dimension) onto the boundary or the interface. (See also Sec. VIII B for a discussion.) In general, it could be 
a SET of $(d-1)$ dimensions labeled by an $H$-cocycle with $H$ site and $K$ link variables:

$$
\begin{aligned}
& \mathcal{V}_{d-1}^{H, K}\left(\left\{h_{i}\right\} ;\left\{k_{i j}\right\}\right) \\
& \quad=\nu_{d-1}^{H}\left(h_{i_{0}}, k_{i_{0} i_{1}} h_{i_{1}}, \ldots, k_{i_{0} i_{1}} \ldots k_{i_{d-2} i_{d-1}} h_{i_{d-1}}\right) \\
& \quad \in \mathcal{H}^{d-1}[H, U(1)]
\end{aligned}
$$

and described by $1 \rightarrow K \rightarrow H \rightarrow G \rightarrow 1$, with a total projective symmetry group $H$, a gauge group $K$, and a global symmetry group $G$. The $H$-cocycle obeys the cocycle condition: $\delta \mathcal{V}_{d-1}^{H, K}=\delta \nu_{d-1}^{H}=1$. In different limit choices of $G$ and $K$, the topological phases of $\mathcal{V}_{d-1}^{H, K}$ include SPTs, topological orders and SETs.

The proper choices of $G$ and $K$ on the boundary are also constrained by the choices of $G$ and $K$ in the bulk. We will leave this issue as a case-by-case study.

In this Appendix D, we use inhomogeneous cocycles as, in Appendix D 1, we replace $\mathcal{V}_{d-1}^{H, K}$ by $\Omega_{d-1}^{H}$. We see that

$$
\begin{aligned}
\delta\left[\beta_{d-1}^{H}(h) \Omega_{d-1}^{H}(h)\right] & =\delta\left[\beta_{d-1}^{H}(h)\right]=\omega_{d}^{H}(h) \\
& =\omega_{d}^{G}[r(h)]=\omega_{d}^{G}(g),
\end{aligned}
$$

where $\delta\left[\Omega_{d-1}^{H}(h)\right]=1$. It can also be appended on the interface, as in Appendix D 2 a's Eq. (D5),

$$
\begin{aligned}
\delta\left[\beta_{d-1}^{H}(h) \Omega_{d-1}^{H}(h)\right] & =\delta \beta_{d-1}^{H}(h) \\
& =\omega_{d}^{G_{\mathrm{I}} \times G_{\mathrm{II}}}[r(h)]=\omega_{d}^{G_{\mathrm{I}} \times G_{\mathrm{II}}}(g) .
\end{aligned}
$$

Here, the appended lower-dimensional topological states [differed by $\Omega_{d-1}^{H}$, with $\left.\delta\left[\Omega_{d-1}^{H}(h)\right]=1\right]$ are all gapped.

\section{Criteria on trivializing the $G$-cocycle in a larger group $H$ : Lyndon-Hochschild-Serre spectral sequence}

We would like to provide a systematic way to determine the possible trivialization of the $d$-cocycle in $G$ by lifting to a larger group $H$, based on the setup of the LHS spectral sequence. The question we would like to address here is, "Given $1 \rightarrow K \rightarrow H \stackrel{r}{\rightarrow} G \rightarrow 1$, how can we analytically obtain the split $H$-cochain $\beta_{d-1}^{H}$ that satisfies that $\omega_{d}^{G}=$ $\delta \beta_{d-1}^{H}$ for some $G$-cocycle $\omega_{d}^{G}$ ?"

The answer is as follows. For $1 \rightarrow K \rightarrow H \stackrel{r}{\rightarrow} G \rightarrow 1$, with $G$ acting trivially on $\mathcal{H}^{*}[K, U(1)]$ [92], there is a spectral sequence $\left\{E_{n}^{p, q}, d_{n}\right\}$ with

(a) $E_{2}^{p, q}=\mathcal{H}^{p}\left(G, \mathcal{H}^{q}[K, U(1)]\right)$.

(b) The differential is defined as a map $d_{n}: E_{n}^{p, q} \rightarrow$ $E_{n}^{p+n, q-n+1}$. We have $E_{n+1}^{p, q}=\left[\operatorname{Ker}\left(d_{n}\right) / \operatorname{Im}\left(d_{n}\right)\right]$ at $E_{n}^{p, q}$.

We focus on the $d_{2}$ differential of the $E_{2}$ page in the LHS spectral sequence,

$$
d_{2}: E_{2}^{p, q} \rightarrow E_{2}^{p+2, q-1}
$$

$\Rightarrow d_{2}: \mathcal{H}^{p}\left(G, \mathcal{H}^{q}[K, U(1)]\right) \rightarrow \mathcal{H}^{p+2}\left(G, \mathcal{H}^{q-1}[K, U(1)]\right)$,

in particular,

$$
\begin{aligned}
d_{2} & : \mathcal{H}^{d-2}\left(G, \mathcal{H}^{1}[K, U(1)]\right) \\
& \rightarrow \mathcal{H}^{d}\left(G, \mathcal{H}^{0}[K, U(1)]\right)=\mathcal{H}^{d}[G, U(1)] .
\end{aligned}
$$

If we want to trivialize the $d$-cocycle $\omega_{d}^{G} \in \mathcal{H}^{d}[G, U(1)]$, we can look for a larger group $H$, where $H / K=G$ for some $K$. The $d_{2}$ turns out to provide the following nice property. The image of the differential $d_{2}: \mathcal{H}^{d-2}\left(G, \mathcal{H}^{1}[K, U(1)]\right) \rightarrow$ $\mathcal{H}^{d}[G, U(1)]$ provides elements of $\omega_{d}^{G} \in \mathcal{H}^{d}[G, U(1)]$, such that all such elements are guaranteed to vanish to be trivial as a coboundary in $\mathcal{H}^{d}[H, U(1)]$. In other words, every element $\omega_{d}^{G}$ in the image of the $d_{2}$ map is guaranteed to be trivial in $\mathcal{H}^{d}[H, U(1)]$. [93] We have

$$
\omega_{d}^{G}=\delta \beta_{d-1}^{H}
$$

or, more precisely,

$$
\omega_{d}^{G}[r(h)]=\omega_{d}^{H}(h)=\delta \beta_{d-1}^{H}(h),
$$

where $\beta_{d-1}^{H}$ is determined by the $d_{2}$ differential and the map

$$
f: G^{d-2} \rightarrow \mathcal{H}^{1}[K, U(1)] .
$$

The $f$ is a function that relates to a cocycle

$$
\alpha_{d-2} \in \mathcal{H}^{d-2}\left(G, \mathcal{H}^{1}[K, U(1)]\right) .
$$

If we know the data of $H$ are given by the pair $G$ and $K$, we can write $\beta_{d-1}^{H}$ as a function of $d_{2}\left(\alpha_{d-2}\right)$. Notice that $d_{2}\left(\alpha_{d-2}\right)$ is in $\mathcal{H}^{d}[G, U(1)]$. The claim is that there exists a map $d_{2}: \mathcal{H}^{d-2}\left(G, \mathcal{H}^{1}[K, U(1)]\right) \rightarrow \mathcal{H}^{d}[G, U(1)]$, where every $G$-cocycle $\omega_{d}$ in the image of the $d_{2}$ map is an $H$-coboundary that can be split to lower-dimensional $H$-cochains.

By writing the group element $h \in H$ in terms of a pair $(k, g) \in(K, G)$ as $h=(k, g)$, we can write down the further precise relation,

$$
\begin{aligned}
\omega_{d}^{H}(h) & =\omega_{d}^{H}\left(h_{1}, h_{2}, \ldots, h_{d}\right) \\
& =\omega_{d}^{H}\left[\left(k_{1}, g_{1}\right),\left(k_{2}, g_{2}\right), \ldots,\left(k_{d}, g_{d}\right)\right] \\
& =\omega_{d}^{G}\left(g_{1}, g_{2}, \ldots, g_{d}\right)=\omega_{d}^{G}(g) \\
& =\delta\left(\beta_{d-1}^{H}\left[\left(k_{1}, g_{1}\right),\left(k_{2}, g_{2}\right), \ldots,\left(k_{d-1}, g_{d-1}\right)\right]\right) \\
& =\left(\delta \beta_{d-1}^{H}\right)\left[\left(k_{1}, g_{1}\right),\left(k_{2}, g_{2}\right), \ldots,\left(k_{d}, g_{d}\right)\right] \\
& =\left(\delta \beta_{d-1}^{H}\right)\left(h_{1}, h_{2}, \ldots, h_{d}\right)=\delta \beta_{d-1}^{H}(h) .
\end{aligned}
$$

Such a construction of $\beta_{d-1}^{H}$ as a function of $d_{2}\left(\alpha_{d-2}\right)$ from the LHS spectral sequence can derive some $G$-coycle $\omega_{d}^{G}[r(h)]=\omega_{d}^{H}(h)=\delta \beta_{d-1}^{H}(h)$ that can split to lowerdimensional $H$-cochains. However, we would like to emphasize that some obtained $\omega_{d}^{G}[r(h)]$ may be already 
a $G$-coboundary and may not be the specific nontrivial $G$-cocycle that we originally aimed to trivialize. We will show in Appendix D (from Appendixes D 4 to D 23) how this LHS spectral sequence approach can help in constructing some examples, but not necessarily other examples.

\section{4. $2+1 / 1+1 \mathrm{D}$ bosonic $0 \rightarrow Z_{2}^{K} \rightarrow Z_{4}^{H} \rightarrow Z_{2}^{G} \rightarrow 0$}

Consider the example where $G=Z_{2}, H=Z_{4}$, and $K=Z_{2}$, and denote them under $0 \rightarrow Z_{2}^{K} \rightarrow Z_{4}^{H} \rightarrow Z_{2}^{G} \rightarrow 0$. The twisted three-cocycle is

$$
\begin{aligned}
\omega_{3}^{Z_{2}^{G}}\left(g_{a}, g_{b}, g_{c}\right)= & \exp \left[\frac { \mathrm { i } 2 \pi } { 2 ^ { 2 } } p [ g _ { a } ] _ { 2 } \left(\left[g_{b}\right]_{2}+\left[g_{c}\right]_{2}\right.\right. \\
& \left.\left.-\left[\left[g_{b}\right]_{2}+\left[g_{c}\right]_{2}\right]\right)\right]=(-1)^{g_{a} g_{b} g_{c}}
\end{aligned}
$$

with $g \in Z_{2}^{G}$ and $p \in \mathcal{H}^{3}\left[Z_{2}^{G}, U(1)\right]=\mathbb{Z}_{2}$. To have a nontrivial three-cocycle, we set $p=1$. This cocycle is equivalent to $\mathrm{e}^{\mathrm{i} 2 \pi \int \frac{1}{2} a_{1} \cup a_{1} \cup a_{1}}=(-1)^{\int a_{1} \cup a_{1} \cup a_{1}}$ with a cup product form of $a_{1} \cup a_{1} \cup a_{1}$, in $\mathcal{H}^{3}\left[Z_{2}, U(1)\right]$. The $a_{1}$ here is a $\mathbb{Z}_{2}$-valued one-cocycle in $\mathcal{H}^{1}\left(M^{3}, \mathbb{Z}_{2}\right)$ on the spacetime complex $M^{3}$. For a discrete finite $G$, the principle $G$-bundle and the flat $G$ connection are effectively the same. Here, we consider $G=Z_{2}$, so, in this context, we can view the nontrivial SPTs detectable by the principle $Z_{2}$-bundle and the flat $Z_{2}$-connection. The boundary bosonic anomaly of SPTs is explored in Ref. [96].

We find that the analytic two-cochain,

$$
\beta_{2}\left(h_{1}, h_{2}\right)=\exp \left[(\mathrm{i} 2 \pi p / 4)\left[h_{1}\right]_{2}\left[h_{2}\right]_{4}\right],
$$

splits $G$ three-cocycle. Alternatively, we can choose $\beta_{2}\left(h_{1}, h_{2}\right)=\exp \left[(\mathrm{i} 2 \pi p / 4)\left[h_{1}\right]_{4}\left[h_{2}\right]_{2}\right]$ with $m, n \in Z_{4}^{H}$.

Furthermore, we find that the LHS technique in Appendix D 3 works successfully. For the LHS technique of Appendix D 3, we look for

$$
\begin{gathered}
d_{2}: \mathcal{H}^{1}\left(G, \mathcal{H}^{1}[K, U(1)]\right) \rightarrow \mathcal{H}^{3}\left(G, \mathcal{H}^{0}[K, U(1)]\right) \\
=\mathcal{H}^{3}[G, U(1)], \\
\Rightarrow d_{2}: \mathcal{H}^{1}\left(Z_{2}, Z_{2}\right)=\mathbb{Z}_{1} \rightarrow \mathcal{H}^{3}\left[Z_{2}, U(1)\right]=\mathbb{Z}_{2}, \\
f: G \rightarrow \mathcal{H}^{1}[K, U(1)] \\
\Rightarrow f: Z_{2}^{G} \rightarrow \mathcal{H}^{1}\left[Z_{2}^{K}, U(1)\right]=\mathbb{Z}_{2} .
\end{gathered}
$$

Because this $f$ maps to $\mathcal{H}^{1}\left[Z_{2}^{K}, U(1)\right]=\mathbb{Z}_{2}$, the $\beta_{2}$ can be a base of $(-1)$. We find that another two-cochain that splits the three-cocycle is

$$
\tilde{\beta}_{2}\left(h_{1}, h_{2}\right)=f\left(g_{2}\right)^{k_{1}}=(-1)^{g_{2} k_{1}} .
$$

For $h=0,(g, k)=(0,0)$; for $h=1,(g, k)=(1,0)$; for $h=2,(g, k)=(0,1)$; and for $h=3,(g, k)=(1,1)$. The group elements in $H$ satisfy

$$
\begin{aligned}
h_{1} \cdot h_{2} & =\left(g_{1}, k_{1}\right) \cdot\left(g_{2}, k_{2}\right) \\
& =\left(\left[g_{1}+g_{2}\right]_{2},\left[k_{1}+k_{2}+g_{1} g_{2}\right]_{2}\right) .
\end{aligned}
$$

We would like to check that $\left(\delta \tilde{\beta}_{2}\right)\left(h_{1}, h_{2}, h_{3}\right)=(-1)^{g_{1} g_{2} g_{3}}$ :

$$
\begin{aligned}
\left(\delta \tilde{\beta}_{2}\right)\left(h_{1}, h_{2}, h_{3}\right) & =\frac{\tilde{\beta}_{2}\left(h_{2}, h_{3}\right) \tilde{\beta}_{2}\left(h_{1}, h_{2} h_{3}\right)}{\tilde{\beta}_{2}\left(h_{1} h_{2}, h_{3}\right) \tilde{\beta}_{2}\left(h_{1}, h_{2}\right)} \\
& =\frac{(-1)^{g_{3} k_{2}}(-1)^{\left[g_{2}+g_{3}\right]_{2} k_{1}}}{(-1)^{g_{3}\left[k_{1}+k_{2}+g_{1} g_{2}\right]_{2}}(-1)^{g_{2} k_{1}}} \\
= & \frac{(-1)^{g_{3} k_{2}}(-1)^{\left(g_{2}+g_{3}\right) k_{1}}}{(-1)^{g_{3}\left(k_{1}+k_{2}+g_{1} g_{2}\right)}(-1)^{g_{2} k_{1}}}=(-1)^{g_{1} g_{2} g_{3}},
\end{aligned}
$$

which is true. [Actually, both $\tilde{\beta}_{2}\left(h_{1}, h_{2}\right)=(-1)^{g_{2} k_{1}}$ and $\tilde{\beta}_{2}\left(h_{1}, h_{2}\right)=(-1)^{g_{1} k_{2}}$ work to trivialize the $G$ threecocycle.] We can rewrite $\tilde{\beta}_{2}\left(h_{1}, h_{2}\right)=(-1)^{g_{2} k_{1}}=$ $(-1)^{g_{2}\left(h_{1}-\left[h_{1}\right] / 2\right)}=i^{g_{2}\left(h_{1}-\left[h_{1}\right]_{2}\right)}=i^{\left[h_{2}\right]_{2}\left(\left[h_{1}\right]_{4}-\left[h_{1}\right]_{2}\right)}$. If $\quad$ we write $h \in H$ in terms of $h=(g, k)$, then $\beta_{2}\left(h_{1}, h_{2}\right)=$ $\exp \left[(2 \pi \mathrm{i} / 4)\left(\left[h_{1}\right]_{2}\right)\left(\left[h_{2}\right]_{4}\right)\right]=i^{\left[h_{1}\right]_{2}\left[h_{2}\right]_{4}}=i^{\left[g_{1}\right]_{2}\left[g_{2}+2 k_{2}\right]_{4}}$.

If we consider the bulk to be a fully gauged, topologically ordered state, this becomes a gapped boundary for a bulk $2+1 \mathrm{D}$ field theory of an action $\int(2 / 2 \pi) B d A+$ $(1 / 2 \pi) A d A$, with $B$ and $A$ locally as one-form gauge fields.

\section{a. Degeneracy on a disk and an annulus: Partition functions $Z\left(D^{2} \times S^{1}\right)$ and $Z\left(I^{1} \times S^{1} \times S^{1}\right)$}

Here, we can put the $2+1 / 1+1 \mathrm{D} 0 \rightarrow Z_{2}^{K} \rightarrow Z_{4}^{H} \rightarrow$ $Z_{2}^{G} \rightarrow 0$ construction of topological states on a spatial $D^{2}$ disk or an annulus $I^{1} \times S^{1}$ to count the degeneracy (GSD). Whether we gauge the global symmetry $K$ and $H$ or not, we have at least three types of theories:

(i) Fully global symmetric SPTs (a bulk $G$-SPT and a boundary anomalous $H$-SPT),

(ii) Bulk SPTs or boundary SETs (a bulk $G$-SPT and a boundary anomalous $H$-SET with a gauge group $K$ ),

(iii) Fully topological orders with dynamical gauge fields (a bulk $G$-topologically ordered gauge theory and a boundary anomalous $H$-gauge theory). Since $K$ is a normal subgroup in $H$, we can label the $K$-holonomy in $H$. Thus, below, we write all holonomies $h$ in $H$.

Theory (i) is basically the second boundary discussed in Secs. III and IV. Theory (ii) is basically the third (hardgauge) and fourth (soft-gauge) boundaries discussed in Secs. III and IV. Theory (iii) is basically the fully dynamical gauge boundary without global symmetry.

We compute the partition function of Sec. IX A 5 on $Z\left(D^{2} \times S^{1}\right)$ to evaluate GSD on a spatial $D^{2}$ disk in Table I.

Note that the $h=0$ carries zero or an even $Z_{2}^{G}$ charge. The $h=2$ carries an odd $Z_{2}^{G}$ charge. For theory (iii), when the $Z_{2}^{G}$ is gauged, the ground state for the whole system cannot carry an odd $Z_{2}^{G}$ charge; thus, $h=0 \in H$ implies $\mathrm{GSD}=1$ on a disk. An important remark is that we cannot 
TABLE I. For theory (ii), GSD $=2$ from the holonomy $h=0$ and $h=2 \in H$. For the fully gauge theory (iii), GSD $=1$ from the holonomy $h=0 \in H$.

\begin{tabular}{lccc}
\hline \hline Disk & $\begin{array}{c}\text { Theory (i) } \\
\text { (second boundary) }\end{array}$ & $\begin{array}{c}\text { Theory (ii) (third or } \\
\text { fourth boundary) }\end{array}$ & $\begin{array}{c}\text { Theory (iii) } \\
\text { (fifth boundary) }\end{array}$ \\
\hline$D^{2}$ & 1 & 2 & 1 \\
\hline \hline
\end{tabular}

regard the $1+1 \mathrm{D}$ anomalous $Z_{4}^{H}$ gauge theory as a usual $1+1 \mathrm{D}$ discrete gauge theory, because the usual $1+1 \mathrm{D} Z_{4}$ gauge theory has GSD $=|H|=4$ on an $S^{1}$ ring. In our case, the $2+1 \mathrm{D}$ bulk plays an important rule, which causes the GSD to decrease to GSD $=1$ for theory (iii).

We compute the partition function of Sec. IX A 5 on $Z\left(I^{1} \times S^{1} \times S^{1}\right)$ to evaluate GSD on an annulus $I^{1} \times S^{1}$ in Table II.

Again, the $2+1 \mathrm{D}$ bulk plays an important rule for the GSD reduction for theory (iii) from GSD $=|H|^{2}=16$ to GSD $=2$ in Table II.

\section{5. $d+1 / d \mathrm{D}$ bosonic $0 \rightarrow Z_{2}^{K} \rightarrow Z_{4}^{H} \rightarrow Z_{2}^{G} \rightarrow 0$ for an even $d$}

We can readily generalize Appendix D 4 to consider a gapped boundary for the $d+1 / d \mathrm{D}$ bosonic SPTs with a $G=Z_{2}$ symmetry for any even dimension $d$ under $0 \rightarrow Z_{2}^{K} \rightarrow Z_{4}^{H} \rightarrow Z_{2}^{G} \rightarrow 0$. The twisted $(d+1)$-cocycle is

$$
\omega_{d+1}^{Z_{2}^{G}}\left(g_{1}, g_{2}, \ldots, g_{d+1}\right)=(-1)^{g_{1} g_{2} \ldots g_{d+1}},
$$

TABLE II. For theory (ii), GSD $=4$ from the holonomies: $\left(h_{\text {in }}, h_{\text {out }}\right)$ with $h_{\text {in }}, h_{\text {out }} \in\{0,2\}$. For the fully gauge theory (iii), $\mathrm{GSD}=2$ from the holonomies of two sectors: $\left(h_{\text {in }}, h_{\text {out }}\right)=$ $(0,0),(2,2)$.

Annulus Theory (i) Theory (ii) (third or Theory (iii) $S^{1} \times I^{1} \quad$ (second boundary) fourth boundary) (fifth boundary)

\begin{tabular}{llll}
\hline GSD & 1 & 4 & 2 \\
\hline \hline
\end{tabular}

with $g \in Z_{2}^{G}$ and $\mathcal{H}^{d+1}\left[Z_{2}^{G}, U(1)\right]=\mathbb{Z}_{2}$ for an even $d$. This cocycle is equivalent to $\mathrm{e}^{\mathrm{i} 2 \pi \int \frac{1}{2} a_{1} \cup a_{1} \cup \ldots \cup a_{1}}$ with a cup product form of $a_{1} \cup a_{1} \cup \ldots \cup a_{1}$, in $\mathcal{H}^{d+1}\left[Z_{2}, U(1)\right]$. The $a_{1}$ here is a $\mathbb{Z}_{2}$-valued one-cocycle in $H^{1}\left(M^{d+1}, \mathbb{Z}_{2}\right)$ on the spacetime complex $M^{d+1}$.

As in Appendix D 4, we write $h=(g, k) \in Z_{4}^{H}$ as a doublet where $g \in Z_{2}^{G}$ and $k \in Z_{2}^{K}$. We find that the $d$-cochain that splits the $(d+1)$-cocycle in $H$ can be

$$
\tilde{\beta}_{d}\left(h_{1}, h_{2}, \ldots, h_{d}\right)=(-1)^{g_{2} \cdots g_{d} k_{1}} .
$$

The group elements in $H$ satisfy

$$
h_{1} \cdot h_{2}=\left(g_{1}, k_{1}\right) \cdot\left(g_{2}, k_{2}\right)=\left(\left[g_{1}+g_{2}\right]_{2},\left[k_{1}+k_{2}+g_{1} g_{2}\right]_{2}\right) \text {. }
$$

We would like to check that $\left(\delta \tilde{\beta}_{d}\right)\left(h_{1}, h_{2}, \ldots, h_{d}, h_{d+1}\right)=$ $(-1)^{g_{1} g_{2} \ldots g_{d+1}}$ for an even $d$. Namely,

$$
\begin{aligned}
\left(\delta \tilde{\beta}_{d}\right)\left(h_{1}, h_{2}, \ldots, h_{d}, h_{d+1}\right) & =\frac{\tilde{\beta}_{d}\left(h_{2}, \ldots, h_{d+1}\right) \ldots \tilde{\beta}_{d}\left(h_{1}, h_{2}, \ldots, h_{d} h_{d+1}\right)}{\tilde{\beta}_{d}\left(h_{1} h_{2}, \ldots, h_{d+1}\right) \ldots \tilde{\beta}_{d}\left(h_{1}, h_{2}, \ldots, h_{d}\right)} \\
& =\frac{(-1)^{g_{3} \cdots g_{d+1} k_{2}}(-1)^{\left(g_{2}+g_{3}\right) g_{4} \cdots g_{d+1} k_{1}} \ldots(-1)^{g_{2} \cdots\left(g_{d}+g_{d+1}\right) k_{1}}}{(-1)^{g_{3} \cdots g_{d+1}\left(k_{1}+k_{2}+g_{1} g_{2}\right)} \ldots(-1)^{g_{2} \cdots g_{d} k_{1}}}
\end{aligned}
$$

is true. Moreover, since $\mathcal{H}^{d}\left[Z_{n}, U(1)\right]=0$ for any even dimension $d$, there is no further lower-dimensional topological phase of the $H=Z_{4}$-cocycle that we can append on the gapped boundary of an even spacetime dimension $d$.

We find that the $d+1 \mathrm{D}$ bosonic SPTs with $Z_{2}$ symmetry (the bosonic topological superconductor of $G=Z_{2}$ ) have a $d \mathrm{D}$ symmetry-preserving surface deconfined $Z_{2}$ topologically ordered gauge theory, at least for $d \geq 4$. When $d=2$, the boundary deconfined $Z_{2}$ gauge theory is a spontaneous symmetry-breaking state crossing over to a confined state; thus, we require fine-tuning to have a deconfined gauge theory, shown in Sec. A 2 d.

If we consider the bulk to be a fully gauged topologically ordered state, this becomes a gapped boundary for a bulk $d+1 \mathrm{D}$ field theory of an action $\int(2 / 2 \pi) B d A+$ $\left[1 /(2 \pi)^{d / 2}\right] A(d A)^{d / 2}=\int(2 / 2 \pi) B d A+\left[1 /(2 \pi)^{d / 2}\right] A d A \cdots d A$, with, locally, $A$ a one-form gauge field and $B$ a $d$-form gauge field.

\section{6. $3+1 / 2+1 \mathrm{D}$ bosonic $0 \rightarrow Z_{2} \rightarrow Z_{4}^{T} \rightarrow Z_{2}^{T} \rightarrow 0$ with $Z_{2}^{T}$ time-reversal symmetry}

We discussed this example in the main text of Sec. V C through a different method. From Ref. [15] and Table III, for an antiunitary symmetry $Z_{2}^{T}$, we recall that the cohomology groups for an odd dimension $d$ offer $\mathcal{H}^{4}\left[Z_{2}^{T}, U_{T}(1)\right]=Z_{2}$. The four-cocycle $\omega_{4}^{Z_{2}^{T}} \in$ $\mathcal{H}^{4}\left[Z_{2}^{T}, U_{T}(1)\right]$ is of a form similar to the cocycle studied in the previous section. The only new ingredient for the calculation involving $Z_{2}^{T}$-symmetry is the nontrivial antiunitary action of $Z_{2}^{T}$ on the $Z_{2}^{T}$-module $U_{T}(1)$. This cocycle is equivalent to $\mathrm{e}^{\mathrm{i} 2 \pi \int \frac{1}{2} w_{1}^{4}}$ in 
TABLE III. Some examples of cohomology group $\mathcal{H}^{d}[G, U(1)]$ for $G=D_{4}, Q_{8}, Z_{2}, Z_{2}^{T}$ and $\left(Z_{2}\right)^{2}$ that can be used to construct $G$-topological phases.

\begin{tabular}{lcccc}
\hline \hline$G$ & $\mathcal{H}^{1}[G, U(1)]$ & $\mathcal{H}^{2}[G, U(1)]$ & $\mathcal{H}^{3}[G, U(1)]$ & $\mathcal{H}^{4}[G, U(1)]$ \\
\hline$D_{4}$ & $\left(\mathbb{Z}_{2}\right)^{2}$ & $\mathbb{Z}_{2}$ & $\left(\mathbb{Z}_{2}\right)^{2} \times \mathbb{Z}_{4}$ & $\left(\mathbb{Z}_{2}\right)^{2}$ \\
$Q_{8}$ & $\left(\mathbb{Z}_{2}\right)^{2}$ & 0 & $\mathbb{Z}_{8}$ & 0 \\
$Z_{2}$ & $\mathbb{Z}_{2}$ & 0 & $\mathbb{Z}_{2}$ & 0 \\
$Z_{2}^{T}$ & 0 & $\mathbb{Z}_{2}$ & 0 & $\mathbb{Z}_{2}$ \\
$\left(Z_{2}\right)^{2}$ & $\left(\mathbb{Z}_{2}\right)^{2}$ & $\mathbb{Z}_{2}$ & $\left(\mathbb{Z}_{2}\right)^{3}$ & $\left(\mathbb{Z}_{2}\right)^{2}$ \\
\hline \hline
\end{tabular}

$\mathcal{H}^{4}\left[Z_{2}^{T}, U_{T}(1)\right]$. The $w_{1}$ here is $\mathbb{Z}_{2}$-valued, the first Stiefel-Whitney class in $H^{1}\left(M^{4}, \mathbb{Z}_{2}\right)$ on the spacetime complex $M^{4} . w_{1} \neq 0$ holds on a nonorientable manifold.

We would like to check that $\omega_{4}^{Z_{2}^{T}}\left(g_{1}, g_{2}, g_{3}, g_{4}\right)=$ $(-1)^{g_{1} g_{2} g_{3} g_{4}}=\left(\delta \tilde{\beta}_{3}\right)\left(h_{1}, h_{2}, h_{3}, h_{4}\right)$ for some $\tilde{\beta}_{3}$. Similar to Appendix D 4, we write $h=(g, k) \in H=Z_{4}^{T}$ as a doublet where $g \in G=Z_{2}^{T}$ and $k \in K=Z_{2}$. We propose $\tilde{\beta}_{3}\left(h_{1}, h_{2}, h_{3}\right)=(-1)^{g_{2} g_{3} k_{1}}$, which splits the $G$-cocycle as an $H$-coboundary under $0 \rightarrow Z_{2} \rightarrow Z_{4}^{T} \rightarrow Z_{2}^{T} \rightarrow 0$. Indeed, we find

$$
\begin{aligned}
& \left(\delta \tilde{\beta}_{3}\right)\left(h_{1}, h_{2}, h_{3}, h_{4}\right) \\
& =\frac{\tilde{\beta}_{3}\left(h_{2}, h_{3}, h_{4}\right) \tilde{\beta}_{3}\left(h_{1}, h_{2} h_{3}, h_{4}\right) \tilde{\beta}_{3}\left(h_{1}, h_{2}, h_{3}\right)}{\tilde{\beta}_{3}\left(h_{1} h_{2}, h_{3}, h_{4}\right) \tilde{\beta}_{3}\left(h_{1}, h_{2}, h_{3} h_{4}\right)} \\
& =\frac{(-1)^{g_{3} g_{4} k_{2}}(-1)^{\left(g_{2}+g_{3}\right) g_{4} k_{1}}(-1)^{g_{2} g_{3} k_{1}}}{(-1)^{g_{3} g_{4}\left(k_{1}+k_{2}+g_{1} g_{2}\right)}(-1)^{g_{2}\left(g_{3}+g_{4}\right) k_{1}}} \\
& =(-1)^{g_{1} g_{2} g_{3} g_{4}},
\end{aligned}
$$

which is true.

We find that the $3+1 \mathrm{D}$ bosonic SPTs with $Z_{2}^{T}$ symmetry (the bosonic topological superconductor of $G=Z_{2}^{T}$ ) have a $2+1 \mathrm{D}$ symmetry-preserving surface deconfined $Z_{2}$ topologically ordered gauge theory.

\section{7. $d+1 / d \mathrm{D}$ bosonic topological superconductor} $0 \rightarrow Z_{2} \rightarrow Z_{4}^{T} \rightarrow Z_{2}^{T} \rightarrow 0$ for an odd $d$ with $Z_{2}^{T}$

time-reversal symmetry: The $d \mathrm{D} Z_{2}^{K}$-gauge theory

boundary of $d+1 \mathrm{D}$ bulk invariant $(-1)^{\int\left(w_{1}\right)^{d+1}}$

From Ref. [15] and Table III, we recall that the cohomology groups for an even dimension $d$ offer

$$
\mathcal{H}^{d+1}\left[Z_{2}, U(1)\right]=Z_{2}, \quad \mathcal{H}^{d+1}\left[Z_{2}^{T}, U_{T}(1)\right]=0 .
$$

The cohomology groups for an odd dimension $d$ offer

$$
\mathcal{H}^{d+1}\left[Z_{2}^{T}, U_{T}(1)\right]=Z_{2}, \quad \mathcal{H}^{d+1}\left[Z_{2}, U(1)\right]=0 .
$$

We can readily generalize Appendix D 6 to consider a gapped boundary for $d+1 / d \mathrm{D}$ bosonic SPTs with a $G=Z_{2}^{T}$ symmetry for any odd dimension $d$ under $0 \rightarrow Z_{2} \rightarrow Z_{4}^{T} \rightarrow Z_{2}^{T} \rightarrow 0$. The twisted $(d+1)$-cocycle is

$$
\omega_{d+1}^{Z_{2}^{G}}\left(g_{1}, g_{2}, \ldots, g_{d+1}\right)=(-1)^{g_{1} g_{2} \ldots g_{d+1}},
$$

with $g \in Z_{2}^{T}$ and $\mathcal{H}^{d+1}\left[Z_{2}^{T}, U_{T}(1)\right]=\mathbb{Z}_{2}$ for an even $d$. This cocycle is equivalent to $\mathrm{e}^{\mathrm{i} 2 \pi \int \frac{1}{2} w_{1}^{d+1}}$ in $\mathcal{H}^{d+1}\left[Z_{2}^{T}, U_{T}(1)\right]$. The $w_{1}$ here is $\mathbb{Z}_{2}$-valued, the first SW class in $H^{1}\left(M^{d+1}, \mathbb{Z}_{2}\right)$ on the spacetime complex $M^{d+1}$. Here, we mean the SW class of the $O(d+1)$ bundle, where $O(d+1)$ is the structure group of the tangent bundle. $w_{1} \neq 0$ holds on a nonorientable manifold.

As in Appendix D 4, we write $h=(g, k) \in H=Z_{4}^{T}$ as a doublet where $g \in G=Z_{2}^{T}$ and $k \in K=Z_{2}$. We find that the $d$-cochain that splits the $(d+1)$-cocycle in $H$ can be

$$
\tilde{\beta}_{d}\left(h_{1}, h_{2}, \ldots, h_{d}\right)=(-1)^{g_{2} \cdots g_{d} k_{1}} .
$$

The group elements in $H$ again satisfy $h_{1} \cdot h_{2}=\left(g_{1}, k_{1}\right) \cdot\left(g_{2}, k_{2}\right)=\left(\left[g_{1}+g_{2}\right]_{2},\left[k_{1}+k_{2}+g_{1} g_{2}\right]_{2}\right)$.

We can check that $\left(\delta \tilde{\beta}_{d}\right)\left(h_{1}, h_{2}, \ldots, h_{d}, h_{d+1}\right)=$ $(-1)^{g_{1} g_{2} \ldots g_{d+1}}$ for an even $d$. Namely,

$$
\begin{aligned}
\left(\delta \tilde{\beta}_{d}\right)\left(h_{1}, h_{2}, \ldots, h_{d}, h_{d+1}\right) & =\frac{\tilde{\beta}_{d}\left(h_{2}, \ldots, h_{d+1}\right) \ldots \tilde{\beta}_{d}\left(h_{1}, h_{2}, \ldots, h_{d-1} h_{d}, h_{d+1}\right) \tilde{\beta}_{d}\left(h_{1}, h_{2}, \ldots, h_{d}\right)}{\tilde{\beta}_{d}\left(h_{1} h_{2}, \ldots, h_{d+1}\right) \ldots \tilde{\beta}_{d}\left(h_{1}, h_{2}, \ldots, h_{d} h_{d+1}\right)} \\
& =\frac{(-1)^{g_{3} \cdots g_{d+1} k_{2}} \cdots(-1)^{g_{2} \cdots\left(g_{d-1}+g_{d}\right) g_{d+1} k_{1}}(-1)^{g_{2} \cdots g_{d} k_{1}}}{(-1)^{g_{3} \cdots g_{d+1}\left(k_{1}+k_{2}+g_{1} g_{2}\right)} \cdots(-1)^{g_{2} \cdots g_{d-1}\left(g_{d}+g_{d+1}\right) k_{1}}}=(-1)^{g_{1} g_{2} \ldots g_{d+1}},
\end{aligned}
$$

is true. Moreover, since $\mathcal{H}^{d}\left[Z_{n}^{T}, U(1)\right]=0$ for any odd dimension $d$, there is no further lower-dimensional topological phase of the $H=Z_{4}^{T}$-cocycle that we can append on the gapped boundary of an odd spacetime dimension $d$.
We find that the $d+1 \mathrm{D}$ bosonic SPTs with $Z_{2}^{T}$-symmetry (the bosonic topological superconductor of $G=Z_{2}^{T}$ ) have a $d \mathrm{D}$ symmetry-preserving surface deconfined $Z_{2}$ topologically ordered gauge theory, at least for $d \geq 3$. 
8. $3+1 / 2+1 \mathrm{D}$ bosonic topological superconductor $1 \rightarrow Z_{2} \rightarrow \operatorname{Pin}^{ \pm}(\infty) \rightarrow O(\infty) \rightarrow 1$ with $Z_{2}^{T}$ time-reversal symmetry: The $2+1 D Z_{2}^{K}$-gauge theory boundary of 3+1D bulk invariant $(-1)^{\int\left(w_{2}\right)^{2}}$ and $(-1)^{\int\left(w_{1}\right)^{4}+\left(w_{2}\right)^{2}}$

There is an additional $3+1 \mathrm{D}$ time-reversal symmetric bosonic topological superconductor (BTSC) beyond the previous $\mathcal{H}^{4}\left[Z_{2}^{T}, U_{T}(1)\right]=\mathbb{Z}_{2}$ class. It can be captured either within the group cohomology of $G \times S O_{\infty}$ [17] under $\mathcal{H}^{4}\left[Z_{2}^{T} \times S O(\infty), U_{T}(1)\right]=\left(\mathbb{Z}_{2}\right)^{2}$, [97] or the cobordism classification $\Omega_{O}^{4}[p t, U(1)]=\left(\mathbb{Z}_{2}\right)^{2}$ [19]. It gives rise to $3+1 \mathrm{D}$ bulk topological invariants $\mathrm{e}^{\mathrm{i} 2 \pi \int \frac{1}{2} w_{2}^{2}}=$ $(-1)^{\int\left(w_{2}\right)^{2}}$ or $(-1)^{\int\left(w_{1}\right)^{4}+\left(w_{2}\right)^{2}} \cdot w_{i}=w_{i}(T M)$ is the $i$ th Stiefel-Whitney class of a tangent bundle $T M$ over spacetime $M$. We would like to find out the surface $K$-gauge topological order through a short exact sequence.

First, notice that the spin group $\operatorname{Spin}(n)$ is the double cover of the special orthogonal group $S O(n)$. There exists a short exact sequence

$$
1 \rightarrow Z_{2} \rightarrow \operatorname{Spin}(n) \rightarrow S O(n) \rightarrow 1
$$

In our case, for the $3+1 \mathrm{D}$ bulk SPT invariant $(-1) \int\left(w_{2}\right)^{2}$ obtained through $G=Z_{2}^{T} \times S O(\infty)$ in $\mathcal{H}^{4}\left[Z_{2}^{T} \times S O(\infty)\right.$, $\left.U_{T}(1)\right]$, one may attempt to use the short exact sequence $1 \rightarrow Z_{2}^{K} \rightarrow Z_{2}^{T} \times \operatorname{Spin}(\infty) \rightarrow Z_{2}^{T} \times S O(\infty) \rightarrow 1$ to construct the surface $Z_{2}^{K}$-gauge theory. However, we suggest that the more proper way to consider a trivialization of the bulk BTSC is not based on $G=Z_{2}^{T} \times S O(\infty)$, but based on $G=O(\infty)$ via

$$
1 \rightarrow Z_{2}^{K} \rightarrow \operatorname{Pin}^{ \pm}(\infty) \rightarrow O(\infty) \rightarrow 1 .
$$

We can trivialize $(-1)^{\int\left(w_{2}\right)^{2}}$ on the $2+1 \mathrm{D}$ boundary by pulling $G=O(\infty)$ back to $H=\operatorname{Pin}^{+}(\infty)$. We can trivialize $(-1)^{\int\left(w_{1}\right)^{4}+\left(w_{2}\right)^{2}}$ on the $2+1 \mathrm{D}$ boundary by pulling $G=O(\infty)$ back to $H=\operatorname{Pin}^{-}(\infty)$. By picking a spin structure on the boundary, it means the boundary can have fermionic quasiparticles. The choice of spin structure can be viewed as a twisted version of $Z_{2}^{K}$ gauge theory.

We note that the $e^{f} m^{f}$ (and $e_{T}^{f} m_{T}^{f}$, as well) surface topological order first proposed in Ref. [39] on the surface of this $3+1 \mathrm{D} Z_{2}^{T}$-bosonic topological superconductor is also a $2+1 \mathrm{D}$ deconfined $Z_{2}$-gauge theory with quasiparticles of $Z_{2}$-gauge charge and $Z_{2}$-gauge flux, both with fermionic statistics.

\section{2+1/1+1D bosonic $0 \rightarrow Z_{2 N}^{K} \rightarrow Z_{4 N}^{H} \rightarrow Z_{2}^{G} \rightarrow 0$}

For $0 \rightarrow Z_{2 N}^{K} \stackrel{2}{\rightarrow} Z_{4 N}^{H} \stackrel{r}{\rightarrow} Z_{2}^{G} \rightarrow 0$, again we want to trivialize cocycle $\omega_{3}^{Z_{2}^{G}}\left(g_{a}, g_{b}, g_{c}\right)=(-1)^{g_{a} g_{b} g_{c}}$ to cochains. Generically, we can still reduce $(\bmod 4 N)$ to $(\bmod 4)$ in the exponent so that $\beta_{2}\left(h_{1}, h_{2}\right)=\exp \left[(2 \pi \mathrm{i} / 4)\left(\left[h_{1}\right]_{2}\right)\left(\left[h_{2}\right]_{4}\right)\right]$, or $\beta_{2}\left(h_{1}, h_{2}\right)=$ $\exp \left[(2 \pi \mathrm{i} / 4)\left(\left[h_{1}\right]_{4}\right)\left(\left[h_{2}\right]_{2}\right)\right]$ can be the successful split cochains.

\section{0. $2+1 / 1+1 \mathrm{D}$ bosonic $1 \rightarrow Z_{4}^{K} \rightarrow Q_{8}^{H} \rightarrow Z_{2}^{G} \rightarrow 1$}

Trivialize the three-cocycle in $\mathcal{H}^{3}\left[Z_{2}^{G}, U(1)\right]$. For the example that the $H=Q_{8}$ is a non-Abelian group, while $G=Z_{2}$, we write

$$
1 \rightarrow Z_{4}^{K} \rightarrow Q_{8}^{H} \stackrel{r}{\rightarrow} Z_{2}^{G} \rightarrow 1 .
$$

Again, $\omega_{3}^{Z_{2}^{G}}\left(g_{a}, g_{b}, g_{c}\right)=(-1)^{g_{a} g_{b} g_{c}}$.

Write the quaternion $Q_{8}=\langle x, y| x^{2}=y^{2}, x y x^{-1}=$ $\left.y^{-1}, x^{4}=y^{4}=1\right\rangle$ so that each element in the group we can write uniquely as $x^{g} y^{k}$, with $g \in\{0,1\}$ corresponding to $\{\{1, i,-1,-i\}, j\{1, i,-1,-i\}\}$ in $Z_{2}^{G}$ and $k \in\{0,1,2,3\}$ corresponding to $\{1, i,-1,-i\}$ in $Z_{4}^{K}$. Using $y x=x y^{-1}$ and $y^{-1} x=x y$, we can rewrite the group operation as

$x^{g_{1}} y^{k_{1}} x^{g_{2}} y^{k_{2}}=x^{g_{1}} x^{g_{2}} y^{(-1)^{g_{2}} k_{1}} y^{k_{2}}=x^{\left[g_{1}+g_{2}\right]_{2}} y^{\left[(-1)^{g_{2}} k_{1}+k_{2}+2 g_{1} g_{2}\right]_{4}}$.

We can write $h=(g, k)$ of $H$ as a doublet from $G$ and $K$, and then

$$
\begin{aligned}
h_{1} h_{2} & =\left(g_{1}, k_{1}\right) \cdot\left(g_{2}, k_{2}\right) \\
& =\left(g_{1}+g_{2},(-1)^{g_{2}} k_{1}+k_{2}+2 g_{1} g_{2}\right) \\
& \equiv\left[g_{1}+g_{2}, F\left(k_{1}, k_{2}, g_{1}, g_{2}\right)\right] .
\end{aligned}
$$

We find that the LHS technique in Appendix C works successfully. For the LHS technique of Appendix D 3, we look for

$$
\begin{aligned}
d_{2}: & \mathcal{H}^{1}\left(G, \mathcal{H}^{1}[K, U(1)]\right) \\
=\mathbb{Z}_{1} \rightarrow & \mathcal{H}^{3}\left(G, \mathcal{H}^{0}[K, U(1)]\right)=\mathcal{H}^{3}[G, U(1)]=\mathbb{Z}_{2} . \\
& \quad f: G \rightarrow \mathcal{H}^{1}[K, U(1)] \Rightarrow Z_{2}^{G} \rightarrow \mathbb{Z}_{4} .
\end{aligned}
$$

In this case, it is found that

$\beta_{2}\left(h_{1}, h_{2}\right)=\beta_{2}\left[\left(g_{1}, k_{1}\right),\left(g_{2}, k_{2}\right)\right]=f\left(g_{2}\right)^{k_{1}}=\mathrm{i}^{g_{2} k_{1}}$.

Here, $f\left(g_{2}^{-1}\right)$ corresponds to a $U(1)$ function labeled by $g_{2}$, and provides a $U(1)$ function via $f: G \rightarrow \mathcal{H}^{1}[K, U(1)]$. This $U(1)$ function depends on $k_{1} \in K$ for $\mathcal{H}^{1}[K, U(1)]$; thus, we have $\beta\left(h_{1}, h_{2}\right)=f\left(g_{2}^{-1}\right)\left(k_{1}\right)$. We look for the base of $\mathrm{i}$ because $\mathcal{H}^{1}[K, U(1)]=\mathbb{Z}_{4}$ is generated by $\mathrm{i}$ with $\mathrm{i}^{4}=1$.

We would like to find a two-cochain that satisfies the desired three-cocycle splitting property: 


$$
\begin{aligned}
\omega_{3}^{Q_{8}^{H}}\left(h_{a}, h_{b}, h_{c}\right) & =\omega_{3}^{Z_{2}^{G}}\left[r\left(h_{a}\right), r\left(h_{b}\right), r\left(h_{c}\right)\right] \\
& =(-1)^{r\left(h_{a}\right) r\left(h_{b}\right) r\left(h_{c}\right)}=(-1)^{g_{a} g_{b} g_{c}} \\
& =\left(\delta \beta_{2}\right)\left(h_{1}, h_{2}, h_{3}\right) .
\end{aligned}
$$

We write

$$
\begin{aligned}
\left(\delta \beta_{2}\right)\left(h_{1}, h_{2}, h_{3}\right) & =\frac{\beta_{2}\left(h_{2}, h_{3}\right) \beta_{2}\left(h_{1}, h_{2} h_{3}\right)}{\beta_{2}\left(h_{1} h_{2}, h_{3}\right) \beta_{2}\left(h_{1}, h_{2}\right)} \\
& =\frac{f\left(g_{3}\right)^{\left(k_{2}\right)} f\left(g_{2} g_{3}\right)^{\left(k_{1}\right)}}{f\left(g_{3}\right)^{\left[F\left(k_{1}, k_{2}, g_{1}, g_{2}\right)\right]} f\left(g_{2}\right)^{\left(k_{1}\right)}} .
\end{aligned}
$$

Recall that $f\left(g_{2} g_{3}\right)\left(k_{1}\right)$ is the cocycle of $\mathcal{H}^{1}[K, U(1)]$ with a power $k_{1}$. We should be able to rewrite $f\left(g_{2} g_{3}\right)$ based on the one-cocycle condition:

$$
\frac{f\left(g_{2}\right) f\left(g_{3}\right)}{f\left(g_{2} g_{3}\right)}=1 \Rightarrow f\left(g_{2} g_{3}\right)=f\left(g_{2}\right) f\left(g_{3}\right),
$$

so

$$
\begin{aligned}
\left(\delta \beta_{2}\right)\left(h_{1}, h_{2}, h_{3}\right) & =\frac{f\left(g_{3}\right)^{\left(k_{2}\right)} f\left(g_{2}\right)^{\left(k_{1}\right)} f\left(g_{3}\right)^{\left(k_{1}\right)}}{f\left(g_{3}\right)^{\left[F\left(k_{1}, k_{2}, g_{1}, g_{2}\right)\right]} f\left(g_{2}\right)^{\left(k_{1}\right)}} \\
& =\frac{f\left(g_{3}\right)^{\left(k_{2}\right)} f\left(g_{3}\right)^{\left(k_{1}\right)}}{f\left(g_{3}\right)^{\left[F\left(k_{1}, k_{2}, g_{1}, g_{2}\right)\right]}} \\
& =\frac{f\left(g_{3}\right)^{k_{2}} f\left(g_{3}\right)^{k_{1}}}{f\left(g_{3}\right)^{\left[(-1)^{g_{2}} k_{1}+k_{2}+2 g_{1} g_{2}\right]_{4}}} .
\end{aligned}
$$

Further computation shows, indeed,

$$
\begin{aligned}
& \left(\delta \beta_{2}\right)\left(h_{a}, h_{b}, h_{c}\right)=\frac{\beta_{2}\left(h_{b}, h_{c}\right) \beta_{2}\left(h_{a}, h_{b} h_{c}\right)}{\beta_{2}\left(h_{a} h_{b}, h_{c}\right) \beta_{2}\left(h_{a}, h_{b}\right)} \\
& =\frac{\mathbf{i}^{\left(k_{b} g_{c}\right)} \mathbf{i}^{k_{a}}\left[g_{b}+g_{c}\right]_{2}}{\mathbf{i}^{\left[k_{a}(-1)^{g_{b}}+k_{b}+2 g_{a} g_{b}\right]_{4} g_{c}} \mathbf{i}^{\left(k_{a} g_{b}\right)}}=(-1)^{g_{a} g_{b} g_{c}} .
\end{aligned}
$$

Because $\mathcal{H}^{2}\left[Q_{8}, U(1)\right]=0$, we do not have another lowerdimensional $1+1 \mathrm{D} Q_{8}$-topological state to stack on the boundary.

If we consider that the bulk is a fully gauged, topologically ordered state, this becomes a gapped boundary for a bulk $2+1 \mathrm{D}$ field theory of $\int(2 / 2 \pi) B d A+(1 / 2 \pi) A d A$.

\section{a. Degeneracy on a disk and an annulus: Partition functions $Z\left(D^{2} \times S^{\mathbf{1}}\right)$ and $Z\left(I^{\mathbf{1}} \times S^{\mathbf{1}} \times S^{\mathbf{1}}\right)$}

Following the setup in Appendix D 4 a, we put the $2+1 /$ $1+1 \mathrm{D} 1 \rightarrow Z_{4}^{K} \rightarrow Q_{8}^{H} \rightarrow Z_{2}^{G} \rightarrow 1$ construction of topological states on a spatial $D^{2}$ disk or an annulus $I^{1} \times S^{1}$ to count the degeneracy (GSD). Depending on gauging the global symmetry $K$ and $H$ or not, we have at least three types of theories. Since $K$ is a normal subgroup in $H$, we can label the $K$-holonomy in $H$. Thus, below, we write all holonomies $h$ in $H$. We consider the group homomorphisms:

$$
\begin{gathered}
Z_{4}^{K}=\left(\begin{array}{c}
1 \\
i \\
-1 \\
-i
\end{array}\right) \stackrel{1}{\rightarrow}\left(\begin{array}{c}
1 \\
i \\
-1 \\
-i
\end{array}\right) \subset Q_{8}^{H} \\
Q_{8}^{H}=\left(\begin{array}{c}
1, i,-1,-i \\
j, k,-j,-k
\end{array}\right) \rightarrow\left(\begin{array}{c}
1 \\
-1
\end{array}\right)=Z_{2}^{G} .
\end{gathered}
$$

We compute the partition function of Sec. IX A 5 on $Z\left(D^{2} \times S^{1}\right)$ to evaluate GSD on a spatial $D^{2}$ disk in Table IV.

The usual $1+1 \mathrm{D}$ topological gauge theory has its GSD on an $S^{1}$ ring and can be computed as $Z\left(S^{1} \times S^{1}\right)$ by

$$
\begin{aligned}
\mathrm{GSD} & =\left.\frac{1}{|H|} \sum_{h, t} 1\right|_{\text {if } h t=t h} \\
& =\frac{1}{|H|} \sum_{h}[\text { number of elements in the } \\
& \text { centralizer } \left.C_{H}(h) \text { of } h\right] \\
& =(\text { number of conjugacy classes of } H) \\
& =(\text { number of irrep of } H) \leq|H|,
\end{aligned}
$$

reduced to a smaller number than $|H|$. For $H=Q_{8}$, we have (number of conjugacy classes of $H$ ) $=$ (number of irrep of $H)=5<|H|=8$. The five conjugacy classes 1 ; $-1 ;\{i,-i\} ;\{j,-j\} ;$ and $\{k,-k\}$ yield five distinct holonomies for GSD $=5$ on $S^{1}$.

We find that the $h=1$ carries zero or an even $Z_{2}^{G}$ charge. The $h=i$ and $h=-i$ combined are also zero or an even $Z_{2}^{G}$ charge. Other sectors of $h$ carry an odd $Z_{2}^{G}$ charge. For theory (iii), when the $Z_{2}^{G}$ is gauged, the ground state for the whole system cannot carry an odd $Z_{2}^{G}$ charge; thus, $h=0$ or $h=i /-i \in H$ implies GSD $=2$ on a disk. An important remark is that we cannot regard the $1+1 \mathrm{D}$ anomalous

TABLE IV. For theory (ii), GSD $=4$ from the holonomy $h=1$, $i,-1,-i$ in $K$ and also in $H$. For the fully gauge theory (iii), $\mathrm{GSD}=2$ from the holonomy $h=1$ and $h=i /-i$. Here, $h=i$ and $h=-i$ each contributes $1 / 2$ state, and the i/ - i together act like a two-dimensional irreducible representation as a non-Abelian ground state. The setup and notations follow Appendix D 4 a.

Theory (i) Theory (ii) (third or Theory (iii) Disk $D^{2}$ (second boundary) fourth boundary) (fifth boundary)

\begin{tabular}{llll}
\hline GSD & 1 & 4 & 2 \\
\hline \hline
\end{tabular}


TABLE V. For theory (ii) without symmetry twist, GSD $=16$ from the holonomies of sectors $\left(h_{\text {in }}, h_{\text {out }}\right)$ with $h_{\text {in }}$, $h_{\text {out }} \in\{1, i,-1,-i\}$. For the theory (iii) fully gauge theory, $\mathrm{GSD}=8$ from the holonomies $\left(h_{\text {in }}, h_{\text {out }}\right)=(1,1) ;(-1,-1)$; $(1, i /-i) ;(-1, i /-i) ;(i /-i, 1) ;(i /-i,-1)$ and two more states from $(i /-i, i /-i)$. The setup and notations follow Appendix D 4 a.

Annulus Theory (i) Theory (ii) (third or Theory (iii) $S^{1} \times I^{1} \quad$ (second boundary) fourth boundary) (fifth boundary) GSD 1 16 8

$Q_{8}^{H}$ gauge theory as a usual $1+1 \mathrm{D}$ discrete gauge theorybecause the usual $1+1 \mathrm{D} Q_{8}$ gauge theory has GSD $=5$ on a $S^{1}$ ring. In our case, the $2+1 \mathrm{D}$ bulk plays an important role, which causes the GSD to decrease from five conjugacy classes to two conjugacy classes ( 1 and $\{i,-i\})$ of GSD $=2$ for theory (iii).

We compute the partition function of Sec. IX A 5 on $Z\left(I^{1} \times S^{1} \times S^{1}\right)$ to evaluate GSD on an annulus $I^{1} \times S^{1}$ in Table V.

Again, the $2+1 \mathrm{D}$ bulk plays an important role for the GSD reduction for theory (iii) from GSD $=\mid$ (number of ir rep of $H)\left.\right|^{2}=25$ to GSD $=8$ in Table V.

\section{1. $2+1 / 1+1 \mathrm{D}$ bosonic $1 \rightarrow Z_{2} \rightarrow D_{4} \rightarrow\left(Z_{2}\right)^{2} \rightarrow 1$}

We consider the construction $1 \rightarrow K=Z_{2} \rightarrow H=$ $D_{4} \rightarrow Q=\left(Z_{2}\right)^{2} \rightarrow 1$. The explicit group elements inside a quotient group can be written as

$$
\begin{aligned}
\frac{D_{4}}{Z_{2}}=\frac{D_{4}}{\left\{1, R^{2}\right\}} & =\left\{1\left\{1, R^{2}\right\}, \mathrm{X}\left\{1, R^{2}\right\}, R\left\{1, R^{2}\right\}, \mathrm{x} R\left\{1, R^{2}\right\}\right\} \\
& =\left(Z_{2}\right)^{2} .
\end{aligned}
$$

Here, we would like to trivialize the particular twisted three-cocycle of $G=\left(Z_{2}\right)^{2}$ :

$$
\begin{aligned}
\omega_{2}\left(g_{a}, g_{b}, g_{c}\right) & =\exp \left(\frac{\mathrm{i} 2 \pi}{2}\left[g_{a_{1}}\right]_{2}\left[g_{b_{2}}\right]_{2}\left[g_{c_{2}}\right]_{2}\right) \\
& =(-1)^{\left[g_{a_{1}}\right]_{2}\left[g_{b_{2}}\right]_{2}\left[g_{c_{2}}\right]_{2}},
\end{aligned}
$$

where $g_{a}=\left(g_{a_{1}}, g_{a_{2}}\right) \in G=\left(Z_{2}\right)^{2}$, and similarly for $g_{b}$, $g_{c}$. This cocycle is equivalent to $\mathrm{e}^{\mathrm{i} 2 \pi \int \frac{1}{2} a_{1} \cup a_{1} \cup a_{2}}$ with a cup product form of $a_{1} \cup a_{1} \cup a_{2}$, in $\mathcal{H}^{3}\left[\left(Z_{2}\right)^{2}, U(1)\right]$. The $a_{1}$ and $a_{2}$ here are $\mathbb{Z}_{2}$-valued one-cocycles in $\mathcal{H}^{1}\left(M^{3}, \mathbb{Z}_{2}\right)$ on the spacetime complex $M^{3}$. The boundary bosonic anomaly of $\left(Z_{2}\right)^{2}$-SPTs is explored in Ref. [96].

We can write $h=(g, k) \in H$, where $g \in G$ and $k \in K$. Let us write $h=\mathrm{x}^{a} R^{b} \in D_{4}$ in terms of a triplet, $h_{u}=\left(k_{u}, g_{u_{1}}, g_{u_{2}}\right) \in D_{4}$, such that

$$
\begin{aligned}
& \left(k_{u}, g_{u_{1}}, g_{u_{2}}\right) \cdot\left(k_{v}, g_{v_{1}}, g_{v_{2}}\right) \\
& \quad=\left(k_{u}+k_{v}+g_{u_{1}} g_{v_{2}}, g_{u_{1}}+g_{v_{1}}, g_{u_{2}}+g_{v_{2}}\right) .
\end{aligned}
$$

Note that the $R^{2}=(1,0,0) \in D_{4}$. The $D_{4} \rightarrow\left(Z_{2}\right)^{2}$ maps $h_{u}=\left(k_{u}, g_{u_{1}}, g_{u_{2}}\right) \in D_{4}$ to $\left(g_{u_{1}}, g_{u_{2}}\right) \in\left(Z_{2}\right)^{2}$. We can view the $k_{u}$ generates $R^{2}$ in $D_{4}$, while $g_{u_{1}}$ and $g_{u_{2}}$ generates $\mathrm{x}$ and $R$, respectively. We would like to split

$\begin{aligned} \omega_{3}^{H}\left(h_{u}, h_{v}, h_{w}\right) & =\omega_{3}^{G}\left[r\left(h_{u}\right), r\left(h_{v}\right), r\left(h_{w}\right)\right] \\ & =(-1)^{\left[g_{u_{1}}\right]_{2}\left[g_{v_{2}}\right]_{2}\left[g_{w_{2}}\right]_{2}}=\left(\delta \beta_{2}\right)\left(h_{u}, h_{v}, h_{w}\right),\end{aligned}$

into a two-cochain $\beta_{2}$. The LHS technique in Appendix D 3 suggests that we look for

$$
\begin{aligned}
d_{2}: \mathcal{H}^{1}\left(G, \mathcal{H}^{1}[K, U(1)]\right) & \rightarrow \mathcal{H}^{3}\left(G, \mathcal{H}^{0}[K, U(1)]\right) \\
\Rightarrow d_{2}: \mathcal{H}^{1}\left[\left(Z_{2}\right)^{2}, Z_{2}\right]=\left(\mathbb{Z}_{2}\right)^{2} & \rightarrow \mathcal{H}^{3}[G, U(1)]=\left(\mathbb{Z}_{2}\right)^{3},
\end{aligned}
$$

$f: G \rightarrow \mathcal{H}^{1}[K, U(1)] \Rightarrow\left(\mathbb{Z}_{2}\right)^{2} \rightarrow \mathcal{H}^{1}\left[Z_{2}^{K}, U(1)\right]=\mathbb{Z}_{2}$.

In this case, it is found that

$$
\begin{aligned}
\beta_{2}\left(h_{u}, h_{v}\right) & =\beta_{2}\left[\left(k_{u}, g_{u_{1}}, g_{u_{2}}\right),\left(k_{v}, g_{v_{1}}, g_{v_{2}}\right)\right] \\
& =f\left(g_{v}\right)^{k_{u}}=(-1)^{k_{u} g_{v_{2}}} .
\end{aligned}
$$

We can see that

$$
\begin{aligned}
\delta\left(\beta_{2}\right) & =\frac{\beta_{2}\left(h_{v}, h_{w}\right) \beta_{2}\left(h_{u}, h_{v} h_{w}\right)}{\beta_{2}\left(h_{u} h_{v}, h_{w}\right) \beta_{2}\left(h_{u}, h_{v}\right)} \\
& =\frac{(-1)^{k_{v} g_{w_{2}}(-1)^{k_{u}}\left(g_{v_{2}}+g_{w_{2}}\right)}}{(-1)^{\left(k_{u}+k_{v}+g_{u_{1}} g_{v_{2}}\right) g_{w_{2}}(-1)^{k_{u} g_{v_{2}}}}=(-1)^{g_{u_{1}} g_{v_{2}} g_{w_{2}}}} \\
& =\omega_{3}^{H}\left(h_{u}, h_{v}, h_{w}\right) .
\end{aligned}
$$

Similarly, it turns out that we can find another two-cochain $\beta_{2}\left(h_{u}, h_{v}\right)=(-1)^{k_{u} g_{v_{1}}}$ that splits a differ-

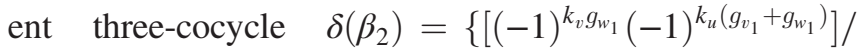

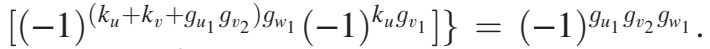

Since $\mathcal{H}^{2}\left[D_{4}, U(1)\right]=Z_{2}$, we can have two distinct classes of two-cochain differed by a two-cocycle $\omega_{2} \in$ $\mathcal{H}^{2}\left[D_{4}, U(1)\right]$, corresponding to a $1+1 \mathrm{D} D_{4}$-topological state on the boundary.

If we consider the bulk to be a fully gauged topologically ordered state, this becomes a gapped boundary for a bulk $2+1 \mathrm{D}$ field theory of $\int \sum_{I=1}^{2}(2 / 2 \pi) B_{I} d A_{I}+(1 / 2 \pi) A_{1} d A_{2}$.

\section{2. $1+1 / 0+1 \mathrm{D}$ bosonic $1 \rightarrow Z_{2} \rightarrow Q_{8} \rightarrow\left(Z_{2}\right)^{2} \rightarrow 1$}

Here, we would like to trivialize a particular twisted twococycle of $G=\left(Z_{2}\right)^{2}$ : 
$\omega_{2}\left(g_{a}, g_{b}\right)=\exp \left(\frac{\mathrm{i} 2 \pi}{2}\left[g_{a_{1}}\right]_{2}\left[g_{b_{2}}\right]_{2}\right)=(-1)^{\left[g_{a_{1}}\right]_{2}\left[g_{b_{2}}\right]_{2}}$,

where $g_{a}=\left(g_{a_{1}}, g_{a_{2}}\right) \in G=\left(Z_{2}\right)^{2}$, and similarly for $g_{b}$. This cocycle is equivalent to $\mathrm{e}^{\mathrm{i} 2 \pi \int \frac{1}{2} a_{1} \cup a_{2}}$ with a cup product form of $a_{1} \cup a_{2}$, in $\mathcal{H}^{2}\left[\left(Z_{2}\right)^{2}, U(1)\right]$. The $a_{1}$ and $a_{2}$ here are $\mathbb{Z}_{2}$-valued one-cocycles in $H^{1}\left(M^{2}, \mathbb{Z}_{2}\right)$ on the spacetime complex $M^{2}$.

We consider the construction $1 \rightarrow K=Z_{2} \rightarrow H=$ $Q_{8} \rightarrow G=\left(Z_{2}\right)^{2} \rightarrow 1$. The quotient group can be realized as $Q_{8} /\{1,-1\}=\left(Z_{2}\right)^{2}$. We write each element in the group $H=Q_{8}$ uniquely as $h=x^{h} y^{h^{\prime}}$ with $h \in\{0,1\}$ corresponding to $\{1\{1, i,-1,-i\}, j\{1, i,-1,-i\}\}$ and $h^{\prime} \in$ $\{0,1,2,3\}$ corresponding to $\{1, i,-1,-i\}$. By writing $h=x^{h} y^{h^{\prime}}$, the $h=1$ and $h^{\prime}=1$ correspond to two generators of the quotient group $G=\left(Z_{2}\right)^{2}$. Applying the relation $y x=x y^{-1}$ and $x^{2}=y^{2}$, we find $x^{h_{1}} y^{h_{1}{ }^{\prime}} x^{h_{2}} y^{h_{2}{ }^{\prime}}=$ $x^{\left[h_{1}+h_{2}\right]_{2}} y^{\left[h_{1}{ }^{\prime}(-1)^{h_{2}}+h_{2}{ }^{\prime}+2 h_{1} h_{2}\right]_{4}}$. We can rewrite

$$
\omega_{2}^{Q_{8}^{H}}\left(h_{a}, h_{b}\right)=\omega_{2}^{Z_{2}^{G}}\left[r\left(h_{a}\right), r\left(h_{b}\right)\right]=(-1)^{\left[h_{a}^{\prime}\right]_{2} h_{b}} .
$$

We claim that the above three-cocycle can be split by twocochains:

$$
\beta_{1}(h)=\beta_{1}\left(x^{h} y^{h^{\prime}}\right)=\mathrm{e}^{\mathrm{i} \frac{\pi}{2}\left(h+h^{\prime}\right)}=i^{\left(h+h^{\prime}\right)} .
$$

Indeed, we find it works:

$$
\begin{aligned}
& \left(\delta \beta_{1}\right)\left(h_{a}, h_{b}\right)=\frac{\beta_{1}\left(h_{a}\right) \beta_{1}\left(h_{b}\right)}{\beta_{1}\left(h_{a} h_{b}\right)}=\frac{i^{\left(h_{a}+h_{a}^{\prime}\right)} i^{\left(h_{b}+h_{b}^{\prime}\right)}}{i^{\left(\left[h_{a}+h_{b}\right]_{2}+\left[h_{a}^{\prime}(-1)^{h_{b}}+h_{b}^{\prime}+2 h_{a} h_{b}\right]_{4}\right)}} \\
& =\frac{i^{\left(\left[h_{a}\right]_{2}+\left[h_{a}^{\prime}\right]_{4}\right)_{i}} \mathbf{i}\left(\left[h_{b}\right]_{2}+\left[h_{b}^{\prime}\right]_{4}\right)}{i^{\left(\left[h_{a}+h_{b}\right]_{2}+\left[h_{a}^{\prime}(-1)^{h_{b}}+h_{b}^{\prime}+2 h_{a} h_{b}\right]_{4}\right)}} \\
& =\frac{i^{\left(\left[h_{a}^{\prime}\right]_{4}\right)} \mathbf{i}^{\left(\left[h_{b}^{\prime}\right]_{4}\right)}}{i^{\left(\left[h_{a}^{\prime}(-1)^{h_{b}}+h_{b}^{\prime}\right]_{4}\right)}}=i^{h_{a}^{\prime}\left[1-(-1)^{\left.h_{b}\right]}\right.}=(-1)^{h_{a}^{\prime} h_{b}} \\
& =(-1)^{\left[h_{a}^{\prime}\right]_{2} h_{b}}=\omega_{2}^{Q_{8}^{H}}\left(h_{a}, h_{b}\right) .
\end{aligned}
$$

There are various legal one-cochains that trivialize the $G$ two-cocycle as a two-coboundary in $H$, such as $\beta_{1}(h)=\beta_{1}\left(x^{h} y^{h^{\prime}}\right)=i^{\left(h+h^{\prime}\right)}, i^{\left(h-h^{\prime}\right)}, i^{\left(-h+h^{\prime}\right)}, i^{\left(-h-h^{\prime}\right)}$. These one-cochains can be differed by a one-cocycle $\omega_{1}^{H}$ in $H=Q_{8}$, such that $\omega_{1}^{H}(h) \in \mathcal{H}^{1}\left[Q_{8}, U(1)\right]=\left(\mathbb{Z}_{2}\right)^{2}$; thus, they differ by a $0+1 \mathrm{D}$ topological state on the boundary. Indeed, the one-cocycle $\omega_{1}^{H}$ can be

$$
\omega_{1}\left(x^{h} y^{h^{\prime}}\right)=(-1)^{h},(-1)^{h^{\prime}},(-1)^{h+h^{\prime}} .
$$

One can check if the following is true:

$$
\left(\delta \omega_{1}\right)\left(h_{a}, h_{b}\right)=\frac{\omega_{1}\left(h_{a}\right) \omega_{1}\left(h_{b}\right)}{\omega_{1}\left(h_{a} h_{b}\right)}=1 .
$$

All these one-cochains $\beta_{1}\left(x^{h} y^{h^{\prime}}\right)=i^{\left(h+h^{\prime}\right)}, i^{\left(h-h^{\prime}\right)}, i^{\left(-h+h^{\prime}\right)}$, $i^{\left(-h-h^{\prime}\right)}$ are differed by each other via stacking $0+1 \mathrm{D}$ topological states labeled by one-cocycle $\omega_{1}=$ $(-1)^{h},(-1)^{h^{\prime}},(-1)^{h+h^{\prime}} \in \mathcal{H}^{1}\left[Q_{8}, U(1)\right]=Z_{2} \times Z_{2}$.

The LHS technique in Appendix D 3 suggests that we look for

$$
\begin{aligned}
d_{2}: \mathcal{H}^{0}\left(G, \mathcal{H}^{1}[K, U(1)]\right) & \rightarrow \mathcal{H}^{2}\left(G, \mathcal{H}^{0}[K, U(1)]\right) \\
\Rightarrow d_{2}: \mathcal{H}^{0}\left[\left(Z_{2}\right)^{2}, Z_{2}\right]=\mathbb{Z}_{2} & \rightarrow \mathcal{H}^{2}\left[\left(\mathbb{Z}_{2}\right)^{2}, U(1)\right]=\mathbb{Z}_{2} .
\end{aligned}
$$

$f: G \rightarrow \mathcal{H}^{1}[K, U(1)] \Rightarrow\left(\mathbb{Z}_{2}\right)^{2} \rightarrow \mathcal{H}^{1}\left[Z_{2}^{K}, U(1)\right]=\mathbb{Z}_{2}$.

In this case, it suggested that $\beta_{1}(h)=\beta_{1}[(g, k)]$ can be written as a base of $(-1)$, but we found the solution for a base of $i$ instead. So, the LHS technique is not helpful here.

If we consider the bulk as a fully gauged topologically ordered state, this becomes a gapped boundary for a bulk $1+1 \mathrm{D}$ field theory of $\int \sum_{I=1}^{2}(2 / 2 \pi) B_{I} d A_{I}+\frac{1}{\pi} A_{1} A_{2}$.

\section{3. $1+1 / 0+1 D$ bosonic $1 \rightarrow Z_{2} \rightarrow D_{4} \rightarrow\left(Z_{2}\right)^{2} \rightarrow 1$}

Here, we would like to trivialize a particular twisted twococycle of $G=\left(Z_{2}\right)^{2}$ based on $1 \rightarrow Z_{2}^{K} \rightarrow D_{4} \stackrel{r}{\rightarrow}\left(Z_{2}\right)^{2} \rightarrow 1$,

$\omega_{2}\left(g_{a}, g_{b}\right)=\exp \left(\frac{i 2 \pi}{2}\left[g_{a_{1}}\right]_{2}\left[g_{b_{2}}\right]_{2}\right)=(-1)^{\left[g_{a_{1}}\right]_{2}\left[g_{b_{2}}\right]_{2}}$,

where $g_{a}=\left(g_{a_{1}}, g_{a_{2}}\right) \in G=\left(Z_{2}\right)^{2}$, and similarly for $g_{b}$. This cocycle is equivalent to $\mathrm{e}^{\mathrm{i} 2 \pi \int \frac{1}{2} a_{1} \cup a_{2}}$ with a cup product form of $a_{1} \cup a_{2}$, in $\mathcal{H}^{2}\left[\left(Z_{2}\right)^{2}, U(1)\right]$. The $a_{1}$ and $a_{2}$ here are $\mathbb{Z}_{2}$-valued one-cochains in $H^{1}\left(M^{2}, \mathbb{Z}_{2}\right)$ on the spacetime complex $M^{2}$.

Here, $D_{4}$ is a dihedral group of order 8 , namely, $\left|D_{4}\right|=8$. Write the dihedral group $D_{4}=\langle x, R| x^{2}=R^{4}=1, x R x=$ $\left.R^{-1}\right\rangle$ so that each element in the group we can write uniquely as $x^{a} R^{b}$ with $a \in\{0,1\}$ and $b \in\{0,1,2,3\}$. The quotient group is

$$
\begin{aligned}
\frac{D_{4}}{Z_{2}} & =\frac{D_{4}}{\left\{1, R^{2}\right\}}=\left\{1\left\{1, R^{2}\right\}, x\left\{1, R^{2}\right\}, R\left\{1, R^{2}\right\}, x R\left\{1, R^{2}\right\}\right\} \\
& =\left(Z_{2}\right)^{2}
\end{aligned}
$$

We find the split one-cochain as $\beta_{1}(h)=(-1)^{\mathrm{f}(h)}$. This onecochain satisfies the desired two-cocycle splitting property. Here, we can define the function $f$ :

$$
\begin{aligned}
f(1) & =f(x)=f(R)=f(x R)=0 \in Z_{2}^{K}, \\
f\left(R^{2}\right) & =f\left(x \cdot R^{2}\right)=f\left(R \cdot R^{2}\right)=f\left(x R \cdot R^{2}\right)=1 \in Z_{2}^{K} .
\end{aligned}
$$


Let us write $h=x^{a} R^{b} \in D_{4}$ in terms of a doublet $h=(k, g)$, or a more precise triplet, $h_{u}=\left(k_{u}, g_{u_{1}}, g_{u_{2}}\right) \in D_{4}$, such that $\left(k_{u}, g_{u_{1}}, g_{u_{2}}\right) \cdot\left(k_{v}, g_{v_{1}}, g_{v_{2}}\right)=\left(k_{u}+k_{v}+g_{u_{1}} g_{v_{2}}, g_{u_{1}}+g_{v_{1}}, g_{u_{2}}+g_{v_{2}}\right)$. Note that the $R^{2}=(1,0,0) \in D_{4}$. The $D_{4} \rightarrow\left(Z_{2}\right)^{2}$ maps $h_{u}=\left(k_{u}, g_{u_{1}}, g_{u_{2}}\right) \in D_{4}$ to $\left(g_{u_{1}}, g_{u_{2}}\right) \in\left(Z_{2}\right)^{2}$, so that

$$
\begin{gathered}
f(h)=f\left(x^{a} R^{b}\right)=\frac{b-[b]_{2}}{2}=k_{u}= \begin{cases}1, & \text { if } b=2,3 . \\
0, & \text { if } b=0,1 .\end{cases} \\
\beta_{1}\left(h_{u}\right)=(-1)^{f\left(h_{u}\right)}=(-1)^{k_{u}} .
\end{gathered}
$$

We can see that, indeed,

$$
\begin{aligned}
\delta\left(\beta_{1}\right) & =\frac{\beta_{1}\left(h_{u}\right) \beta_{1}\left(h_{v}\right)}{\beta_{1}\left(h_{u} h_{v}\right)}=\frac{(-1)^{k_{u}}(-1)^{k_{v}}}{(-1)^{k_{u}+k_{v}+g_{u_{1}} g_{v_{2}}}} \\
& =(-1)^{g_{u_{1}} g_{v_{2}}}=\omega_{2}^{G}\left[r\left(h_{u}\right), r\left(h_{v}\right)\right]=\omega_{2}^{H}\left(h_{u}, h_{v}\right) .
\end{aligned}
$$

The LHS technique in Appendix D 3 suggests that we look for

$$
\begin{gathered}
d_{2}: \mathcal{H}^{0}\left(G, \mathcal{H}^{1}[K, U(1)]\right) \rightarrow \mathcal{H}^{2}\left(G, \mathcal{H}^{0}[K, U(1)]\right) \\
\Rightarrow d_{2}: \mathcal{H}^{0}\left[\left(Z_{2}\right)^{2}, Z_{2}\right]=\mathbb{Z}_{2} \rightarrow \mathcal{H}^{2}[G, U(1)]=\mathbb{Z}_{2} . \\
f: G \rightarrow \mathcal{H}^{1}[K, U(1)] \Rightarrow\left(\mathbb{Z}_{2}\right)^{2} \rightarrow \mathcal{H}^{1}\left[Z_{2}^{K}, U(1)\right]=\mathbb{Z}_{2},
\end{gathered}
$$

with a base of $(-1)$. In this case, it is true that $\beta_{1}\left(h_{u}\right)=$ $\beta_{1}\left(\left(k_{u}, g_{u_{1}}, g_{u_{2}}\right)\right)=(-1)^{k_{u}}$.

If we consider the bulk to be a fully gauged topologically ordered state, this becomes a gapped boundary for a bulk $1+1 \mathrm{D}$ field theory of $\int \sum_{I=1}^{2}(2 / 2 \pi) B_{I} d A_{I}+(1 / \pi) A_{1} A_{2}$.

\section{4. $2+1 / 1+1 D$ bosonic $1 \rightarrow Z_{2} \rightarrow D_{4} \times Z_{2} \rightarrow\left(Z_{2}\right)^{3} \rightarrow 1$}

Here, we would like to trivialize the three-cocycle of a cup product form $\mathrm{e}^{\mathrm{i} 2 \pi \int \frac{1}{2} a_{1} \cup a_{2} \cup a_{3}}$ in $\mathcal{H}^{3}\left[\left(Z_{2}\right)^{3}, U(1)\right]$ with $a_{i} \in$ $H^{1}\left(M^{3}, Z_{2}\right)$ of an $M^{3}$-spacetime complex, via $1 \rightarrow Z_{2}^{K} \rightarrow$ $D_{4} \times Z_{2} \stackrel{r}{\rightarrow}\left(Z_{2}\right)^{3} \rightarrow 1$. The particular twisted three-cocycle of $G=\left(Z_{2}\right)^{3}$ that we would like to focus on is

$$
\omega_{3}\left(g_{a}, g_{b}, g_{c}\right)=(-1)^{\left[g_{a_{1}}\right]_{2}\left[g_{b_{2}}\right]_{2}\left[g_{c_{3}}\right]_{2}}
$$

where $g_{a}=\left(g_{a_{1}}, g_{a_{2}}, g_{a_{3}}\right) \in G=\left(Z_{2}\right)^{3}$, and similarly for $g_{b}$ and $g_{c}$. The boundary bosonic anomaly of $\left(Z_{2}\right)^{3}$-SPTs is explored in Ref. [96].

Here, $D_{4}$ is a dihedral group of order 8, namely, $\left|D_{4}\right|=8$. We write the dihedral group $D_{4}=\langle\mathrm{x}, R| \mathrm{x}^{2}=$ $\left.R^{4}=1, \mathrm{x} R \mathrm{x}=R^{-1}\right\rangle$ so that we can write each element in the group uniquely as $\mathrm{x}^{a} R^{b}$ with $a \in\{0,1\}$ and $b \in\{0,1,2,3\}$. Indeed, the group homomorphism $D_{4} \times$ $Z_{2} \rightarrow\left(Z_{2}\right)^{3}$ can be understood from a reduced map: $D_{4} \rightarrow$ $\left(Z_{2}\right)^{2}$. We only need to understand the short exact sequence
$1 \rightarrow Z_{2}^{K} \rightarrow D_{4} \stackrel{r}{\rightarrow}\left(Z_{2}\right)^{2} \rightarrow 1$ in Appendix D 13. Namely, we can take the $Z_{2}$ in $D_{4} \times Z_{2}$ mapping directly to the third $Z_{2}$ component in $\left(Z_{2}\right)^{3}$, while we only have to specify $D_{4} \stackrel{r}{\rightarrow}\left(Z_{2}\right)^{2} \quad$ such that $\left\{1\left\{1, R^{2}\right\}, x\left\{1, R^{2}\right\}, R\left\{1, R^{2}\right\}\right.$, $\left.x R\left\{1, R^{2}\right\}\right\} \stackrel{r}{\rightarrow}\left(Z_{2}\right)^{2}$. Meanwhile, the normal subgroup $Z_{2}^{K}$ can be viewed as $\left\{1, R^{2}\right\}$ in $D_{4}$.

We denote the group elements of $h_{u} \in D_{4} \times Z_{2}$ as $\left(k_{u}, g_{u_{1}}, g_{u_{2}}, g_{u_{3}}\right)$, where $\left(k_{u}, g_{u_{1}}, g_{u_{2}}\right) \in D_{4}$, and $g_{u_{3}} \in Z_{2}$, such that $\left(k_{u}, g_{u_{1}}, g_{u_{2}}\right) \cdot\left(k_{v}, g_{v_{1}}, g_{v_{2}}\right)=\left(k_{u}+k_{v}+g_{u_{1}} g_{v_{2}}, g_{u_{1}}+\right.$ $\left.g_{v_{1}}, g_{u_{2}}+g_{v_{2}}\right)$. Following the construction in the previous Appendix D 13, we note that the $R^{2}=(1,0,0) \in D_{4}$. The $D_{4} \times Z_{2} \rightarrow\left(Z_{2}\right)^{3}$ maps $h_{u}=\left(k_{u}, g_{u_{1}}, g_{u_{2}}, g_{u_{3}}\right) \in D_{4} \times Z_{2}$ to $\left(g_{u_{1}}, g_{u_{2}}, g_{u_{3}}\right) \in\left(Z_{2}\right)^{3}$. We propose that this two-cochain satisfies the desired three-cocycle splitting property:

$$
\beta_{2}\left(h_{u}, h_{v}\right)=(-1)^{\mathrm{f}\left(h_{u}\right) g_{v_{3}}}=(-1)^{k_{u} g_{v_{3}}} .
$$

We can, indeed, show

$$
\begin{aligned}
& \left(\delta \beta_{2}\right)\left(h_{u}, h_{v}, h_{w}\right)=\frac{\beta_{2}\left(h_{v}, h_{w}\right) \beta_{2}\left(h_{u}, h_{v} h_{w}\right)}{\beta_{2}\left(h_{u} h_{v}, h_{w}\right) \beta_{2}\left(h_{u}, h_{v}\right)} \\
& =\frac{(-1)^{k_{v} g_{w_{3}}}(-1)^{k_{u}\left(g_{v_{3}}+g_{w_{3}}\right)}}{(-1)^{\left(k_{u}+k_{v}+g_{u_{1}} g_{v_{2}}\right) g_{w_{3}}}(-1)^{k_{u} g_{v_{3}}}} \\
& =(-1)^{g_{u_{1}} g_{v_{2}} g_{w_{3}}} \\
& =\omega_{3}^{G}\left[r\left(h_{u}\right), r\left(h_{v}\right), r\left(h_{w}\right)\right] \\
& =\omega_{3}^{H}\left(h_{u}, h_{v}, h_{w}\right) \text {. }
\end{aligned}
$$

The LHS technique in Appendix D 3 also gives the correct hint.

If we consider the bulk to be a fully gauged topologically ordered state, this becomes a gapped boundary for a bulk $2+1 \mathrm{D}$ field theory of $\int \sum_{I=1}^{3}(2 / 2 \pi) B_{I} d A_{I}+$ $\left(1 / \pi^{2}\right) A_{1} A_{2} A_{3}$.

\section{5. $3+1 / 2+1 \mathrm{D}$ bosonic $1 \rightarrow Z_{2} \rightarrow D_{4} \times\left(Z_{2}\right)^{2} \rightarrow$ $\left(Z_{2}\right)^{4} \rightarrow 1$ and $d+1 / d \mathrm{D}$ bosonic $1 \rightarrow Z_{2} \rightarrow$ $D_{4} \times\left(Z_{2}\right)^{d-1} \rightarrow\left(Z_{2}\right)^{d+1} \rightarrow 1$}

We can easily generalize from Appendixes D 13 and D 14 to any dimension. For example, based on a $3+1 / 2+1 \mathrm{D}$ bosonic $1 \rightarrow Z_{2} \rightarrow D_{4} \times\left(Z_{2}\right)^{2} \rightarrow\left(Z_{2}\right)^{4} \rightarrow 1$ construction, we can trivialize the four-cocycle of a cup product form $\mathrm{e}^{\mathrm{i} 2 \pi \int \frac{1}{2} a_{1} \cup a_{2} \cup a_{3} \cup a_{4}}$ in $\mathcal{H}^{4}\left[\left(Z_{2}\right)^{4}, U(1)\right]$, here, $a_{i} \in H^{1}\left(M^{4}, Z_{2}\right)$ of an $M^{4}$-spacetime complex. We denote the group elements of $h_{u} \in D_{4} \times\left(Z_{2}\right)^{2}$ as $\left(k_{u}, g_{u_{1}}, g_{u_{2}}, g_{u_{3}}, g_{u_{4}}\right)$, where $\left(k_{u}, g_{u_{1}}, g_{u_{2}}\right) \in D_{4}$, and $\left(g_{u_{3}}, g_{u_{4}}\right) \in\left(Z_{2}\right)^{2}$. We can define a three-cochain in $H$,

$$
\beta_{3}\left(h_{u}, h_{v}, h_{w}\right)=(-1)^{\mathrm{f}\left(h_{u}\right) g_{v_{3}} g_{w_{4}}}=(-1)^{k_{u} g_{v_{3}} g_{w_{4}},}
$$

that indeed splits a nontrivial four-cocycle 


$$
\begin{aligned}
& \left(\delta \beta_{3}\right)\left(h_{u}, h_{v}, h_{w}, h_{z}\right) \\
& =\frac{\beta_{3}\left(h_{v}, h_{w}, h_{z}\right) \beta_{3}\left(h_{u}, h_{v} h_{w}, h_{z}\right) \beta_{3}\left(h_{u}, h_{v}, h_{w}\right)}{\beta_{3}\left(h_{u} h_{v}, h_{w}, h_{z}\right) \beta_{3}\left(h_{u}, h_{v}, h_{w} h_{z}\right)}
\end{aligned}
$$

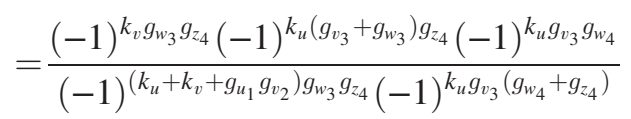

$$
\begin{aligned}
& =(-1)^{g_{u_{1}} g_{v_{2}} g_{w_{3}} g_{z_{4}}} \\
& =\omega_{4}^{G}\left[r\left(h_{u}\right), r\left(h_{v}\right), r\left(h_{w}\right), r\left(h_{z}\right)\right]=\omega_{4}^{H}\left(h_{u}, h_{v}, h_{w}, h_{z}\right) .
\end{aligned}
$$

In general, based on a $d+1 / d \mathrm{D}$ bosonic construction via $1 \rightarrow Z_{2} \rightarrow D_{4} \times\left(Z_{2}\right)^{d-1} \rightarrow\left(Z_{2}\right)^{d+1} \rightarrow 1$, we can trivialize the $d+1$-cocycle of a cup product form $\mathrm{e}^{\mathrm{i} 2 \pi \int \frac{1}{2} a_{1} \cup a_{2} \cup \ldots \cup a_{d+1}}$ in $\mathcal{H}^{d+1}\left[\left(Z_{2}\right)^{d+1}, U(1)\right]$. We denote the group elements of $h_{u} \in D_{4} \times\left(Z_{2}\right)^{d-1}$ as $\left(k_{u}, g_{u_{1}}, g_{u_{2}}, g_{u_{3}}, \ldots, g_{u_{d+1}}\right)$, where $\left(k_{u}, g_{u_{1}}, g_{u_{2}}\right) \in D_{4}$, and $\left(g_{u_{3}}, g_{u_{4}}, \ldots, g_{u_{d+1}}\right) \in\left(Z_{2}\right)^{d-1}$. We can write down the $d$-cochain

$$
\begin{aligned}
\beta_{d}\left(h_{u}, h_{v}, h_{w}, h_{z}, \ldots\right) & =(-1)^{\mathrm{f}\left(h_{u}\right) g_{v_{3}} g_{w_{4}} g_{z_{5}} \ldots g_{\cdot d+1}} \\
& =(-1)^{k_{u} g_{v_{3}} g_{w_{4}} g_{z_{5}} \ldots g \cdot d+1}
\end{aligned}
$$

that splits a nontrivial $d+1$-cocycle in $\mathcal{H}^{d+1}\left[\left(Z_{2}\right)^{d+1}\right.$, $U(1)]$ :

$$
\begin{aligned}
& \omega_{d+1}^{G}\left[r\left(h_{u}\right), r\left(h_{v}\right), r\left(h_{w}\right), r\left(h_{z}\right), \ldots\right] \\
& \quad=\omega_{d+1}^{H}\left(h_{u}, h_{v}, h_{w}, h_{z}, \ldots\right)=(-1)^{g_{u_{1}} g_{v_{2}} g_{w_{3}} g_{z_{4}} \ldots g_{\cdot d+1}} .
\end{aligned}
$$

Again the LHS technique in Appendix D 3 also gives the correct hint.

If we consider the bulk to be a fully gauged topologically ordered state, this becomes a gapped boundary for a bulk $d+1 \mathrm{D}$ field theory of $\int \sum_{I=1}^{d+1}(2 / 2 \pi) B_{I} d A_{I}+$ $\left(1 /(\pi)^{d}\right) A_{1} A_{2} \ldots A_{d+1}$.

$$
\begin{gathered}
\text { 16. } 2+1 / 1+1 \mathrm{D} \text { bosonic } \\
1 \rightarrow\left(Z_{2}\right)^{2} \rightarrow D_{4} \times Z_{2} \rightarrow\left(Z_{2}\right)^{2} \rightarrow 1
\end{gathered}
$$

Here, we would like to trivialize a particular twisted twococycle of $G=\left(Z_{2}\right)^{2}$ in $\mathcal{H}^{3}\left[\left(Z_{2}\right)^{2}, U(1)\right]$,

$$
\begin{aligned}
\omega_{3}\left(g_{a}, g_{b}, g_{c}\right) & =\exp \left(\frac{\mathrm{i} 2 \pi}{2}\left[g_{a_{1}}\right]_{2}\left[g_{b_{2}}\right]_{2}\left[g_{c_{2}}\right]_{2}\right) \\
& =(-1)^{\left[g_{a_{1}}\right]_{2}\left[g_{b_{2}}\right]_{2}\left[g_{c_{2}}\right]_{2}},
\end{aligned}
$$

where $g_{a}=\left(g_{a_{1}}, g_{a_{2}}\right) \in G=\left(Z_{2}\right)^{2}$, and similarly for $g_{b}$ and $g_{c}$. The boundary bosonic anomaly of $\left(Z_{2}\right)^{2}$-SPTs is explored in Ref. [96].

The idea is extending the $1+1 \mathrm{D}$ example of Appendix D 13 via $1 \rightarrow Z_{2}^{K} \rightarrow D_{4} \stackrel{r}{\rightarrow}\left(Z_{2}\right)^{2} \rightarrow 1$ in the normal subgroup side by $Z_{2}$, and we seek, for a realization in $2+1 \mathrm{D}$,

$$
1 \rightarrow\left(Z_{2}\right)^{2} \rightarrow D_{4} \times Z_{2} \stackrel{r}{\rightarrow}\left(Z_{2}\right)^{2} \rightarrow 1 .
$$

Since we have discussed that, in Appendix D 11, the $2+1 \mathrm{D}$ example of

$$
1 \rightarrow Z_{2}^{K} \rightarrow D_{4} \stackrel{r}{\rightarrow}\left(Z_{2}\right)^{2} \rightarrow 1
$$

already trivializes the three-cocycle of a cup product form $\mathrm{e}^{\mathrm{i} 2 \pi \int \frac{1}{2} a_{1} \cup a_{2} \cup a_{2}}$ in $\mathcal{H}^{3}\left[\left(Z_{2}\right)^{2}, U(1)\right]$, then we can simply take $D_{4} \times Z_{2} \stackrel{r}{\rightarrow}\left(Z_{2}\right)^{2}$ as the combination of $D_{4} \stackrel{r}{\rightarrow}\left(Z_{2}\right)^{2}$ and $Z_{2} \stackrel{r}{\rightarrow} 1$. We denote the group elements of $h_{u} \in$ $D_{4} \times Z_{2}$ as $\left(k_{u}, g_{u_{1}}, g_{u_{2}}, g_{u_{3}}\right)$, where $\left(k_{u}, g_{u_{1}}, g_{u_{2}}\right) \in D_{4}$, and $g_{u_{3}} \in Z_{2}$, such that $\left(k_{u}, g_{u_{1}}, g_{u_{2}}\right) \cdot\left(k_{v}, g_{v_{1}}, g_{v_{2}}\right)=$ $\left(k_{u}+k_{v}+g_{u_{1}} g_{v_{2}}, g_{u_{1}}+g_{v_{1}}, g_{u_{2}}+g_{v_{2}}\right)$. We propose the split two-cochain

$$
\beta_{2}\left(h_{u}, h_{v}\right)=(-1)^{k_{u} g_{v_{2}}} .
$$

We can see that

$$
\begin{aligned}
& \left(\delta \beta_{2}\right)=\frac{\beta_{2}\left(h_{v}, h_{w}\right) \beta_{2}\left(h_{u}, h_{v} h_{w}\right)}{\beta_{2}\left(h_{u} h_{v}, h_{w}\right) \beta_{2}\left(h_{u}, h_{v}\right)} \\
& =\frac{(-1)^{k_{v} g_{w_{2}}}(-1)^{k_{u}\left(g_{v_{2}}+g_{w_{2}}\right)}}{(-1)^{\left(k_{u}+k_{v}+g_{u_{1}} g_{v_{2}}\right) g_{w_{2}}(-1)^{k_{u} g_{v_{2}}}}}=(-1)^{g_{u_{1}} g_{v_{2}} g_{w_{2}}} \\
& =\omega_{3}^{G}\left[r\left(h_{u}\right), r\left(h_{v}\right), r\left(h_{w}\right)\right]=\omega_{3}^{H}\left(h_{u}, h_{v}, h_{w}\right) .
\end{aligned}
$$

The LHS technique in Appendix D 3 gives the correct hint. Basically, this shows the same result as in Appendix D 11.

\section{3+1/2+1D bosonic $1 \rightarrow\left(Z_{2}\right) \rightarrow D_{4} \rightarrow\left(Z_{2}\right)^{2} \rightarrow 1$}

Here, we would like to trivialize a particular twisted fourcocycle of $G=\left(Z_{2}\right)^{2}$ in $\mathcal{H}^{4}\left[\left(Z_{2}\right)^{2}, U(1)\right]$,

$$
\begin{aligned}
\omega_{4}\left(g_{a}, g_{b}, g_{c},, g_{d}\right) & =\exp \left(\frac{\mathrm{i} 2 \pi}{2}\left[g_{a_{1}}\right]_{2}\left[g_{b_{2}}\right]_{2}\left[g_{c_{2}}\right]_{2}\left[g_{d_{2}}\right]_{2}\right) \\
& =(-1)^{\left[g_{a_{1}}\right]_{2}\left[g_{b_{2}}\right]_{2}\left[g_{c_{2}}\right]_{2}\left[g_{d_{2}}\right]_{2}} .
\end{aligned}
$$

We consider the construction via $1 \rightarrow Z_{2} \rightarrow D_{4} \rightarrow$ $\left(Z_{2}\right)^{2} \rightarrow 1$. Following the earlier definition of $D_{4}$ group elements, we propose the split three-cochain

$$
\beta_{3}\left(h_{u}, h_{v}, h_{w}\right)=(-1)^{\mathrm{f}\left(h_{u}\right) g_{v_{2}} g_{w_{2}}}=(-1)^{k_{u} g_{v_{2}} g_{w_{2}}} .
$$

We can check explicitly that the three-cochain splits the four-cocycle in $H$ : 


$$
\begin{aligned}
& \left(\delta \beta_{3}\right)\left(h_{u}, h_{v}, h_{w}, h_{z}\right) \\
& =\frac{\beta_{3}\left(h_{v}, h_{w}, h_{z}\right) \beta_{3}\left(h_{u}, h_{v} h_{w}, h_{z}\right) \beta_{3}\left(h_{u}, h_{v}, h_{w}\right)}{\beta_{3}\left(h_{u} h_{v}, h_{w}, h_{z}\right) \beta_{3}\left(h_{u}, h_{v}, h_{w} h_{z}\right)}
\end{aligned}
$$

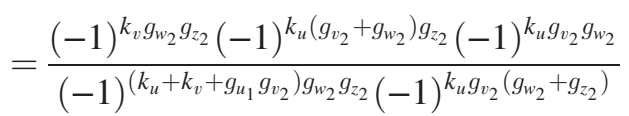

$$
\begin{aligned}
& =(-1)^{g_{u_{1}} g_{v_{2}} g_{w_{2}} g_{z_{2}}} \\
& =\omega_{4, \mathrm{II}}^{G}=\omega_{4}^{G}\left[r\left(h_{u}\right), r\left(h_{v}\right), r\left(h_{w}\right), r\left(h_{z}\right)\right] \\
& =\omega_{4}^{H}\left(h_{u}, h_{v}, h_{w}, h_{z}\right) \text {. }
\end{aligned}
$$

If we consider the bulk to be a fully gauged topologically ordered state, this becomes a gapped boundary for a field theory of $\int \sum_{I=1}^{2}(2 / 2 \pi) B_{I} d A_{I}+\left[1 / 2(\pi)^{2}\right] A_{1} A_{2} d A_{2}$.

\section{3+1/2 + 1D bosonic $1 \rightarrow Z_{2} \rightarrow D_{4} \times Z_{2} \rightarrow\left(Z_{2}\right)^{3} \rightarrow 1$}

Here, we would like to trivialize the four-cocycle of a particular twisted four-cocycle of $G=\left(Z_{2}\right)^{3}$ in $\mathcal{H}^{4}\left[\left(Z_{2}\right)^{3}\right.$, $U(1)]$

$$
\begin{aligned}
\omega_{4}\left(g_{a}, g_{b}, g_{c}, g_{d}\right) & =\exp \left(\frac{\mathrm{i} 2 \pi}{2}\left[g_{a_{1}}\right]_{2}\left[g_{b_{2}}\right]_{2}\left[g_{c_{3}}\right]_{2}\left[g_{d_{3}}\right]_{2}\right) \\
& =(-1)^{\left[g_{a_{1}}\right]_{2}\left[g_{b_{2}}\right]_{2}\left[g_{c_{3}}\right]_{2}\left[g_{d_{3}}\right]_{2}} .
\end{aligned}
$$

We consider the construction via $1 \rightarrow Z_{2} \rightarrow D_{4} \times Z_{2} \rightarrow$ $\left(Z_{2}\right)^{3} \rightarrow 1$. Following the earlier definition of $D_{4}$ group elements, we propose the split three-cochain

$$
\beta_{3}\left(h_{u}, h_{v}, h_{w}\right)=(-1)^{\mathrm{f}\left(h_{u}\right) g_{v_{3}} g_{w_{3}}}=(-1)^{k_{u} g_{v_{3}} g_{w_{3}}} .
$$

We can check explicitly that the three-cochain splits the four-cocycle in $H$ :

$$
\begin{aligned}
& \left(\delta \beta_{3}\right)\left(h_{u}, h_{v}, h_{w}, h_{z}\right) \\
& =\frac{\beta_{3}\left(h_{v}, h_{w}, h_{z}\right) \beta_{3}\left(h_{u}, h_{v} h_{w}, h_{z}\right) \beta_{3}\left(h_{u}, h_{v}, h_{w}\right)}{\beta_{3}\left(h_{u} h_{v}, h_{w}, h_{z}\right) \beta_{3}\left(h_{u}, h_{v}, h_{w} h_{z}\right)}
\end{aligned}
$$

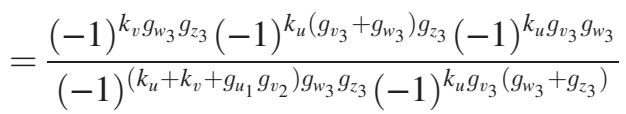

$$
\begin{aligned}
& =(-1)^{g_{u_{1}} g_{v_{2}} g_{w_{3}} g_{z_{3}}} \\
& =\omega_{4, \mathrm{III}}^{G}=\omega_{4}^{G}\left[r\left(h_{u}\right), r\left(h_{v}\right), r\left(h_{w}\right), r\left(h_{z}\right)\right] \\
& =\omega_{4}^{H}\left(h_{u}, h_{v}, h_{w}, h_{z}\right) \text {. }
\end{aligned}
$$

If we consider the bulk to be a fully gauged topologically ordered state, this becomes a gapped boundary for a field theory of $\int \sum_{I=1}^{3}(2 / 2 \pi) B_{I} d A_{I}+\left(1 / 2(\pi)^{2}\right) A_{1} A_{2} d A_{3}$.

\section{9. $2+1 / 1+1 \mathrm{D}$ to $d+1 / d \mathrm{D}$ bosonic $1 \rightarrow Z_{N} \rightarrow U(1) \rightarrow U(1) \rightarrow 1:$ Symmetry-enforced gapless boundaries protected by perturbative anomalies}

It is tempting to ask for the construction of a $2+1 / 1+1 \mathrm{D}$ topological state via

$$
1 \rightarrow Z_{N} \rightarrow U(1) \rightarrow U(1) \rightarrow 1
$$

where the bulk has $2+1 \mathrm{D} U(1)$ SPTs obtained from $\mathcal{H}^{3}[U(1), U(1)]=\mathbb{Z}$, while the boundary has $1+1 \mathrm{D}$ SETs with a $U(1)$ global symmetry and an emergent exact $Z_{N}$ gauge symmetry.

Of course, this kind of group extension along the boundary is possible, in general. But then the boundary theory is a $1+1 \mathrm{D}$ theory with a $U(1)$ global symmetry that has a perturbative 't Hooft anomaly [29]. As in 't Hooft's original work on such matters, this obstructs the possibility of symmetrically gapping the boundary theory. Similar remarks apply for any even $d$-dimensional spacetime of the boundary theory.

\section{0. $6+1 / 5+1 \mathrm{D}$ bosonic$$
1 \rightarrow Z_{2} \rightarrow U(1) \times S O(\infty) \rightarrow U(1) \times S O(\infty) \rightarrow 1:
$$$$
\text { Surface topological order and global mixed }
$$ gauge-gravitational anomaly}

The previous Appendix D19 discusses the $U(1)$ anomaly on the boundary of SPTs obtained from the group cohomology $\mathcal{H}^{d+1}[U(1), U(1)]=\mathbb{Z}$ of symmetry group $G=U(1)$ for the even $d$. However, there are $U(1)$ anomalies beyond the $\mathcal{H}^{d+1}[G, U(1)]$ but within $\mathcal{H}^{d+1}[G \times S O(\infty), U(1)]$ [17]. One example is the $3+$ 1D perturbative mixed gauge-gravity anomaly $[17,28]$ on the surface of $4+1 \mathrm{D} U(1)$-SPTs, characterized by

$$
\exp \left(\mathrm{i} 2 \pi \int \frac{1}{3} \frac{A}{2 \pi} p_{1}\right)
$$

where $A$ is a $U(1)$ one-form gauge field and $p_{1}$ is the first Pontryagin class of the tangent bundle of a spacetime manifold. In this example, the boundary has a perturbative 't Hooft anomaly [29] and, therefore, again cannot be symmetrically gapped.

Another SPT theory with a $6+1 \mathrm{D}$ bulk $/ 5+1 \mathrm{D}$ boundary dimension can have a $\mathbb{Z}_{2}$ anomaly [within $\left.\mathcal{H}^{7}[U(1) \times S O(\infty), U(1)]=(\mathbb{Z})^{2} \times \mathbb{Z}_{2}\right]$, labeled by the bulk topological invariant [17] on a seven-manifold $M^{7}$ :

$\exp \left(i 2 \pi \int_{M^{7}} \frac{1}{2} w_{2} w_{3} \frac{d A}{2 \pi}\right)=\exp \left(i 2 \pi \int_{M^{7}} \frac{1}{2} w_{2} w_{3} c_{1}\right)$,

where $w_{i}$ is the $i$ th Stieffel-Whitney class. Here, $w_{i}$ is a cohomology class with mod 2 coefficients. We can write 
$w_{i}=w_{i}\left(T M^{7}\right)$ of the spacetime tangent bundle $T M^{7}$. This $\mathbb{Z}_{2}$ class indicates a nonperturbative global mixed gaugegravitational anomaly from a continuous group $U(1)$. We suggest that the $5+1 \mathrm{D} Z_{2}$ gauge theory can be a boundary topological order, via the construction $1 \rightarrow Z_{2} \rightarrow U(1) \times$ $S O(\infty) \rightarrow U(1) \times S O(\infty) \rightarrow 1$, as a symmetry-preserving gapped boundary. The $U(1)$ in the total group $H$ is the double cover of that $U(1)$ in the quotient group $G$. The boundary field theory could be

$$
\sum_{\frac{b \in C^{4}\left((\partial M)^{6}, \mathbb{Z}_{2}\right)}{a \in C^{1}\left((\partial M)^{6}, \mathbb{Z}_{2}\right)}} \exp \left[i 2 \pi \int_{(\partial M)^{6}} \frac{1}{2}\left((b \delta a)+w_{2} w_{3} a+b c_{1}\right)\right] .
$$

The $C^{d}\left(\mathcal{M}, \mathbb{Z}_{n}\right)$ contains all $d$-cochains of $\mathbb{Z}_{n}$ values assigned to a $d$-simplex on a triangulated manifold $\mathcal{M}$. Here, $a$ is a one-cochain and $b$ is a four-cochain; both are integers with $\mathbb{Z}_{2}$ values. It is basically a $5+1 \mathrm{D} Z_{2}$ gauge theory. The "gauge transformations" are

$$
\begin{aligned}
w_{2} & \rightarrow w_{2}+\delta \alpha, \quad w_{3} \rightarrow w_{3}+\delta \beta, \quad \lambda \equiv \alpha \delta \beta+w_{2} \beta+\alpha w_{3}, \\
b & \rightarrow b+\lambda, \quad c_{1} \rightarrow c_{1}+\delta \gamma, \quad a \rightarrow a-\gamma .
\end{aligned}
$$

Here $\lambda, \alpha, \beta$, and $\gamma$ are four-cochain, one-cochain, twocochain, and one-cochain, respectively, all in $\mathbb{Z}_{2}$ values. We have the gauge transformation $w_{2} w_{3} \rightarrow w_{2} w_{3}+\delta \lambda=$ $w_{2} w_{3}+w_{2} \delta \beta+\delta \alpha w_{3}+\delta \alpha \delta \beta$, because the SW classes satisfy $\delta w_{2}=\delta w_{3}=0$. The whole partition function with bulk and boundary theories together is gauge invariant. Since both $a$ and $b$ are $\mathbb{Z}_{2}$-valued cochains, coupled to $w_{2}$, $w_{3}$, and $c_{1}$ of the background $U(1)$ probed field, we can regard the $5+1 \mathrm{D}$ surface theory as a $Z_{2}$ gauge theory.

\section{1. $2+1 \mathrm{D} / 1+1 \mathrm{D}$ bosonic topological insulator $1 \rightarrow$ $Z_{2}^{K} \rightarrow U(1) \rtimes Z_{2}^{T} \rightarrow U(1) \rtimes Z_{2}^{T} \rightarrow 1$ and $2+1 \mathrm{D} / 1+1 \mathrm{D}$ bosonic topological superconductor of $Z_{2}^{K} \rtimes Z_{2}^{T}$ : Spontaneous $G$-symmetry breaking of boundary deconfined $K$-gauge theory}

The bosonic SPT with symmetry group $G=U(1) \rtimes Z_{2}^{T}$ is called a bosonic topological insulator (BTI). In $2+1 \mathrm{D}$, we can obtain these SPTs from the group cohomology $\mathcal{H}^{3}\left[U(1) \rtimes Z_{2}^{T}, U(1)\right]=\mathbb{Z}_{2}$. Let us focus on the nontrivial $\mathbb{Z}_{2}$ class; the bulk field theory on a three-manifold $M^{3}$ is described by $[17,20]$

$$
\exp \left(i 2 \pi \int_{M^{3}} \frac{1}{2} w_{1} \frac{d A}{2 \pi}\right)=\exp \left(i 2 \pi \int_{M^{3}} \frac{1}{2} w_{1} c_{1}\right)
$$

The boundary field theory can be described by

$$
\sum_{\frac{\phi \in C^{0}\left((\partial M)^{2}, \mathbb{Z}_{2}\right)}{a \in C^{1}\left((\partial M)^{2}, \mathbb{Z}_{2}\right)}} \exp \left[i 2 \pi \int_{(\partial M)^{2}} \frac{1}{2}\left(\phi \delta a+w_{1} a+\phi c_{1}\right)\right],
$$

where $\phi$ is a zero-cochain and $a$ is a one-cochain, both in $\mathbb{Z}_{2}$ values. The "gauge transformations" are

$$
\begin{aligned}
w_{1} & \rightarrow w_{1}+\delta \alpha, \quad \phi \rightarrow \phi+\alpha, \\
c_{1} & \rightarrow c_{1}+\delta \gamma, \quad a \rightarrow a-\gamma .
\end{aligned}
$$

Here, $\alpha$ and $\gamma$ are a zero-cochain and one-cochain in $\mathbb{Z}_{2}$ values. The $c_{1}$ is an integral two-cochain defined the same as in the previous Appendix D 20. The boundary theory shows a $K=Z_{2}$ gauge theory in $1+1 \mathrm{D}$ coupled to $w_{1}$ and $c_{1}$. In terms of the $U(1)$-field $A$, we have the gauge transformation $A \rightarrow A+2 \pi \gamma$. This establishes our construction:

$$
1 \rightarrow Z_{2}^{K} \rightarrow U(1) \rtimes Z_{2}^{T} \rightarrow U(1) \rtimes Z_{2}^{T} \rightarrow 1 .
$$

For this $Z_{2}^{K}$ gauge theory, there are a few topologically distinct sectors and gauge-invariant operators, as shown in Table VI: (1) The trivial sector is 1, with trivial quantum number $U(1)$ charge 0 and $T=+1$. (2) The $Z_{2}^{K}$ gauge charge as an $e$-sector corresponds to the line operator $e^{i \pi \int[a+(A / 2 \pi)]}$. Each of two ends of such an open line $e^{i \pi \int_{x_{1}}^{x_{2}}[a+(A / 2 \pi)]}$ has an $e$-particle $\left(Z_{2}^{K}\right.$ gauge charge $\left.e\right)$. Each of the two ends must attach with a $1 / 2 U(1)$ charge, due to its attachment to the $U(1)$-field $A$. Thus, the $e$-particle has quantum number $U(1)$ charge $1 / 2$ and $T=+1$. (3) The $Z_{2}^{K}$ gauge flux as an $m$-sector corresponds to the line operator $e^{i \pi\left[\phi\left(x_{1}\right)-\phi\left(x_{2}\right)+\int_{x_{1}}^{x_{2}} w_{1}\right]}$, where the vortex $e^{i \pi \phi}$ is an $m$-instanton insertion operator. Similarly, each of the two ends of the open line must attach with an $m$ instanton with an eigenvalue of $T=-1$, due to $w_{1}$. The $m$ instanton has a trivial eigenvalue of $U(1)$, namely 0 .

If we put either $2+1 \mathrm{D}$ SPT on a spatial disk with a circular boundary, and if the boundary $Z_{2}$ gauge theory is deconfined, there are twofold degenerate ground states, labeled by a trivial (no) holonomy and a nontrivial

TABLE VI. The quantum numbers $[U(1)$ charge and $T]$ of the $U(1)$ symmetry and $Z_{2}^{T}$ time-reversal symmetry here are meant to be associated to $e$-particle local excitations and $m$-instantons (the second column), not to the entire line operators (the first column).

\begin{tabular}{lccc}
\hline \hline Operators & $\begin{array}{c}\text { Sectors (fractional } \\
\text { objects) }\end{array}$ & $\begin{array}{c}U(1) \\
\text { charge }\end{array}$ & $\begin{array}{c}T \\
\text { eigenvalue }\end{array}$ \\
\hline 1 & Trivial (none) & 0 & 1 \\
$e^{i \pi \int[a+(A / 2 \pi)]}$ & $\begin{array}{c}Z_{2} \text { gauge charge } \\
(e \text {-particle) }\end{array}$ & $1 / 2$ & 1 \\
$e^{i \pi\left[\phi\left(x_{1}\right)-\phi\left(x_{2}\right)+\int_{x_{1}}^{x_{2}} w_{1}\right]}$ & $\begin{array}{c}Z_{2} \text { gauge flux } \\
(m \text {-instanton) }\end{array}$ & 0 & -1 \\
\hline \hline
\end{tabular}


holonomy of a $Z_{2}$ gauge charge (e-particle) winding an odd number of times, along the circular boundary.

Note that the SPTs with a smaller symmetry group $Z_{2} \rtimes$ $Z_{2}^{T}$ also render the same class, due to $\mathcal{H}^{3}\left[Z_{2} \rtimes Z_{2}^{T}, U(1)\right]=$ $\left(\mathbb{Z}_{2}\right)^{2}$; one of the $\mathbb{Z}_{2}$ class coincides with $\mathcal{H}^{3}[U(1) \rtimes$ $\left.Z_{2}^{T}, U(1)\right]=\mathbb{Z}_{2}$. The SPT invariant for that $\mathbb{Z}_{2}$ class in $\mathcal{H}^{3}\left[Z_{2} \rtimes Z_{2}^{T}, U(1)\right]=\left(\mathbb{Z}_{2}\right)^{2}$ is

$$
\exp \left[\mathrm{i} 2 \pi \int_{M^{3}} \frac{1}{2} w_{1}\left(a_{1}\right)^{2}\right]
$$

with a $\mathbb{Z}_{2}$-valued one-cochain $a_{1}$. This implies that the boundary physics of $2+1 \mathrm{D} U(1) \rtimes Z_{2}^{T}$ SPTs can be understood in terms of that of $2+1 \mathrm{D} Z_{2} \rtimes Z_{2}^{T}$ SPTs. Even if the Coleman-Mermin-Wager theorem protects the continuous $U(1)$-symmetry against spontaneous symmetry breaking, we may break $U(1)$ explicitly down to $Z_{2}$. The same physics is valid for both $U(1) \rtimes Z_{2}^{T}$ BTI and $Z_{2} \rtimes Z_{2}^{T}$ SPTs.

For the $K=Z_{2}$ deconfined gauge theory on the $1+1 \mathrm{D}$ boundary of the above $U(1) \rtimes Z_{2}^{T}$ and $Z_{2} \rtimes Z_{2}^{T}$ SPTs, we should have no spontaneous symmetry breaking, neither on the $U(1)$ (supposing that Coleman-Mermin-Wager theorem still holds) nor on the $Z_{2}$ [because $U(1) \rtimes Z_{2}^{T}$ SPTs and $Z_{2} \rtimes Z_{2}^{T}$ SPTs have the same physics]. It is likely that the boundary has spontaneous symmetry breaking on the timereversal symmetry $Z_{2}^{T}$. Below, we provide arguments to support that the time-reversal symmetry $Z_{2}^{T}$ is spontaneously broken at the boundary.

\section{Spontaneous global symmetry breaking of} boundary $K$-gauge theory: $Z_{2}^{G}$-symmetry breaking on $2+1 \mathrm{D} Z_{2}$-SPT's boundary vs $Z_{2}^{T}$-symmetry breaking on

\section{$2+1 \mathrm{D} U(1) \rtimes Z_{2}^{T}$-SPT's and $Z_{2} \rtimes Z_{2}^{T}$-SPT's boundaries for $K=Z_{2}^{K}$}

Here, we would like to show that $1+1 \mathrm{D}$ deconfined $K$-gauge theories with symmetry $G$ on the boundary of $2+1 \mathrm{D}$ bulk $G$-SPTs can actually be spontaneous global $G$-symmetry-breaking states. Some examples are in order.

(1) Our first example is already mentioned in the main text, in Sec. III C, as well as Appendixes A $2 \mathrm{~d}$ and D 4. Consider the $1+1 \mathrm{D}$ boundary of $2+1 \mathrm{D} Z_{2^{-}}$ SPTs under the construction $0 \rightarrow Z_{2}^{K} \rightarrow Z_{4}^{H} \rightarrow Z_{2}^{G} \rightarrow 0$. This $\mathbb{Z}_{2}$-valued three-cocycle of bulk SPTs is equivalent to $\mathrm{e}^{\mathrm{i} 2 \pi \int \frac{1}{2} a_{1} \cup a_{1} \cup a_{1}}=(-1) \int a_{1} \cup a_{1} \cup a_{1}$ with a cup product form of $a_{1} \cup a_{1} \cup a_{1}$, in $\mathcal{H}^{3}\left[Z_{2}, U(1)\right]$. The $a_{1}$ is a $\mathbb{Z}_{2}$-valued one-cochain. Through a field theory analysis, we can find a gauge-invariant partition function for the bulk on $M^{3}$ and boundary on $(\partial M)^{2}$. The boundary $Z_{2}^{K}$ gauge theory has a minimal coupling to the bulk fields, and its partition function is

$$
\sum_{\frac{\left.\phi \in C^{0}(\partial M)^{2}, \mathbb{Z}_{2}\right) .}{a \in C^{1}\left((\partial M)^{2}, \mathbb{Z}_{2}\right)}} \exp \left[i 2 \pi \int_{(\partial M)^{2}} \frac{1}{2}\left(\phi \delta a+\phi\left(a_{1}\right)^{2}+a a_{1}\right)\right] .
$$

Here, $\phi$ and $a$ are $\mathbb{Z}_{2}$-valued zero-cochain and onecochain fields, respectively. The boundary has a spin1 electric gauge charge excitation associated to the $a$, and a spin-0 magnetic instanton associated to the $\phi$. The gauge-invariant vortex operator has a nonzero vacuum expectation value with respect to ground states:

$$
\begin{aligned}
& \left\langle e^{i \pi\left[\phi\left(x_{1}\right)-\phi\left(x_{2}\right)+\int_{x_{1}}^{x_{2}} a_{1}\right]}\right\rangle \\
& =\left\langle\Psi_{\mathrm{gs}}\left|e^{i \pi\left[\phi\left(x_{1}\right)-\phi\left(x_{2}\right)+\int_{x_{1}}^{x_{2}} a_{1}\right]}\right| \Psi_{\mathrm{gs}}\right\rangle=\mathrm{const}
\end{aligned}
$$

The const. stands some constant value. This statement shows the same physics as Eq. (3.21)'s $\left\langle\Psi_{\mathrm{gs}}( \pm)\left|X_{i+1 / 2} X_{j+1 / 2}\right| \Psi_{\mathrm{gs}}( \pm)\right\rangle=1$. The spin-0 vortex operator that is odd under $Z_{2}^{G}$-symmetry has a real expectation value, and its two-point function develops a long-range order. This implies that $Z_{2}^{G}$-symmetry is violated. Thus, the ground states of $Z_{2}^{K}$-gauge theory have spontaneous $Z_{2}^{G}$-symmetry breaking.

(2) The second example is the main example of Appendix D 21, the $1+1 \mathrm{D}$ boundary of 2+1D $U(1) \rtimes Z_{2}^{T}$ SPTs under the construction $1 \rightarrow Z_{2}^{K} \rightarrow U(1) \rtimes Z_{2}^{T} \rightarrow$ $U(1) \rtimes Z_{2}^{T} \rightarrow 1$. Again, the gauge-invariant vortex operator (see Table VI) has a nonzero vacuum expectation value with respect to ground states:

$$
\begin{aligned}
& \left\langle e^{i \pi\left[\phi\left(x_{1}\right)-\phi\left(x_{2}\right)+\int_{x_{1}}^{x_{2}} w_{1}\right]}\right\rangle \\
& =\left\langle\Psi_{\mathrm{gs}}\left|e^{i \pi\left[\phi\left(x_{1}\right)-\phi\left(x_{2}\right)+\int_{x_{1}}^{x_{2}} w_{1}\right]}\right| \Psi_{\mathrm{gs}}\right\rangle=\mathrm{const}
\end{aligned}
$$

The vortex operator that is odd under $Z_{2}^{T}$-symmetry has a real expectation value, and its two-point function develops a long-range order. This implies that $Z_{2}^{T}$-symmetry is violated. Thus, the ground states have spontaneous $Z_{2}^{T}$-symmetry breaking. For the third example, we can also show that the $1+1 \mathrm{D}$ boundary of $2+1 \mathrm{D} Z_{2} \rtimes Z_{2}^{T}$-SPTs under the construction $1 \rightarrow Z_{2}^{K} \rightarrow Z_{2} \rtimes Z_{2}^{T} \rightarrow Z_{2} \rtimes Z_{2}^{T} \rightarrow 1$ has the same two-point function as Eq. (D93) and develops a long-range order for $Z_{2}^{T}$-symmetry-odd vortex operators. Thus, the ground states of $Z_{2}^{K}$-gauge theory have spontaneous $Z_{2}^{T}$-symmetry breaking.

To summarize, the above field theory analysis suggests that the ground states of $1+1 \mathrm{D}$ deconfined $K$-gauge theory of $2+1 \mathrm{D} G$-SPTs have spontaneous $G$-symmetry breaking. We expect that both its deconfined gauge theory and confined gauge theory have spontaneous $G$-symmetry 
breaking, with crossover to each other without phase transitions, similar to the physics in Appendix A 2 d.

\section{3. $1+1 / 0+1 \mathrm{D}$ bosonic $1 \rightarrow Z_{2} \rightarrow S U(2) \rightarrow S O(3) \rightarrow 1$}

In $1+1 \mathrm{D}$, we have a nontrivial bosonic SPT state predicted by $\mathcal{H}^{2}[S O(3), U(1)]=\mathbb{Z}_{2}$. This nontrivial class is exactly a $1+1 \mathrm{D}$ Haldane spin chain protected by the global symmetry $S O(3)$. For example, it is well known that the $1+1 \mathrm{D}$ Haldane SPT state is the ground state of the AKLT spin chain Hamiltonian:

$H=\sum_{j}\left[\frac{1}{2}\left(\vec{S}_{j} \cdot \vec{S}_{j+1}+\frac{1}{3}\left(\vec{S}_{j} \cdot \vec{S}_{j+1}\right)^{2}\right)+1 / 3\right]$.

Each site $j$ has a Hilbert space of a spin-1 degree of freedom, and the spin- 1 operator $\vec{S}_{j}$ acts on each site $j$. The particular choice of Hamiltonian prefers the lowest-energy ground state such that the spin-1 on each site splits to two spin- $1 / 2$ qubits, and the neighbor spin- $1 / 2$ spins between two sites have a total spin-0 singlet pairing. In a closed chain, we have a gapped state with a unique ground state. In an infinite-size open chain, we have a gapped state with two dangling spin- $1 / 2$ qubits at the two ends, where the two dangling spin- $1 / 2$ of a spin- 0 singlet and three spin- 1 triplet states become fourfold degenerate.

However, we can lift the fourfold degeneracy of a $1+$ 1D open chain by adding two spin- $1 / 2$ qubits at the two ends. Formally, this is achieved by trivializing the twococycle of $\mathcal{H}^{2}[S O(3), U(1)]$ by lifting $S O(3)$ to $S U(2)$ via

$$
1 \rightarrow Z_{2} \rightarrow S U(2) \rightarrow S O(3) \rightarrow 1 .
$$

The bulk topological term $(-1)^{\int w_{2}[S O(3)]}$ of the second SW class of principle $G=S O(3)$-bundle becomes trivial when we lift $S O(3)$ to the $S U(2)$-bundle. The unique gapped ground state is achieved when we introduce the edge Hamiltonian term pairing each of the old dangling spin$1 / 2$ qubits to the two newly added spin- $1 / 2$ qubits, such that the low-energy ground state favors the singlet spin- 0 pairing sectors at the two ends [98].

The LHS technique in Appendix D 3 suggests that we look for

$$
\begin{aligned}
& d_{2}: \mathcal{H}^{0}\left(G, \mathcal{H}^{1}[K, U(1)]\right) \rightarrow \mathcal{H}^{2}\left(G, \mathcal{H}^{0}[K, U(1)]\right) \\
& \Rightarrow d_{2}: \mathcal{H}^{0}\left[S O(3), Z_{2}\right]=\mathbb{Z}_{2} \rightarrow \mathcal{H}^{2}[S O(3), U(1)]=\mathbb{Z}_{2},
\end{aligned}
$$

$$
f: G \rightarrow \mathcal{H}^{1}[K, U(1)] \Rightarrow S O(3) \rightarrow \mathcal{H}^{1}\left[Z_{2}^{K}, U(1)\right]=\mathbb{Z}_{2},
$$

with a one-cochain of a suggested base of $(-1)$.

\section{APPENDIX E: SYMMETRY-BREAKING GAPPED BOUNDARIES OR INTERFACES: COMMENTS AND CRITERIA}

The main focus of this article is a new approach to define a gapped interface via "symmetry extension": lifting $G$ to a larger group $H$, as described in Sec. VIII and Appendix D, that trivializes the $G$-cocycle to define a lower-dimensional gapped boundary prescribed by the split $H$-cochain. On the other hand, there is another, more familiar approach for a gapped interface, known in the literature as "symmetry breaking." Namely, the global or gauge symmetries are spontaneously or explicitly broken, described in Sec. VIII A. For a finite group $G$, when the symmetry breaking does not produce gapless Goldstone bosons, the boundary can be gapped. Phenomenologically, one can achieve symmetry breaking through the Higgs effect or through interactions such as sine-Gordon cosine potentials.

The global symmetry-breaking mechanism is well known in the fields of topological insulators and SPTs. For example, we can add a ferromagnet on the boundary of topological insulators to break time-reversal global symmetry to obtain a gapped anomalous surface quantum Hall state. The gauge-symmetry-breaking mechanism is also known in the literature. The gapped boundary or interface criteria studied by Haldane [99], Kapustin-Saulina [78], Kitaev-Kong [79], Lan-Wang-Wen [80,84], and many others can be viewed as gauge-symmetry breaking [80-82, 84] or the Anderson-Higgs effect.

In particular, let us look at the symmetry-breaking mechanism in $2+1 \mathrm{D}$ Abelian bulk topological phases for simplicity. The bulk phase can be described by an Abelian Chern-Simons theory with an action $S_{\text {bulk }}=$ $\left(K_{I J} / 4 \pi\right) \int a_{I} \wedge d a_{J}$ under a symmetric integral bilinear matrix $K$ and, locally, some one-form gauge fields $a$. The usual gapless boundary action is a $K$-matrix Luttinger liquid or a doubled-version chiral boson theory $S_{\partial}=$ $(1 / 4 \pi) \int d t d x\left(K_{I J} \partial_{t} \Phi_{I} \partial_{x} \Phi_{J}-V_{I J} \partial_{x} \Phi_{I} \partial_{x} \Phi_{J}\right)$ with a nonuniversal velocity matrix $V_{I J}$ and some scalar modes $\Phi$. The gapped boundary conditions can be achieved through a set of sine-Gordon cosine terms $\int d t d x \sum_{a} g_{a} \cos \left(\ell_{a, I} \cdot \Phi_{I}\right)$ as a strong coupling $g_{a} \gg 1$ limit. Notice that the gapping cosine term indeed breaks the symmetry of $\Phi_{I} \rightarrow \Phi_{I}+\eta$ for some constant $\eta$. Here, the broken symmetry can be global symmetry [100] or gauge symmetry [78-82], depending on the context.

The simplest example is that $G^{\prime}=1$ is a trivial group containing only the identity element. $G^{\prime} \rightarrow G$ is a map that the identity in $G^{\prime}$ maps to the identity in $G$. This can be regarded as breaking $G$ to nothing in $G^{\prime}$. There are $G$-cocycles assigned in the bulk, but the boundary becomes a trivial cocycle or cochain 1 in $G^{\prime}$. In terms of the inhomogeneous cochain, $\beta_{d-1}^{G^{\prime}}=1$. The $G$-cocycle $\omega_{d}^{G}\left(g_{01}, \ldots, g_{d-1 d}\right)$ that touches any boundary link, say, $g_{01}^{\prime}$, must have $\omega_{d}^{G}\left[\imath\left(g_{01}^{\prime}\right)=1, \ldots, g_{d-1 d}\right]=1$. This type of boundary 
condition works for any bulk defined by any discrete group $G$ with any cocycle. The usual way that one would describe it is that the $G$ is spontaneously broken to nothing along the boundary.

More generally, the symmetry-breaking mechanism involves breaking $G$-topological phases of group $G$ down to a subgroup $G^{\prime}$ :

$$
G^{\prime} \stackrel{\imath}{\rightarrow} G
$$

viewed through the injective map $t$. If $G^{\prime}$ is a subgroup of $G$, then we can define the symmetry-breaking gapped boundary of $G$-topological phases, if the $G^{\prime}$-cocycle becomes a $G^{\prime}$-coboundary [with a similar expression as in Eq. (D4)]

$$
\begin{aligned}
\omega_{d}^{G}\left[\imath\left(g_{01}^{\prime}\right), \ldots, l\left(g_{d-1 d}^{\prime}\right)\right] & =\omega_{d}^{G}\left(g_{01}, \ldots, g_{d-1 d}\right) \\
& =\omega_{d}^{G^{\prime}}\left(g_{01}^{\prime}, \ldots, g_{d-1 d}^{\prime}\right)=\delta \beta_{d-1}^{G^{\prime}},
\end{aligned}
$$

thus split to lower $(d-1)$ dimensional $G^{\prime}$ cochains. Formally, we mean that a nontrivial $G$-cocycle

$$
\omega_{d}^{G} \in \mathcal{H}^{d}[G, U(1)]
$$

becomes a trivial element 1 (a coboundary) when it is pulled back (denoted as ${ }^{*}$ ) to $G^{\prime}$ :

$$
1=\iota^{*} \omega_{d}^{G} \in \mathcal{H}^{d}\left[G^{\prime}, U(1)\right] .
$$

The dimension of Hilbert space is restricted from a $|G|$ per degree of freedom in the bulk to a smaller $\left|G^{\prime}\right|$ per degree of freedom on the boundary.

As an application of Appendix E, we will count and classify distinct gauge-symmetry-breaking gapped interfaces in various dimensions (e.g., $2+1 \mathrm{D}$ bulk and $3+1 \mathrm{D}$ bulk), in Appendix F1.

\section{APPENDIX F: DYNAMICALLY GAUGED GAPPED INTERFACES OF TOPOLOGICALLY ORDERED GAUGE THEORIES}

Because gauge symmetry is not a physical symmetry but only a gauge redundancy, the physical meanings of gaugesymmetry breaking and gauge-symmetry extension are rather different from their global symmetry counterparts. We would like to reinterpret the dynamically gauged gapped interfaces for topologically ordered gauge theories (such that the whole systems are topologically ordered without any global symmetries) more carefully in any number of dimensions.

Let us propose the generic gauged gapped interfaces of topologically ordered gauge theories as follows. Let $L$ be the gauge group of a gauged interface; let $G_{\text {I }}$ and $G_{\text {II }}$ be the gauge groups of the left and right sector relative to the interface, respectively. Let $L$ be a group with a group homomorphism map to $G_{\mathrm{I}} \times G_{\mathrm{II}}$,

$$
L \rightarrow G_{\mathrm{I}} \times G_{\mathrm{II}},
$$

such that the product of the two cocycles of the two twisted gauge theories on the left and right pulls back to a trivial cocycle in $L$. Here, we assume neither a surjective map (as the gauge-symmetry extension) nor an injective map (as the gauge-symmetry breaking), but we only require the group homomorphism for $L \rightarrow G_{\mathrm{I}} \times G_{\mathrm{II}}$. Therefore, such a construction actually includes mixed mechanisms of gaugesymmetry extension and gauge-symmetry breaking, but we do not require any global symmetry at all. In Eq. (F1), we view $L$ and $G_{\mathrm{I}} \times G_{\mathrm{II}}$ all as gauge groups.

In Appendix F1, we explore applications of gaugesymmetry-breaking gapped interfaces. In Appendix F 2, we explore applications of gauge-symmetry-extended gapped interfaces, and we make a comparison to gapped interfaces obtained from first constructing global symmetry-extended SPTs and then dynamically gauging the system with various gauging procedures. The two subsections aim to demonstrate the generality of this Eq. (F1) for generic gauged interfaces.

\section{Gauge-symmetry-breaking gapped interface via} Anderson-Higgs mechanism-Examples: 2+1D twisted quantum double models $D^{\omega_{3}}(G)$ and $3+1 D$ gauge theories and Dijkgraaf-Witten gauge theories

The motivation for this subsection is to construct and count gauge-symmetry-breaking gapped interfaces for gauge theories, and to compare to the known methods and known examples in the past literature (mostly studied in the $2+1 \mathrm{D}$ bulk). Then, we can check consistency and further produce new concrete examples for gaugesymmetry-breaking gapped interfaces in any dimension. Many examples are shown in this appendix.

We consider Dijkgraaf-Witten (DW) gauge theories [22], namely, topologically ordered discrete $G$-gauge theories that allow "twists" by the cohomology group cocycle. For a more specialized case, a gauge-symmetry-breaking gapped boundary, this repeats the same setup in Eq. (E1) that we used in Appendix E. We only rewrite Eq. (F1) as $G^{\prime} \rightarrow$ $G \times 1$ with $L=G^{\prime}, G_{\mathrm{I}}=G$, and $G_{\mathrm{II}}=1$.

More generally, our strategy to construct and count distinct topological gapped interfaces between two given twisted gauge theories of $G_{\mathrm{I}}$ and $G_{\mathrm{II}}$ in any dimension, under Anderson-Higgs gauge-symmetry breaking, is [101]

(i) First step: For gauge-symmetry-breaking gapped interfaces, we consider Eq. (F1), with an additional constraint that $L \subseteq G_{\mathrm{I}} \times G_{\mathrm{II}}$ be an unbroken gauge subgroup. The criteria are (similar to Appendix E, except that every group is gauge group) that the $G_{\mathrm{I}} \times G_{\mathrm{II}}$-cocycle $\omega^{G_{\mathrm{I}} \times G_{\mathrm{II}}}=\omega_{\mathrm{I}}^{G_{\mathrm{I}}}\left(g_{\mathrm{I}}\right) \cdot \omega_{\text {II }}^{G_{\text {II }}}\left(g_{\mathrm{II}}\right)^{-1}(\mathrm{al}-$ lowed by the Künneth formula) in $\mathcal{H}^{d}\left[G_{\mathrm{I}} \times\right.$ $\left.G_{\text {II }}, U(1)\right]$ becomes a coboundary $1 \in \mathcal{H}^{d}[L, U(1)]$ when we restricted $G_{\text {I }}$ (on the left) and $G_{\text {II }}$ (on the right) to $L$ on the interface. 
(ii) Second step: To fully implement the first step, one has to actually pick a trivialization of the cocycle $\omega^{G_{I} \times G_{\text {III }}}$. The choice is not unique and we can modify it by appending any cocycle in $\mathcal{H}^{d-1}[L, U(1)]$, corresponding to a topological $L$-gauge theory on the boundary or interface, following Appendix D $2 b$. This yields distinct new gauged interfaces.

(iii) Third step: Some of the gauged interfaces, constructed by the above two steps, can be identified. For example, two different gauge groups $L_{1}$ and $L_{2}$ on the interfaces (between the same pair of bulk gauge groups) with cocycles $\omega_{d-1}^{L_{1}}$ and $\omega_{d-1}^{L_{2}}$ can be identified as the same gapped interface if and only if the two interfaces are conjugate through the adjoint action of $G_{\mathrm{I}} \times G_{\text {II }}$ [102]. Namely, some element $g \in G_{\mathrm{I}} \times G_{\mathrm{II}}$ identifies two interfaces by $g L_{1} g^{-1}=L_{2}$.

(iv) Fourth step: To construct and count all gaugesymmetry-breaking gapped interfaces, we consider all the possible subgroups $L \subseteq G_{\mathrm{I}} \times G_{\mathrm{II}}$, and all possible lower-dimensional distinct gauge theories in $\mathcal{H}^{d-1}[L, U(1)]$, and we identify the equivalence classes of them as in the third step.

Many examples of gauge interfaces are provided below in Appendix $\mathrm{F} 1$, including $2+1 \mathrm{D} G=Z_{2}$ gauge theory (namely, the $Z_{2}$ toric code and $Z_{2}$ topological order); $2+1 \mathrm{D} G=Z_{2}$ twisted gauge theory [namely, the $Z_{2}$ double semions, or $U(1)_{2} \times U(1)_{-2}$-fractional quantum Hall states]; and more generic 2+1D Dijkgraaf-Witten discrete gauge theories, also written as twisted quantum double models $D^{\omega_{3}}(G)$ of a gauge group $G$ with a twisted three-cocycle $\omega_{3}$ for $G=\left(Z_{2}\right)^{3}, D_{4}, Q_{8}$. We also consider $3+1$ D Dijkgraaf-Witten gauge theories of a gauge group $G$ with a twisted four-cocycle $\omega_{4}$.

We show that the gauge-symmetry-breaking mechanism reproduces the previous results on gapped boundaries or interfaces of $2+1 \mathrm{D}$ topological orders, either through the anyon condensation method or through the tunneling matrices constructed through modular $\mathcal{S}$ and $\mathcal{T}$ data, especially showing consistency with Ref. [84]. Furthermore, we can systematically obtain gapped interfaces in any dimension, such as in $3+1 \mathrm{D}$.

\section{a. Gauge-symmetry-breaking boundaries or interfaces of $Z_{2}$ toric code and $Z_{2}$ double-semion}

(1) Consider a $2+1 \mathrm{D} \quad G_{\mathrm{I}}=G=Z_{2}$ gauge theory (namely, the $Z_{2}$ toric code and $Z_{2}$ topological order) on the left, and $G_{\mathrm{II}}=1$ as a trivial vacuum on the right. The three-cocycle on the left is a trivial coboundary $\omega_{3}^{G}(g)=1$ and the cocycle on the right is also 1, but the Hilbert spaces of the left and right sides are different. We can consider either subgroups $L=G^{\prime}=1$ or $L=G^{\prime}=Z_{2}$, so that $G^{\prime} \rightarrow G$ provides a trivial cocycle when pulling back to $G^{\prime}$. The $G^{\prime}=1$ and $G^{\prime}=Z_{2}$ define the famous $e$-condensed or $m$-condensed gapped boundaries, achieved by Anderson-Higgs gauge-symmetry breaking. The two $e$ - and $m$-gapped boundaries have been constructed explicitly on the lattice Hamiltonian model [79] and have been realized field theoretically through strong coupling sine-Gordon interactions at boundaries [80]. Following Appendix E, given a bulk Abelian Chern-Simons action with a $K=\left(\begin{array}{l}0 \\ 20\end{array}\right)$ matrix for $Z_{2}$ gauge theory, the $e$ - or $m$-gapped boundaries are achieved by strong coupling interactions $\int d t d x g \cos \left(2 \Phi_{1}\right)$ and $\int d t d x g \cos \left(2 \Phi_{2}\right)$, on a Luttinger liquid boundary, respectively [80]. See Table VII for the details of these two gapped boundaries.

(2) Consider a $2+1 \mathrm{D} G=Z_{2}$ twisted gauge theory [namely, the $Z_{2}$ double semions, or $U(1)_{2} \times U(1)_{-2^{-}}$ fractional quantum Hall states] on the left, and $G^{\prime}=$ 1 as a trivial vacuum on the right. The three-cocycle on the left is nontrivial $\omega_{3}^{G}(g) \neq 1$, and the cocycle on the right is 1; again, the Hilbert spaces of the left and right sides are different. We can consider only the subgroups $G^{\prime}=1$, so that $G^{\prime} \rightarrow G$ provides a trivial cocycle when pulling back to $G^{\prime}$. The $G^{\prime}=1$ defines the semion-antisemion condensed gapped interface by Anderson-Higgs gauge-symmetry breaking. Following Appendix E, given a bulk Abelian ChernSimons action with a $K=\left(\begin{array}{cc}2 & 0 \\ 0 & -2\end{array}\right)$ matrix for a $Z_{2}$ twisted gauge theory, the gapped boundary is achieved by the strong coupling interaction $\int d t d x g \cos \left[2\left(\Phi_{1}+\Phi_{2}\right)\right]$, on a Luttinger liquid boundary [80]. Again, this unique gapped interface is also realized and consistent with earlier work [7982]. See Table VII for the data of a gapped boundary.

(3) Consider a $Z_{2}$ toric code on the left and a $Z_{2}$ doublesemion model on the right, as an example for the gauge-symmetry-breaking gapped interface. Equation (F1) becomes $L \rightarrow Z_{2} \times Z_{2}$ with a trivial coboundary $\omega_{3}^{G_{\mathrm{I}}}=1$ of $G_{\mathrm{I}}=Z_{2}$ on the left, and a nontrivial cocycle $\omega_{3}^{G_{\mathrm{II}}}$ of $G_{\mathrm{II}}=Z_{2}$ on the right, and gauge-symmetry breaking results in AndersonHiggs to $L=1$ or $L=Z_{2}$. This is consistent with two gapped interfaces between the $Z_{2}$ toric code and $Z_{2}$ double semions found in Ref. [84].

TABLE VII. Subgroup $G^{\prime}$ of a $Z_{2}, \mathcal{H}^{2}\left[G^{\prime}, U(1)\right]$, and gaugesymmetry-breaking boundaries in $2+1 \mathrm{D}$. Our result reproduces and agrees with the classification in Ref. [80]'s Table III and in Ref. [84]'s Appendixes I and II.

\begin{tabular}{lccc}
\hline \hline $\begin{array}{l}Z_{2}^{\prime} \text { 's } \\
\text { subgroup }\end{array}$ & $\begin{array}{c}Z_{2} \text { toric code } \\
\text { number of gauge } \\
G^{\prime}\end{array}$ & $\begin{array}{c}Z_{2} \text { double-semion } \\
\text { number of gauge } \\
\text { boundaries }\end{array}$ & $\begin{array}{c}\mathcal{H}^{2}\left[G^{\prime}, U(1)\right] \\
\text { boundaries }\end{array}$ \\
\hline$\{1\}=1$ & 0 & 1 & 1 \\
$Z_{2}$ & 0 & 1 & 0 \\
& 2 (total number) & 1 (total number) \\
\hline \hline
\end{tabular}




\section{b. Gauge-symmetry-breaking boundaries of $D\left(D_{4}\right)=D^{\omega_{3, I I I}}\left[\left(Z_{2}\right)^{3}\right]$}

Here, we consider a $2+1 \mathrm{D}$ twisted quantum double model $D^{\omega_{3, \text { III }}}\left[\left(Z_{2}\right)^{3}\right]=D\left(D_{4}\right)$. It can be described by a twisted Abelian gauge theory under a type III three-cocycle $\omega_{3, \text { III }}$ (see its definition in Ref. [28]) or a non-Abelian topological field theory action $\int\left[\left(\sum_{I=1}^{3}(2 / 2 \pi) B_{I} d A_{I}\right)+\right.$ $\left.\left(1 / \pi^{2}\right) A_{1} A_{2} A_{3}\right]$. Alternatively, we can regard it as a discrete $D_{4}$ gauge theory, with $D_{4}$ a dihedral group of order 8 . Now, we aim to count the distinct types of topological gapped boundaries based on gauge-symmetry breaking. Following Eqs. (E1) and (F1), we choose $G_{\mathrm{I}}=G=D_{4}$ and $G_{\mathrm{II}}=1$. What are the possible unbroken subgroups $L=G^{\prime}$ ? In Appendix D, Table VIII, we show the subgroup data for the $D_{4}$ group. Since $D\left(D_{4}\right)$ is an untwisted gauge theory with a trivial three-cocycle $1 \in \mathcal{H}^{3}\left[D_{4}, U(1)\right]$, when we pull 1 back from $D_{4}$ to any subgroup $G^{\prime} \subseteq D_{4}$, it is still a threecoboundary $1 \in \mathcal{H}^{3}\left[G^{\prime}, U(1)\right]$. Among the 10 subgroups of $D_{4}, 4$ of the $Z_{2}$ subgroups are identified to two sets of conjugate subgroups under the adjoint action [102]. For two $\left(Z_{2}\right)^{2}$ subgroups and one $D_{4}$, each of them offers two distinct gapped boundaries by appending lowerdimensional topological states due to $\mathcal{H}^{2}\left[G^{\prime}, U(1)\right]=$ $\mathbb{Z}_{2}$. Thus, the total distinct gauge-symmetry-breaking gapped interfaces have 11 types, which is consistent with topological gapped boundaries obtained from a different approach via modular $\mathcal{S}$ and $\mathcal{T}$ data in $2+1 \mathrm{D}$ [84]. See Table IX for the details of these 11 gapped boundaries.

\section{c. Gauge-symmetry-breaking boundaries of}

$$
\begin{gathered}
D\left(Q_{8}\right)=D^{\omega_{3, I I} \omega_{3, \mathrm{I}}}\left[\left(Z_{2}\right)^{3}\right] \text { in } 2+1 \mathrm{D} \text { and } Q_{8} \text { gauge } \\
\text { theory in 3+1D }
\end{gathered}
$$

Let us now consider gapped gauge interfaces of discrete quaternion $Q_{8}$ gauge theories in $2+1 \mathrm{D}$ and $3+1 \mathrm{D}$.

(1) First, we consider a $2+1 \mathrm{D}$ twisted quantum double model $D^{\omega_{3, \mathrm{II}} \omega_{3, \mathrm{I}}\left[\left(Z_{2}\right)^{3}\right]}=D\left(Q_{8}\right)$. It can be described by a twisted Abelian gauge theory under type III and

TABLE VIII. Subgroup $N$ and quotient groups $Q$ of $G=D_{4}$.

\begin{tabular}{lcc}
\hline \hline Subgroup $N$ & Quotient group $Q$ & $G / N=Q$ \\
\hline$\{1\}$ & $D_{4} /\{1\}=D_{4}$ & $D_{4} /\{1\}=D_{4}$ \\
$\left\{1, R^{2}\right\}$ (center) & $D_{4} /\left\{1, R^{2}\right\}=\left(Z_{2}\right)^{2}$ & $D_{4} / Z_{2}=\left(Z_{2}\right)^{2}$ \\
$\{1, x\}$ & No & No \\
$\left\{1, x R^{2}\right\}$ & No & No \\
$\{1, x R\}$ & No & No \\
$\left\{1, x R^{3}\right\}$ & No & No \\
$\left\{1, x, R^{2}, x R^{2}\right\}$ & $D_{4} /\left\{1, x, R^{2}, x R^{2}\right\}=Z_{2}$ & $D_{4} /\left(Z_{2}\right)^{2}=Z_{2}$ \\
$\left\{1, x R, R^{2}, x R^{3}\right\}$ & $D_{4} /\left\{1, x R, R^{2}, x R^{3}\right\}=Z_{2}$ & $D_{4} /\left(Z_{2}\right)^{2}=Z_{2}$ \\
$\left\{1, R, R^{2}, R^{3}\right\}$ & $D_{4} /\left\{1, R, R^{2}, R^{3}\right\}=Z_{2}$ & $D_{4} / Z_{4}=Z_{2}$ \\
$D_{4}$ & $D_{4} / D_{4}=1$ & $D_{4} / D_{4}=1$ \\
\hline \hline
\end{tabular}

TABLE IX. Subgroup $G^{\prime}$ of a dihedral $D_{4}, \mathcal{H}^{2}\left[G^{\prime}, U(1)\right]$, and gauge-symmetry-breaking boundaries in $2+1 \mathrm{D}$. Our result reproduces and agrees with the classification in Ref. [84]'s Appendix XI.

\begin{tabular}{lcc}
\hline \hline & & $\begin{array}{c}D\left(D_{4}\right)=D^{\omega_{3}, \mathrm{II}}\left[\left(Z_{2}\right)^{3}\right] \\
\text { number of distinct }\end{array}$ \\
$D_{4}$ 's subgroup $G^{\prime}$ & $\mathcal{H}^{2}\left[G^{\prime}, U(1)\right]$ & gauge boundaries \\
\hline$\{1\}=1$ & 0 & 1 \\
$\left\{1, R^{2}\right\}=Z_{2}$ & 0 & 1 \\
$\{1, \mathrm{x}\}=R\left\{1, \mathrm{x} R^{2}\right\} R^{-1}=Z_{2}$ & 0 & 1 \\
$\{1, \mathrm{x} R\}=R\left\{1, \mathrm{x} R^{3}\right\} R^{-1}=Z_{2}$ & 0 & 1 \\
$\left\{1, \mathrm{x}, R^{2}, \mathrm{x} R^{2}\right\}=\left(Z_{2}\right)^{2}$ & $\mathbb{Z}_{2}$ & 2 \\
$\left\{1, \mathrm{x} R, R^{2}, \mathrm{x} R^{3}\right\}=\left(Z_{2}\right)^{2}$ & $\mathbb{Z}_{2}$ & 2 \\
$\left\{1, R, R^{2}, R^{3}\right\}=Z_{4}$ & 0 & 1 \\
$D_{4}$ & $\mathbb{Z}_{2}$ & 2 \\
& & 11 (total number) \\
\hline \hline
\end{tabular}

type I three-cocycles $\omega_{3, \text { III }} \cdot \omega_{3, \mathrm{I}}$ (see its definition in Ref. [28]) or a non-Abelian topological field theory action $\int\left[\left(\sum_{I=1}^{3}(2 / 2 \pi) B_{I} d A_{I}\right)+\left(1 / \pi^{2}\right) A_{1} A_{2} A_{3}+\right.$ $\left.(1 / 2 \pi) A_{1} d A_{1}\right]$. Alternatively, we can regard it as a discrete $Q_{8}$ gauge theory, with $Q_{8}$ a quaternion group of order 8. Now, we count the distinct types of topological gapped boundaries based on gaugesymmetry breaking. Following Eqs. (E1) and (F1), we choose $G_{\mathrm{I}}=G=Q_{8}$ and $G_{\mathrm{II}}=1$. What are the possible unbroken subgroups $L=G^{\prime}$ ? In Appendix D, Table X, we show the subgroup data for the $Q_{8}$ group. When we pull $1 \in \mathcal{H}^{3}\left[Q_{8}, U(1)\right]$ for untwisted $D\left(Q_{8}\right)$ back from $Q_{8}$ to any subgroup $G^{\prime} \subseteq Q_{8}$, it is still a three-coboundary $1 \in \mathcal{H}^{3}\left[G^{\prime}, U(1)\right]$. Among the six subgroups of $Q_{8}$, none is identified under adjoint actions. None of them can append lower-dimensional topological states due to $\mathcal{H}^{2}\left[G^{\prime}, U(1)\right]=0$. Thus, the total distinct gauge-symmetry-breaking gapped interfaces have six types, which is consistent with topological gapped boundaries obtained from a different approach via modular $\mathcal{S}$ and $\mathcal{T}$ data in $2+1 \mathrm{D}$ [84]. See Table XI's fourth column for the details of these six gapped boundaries.

(2) Second, we consider a $3+1 \mathrm{D} Q_{8}$ gauge theory. For an untwisted gauge theory with a trivial fourcocycle $1 \in \mathcal{H}^{4}\left[Q_{8}, U(1)\right]$, when we pull 1 back

TABLE X. Subgroup $N$ and quotient groups $Q$ of $G=Q_{8}$.

\begin{tabular}{lcc}
\hline \hline Subgroup $N$ & Quotient group $Q$ & $G / N=Q$ \\
\hline$\{1\}$ & $Q_{8} /\{1\}=Q_{8}$ & $Q_{8} /\{1\}=Q_{8}$ \\
$\{1,-1\}$ (center) & $Q_{8} /\{1,-1\}=\left(Z_{2}\right)^{2}$ & $Q_{8} / Z_{2}=\left(Z_{2}\right)^{2}$ \\
$\{1, i,-1,-i\}$ & $Q_{8} /\{1, i,-1,-i\}=Z_{2}$ & $Q_{8} / Z_{4}=Z_{2}$ \\
$\{1, j,-1,-j\}$ & $Q_{8} /\{1, j,-1,-j\}=Z_{2}$ & $Q_{8} / Z_{4}=Z_{2}$ \\
$\{1, k,-1,-k\}$ & $Q_{8} /\{1, k,-1,-k\}=Z_{2}$ & $Q_{8} / Z_{4}=Z_{2}$ \\
$Q_{8}$ & $Q_{8} / Q_{8}=1$ & $Q_{8} / Q_{8}=1$ \\
\hline \hline
\end{tabular}


TABLE XI. Subgroup $G^{\prime}$ of a quaternion $Q_{8}, \mathcal{H}^{2}\left[G^{\prime}, U(1)\right]$, $\mathcal{H}^{3}\left[G^{\prime}, U(1)\right]$ and gauge-symmetry-breaking boundaries in $2+$ $1 \mathrm{D}$ and $3+1 \mathrm{D}$. Our $2+1 \mathrm{D}$ result reproduces and agrees with the classification in Ref. [84]'s Appendix XII. Our 3 + 1D result may be new to the literature.

\begin{tabular}{lccc}
\hline \hline & & & $\begin{array}{c}Q_{8} \text { gauge theories } \\
\text { number of distinct } \\
\text { gauge boundaries } \\
2+1 \mathrm{D} D\left(Q_{8}\right) \text { vs }\end{array}$ \\
$Q_{8}$ 's subgroup $G^{\prime}$ & {$\left[G^{\prime}, U(1)\right]\left[G^{\prime}, U(1)\right]$} & $3+1 \mathrm{D}$ \\
\hline$\{1\}=1$ & 0 & 0 & 1 vs 1 \\
$\{1,-1\}=Z_{2}$ & 0 & $\mathbb{Z}_{2}$ & 1 vs 2 \\
$\{1, i,-1,-i\}=Z_{4}$ & 0 & $\mathbb{Z}_{4}$ & 1 vs 4 \\
$\{1, j,-1,-j\}=Z_{4}$ & 0 & $\mathbb{Z}_{4}$ & 1 vs 4 \\
$\{1, k,-1,-k\}=Z_{4}$ & 0 & $\mathbb{Z}_{4}$ & 1 vs 4 \\
$Q_{8}$ & 0 & $\mathbb{Z}_{8}$ & 1 vs 8 \\
& & & 6 vs 23 (total number) \\
\hline \hline
\end{tabular}

from $Q_{8}$ to any subgroup $G^{\prime} \subseteq Q_{8}$, it is still a fourcoboundary $1 \in \mathcal{H}^{4}\left[G^{\prime}, U(1)\right]$. After appending lower-dimensional topological states (see Table XI's fourth column), we find 23 gapped boundaries.

\section{d. Gauge-symmetry-breaking boundaries of $G=Z_{2}$ or $\left(Z_{2}\right)^{2}$ twisted gauge theories in $3+1 D$}

Consider 3+1D Dijkgraaf-Witten gauge theories of a gauge group $G=Z_{2}$ and $\left(Z_{2}\right)^{2}$ with twisted four-cocycle $\omega_{4}$.

(1) First, we consider a $3+1 \mathrm{D} Z_{2}$ gauge theory, described by a low-energy $B F$ action $\int(2 / 2 \pi) B d A$ with two-form and one-form fields $B$ and $A$. Following Eqs. (E1) and (F1), we choose $G_{\mathrm{I}}=G=Z_{2}$ and $G_{\text {II }}=1$. What are the possible unbroken subgroups $L=G^{\prime}$ ? Since it is an untwisted gauge theory with a trivial four-cocycle $1 \in \mathcal{H}^{4}\left[Z_{2}, U(1)\right]$, when we pull 1 back from $Z_{2}$ to any subgroup $G^{\prime} \subseteq Z_{2}$, it is still a four-coboundary $1 \in \mathcal{H}^{4}\left[G^{\prime}, U(1)\right]$. There are two types of boundaries realized by condensing the $Z_{2}$ 's charge $e$-particle and condensing the $Z_{2}$ 's flux $m$-string on boundaries. These two boundaries are $e$ - and $m$-gapped boundaries, analogs to that of the $2+1 \mathrm{D} Z_{2}$ toric code. However, we can append a lower-dimensional topological state due to $\mathcal{H}^{3}\left[Z_{2}, U(1)\right]=\mathbb{Z}_{2}$; thus, we find three gapped boundaries, as shown in Table XII's third column.

(2) Second, we consider a $3+1 \mathrm{D}\left(Z_{2}\right)^{2}$ twisted gauge theory, described by a low-energy $B F$ action $\int\left(\sum_{I=1}^{2}(2 / 2 \pi) B_{I} d A_{I}\right)+\left(2 /(2 \pi)^{2}\right) A_{1} A_{2} d A_{2}$ with two-form and one-form fields $B$ and $A$. Following Eqs. (E1) and (F1), we choose $G_{\mathrm{I}}=G=\left(Z_{2}\right)^{2}$ and $G_{\mathrm{II}}=1$. What are the possible unbroken subgroups $L=G^{\prime}$ ? For a twisted gauge theory with a fourcocycle $\mathcal{H}^{4}\left[\left(Z_{2}\right)^{2}, U(1)\right]$, only limited subgroups $G^{\prime}$ trivialize the cocycle after pulling $G$ back to $G^{\prime}$. After appending lower-dimensional topological states, we find five gapped boundaries, as shown in Table XII's fourth column.

To summarize, in this section, we provide many gaugesymmetry-breaking gapped interfaces and detailed data. We find consistency with results obtained in previous literature (in $2+1 \mathrm{D}$ ), but we can systematically obtain gapped interfaces in any dimension, such as $3+1 \mathrm{D}$.

\section{Comparison to gapped interfaces obtained from dynamically gauging the symmetry-extended SPTs}

In Appendix D, we summarized how to construct symmetry-preserving gapped boundary for SPTs via Eq. (D1)'s symmetry extension $1 \rightarrow K \rightarrow H \stackrel{r}{\rightarrow} G \rightarrow 1$. In this section, we would like to explore various ways to dynamically gauge this SPT system to obtain different topologically ordered gauge versions of the system, and we make a comparison with the generic gauge interface construction in Eq. (F1)'s $L \rightarrow G_{\mathrm{I}} \times G_{\mathrm{II}}$. The goal is to demonstrate that the gauge interface construction from $L \rightarrow G_{\mathrm{I}} \times G_{\mathrm{II}}$ is general enough to contain different dynamical gauging procedures of the SPT system. To narrow down the possibilities of outcomes, here, we would like to fully gauge the left side SPTs of group $G$ to be a twisted gauge theory of group $G$ and to fully gauge the interface of group $H$. What remains are the different but consistent choices of gauging the right side of the interface. This corresponds to Eq. (F1), where we choose $G_{\mathrm{I}}=G$, $L=H$, and leave $G_{\text {II }}$ free for different choices. Below, we

TABLE XII. For $G=Z_{2}=Z_{2}^{(a)}$ or $G=\left(Z_{2}\right)^{2}=Z_{2}^{(a)} \times Z_{2}^{(b)}$, we list down the subgroup $G^{\prime}, \mathcal{H}^{2}\left[G^{\prime}, U(1)\right]$, and gauge-symmetry-breaking boundaries in $3+1 \mathrm{D}$.

\begin{tabular}{lccc}
\hline \hline$G^{\prime}$ s subgroup $G^{\prime}$ & $\mathcal{H}^{3}\left[G^{\prime}, U(1)\right]$ & $\begin{array}{c}3+1 \mathrm{D} G=Z_{2} \\
\text { gauge theory number } \\
\text { of gauge boundaries }\end{array}$ & $\begin{array}{c}3+1 \mathrm{D} G=\left(Z_{2}\right)^{2} \text { twisted } \\
\text { DW theorynumber of } \\
\text { gauge boundaries }\end{array}$ \\
\hline$\{1\}=1$ & 0 & 1 & 1 \\
$Z_{2}^{(a)}$ & $\mathbb{Z}_{2}$ & 2 & 2 \\
$Z_{2}^{(b)}$ & $\mathbb{Z}_{2}$ & & 2 \\
$\left(Z_{2}\right)^{2}$ & $\left(\mathbb{Z}_{2}\right)^{3}$ & & 0 \\
\hline \hline
\end{tabular}


provide several examples for the different choices of $G_{\text {II }}$ and interpret the construction from the perspectives of (a) gauging of the symmetry-extended SPTs and (b) the gauge interface of topologically ordered gauge theory systems, in a generic $d$-dimensional spacetime.

(1) Consider $H \rightarrow G \times 1$, where we choose $L=H$, $G_{\mathrm{I}}=G$, and $G_{\mathrm{II}}=1$ in Eq. (F1). The group homomorphism $H \rightarrow G \times 1$ is surjective, sending $h \in H$ to $[r(h), 1]=(g, 1) \in G \times 1$. From the gauging SPTs perspective of (a), the construction is obtained by first doing a local unitary transformation on the right sector to a trivial product state, which, thus, can be removed and regarded as a trivial vacuum. We only dynamically gauge the left sector $G$-SPTs and the $H$-interface to their gauge theory counterparts, namely, the $G$-twisted gauge theory (of Dijkgraaf-Witten) in $d$-dimensions and the $H$-gauge theory with a $G$-anomaly in a lower $(d-1)$ dimensions. But, we do not gauge the right sector and, thus, $G_{\mathrm{II}}=1$. From the gauge theory perspective of (b), the $H \rightarrow G \times 1$ construction means that we have a nontrivial inhomogeneous $G \times 1$-cocycle $\omega^{G \times 1}=\omega_{\mathrm{I}}^{G}(g) \cdot \omega_{\text {II }}^{1}(1)^{-1}=\omega_{\mathrm{I}}^{G}(g) \cdot 1$ for the gauge theory, and that can be pulled back to $H$ as lowerdimensional $H$-cochains to construct the interface gauge theory.

(2) Consider $H \rightarrow G \times G$, where we choose $L=H$, $G_{\mathrm{I}}=G$, and $G_{\mathrm{II}}=G$ in Eq. (F1). It is not surjective but only a group homomorphism from $h \in H$ to a diagonal group $[r(h), r(h)]=(g, g) \in G \times G$. From the gauging SPTs perspective of (a), the construction is obtained by first doing a local unitary transformation on the right sector to a trivial product state. The dynamically gauging procedure on the left sector and the interface is the same as in the previous case, but we also gauge the right sector to an untwisted usual $G_{\mathrm{II}}=G$-gauge theory. From the gauge theory perspective of (b), the $H \rightarrow G \times G$ construction means that we have a nontrivial inhomogeneous $G \times G$-cocycle $\omega^{G \times G}=\omega_{\mathrm{I}}^{G}(g) \cdot 1$ for the gauge theory with $\omega_{\text {II }}^{G}=1$, and that $\omega^{G \times G}$ can be pulled back to $H$ as lowerdimensional $H$-cochains to construct the interface gauge theory.

(3) Consider $H \rightarrow G \times H$, where we choose $L=H$, $G_{\mathrm{I}}=G$, and $G_{\mathrm{II}}=H$ in Eq. (F1). It is not surjective to $G \times H$, but it has a group homomorphism from $h \in H$ to $[r(h), h]=(g, h) \in G \times H$. From the gauging SPTs perspective of (a), the construction is obtained by first doing a local unitary transformation on the right sector to a trivial product state. The dynamically gauging procedure on the left sector and the interface is the same as in the previous case, but we also gauge the right sector to an untwisted usual $G_{\mathrm{II}}=H$-gauge theory. From the gauge theory perspective of (b), the $H \rightarrow G \times H$ construction means that we have a nontrivial inhomogeneous $G \times H$-cocycle $\omega^{G \times H}=\omega_{\mathrm{I}}^{G}(g) \cdot 1$ for the gauge theory with $\omega_{I I}^{H}=1$, and that $\omega^{G \times H}$ can be pulled back to $H$ as lower-dimensional $H$ cochains to construct the interface gauge theory.

More concretely, for a specific example, we can choose $G=Z_{2}$ and $H=Z_{4}$; from the perspective of gauging $2+$ 1D SPTs (a) from Eq. (D1), we choose $1 \rightarrow Z_{2}^{K} \rightarrow$ $Z_{4}^{H} \stackrel{r}{\rightarrow} Z_{2}^{G} \rightarrow 1$. The above constructions have the following implications. The first item above offers $Z_{4}^{H} \rightarrow Z_{2}^{G} \times 1$, which indicates that the left sector is a $2+1 \mathrm{D} Z_{2}$ doublesemion model (i.e., a twisted $Z_{2}$ gauge theory); the interface is a $1+1 \mathrm{D} Z_{4}$ gauge theory (with a $Z_{2}^{G}$ anomaly); and the right sector is a trivial vacuum (no gauge theory). The second item above offers $Z_{4}^{H} \rightarrow Z_{2}^{G} \times Z_{2}^{G}$, which indicates that the left sector is a $2+1 \mathrm{D} Z_{2}$ double-semion model; the interface is a $1+1 \mathrm{D} Z_{4}$ gauge theory (with a $Z_{2}^{G}$ anomaly); and the right sector is a $2+1 \mathrm{D} Z_{2}$ toric code (i.e., a $Z_{2}$ gauge theory). The second item above offers $Z_{4}^{H} \rightarrow Z_{2}^{G} \times Z_{4}^{H}$, which indicates that the left sector is a $2+1 \mathrm{D} Z_{2}$ double-semion model, the interface is a $1+1 \mathrm{D}$ $Z_{4}$ gauge theory (with a $Z_{2}^{G}$ anomaly), and the right sector is a $2+1 \mathrm{D} Z_{4}$ gauge theory.

The above construction requires a group homomorphism map, and we additionally need to impose the zero gauge flux constraint (more precisely, zero gauge holonomy for a shrinkable loop) everywhere, on the left sector, the interface, and the right sector. The previous three examples in Appendix F 2 all satisfy these constraints. However, other proposals may fail the constraints, for example, by considering $H \rightarrow G \times K$ for the gauge interface construction. This $H \rightarrow G \times K$ requests a construction of a $d$-dimensional $G$-twisted gauge theory on the left, a $(d-1)$-dimensional $H$ gauge theory (with $G$-anomaly) on the interface, and a $d$-dimensional untwisted usual $K$-gauge theory on the right. Will this be a valid construction? If we consider the $H \rightarrow G \times K$ map as $h \rightarrow[r(h), k]=(g, k)$, then it is not a group homomorphism, and the zero gauge flux constraint on the closed loop sitting between the interface (in $H$ ) and the right sector (in $K$ ) is generally nonzero. Thus, $H \rightarrow G \times K$ is illegal for a gauge interface construction between a $G$-twisted gauge theory and a $K$-gauge theory, at least from the perspective (a) of dynamically gauging global symmetry-extended SPTs.

However, we can make $H \rightarrow G \times K$ work for a gapped interface, if we consider it as a group homomorphism $H \times 1 \rightarrow G \times K$, so $(h, 1) \in H \times 1 \rightarrow[r(h), 1] \in G \times K$. This implies that we have a gauge-symmetry-extended construction from the left sector $H \rightarrow G$, but a gaugesymmetry-breaking construction from the right sector $1 \rightarrow K$. In short, the mixed symmetry-extension and 
symmetry-breaking construction can support an $H$-gauge interface between a $G$-twisted gauge theory on the left and an untwisted usual $K$-gauge theory on the right.

Overall, we show that the perspective (a) of gauging global symmetries of SPTs is within the construction of the perspective (b) of gauge interfaces of gauge theories based on Eq. (F1). This supports the generality of Eq. (F1).

[1] E. H. Lieb, T. D. Schultz, and D. C. Mattis, Two Soluble Models of an Antiferromagnetic Chain, Ann. Phys. (N.Y.) 16, 407 (1961).

[2] M. Karbach, K. Hu, and G. Müller, Introduction to the Bethe Ansatz II, Comput. Phys. 12, 565 (1998).

[3] F. D. M. Haldane, Continuum Dynamics of the 1-D Heisenberg Antiferromagnet: Identification with the O(3) Nonlinear Sigma Model, Phys. Lett. 93A, 464 (1983).

[4] I. Affleck, T. Kennedy, E. H. Lieb, and H. Tasaki, Valence Bond Ground States in Isotropic Quantum Antiferromagnets, Commun. Math. Phys. 115, 477 (1988).

[5] Z.-C. Gu and X.-G. Wen, Tensor-Entanglement-Filtering Renormalization Approach and Symmetry Protected Topological Order, Phys. Rev. B 80, 155131 (2009).

[6] M. Levin and C. P. Nave, Tensor Renormalization Group Approach to Two-Dimensional Classical Lattice Models, Phys. Rev. Lett. 99, 120601 (2007).

[7] X.-G. Wen, Topological Orders in Rigid States, Int. J. Mod. Phys. B 04, 239 (1990).

[8] X. Chen, Z.-C. Gu, and X.-G. Wen, Local Unitary Transformation, Long-Range Quantum Entanglement, Wave Function Renormalization, and Topological Order, Phys. Rev. B 82, 155138 (2010).

[9] X. Chen, Z.-C. Gu, and X.-G. Wen, Classification of Gapped Symmetric Phases in One-Dimensional Spin Systems, Phys. Rev. B 83, 035107 (2011).

[10] L. Fidkowski and A. Kitaev, Topological Phases of Fermions in One Dimension, Phys. Rev. B 83, 075103 (2011).

[11] N. Schuch, D. Perez-Garcia, and I. Cirac, Classifying Quantum Phases Using Matrix Product States and PEPS, Phys. Rev. B 84, 165139 (2011).

[12] F. Pollmann, E. Berg, A. M. Turner, and M. Oshikawa, Entanglement Spectrum of a Topological Phase in One Dimension, Phys. Rev. B 81, 064439 (2010).

[13] F. Pollmann, E. Berg, A. M. Turner, and M. Oshikawa, Symmetry Protection of Topological Phases in OneDimensional Quantum Spin Systems, Phys. Rev. B 85, 075125 (2012).

[14] X. Chen, Z.-X. Liu, and X.-G. Wen, Two-Dimensional Symmetry-Protected Topological Orders and Their Protected Gapless Edge Excitations, Phys. Rev. B 84, 235141 (2011).

[15] X. Chen, Z.-C. Gu, Z.-X. Liu, and X.-G. Wen, SymmetryProtected Topological Orders and the Cohomology Class of Their Symmetry Group, Phys. Rev. B 87, 155114 (2013).

[16] X. Chen, Z.-C. Gu, Z.-X. Liu, and X.-G. Wen, SymmetryProtected Topological Orders and the Cohomology Class of Their Symmetry Group, Science 338, 1604 (2012).
[17] X.-G. Wen, Construction of Bosonic Symmetry-ProtectedTrivial States and Their Topological Invariants via $g \times \operatorname{so}(\infty)$ Non-linear $\sigma$-Models, Phys. Rev. B 91, 205101 (2015).

[18] For $d+1 \mathrm{D}$ SPT states (possibly with a continuous symmetry), here we use the Borel group cohomology $\mathcal{H}^{d+1}[G, U(1)]$ or $\mathcal{H}^{d+1}[G \times S O(\infty), U(1)]$ to classify them $[14,17]$. Note that $\mathcal{H}^{d+1}[G, U(1)]=H^{d+2}(B G, \mathbb{Z})$, where $H^{d+2}(B G, \mathbb{Z})$ is the topological cohomology of the classifying space $B G$ of $G$. When $G$ is a finite group, we have only the torsion part $\mathcal{H}^{d+1}[G, U(1)]=$ $H^{d+2}(B G, \mathbb{Z})=H^{d+1}[B G, U(1)]$.

[19] A. Kapustin, Symmetry Protected Topological Phases, Anomalies, and Cobordisms: Beyond Group Cohomology, arXiv:1403.1467.

[20] A. Kapustin, Bosonic Topological Insulators and Paramagnets: A View from Cobordisms, arXiv:1404.6659.

[21] A. Kapustin, R. Thorngren, A. Turzillo, and Z. Wang, Fermionic Symmetry Protected Topological Phases and Cobordisms, J. High Energy Phys. 12 (2015) 052.

[22] R. Dijkgraaf and E. Witten, Topological Gauge Theories and Group Cohomology, Commun. Math. Phys. 129, 393 (1990).

[23] M. Levin and Z.-C. Gu, Braiding Statistics Approach to Symmetry-Protected Topological phases, Phys. Rev. B 86, 115109 (2012).

[24] L.-Y. Hung and X.-G. Wen, Quantized Topological Terms in Weakly Coupled Gauge Theories and Their Connection to Symmetry Protected Topological Phases, arXiv:1211.2767.

[25] S. Ryu, J. E. Moore, and A. W. W. Ludwig, Electromagnetic and Gravitational Responses and Anomalies in Topological Insulators and Superconductors, Phys. Rev. B 85, 045104 (2012).

[26] X.-G. Wen, Classifying Gauge Anomalies through SPT Orders and Classifying Gravitational Anomalies through Topological Orders, Phys. Rev. D 88, 045013 (2013).

[27] A. Kapustin and R. Thorngren, Anomalies of Discrete Symmetries in Various Dimensions and Group Cohomology, arXiv:1404.3230.

[28] J. C. Wang, Z.-C. Gu, and X.-G. Wen, Field Theory Representation of Gauge-Gravity Symmetry-Protected Topological Invariants, Group Cohomology and Beyond, Phys. Rev. Lett. 114, 031601 (2015).

[29] G.'t Hooft, Naturalness, Chiral Symmetry, and Spontaneous Chiral Symmetry Breaking, in Recent Developments in Gauge Theories, NATO Advanced Study Institutes Series (Series B. Physics), Vol. 59, edited by G.'t Hooft et al. (Springer, Boston, MA, 1980).

[30] The boundary theories of SPTs have anomalies [26-28]. The obstruction of gauging the global symmetries (on the SPT boundary) is known as the 't Hooft anomalies [29]. The possible boundary anomalies of SPTs include perturbative anomalies [31] and nonperturbative global anomalies [32,33]. Although SPTs can have both perturbative and nonperturbative anomalies, our construction of symmetric gapped interfaces is only applicable to SPTs with boundary nonperturbative anomalies.

[31] L. Alvarez-Gaume and E. Witten, Gravitational Anomalies, Nucl. Phys. B234, 269 (1984).

[32] E. Witten, An SU(2) Anomaly, Phys. Lett. 117B, 324 (1982). 
[33] E. Witten, Global Gravitational Anomalies, Commun. Math. Phys. 100, 197 (1985).

[34] We note that there is a terminology clash between condensed matter and high-energy/particle physics literature on the "Adler-Bell-Jackiw (ABJ) anomaly" [35,36]. In condensed matter literature [26], the phrase "ABJ anomaly" $[35,36]$ refers to "perturbative" anomalies (with $\mathbb{Z}$ classes, captured by the free part of cohomology/ cobordism groups), regardless of further distinctions (e.g., anomalies in dynamical gauge theory, global symmetry currents, etc.). In condensed matter terminology, the $\mathrm{ABJ}$ anomaly is captured by a one-loop diagram that only involves a fermion Green's function (with or without a dynamical gauge field). Thus, the one-loop diagram can be viewed as a property of a free fermion system even without gauge field. On the other hand, in high-energy/particle physics literature, the perturbative anomaly without a dynamical gauge field captured by a one-loop diagram should still be referred to as a perturbative 't Hooft anomaly, instead of the ABJ anomaly. Here, we attempt to use a neutral terminology to avoid any confusion.

[35] S. Adler, Axial-Vector Vertex in Spinor Electrodynamics, Phys. Rev. 177, 2426 (1969).

[36] J. Bell and R. Jackiw, A PCAC Puzzle: $\pi_{0} \rightarrow \gamma \gamma$ in the $\sigma$-Model, Nuovo Cimento A 60, 47 (1969).

[37] Here, we mean that there is no intrinsic $1+1 \mathrm{D}$ topological order in bosonic systems, neither in its own dimension nor on the boundary of any $2+1 \mathrm{D}$ bulk short-range entangled state. (Namely, we may say that there is no $1+1 \mathrm{D}$ bosonic topological quantum field theory robust against any local perturbation.) However, the $1+1 \mathrm{D}$ boundary of a $2+1 \mathrm{D}$ bulk long-range entangled state may have an intrinsic topological order. Moreover, in contrast, in a fermionic system, there is a $1+1 \mathrm{D}$ fermionic chain [38] with an intrinsic fermionic topological order.

[38] A. Y. Kitaev, Unpaired Majorana Fermions in Quantum Wires, Phys. Usp. 44, 131 (2001).

[39] A. Vishwanath and T. Senthil, Physics of Three Dimensional Bosonic Topological Insulators: Surface Deconfined Criticality and Quantized Magnetoelectric Effect, Phys. Rev. X 3, 011016 (2013).

[40] F. J. Burnell, X. Chen, L. Fidkowski, and A. Vishwanath, Exactly Soluble Model of a 3D Symmetry Protected Topological Phase of Bosons with Surface Topological Order, Phys. Rev. B 90, 245122 (2014).

[41] X. Chen, L. Fidkowski, and A. Vishwanath, Symmetry Enforced Non-Abelian Topological Order at the Surface of a Topological Insulator, Phys. Rev. B 89, 165132 (2014).

[42] C. Wang, A. C. Potter, and T. Senthil, Gapped Symmetry Preserving Surface State for the Electron Topological Insulator, Phys. Rev. B 88, 115137 (2013).

[43] M. A. Metlitski, C. L. Kane, and M. P. A. Fisher, A Symmetry-Respecting Topologically-Ordered Surface Phase of $3 d$ Electron Topological Insulators, Phys. Rev. B 92, 125111 (2015).

[44] P. Bonderson, C. Nayak, and X.-L. Qi, A Time-Reversal Invariant Topological Phase at the Surface of a $3 D$ Topological Insulator, J. Stat. Mech. (2013), P09016.

[45] M. A. Metlitski, L. Fidkowski, X. Chen, and A. Vishwanath, Interaction Effects on $3 D$ Topological Superconductors:
Surface Topological Order from Vortex Condensation, the 16 Fold Way and Fermionic Kramers Doublets, arXiv:1406.3032.

[46] D. F. Mross, A. Essin, and J. Alicea, Composite Dirac Liquids: Parent States for Symmetric Surface Topological Order, Phys. Rev. X 5, 011011 (2015).

[47] C. Wang, C.-H. Lin, and M. Levin, Bulk-Boundary Correspondence for Three-Dimensional SymmetryProtected Topological Phases, Phys. Rev. X 6, 021015 (2016).

[48] The symmetries may be ordinary unitary symmetries or may include antiunitary time-reversal symmetries.

[49] See Sec. III B for an example in which it is natural in condensed matter physics to treat $K$ as a global symmetry. See also a more recent work, Ref. [50], applying the idea to $1+1 \mathrm{D}$ bosonic/spin chains or fermionic chains.

[50] A. Prakash, J. Wang, and T.-C. Wei, Unwinding ShortRange Entanglement, arXiv:1804.11236.

[51] E. Witten, The "Parity" Anomaly on an Unorientable Manifold, Phys. Rev. B 94, 195150 (2016).

[52] N. Seiberg and E. Witten, Gapped Boundary Phases of Topological Insulators via Weak Coupling, Prog. Theor. Exp. Phys. 2016, 12C101 (2016).

[53] We remark that our approach to constructing gapped boundaries may not be applicable to some invertible topological orders (iTO, or the invertible topological quantum field theory) protected by no global symmetry. However, the gapped boundaries of certain iTO can still be constructed via our approach. For example, the $4+1 \mathrm{D}$ iTO with a topological invariant $(-1) \int w_{2} w_{3}$ has a boundary anomalous $3+1 \mathrm{D} Z_{2}$ gauge theory, which can be constructed by a suitable group extension via our Eq. (1.2). Here, $w_{i}=w_{i}(T M)$ is the $i$ th Stiefel-Whitney class of a tangent bundle $T M$ over spacetime $M$.

[54] M. Z. Hasan and C. L. Kane, Colloquium: Topological Insulators, Rev. Mod. Phys. 82, 3045 (2010).

[55] X.-L. Qi and S.-C. Zhang, Topological Insulators and Superconductors, Rev. Mod. Phys. 83, 1057 (2011).

[56] B. Bernevig and T. Hughes, Topological Insulators and Topological Superconductors (Princeton University Press, Princeton, NJ, 2013).

[57] L. Savary and L. Balents, Quantum Spin Liquids: A Review, Rep. Prog. Phys. 80, 016502 (2017).

[58] The name $C Z$ is read "controlled $Z$ " and is suggested by quantum computer science. The operator $U_{C Z, i j}$ measures $\sigma_{z}$ of spin $j$ if spin $i$ is in state $|\downarrow\rangle$ and otherwise does nothing.

[59] In the case of a compact ring boundary, $\left(\hat{U}_{Z_{2}}\right)^{2}=+1$ for an even-site boundary, while $\left(\hat{U}_{Z_{2}}\right)^{2}=-1$ for an odd-site boundary. To avoid the even or odd lattice site effect, from now on we assume the even-site boundary system throughout our work for simplicity. If there are no corners or spatial defects or curvature-which would lead to corrections in these statements - then the number of odd-site boundary components is always even, so, overall, $\hat{U}_{Z_{2}}^{2}=1$.

[60] It is not true that these states can be classified canonically by $\mathcal{H}^{d-1}[H, U(1)]$, because there is no natural starting point; that is, there is no natural choice of $\mu_{d-1}^{H}$ to begin 
with. Once one makes such a choice, the boundary states that we will construct can be classified by $\mathcal{H}^{d-1}[H, U(1)]$.

[61] In Sec. III B, we described a situation in which it is natural.

[62] The formula works when all groups are Abelian. For nonAbelian groups, there could be additional constraints on this formula, for example, in terms of conjugacy classes.

[63] To complete the argument, we need to know that, for every SPT phase with $G$-symmetry, a suitable extension $1 \rightarrow K \rightarrow H \stackrel{r}{\rightarrow} G \rightarrow 1$ exists. This is shown in Sec. V.

[64] Here, the local $G$-symmetry does not mean the gauge symmetry. On one hand, the local $G$-symmetry is that physically distinct configurations [note that, in the main text discussion, two distinct configurations are $\left(g_{i}, h_{i}, h_{i j}\right)$ and $\left.\left(g_{i}^{\prime}, h_{i}^{\prime}, h_{i j}^{\prime}\right)\right]$ have the same action amplitude. On the other hand, the gauge symmetry is not a (global) symmetry but, indeed, a gauge redundancy. The gauge symmetry is a gauge redundancy that two (redundant) configurations are, indeed, the same equivalent physical configuration and are related to each other through gauge transformations.

[65] Here, let us focus on the case that $K$ is Abelian (while $H$ and $G$ may be non-Abelian), for the simplicity of the formulas. One may generalize the situation to non-Abelian groups as well.

[66] L. Kong and X.-G. Wen, Braided Fusion Categories, Gravitational Anomalies, and the Mathematical Framework for Topological Orders in Any Dimensions, arXiv:1405.5858.

[67] Y. Tachikawa, On Gauging Finite Subgroups, arXiv: 1712.09542.

[68] After the appearance of our preprint on arXiv, one of the authors (J. W.) thanks Yuji Tachikawa for informing the recent Ref. [67]'s Sec. II. 7 as a mathematical proof, reproducing and obtaining similar results as our Sec. VA.

[69] X.-G. Wen, Quantum Orders and Symmetric Spin Liquids, Phys. Rev. B 65, 165113 (2002).

[70] Y. Hu, Y. Wan, and Y.-S. Wu, Twisted Quantum Double Model of Topological Phases in Two Dimensions, Phys. Rev. B 87, 125114 (2013).

[71] Y. Wan, J. C. Wang, and H. He, Twisted Gauge Theory Model of Topological Phases in Three Dimensions, Phys. Rev. B 92, 045101 (2015).

[72] A. Mesaros and Y. Ran, Classification of Symmetry Enriched Topological Phases with Exactly Solvable Models, Phys. Rev. B 87, 155115 (2013).

[73] Recall that the gauge transformation can be implemented on the basis (a vector in the Hilbert space) or on the wave function (effectively a "covector"). The operator $\hat{O}$ can be also implemented either on the basis as

$$
\hat{O}\left|\left\{n_{i j}\right\}_{M}\right\rangle=\sum_{\left\{n_{i j}^{\prime}\right\}} c_{\left\{n_{i j}^{\prime}\right\}}^{\left\{n_{i j}\right\}}\left|\left\{n_{i j}^{\prime}\right\}_{M}\right\rangle
$$

or on the wave function,

$$
\hat{O} \Phi\left(\left\{n_{i j}\right\}_{M}\right)=\sum_{\left\{\tilde{n}_{i j}\right\}} c_{\left\{n_{i j}\right\}}^{\left\{\tilde{n}_{i j}\right\}} \Phi\left(\left\{\tilde{n}_{i j}\right\}_{M}\right) .
$$

In either case, we obtain the same consistent result for $\hat{O}$ acting on the state vector $|\Phi\rangle$ as in Eq. (9.28).
[74] A. Y. Kitaev, Fault-Tolerant Quantum Computation by Anyons, Ann. Phys. (Amsterdam) 303, 2 (2003).

[75] To make a comparison, we remark that Refs. [27,76] show a related physics by starting from a given anomalous boundary topological field theory (TQFT) and finding the possible bulk TQFT.

[76] F. Benini, P.-S. Hsin, and N. Seiberg, Comments on Global Symmetries, Anomalies, and Duality in $(2+1) \mathrm{d}$, J. High Energy Phys. 4 (2017) 135.

[77] The free part of the cohomology/(co)bordism group contributes the perturbative anomalies. The torsion part of the cohomology/(co)bordism group contributes the nonperturbative global anomalies.

[78] A. Kapustin and N. Saulina, Topological Boundary Conditions in Abelian Chern-Simons Theory, Nucl. Phys. B845, 393 (2011).

[79] A. Kitaev and L. Kong, Models for Gapped Boundaries and Domain Walls, Commun. Math. Phys. 313, 351 (2012).

[80] J. Wang and X.-G. Wen, Boundary Degeneracy of Topological Order, Phys. Rev. B 91, 125124 (2015).

[81] M. Levin, Protected Edge Modes without Symmetry, Phys. Rev. X 3, 021009 (2013).

[82] M. Barkeshli, C.-M. Jian, and X.-L. Qi, Theory of Defects in Abelian Topological States, Phys. Rev. B 88, 235103 (2013).

[83] L.-Y. Hung and Y. Wan, Ground State Degeneracy of Topological Phases on Open Surfaces, Phys. Rev. Lett. 114, 076401 (2015).

[84] T. Lan, J. C. Wang, and X.-G. Wen, Gapped Domain Walls, Gapped Boundaries and Topological Degeneracy, Phys. Rev. Lett. 114, 076402 (2015).

[85] However, the fate of some of the gauge-symmetryextended interfaces turns out to be the same phase as the gauge-symmetry-breaking interface. This was later explored in Sec. 7 of Ref. [86], where their dual description and equivalence were found.

[86] J. Wang, K. Ohmori, P. Putrov, Y. Zheng, Z. Wan, M. Guo et al., Tunneling Topological Vacua via Extended Operators: (Spin-)TQFT Spectra and Boundary Deconfinement in Various Dimensions, Prog. Theor. Exp. Phys. (2018) 053A01.

[87] I. Cong, M. Cheng, and Z. Wang, Topological Quantum Computation with Gapped Boundaries, Prog. Theor. Exp. Phys. (2018) 053A01.

[88] K. G. Wilson, Confinement of Quarks, Phys. Rev. D 10, 2445 (1974).

[89] B. Zeng and X.-G. Wen, Gapped Quantum Liquids and Topological Order, Stochastic Local Transformations and Emergence of Unitarity, Phys. Rev. B 91, 125121 (2015).

[90] D. M. Greenberger, M. Horne, and A. Zeilinger, Bell's Theorem, Quantum Theory, and Conceptions of the Universe: Going beyond Bell's Theorem (Springer, Netherlands, 1989).

[91] Note that Sec. V's approach can only suggest the possible $K$ for a given $G$ and a given $G$-cocycle, but Sec. V cannot provide any analytic $H$-cochain easily.

[92] If $K$ is contained in the center of $H$, it implies $G$ acts trivially on $\mathcal{H}^{*}[K, U(1)]$. 
[93] Namely, the image of the $d_{2}$ map is guaranteed to be contained in the kernel of the inflation map from $\mathcal{H}^{d}[G, U(1)]$ to $\mathcal{H}^{d}[H, U(1)]$. J. W. gratefully acknowledges Tom Church and Ehud Meir for illuminating the spectral sequence method [94,95].

[94] K. S. Brown, Cohomology of Groups (Springer Science \& Business Media, New York, 2012), Vol. 87.

[95] https://mathoverflow.net/q/249368/27004 (Special thanks to Tom Church and E. Meir), " $g$ cocycle split and trivialized to a coboundary in $j$, given a group homomorphism $j \stackrel{r}{\rightarrow} g$," MathOverflow.

[96] J. Wang, L. H. Santos, and X.-G. Wen, Bosonic Anomalies, Induced Fractional Quantum Numbers and Degenerate Zero Modes: The Anomalous Edge Physics of SymmetryProtected Topological States, Phys. Rev. B 91, 195134 (2015).

[97] The $\mathcal{H}^{4}\left[Z_{2}^{T} \times S O(\infty), U_{T}(1)\right]=\left(\mathbb{Z}_{2}\right)^{2}$ classification [17] suggests a bulk topological invariant $\mathrm{e}^{\mathrm{i} 2 \pi \int \frac{1}{2} p_{1}}=(-1) \int p_{1}$, where the Pontryagin class $p_{1}$ is related by the Stiefel-Whitney class $w_{2}$ through the relation $w_{2}^{2}=p_{1}$ mod 2 on any closed oriented four-manifold. Moreover, the class with $w_{2}$ is related to $\pi_{1}[S O(\infty)]=\mathbb{Z}_{2}$ and $\pi_{1}[O(\infty)]=\mathbb{Z}_{2}$.

[98] This procedure has been shown explicitly in Ref. [50] recently.

[99] F. Haldane, Stability of Chiral Luttinger Liquids and Abelian Quantum Hall States, Phys. Rev. Lett. 74, 2090 (1995).

[100] Y.-M. Lu and A. Vishwanath, Theory and Classification of Interacting "Integer" Topological Phases in Two Dimensions: A Chern-Simons Approach, Phys. Rev. B 86, 125119 (2012).

[101] J. W. thanks Tian Lan for collaborating on a different approach in $2+1 \mathrm{D}$ [84].

[102] V. Ostrik, Module Categories, Weak Hopf Algebras and Modular Invariants, arXiv:math/0111139. 